\title{
Adiponectin mediates metabolic feedback to the mediobasal hypothalamic circadian clocks
}

\author{
Dissertation \\ for the award of the degree \\ "Doctor rerum naturalium" \\ Division of Mathematics and Natural Sciences \\ of the Georg-August-Universität Göttingen \\ submitted by \\ Anthony Hiu King Tsang \\ from Hong Kong SAR
}

Göttingen 2014 


\section{Thesis Committee}

Prof. Dr. Henrik Oster

Chronophysiology Group, Medical Department I, University of Lübeck, Lübeck, Germany

Prof. Dr. Thomas Pieler

Department of Developmental Biochemistry, University of Göttingen, Göttingen, Germany

Prof. Dr. Moritz Rossner

Molecular Neurobiology Group, Department of Psychiatry,

Ludwig-Maximilians-University, Munich, Germany

\section{Members of the Examination Board}

Referee: Prof. Dr. Henrik Oster

Chronophysiology Group, Medical Department I, University of Lübeck, Germany

$2^{\text {nd }}$ Referee: Prof. Dr. Thomas Pieler

Department of Developmental Biochemistry, University of Göttingen, Göttingen, Germany

\section{Further members of the Examination Board}

Prof. Dr. Moritz Rossner

Molecular Neurobiology Group, Department of Psychiatry,

Ludwig-Maximilians-University, Munich, Germany

Prof. Dr. Martin Göpfert

Dept. of Cellular Neurobiology, University of Göttingen, Göttingen, Germany

Dr. Henrik Bringmann

Dept. of Sleep and Waking, Max Planck Institute for Biophysical Chemistry, Göttingen, Germany

PD, Dr. Halyna Shcherbata

Gene expression and signaling Group, Max Planck Institute for Biophysical Chemistry, Göttingen, Germany

\section{Date of oral examination:}




\section{Declaration}

Herewith, I confirm that I have written the present PhD thesis independently and with no other sources and aids than quoted.

Göttingen, December 2014 


\section{Content}

Declaration 3

Contents $\quad 4$

Summary $\quad 6$

List of abbreviations $\quad 8$

1. Introduction 11

1.1. Mammalian circadian system 11

1.2. Interaction between metabolism and the circadian clock 13

1.3. Extra-SCN hypothalamic clocks in the mediobasal hypothalamus $\quad 15$

1.4. Objectives of the current study 17

2. Results 18

2.1. Establishment of the hypothalamic circadian reporter cell line 18

2.2. Adiponectin phase-resets molecular clocks in mediobasal hypothalamic neurons $\quad 21$

2.3. Characterization of the circadian phenotype of adiponectin deficient mice 25

2.4. The role of the molecular clock in $\mathrm{MBH}$ functions $\quad 27$

2.5. Adiponectin deficient mice have altered feeding rhythms 28

2.6. Tissue-specific effect of adiponectin on the circadian clocks in vivo 31

2.7. Npy is a direct target of the molecular clock 31

2.8. Adiponectin regulates food anticipatory circadian rhythms 33

2.9. Adiponectin upregulates Bmal1 expression in the mediobasal basal hypothalamic neurons $\quad 35$

2.10. Dose-dependency of adiponectin-induced phase resetting and Bmal1 induction 38

2.11. Tissue-specific effects of adiponectin on the circadian clocks in vitro 40

2.12. Differential roles of adiponectin receptors on in clock resetting 41

2.13. PGC1 $\alpha$ mediates the clock-modulating effect of adiponectin in MBH neurons 45

2.14. The role of central adiponectin signaling in regulating food anticipatory circadian rhythms 50

2.15. Role of adiponectin in the clock-modulating effects of high fat diet 55

3. Discussion $\quad 57$

3.1. Molecular mechanism of the circadian effects of adiponectin 58

3.2. The role of adiponectin in photic entrainment of circadian rhythms 59

3.3. The role of adiponectin in feeding rhythm regulation 60

3.4. The role of adiponectin in food anticipation regulation 61

3.5. The role of adiponectin in high fat diet induced circadian disturbances 63

3.6. Clinical implications $\quad 64$

3.7. Concluding remarks $\quad 65$

4. Materials and methods $\quad 67$

4.1. Animals and circadian behavioral experiments $\quad 67$

$\begin{array}{ll}\text { 4.2. Plasmid construction } & 67\end{array}$ 
4.3. Lentivirus production and transduction

4.4. Cell culture and circadian luminescence recording

4.5. Primary hypothalamic neuronal culture

4.6. ARC/ME slice cultures $\quad 70$

4.7. Quantitative real-time polymerase chain reaction 70

$\begin{array}{ll}\text { 4.8. Western blots } & 70\end{array}$

4.9. Chromatin immunoprecipitation $\quad 71$

4.10. NPY promoter end-point luciferase assays $\quad 72$

4.11. Intracerebroventricular administration $\quad 72$

4.12. ELISA analysis of plasma adiponectin $\quad 73$

4.13. Statistical analysis $\quad 73$

$\begin{array}{ll}\text { References } & 74\end{array}$

$\begin{array}{ll}\text { Curriculum vitae } & 84\end{array}$

$\begin{array}{ll}\text { Acknowledgement } & 90\end{array}$

Selected publications related to the current study 91

Interactions between endocrine and circadian systems $\quad 91$

Tissue-Specific Interaction of Per1-2 and Dec2 in the Regulation of Fibroblast Circadian Rhythms 107 


\section{Summary}

The rotation of the Earth around its axis results in a repetitive succession of day and night. The profound environmental changes associated with the day-night cycle drove most organisms to evolve endogenous timekeepers to reliably anticipate predictable events at particular times of day and adjust their behaviors and physiology accordingly. Such endogenous timekeeping machineries are known as circadian (from Latin circa diem - about a day) clocks. In mammals, the cellular time-keeping machinery is comprised of an interlocked transcriptional-translational feedback loop (TTL) that during the daytime the transcriptional activating BMAL1/CLOCK complexes activate their own repressors PERs and CRYs which will then be degraded during the night. This molecular clockwork regulates local cellular physiology and is shared among the central circadian pacemaker - the suprachiasmatic nucleus $(\mathrm{SCN})$ and other tissues in the brain and in the periphery. Recent studies have highlighted an extensive crosstalk between metabolism and circadian clock. For example, circadian misalignments contribute to metabolic disorders and vice versa. However, the mechanism of this link is still poorly understood.

The mediobasal hypothalamus (MBH) is an assembly of hypothalamic nuclei which together play a major role in regulating behavioral rhythms such as feeding/fasting and sleep/wake cycles. It has been documented that the autonomous cellular clockwork exists in multiple MBH nuclei and regulates the local physiology such as electrophysiological properties and appetite-regulating neuropeptides (NP) expression, hinting for the role of molecular clock in appetite regulation. One of the most important features of the MBH is its ability to integrate information carried by circulating metabolic hormones to regulate energy homeostasis of the body. I therefore hypothesize that there are metabolic hormones that can modulate the molecular clock in the $\mathrm{MBH}$ and thereby regulating feeding rhythms.

To search for metabolic hormones that can reset the MBH clock, I engineered a hypothalamic neuronal cell line to stably express a circadian reporter and used it as a model to screen for metabolic signals that are capable of resetting neuronal clocks. In a small scale screening, I identified an adipokines - adiponectin as a 
novel mediobasal hypothalamic cellular clock modulator. As it is known that circulating adiponectin levels are regulated by the metabolic status of the body, I further hypothesize that adiponectin is a mediator that can feed back to the MBH clocks according to the metabolic status of the body.

I demonstrated that adiponectin possesses a phase-resetting effect in multiple in vitro models of $\mathrm{MBH}$ neurons and an induction effects on Bmal1 transcription in $\mathrm{MBH}$ neurons both in vitro and in vivo. Further molecular analyses revealed that these circadian effects of adiponectin are, at least in part, mediated by a adiponectin receptor 1 (AdipoR1), peroxisome proliferator-activated receptor gamma coactivator 1 alpha (PGC1 $\alpha)$ and RAR-related orphan receptor alpha (ROR $\alpha)$ dependent mechanism.

Using adiponectin deficient (Adipoq KO) mice as a model, I investigated the role of adiponectin in circadian behavioral rhythms. Adipoq KO mice have largely normal circadian locomotor activity rhythms and photic entrainment of the circadian clock. However, they show significant dampened 24-hr feeding rhythms associated with altered diurnal profiles of clock and appetite-regulating gene expression in the MBH. Moreover, the mutants also show abnormal food entrainment of the locomotor activity under a time-restricted feeding (RF) regime - known as food anticipatory activity (FAA). Conversely, compared to ad libitum fed animals, the RF regime significantly enhances the circadian oscillation of plasma adiponectin, upregulates the diurnal expression of adiponectin receptors and Pgc1a clock genes associated with a profound reorganization of the diurnal expression patterns of appetite-regulating genes in the $\mathrm{MBH}$ of wild-type mice. Furthermore, central delivery of an antagonist of ROR $\alpha$ in wild-type mice could recapitulate the impaired FAA phenotypes of Adipoq KO mice. These data thus provide evidence to support the role of central adiponectin signaling in food entrainment of $\mathrm{MBH}$ clocks and feeding rhythms.

Together, these data reveal a novel metabolic feedback mechanism to the central circadian clocks. 


\section{List of abbreviations}

\begin{tabular}{|c|c|}
\hline 3'-UTR & 3' untranslated region \\
\hline aCSF & artificial cerebrospinal fluid \\
\hline AgRP & agouti-related peptide \\
\hline AMPK & adenosine monophosphate-activated protein kinase \\
\hline And & adiponectin \\
\hline ARC & arcuate nucleus \\
\hline BMAL1 & Brain and muscle Arnt-like protein-1 \\
\hline Bmal1-luc & Bmal1::LUCIFERASE \\
\hline CART & cocaine and amphetamine regulated transcript \\
\hline CCG & clock controlled genes \\
\hline CLOCK & Circadian Locomotor Output Cycles Kaput \\
\hline CNS & central nervous system \\
\hline CRY & Cryptochrome \\
\hline Ct & threshold cycle \\
\hline DBP & D site of albumin promoter (albumin D-box) binding protein \\
\hline DD & constant darkness \\
\hline Dex & dexamethasone \\
\hline DIV & day in vitro \\
\hline dKD & double knockdown \\
\hline DMH & dorsomedial nucleus of the hypothalamus \\
\hline DTT & dithiothreitol \\
\hline EDTA & ethylenediaminetetraacetic acid \\
\hline FAA & food anticipatory activity \\
\hline FAA & food anticipitatory activity \\
\hline fAdn & full-length adiponectin \\
\hline FEO & food entrainable oscillator \\
\hline Fors & forskolin \\
\hline gAdn & globular adiponectin \\
\hline GC & glucocorticoid \\
\hline GFP & green fluorescent protein \\
\hline HBSS & Hank's balanced salt solution \\
\hline HCRT & hypocretin \\
\hline HFD & high fat diet \\
\hline HMW & high molecular weight \\
\hline
\end{tabular}




\begin{tabular}{|c|c|}
\hline i.c.v. & Intracerebroventricular \\
\hline i.v. & intravenous \\
\hline IFU & infection unit \\
\hline IgG & immunoglobulin G \\
\hline IGL & intergeniculate leaflet \\
\hline ipRGC & intrinically photosensitive retinal ganglionic cells \\
\hline $\mathrm{KCl}$ & potassium chloride \\
\hline KD & knockdown \\
\hline КО & knockout \\
\hline LD & $12: 12$ hr light-dark \\
\hline LH & lateral hypothalamus \\
\hline MBH & mediobasal hypothalamus \\
\hline MC3R & melanocortin-3 receptor \\
\hline MEF & murine embryonic fibroblast \\
\hline mRNA & messenger ribonucleic acid \\
\hline $\mathrm{NaCl}_{2}$ & sodium chloride \\
\hline NAD & nicotinamide adenine dinucleotide \\
\hline $\mathrm{NaF}$ & sodium flouride \\
\hline $\mathrm{NaH} 2 \mathrm{PO} 4$ & sodium phosphate monobasic monohydrate \\
\hline $\mathrm{NaHCO} 3$ & sodium hydrogen carbonate \\
\hline $\mathrm{NaPP}_{\mathrm{i}}$ & sodium pyrophosphate \\
\hline $\mathrm{NaVO}_{5}$ & sodium orthovandate \\
\hline ND & normal diet \\
\hline neuropeptide & NP \\
\hline NPY & neuropeptide $Y$ \\
\hline ORF & open reading frame \\
\hline PER & Period \\
\hline Per2-luc & PER2::LUCIFERASE \\
\hline PFC & prefrontal cortex \\
\hline PGC1 $\alpha / \beta$ & peroxisome proliferator-activated receptor gamma coactivator $1 \mathrm{alpha/beta}$ \\
\hline PGK & phosphoglycerate kinase 1 \\
\hline POMC & pro-opiomelanocortin \\
\hline PPAR $\alpha$ & peroxisome proliferator-activated receptor alpha \\
\hline PPARY & peroxisome proliferator-activated receptor gamma \\
\hline PRC & phase response curve \\
\hline PVN & paraventricular nucleus of hypothalamus \\
\hline
\end{tabular}




\begin{tabular}{l|l} 
qPCR & Quantitative real-time polymerase chain reaction \\
\hline RHT & retinal-hypothalamic tract \\
\hline RORE & retinoic acid-related orphan receptor response element \\
\hline ROR $\boldsymbol{Q}$ & RAR-related orphan receptor alpha \\
\hline SCN & suprachiasmatic nucleus \\
\hline SDS-PAG & sodium dodecyl sulfate \\
\hline SDS-PAGE & SDS-Polyacrylamide gel electrophoresis \\
\hline SEM & standard error mean \\
\hline ShRNA & short hairpin ribonucleic acid \\
\hline SIRT & sirtuin \\
\hline TBS & Tris-buffer saline \\
\hline TNF $\boldsymbol{\alpha}$ & tumor necrosis factor alpha \\
\hline TTL & transcriptional-translational feedback loop \\
\hline VPLO & ventrolateral preoptic nucleus \\
\hline WT & wild-type \\
\hline $\boldsymbol{\alpha}-M S H$ & alpha-melanocyte-stimulating hormones \\
\hline
\end{tabular}




\section{Introduction}

In modern industrialized societies, there is an increasing prevalence of professions requiring irregular work hours. Extended shiftwork has been identified as a risk factor predisposing individuals to metabolic disorders $(1,2)$. Exposure to mistimed environmental time-cues (i.e., Zeitgebers) such as food intake leads to desynchrony among endogenous physiological rhythms and the external light/dark cycles and is believed to be one of the culprits that contributes to the adverse effects of shiftworking. Recent studies have highlighted an extensive crosstalk between metabolism and the circadian clock $(3,4)$. However, our understanding of the mechanistic links between these two systems is still largely incomplete.

\subsection{Mammalian circadian system}

The rotation of the Earth around its axis results in a repetitive succession of day and night. The profound environmental changes associated with the day-night cycle drove most organisms to evolve endogenous timekeepers to reliably anticipate predictable events at particular times of day and adjust their behaviors and physiology accordingly (5). Such endogenous timekeeping machineries are known as circadian (from Latin circa diem - about a day) clocks. In mammals, the cellular timekeeping machinery is comprised of a set of clock genes intertwined in a delayed interlocking transcriptional-translational feedback loop (TTL). Transcriptional activating Brain and muscle Arnt-like protein-1/ Circadian Locomotor Output Cycles Kaput (BMAL1/CLOCK) complexes activate the expression of their own repressors, Periods and Cryptochromes (Pers and Crys), during the day while high levels of PERs/CRYs protein accumulated in the nucleus during the night inhibit the activity of BMAL1/CLOCK and hence their own transcription. Progressive degradation of PERs and CRYs towards the end of the late night releases the inhibition and thus allows for the resumption of a new cycle of oscillation. This molecular clockwork regulates cellular physiology via controlling the rhythmic expression of E-box containing genes and is shared among the central circadian pacemaker - the suprachiasmatic nucleus (SCN) - and other tissues in the brain and in the periphery (Fig. 1). 


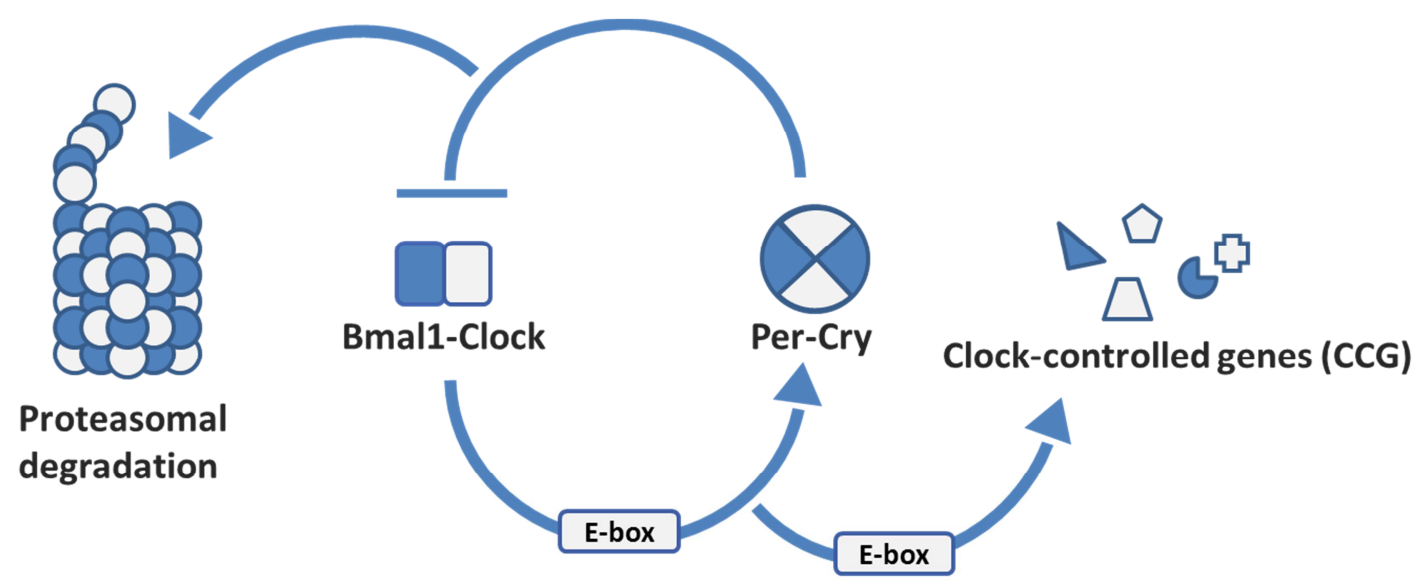

Transcriptional-translational feedback loop (TTL)

Figure 1. Molecular make-up of the mammalian cellular circadian clockwork

In mammals, the cellular timekeeping machinery is comprised of a set of clock genes intertwined with a delayed interlocking transcriptional-translational feedback loop (TTL). BMAL1/CLOCK complexes transcriptionally activates their own repressors Per1-3 and Cry1-2 via the E-box elements in their promoters during the day while the high level of PERs/CRYs accumulated in the nucleus during the night inhibits the activity of BMAL1/CLOCK and hence their own transcriptions. Progressive degradation of PERs and CRYs towards the end of the late night releases the inhibition and thus allows for the resumption of a new cycle of oscillation. This molecular clockwork regulates cellular physiology via controlling the rhythmic expression of other E-box containing clock control genes (CCGs).

In order to produce coherent physiological rhythms, the mammalian circadian system is organized in a hierarchical fashion in which the SCN synchronizes the circadian clocks of different physiological structures in both the periphery and the central nervous system (CNS) to the external light-dark cycle via multiple routes including direct neural connections, neuro-endocrine secretion and behavioral regulations (5) (Fig. 2). 


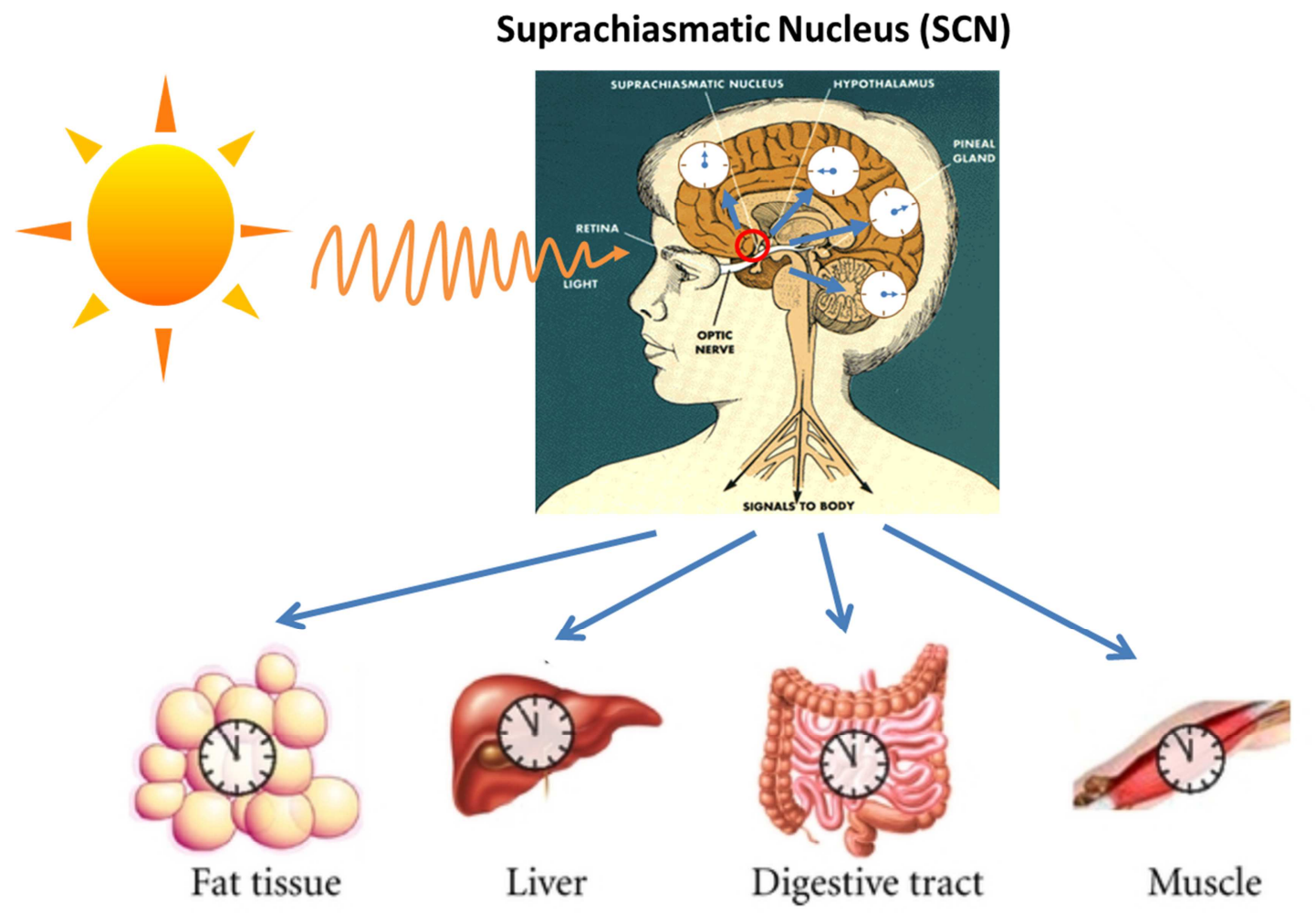

Figure 2. Schematic diagram of the hierarchical organization of mammalian circadian system. In order to generate coherent physiological and behavioral circadian rhythms, the local clocks residing in the periphery and the CNS are synchronized to the external day-night cycle by the SCN. The SCN clock is reset by the photic inputs transmitted from intrinsically photosensitive retinal ganglion cells (ipRGC) of the retina via the retinohypothalamic tract (RHT). The SCN clock then entrains the local clocks via multiple routes including direct neural connections, neuromodulators (in the CNS), hormones (in the periphery) and behavioral regulation

\subsection{Interaction between metabolism and the circadian clock}

Recently, metabolic signaling has been shown to impinge on the circadian system at various levels. Circadian clocks regulate feeding and metabolic rhythms of animals in sync with the environmental day/night cycle. Animals with mutations in clock genes are predisposed to develop feeding and metabolic deregulation $(6,7)$ (Table 1). Polymorphisms of clock genes have also been shown to associate with metabolic disorders in humans (8). Molecular clocks regulate a large array of metabolic pathways in various organs (5). Furthermore, many metabolic hormones such as ghrelin, glucocorticoids (GC), insulin and leptin are known to be regulated by the circadian clock on the one hand, and, on the other hand, are also known to feed back to the circadian system (9). Food is a potent Zeitgeber which can entrain and reset various non-SCN clocks 
while having little effect on the SCN. Restricted feeding during the normal rest phase can uncouple peripheral tissue clocks from the SCN (10). The exact food-regulated clock resetting mechanisms in different tissues are still not completely understood (7). However, at the molecular level a number of important metabolic sensors such as adenosine monophosphate-activated protein kinase (AMPK), cellular nicotinamide adenine dinucleotide redox state (i.e., $\mathrm{NAD}^{+} / \mathrm{NADH}$ ratio), peroxisome proliferator-activated receptor gamma coactivator 1 alpha (PGC1 $\alpha$ and $\beta$ ) and sirtuins (SIRT1 and 3 ) have been shown to impinge on molecular clock rhythms (7). However, the upstream physiological signals that couple to these metabolic pathways are still elusive. Together, not surprisingly many studies have demonstrated that compromised energy homeostasis of the body promotes circadian disruption (6). Feeding on diet that is rich in fat content (i.e., high fat diet; HFD) has been shown to disrupt circadian activity and local physiological rhythms even before the development of obesity (11). Moreover, mistimed feeding (i.e., eating during the normal rest phase) disturbs metabolic homeostasis $(12,13)$. When food access is temporally restricted to a particular time window of the day during the normal rest phase, animals will adapt by developing anticipatory behaviours and functions such as increased locomotor activity (i.e., food anticipatory activity; FAA), body temperature, glucocorticoid secretion just prior to the scheduled feeding. FAA rhythms represent a form of food entrainment of the circadian system as they persist even when the scheduled feeding condition is lifted (i.e., they free-run) (14). Food anticipation is believed to be advantageous for survival as it allows animals to organize their physiology for foraging in anticipation of a predictable food availability time window (15). Both the anatomical and molecular make-ups of the food entrainable oscillator (FEO) that is underlying FAA are still largely unknown. Of note, the SCN itself has been shown to suppress FAA $(15,16$ and unpublished data). Some controversy exists about whether canonical clock gene function is necessary for FAA (15, 17, 18). Both the CNS and periphery are involved in the development of FAA $(15,19)$. In the CNS, the hypothalamus, the reward circuitry and the cerebellum have been shown to regulate FAA rhythms while peripheral metabolic hormones are known to have modulatory roles (15). 
Table 1. Summary of the impacts of clock gene alterations on feeding and metabolic regulations

\begin{tabular}{|c|c|c|c|}
\hline Gene target & Metabolic/feeding phenotype & Food anticipatoty activity & Refs \\
\hline BMAL1 & Loss of feeding rhythm (arrhythmic) & Maintained & (88) \\
\hline CK1 & Altered activity, feeding, and metabolic rhythms in $\mathrm{CK}^{\text {tau }}$ & Maintained & (89) \\
\hline CLOCK $^{\Delta 19}$ & Hyperphagic, altered feeding rhythm, obese & Maintained & (76) \\
\hline CRY1/CRY2 & Loss of feeding rhythm (arrhythmic), hyperglycaemic & Maintained & $(90,91)$ \\
\hline Per1 & - & Maintained & (92) \\
\hline Per2 & Altered feeding rhythms, lean, altered lipid metabolism & Attenuated in Per2 $2^{\text {brdm }}$ & (92.93) \\
\hline Per1/2 & $\begin{array}{r}- \\
-\end{array}$ & $\begin{array}{l}\text { Maintained in Per } 1^{\text {ldc }} \text { Per } 2^{\text {Idc }} \\
\text { Attenuated in Per1 }{ }^{\%} / \text { Per2 }^{\text {brdm }}\end{array}$ & $(63,94)$ \\
\hline Per3 & Exacerbation of diet-induced obesity & - & (95) \\
\hline Reverb $\alpha$ & Obese, exacerbation of diet-induced obesity, altered lipid metabolism & - & (96) \\
\hline Reverb $\alpha / \beta$ & Arrhythmic, altered lipid metabolism & - & (63) \\
\hline RORa & Hyperphagic, lean, resistant to DIO & - & (97) \\
\hline
\end{tabular}

Adapted from Ref. 46

\subsection{Extra-SCN hypothalamic clocks in the mediobasal hypothalamus}

The mediobasal hypothalamus ( $\mathrm{MBH})$ is an assembly of hypothalamic nuclei, which controls body homeostasis and also plays a major role in regulating behavioral rhythms such as feeding/fasting and sleep/wake cycles (20). Despite the SCN has direct connections to multiple nuclei of the $\mathrm{MBH}$, it has also been documented that autonomous cellular clocks exist in the $\mathrm{MBH}$ that regulate local physiology such as electrophysiological properties and the expression of appetite-regulating neuropeptides (NPs) $(21,22)$ (Fig. 3). Diurnal expression rhythms of major NPs in the arcuate nucleus (ARC) - neuropeptide $\mathrm{Y}$ (NPY), agouti-related peptide (AgRP), pro-opiomelanocortin (POMC) and cocaine and amphetamine regulated transcript (CART) - have been documented (23-25). The importance of these appetite-regulating NPs in feeding rhythms was revealed in a number of studies using pharmacological and genetic approaches. For example, targeted ablation of NPY- or leptin- responsive neurons in the ARC profoundly disturbs diurnal feeding rhythms $(26,27)$. Genetic deletion of NPY receptors also results in altered feeding rhythms $(28)$. More recently, postnatal ablation of AgRP expressing neurons has been shown to impair food anticipation (29). Along with these observations, neurons in the lateral hypothalamus (LH) expressing the orexigenic neuropeptide hypocretin (HCRT or also called orexin), which are downstream to the ARC's NPY neurons, are also activated during food anticipation (30). Anorexinergic NPs appear to play a less important role in feeding rhythms. POMC is a precursor of multiple neuropeptides including the anorexigenic 
alpha-melanocyte-stimulating hormones ( $\alpha$-MSH) which exerts its effects via melanocortin receptors (24). POMC deficient mice have shown to retain largely normal circadian activity and feeding rhythms, albeit showing altered nocturnal meal-patterns (31). On the other hand, the loss of melanocortin-3 receptor (MC3R) has been shown to significantly impair food anticipation under restricted feeding conditions (32). The dorsomedial nucleus of the hypothalamus $(\mathrm{DMH})$ is an important integrating center that relays circadian inputs from the SCN to other physiological systems due to its direct connection to various brain nuclei such as the ARC, the LH, ventrolateral preoptic (VLPO) nuclei that regulate sleep/wake cycle, and the paraventricular nucleus of hypothalamus (PVN) controlling glucocorticoid secretion (33). Lesioning the DMH significantly disrupts circadian behavioral rhythms (34). The DMH has also been suggested as an important brain structure controlling FAA (35), but also (36).
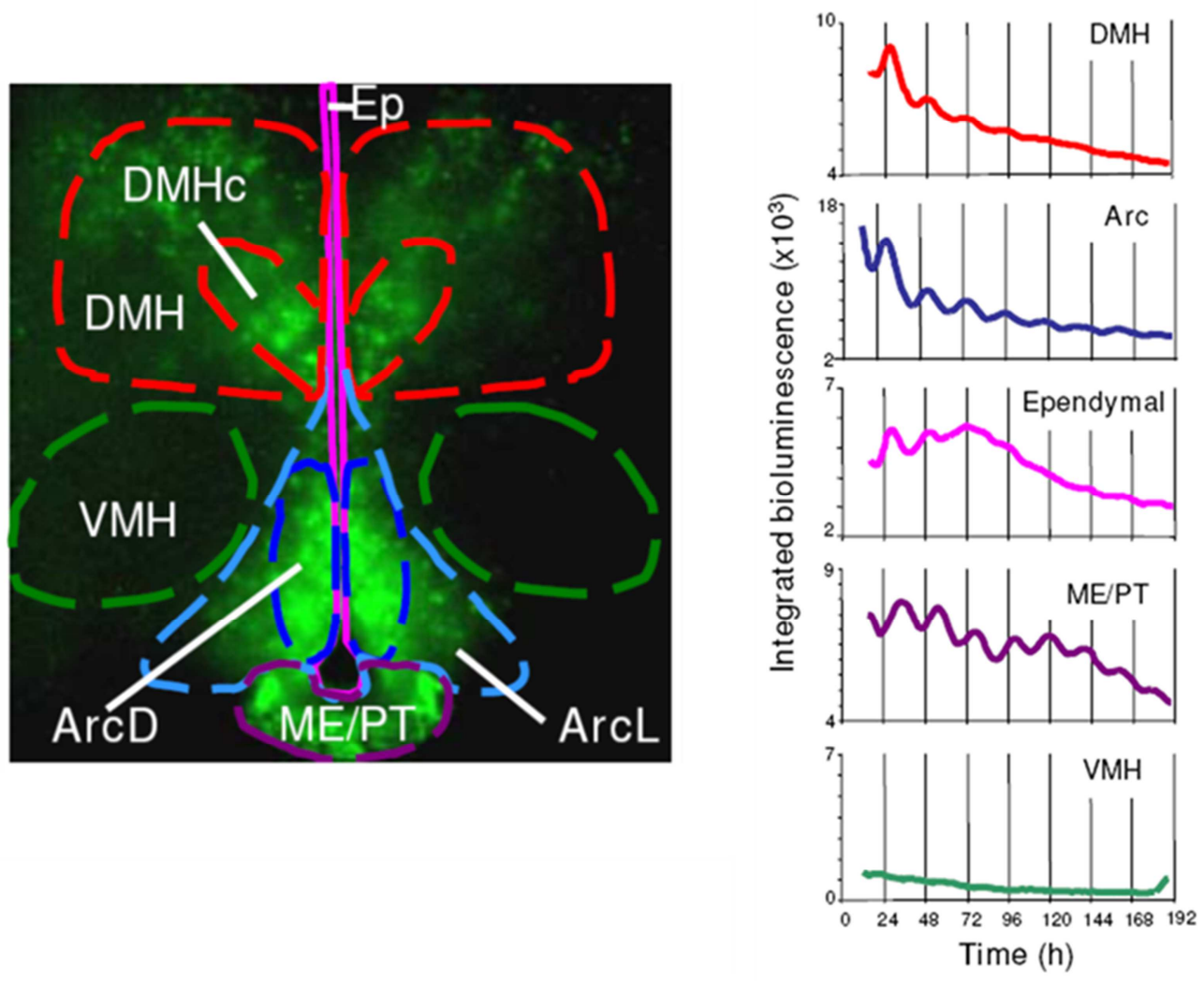

Figure 3. Self-sustaining molecular rhythms in various nuclei of the mediobasal hypothalamus organotypic slice cultures from Per2-luc mice. DMH: Dorsomedial hypothalamic nuceli, DMHc: DMH core, EP: Ependymal lining, ArcD: dorsal arcuate nucleus, ArcL: lateral arcuate nucleus, ME/PT: median eminence/pituitary, VMH: ventromedial hypothalamic nuclei. Adapted from Ref. 21. 


\subsection{Objectives of the current study}

One of the most important features of the MBH's nuclei is the ability to perceive and integrate metabolic information carried by peripheral hormones to stabilize energy homeostasis of the body. Feeding-related hormones such as insulin, ghrelin and leptin have been shown to regulate appetite and energy expenditure via the MBH (20). Moreover, leptin and ghrelin have also been shown to regulate feeding rhythms and FAA (19). However, the physiological and molecular mechanisms conferring these effects are still elusive. I therefore hypothesize that metabolic hormones can reset molecular clocks in the $\mathrm{MBH}$ and thereby regulate feeding rhythms. To identify such factors, I established a cell-based approach to screen a list of candidate metabolic hormones for their ability to reset the cellular clock of hypothalamic neuronal cells. I identified an adipokines - adiponectin - as a novel mediobasal hypothalamic cellular clock modulator. In the periphery, adiponectin is known to enhance insulin sensitivity, modulating fatty acid oxidation and suppressing inflammation (37). In the CNS, it is involved in regulating food intake, energy expenditure and mood $(38,39)$. Importantly, circulating adiponectin levels are regulated by the metabolic status of the body. In a situation of energy excess (such as obesity) adiponectin secretion is suppressed. In contrast, during fasting, both circulating adiponectin level and the expression of its cognate receptors in the MBH are upregulated (40). Thus, I further hypothesize that adiponectin is a novel MBH circadian clock modulator which can modify the circadian feeding behaviour. In the current study, I employ in vitro models of $\mathrm{MBH}$ neurons as well as adiponectin deficient mice as an in vivo model to investigate the potential role of adiponectin as a modifier of the mediobasal hypothalamic circadian clock. 


\section{Results}

\subsection{Establishment of the hypothalamic circadian reporter cell line}

To search for metabolic hormones capable of resetting the MBH cellular clock, I used an established SV40-immortalized mHypoE-N44 (N44 hereafter) cell line of embryonic hypothalamic neuronal origin (22). This cell line has been characterized to be GABAergic and express both Npy and Agrp, but not Cart and Pomc (22). In addition, the circadian oscillation of the molecular clockwork has been demonstrated in this cell line (22). To monitor the cellular rhythm in real-time, I engineered N44 cells to stably express a circadian reporter - Bmal1::LUCIFERASE (Bmal1-luc) in which the expression of luciferase is under the control of the Bmal1 promoter - via lentiviral transduction (41). After synchronization with dexamethasone (Dex), Bmal1-Luc activity displayed a self-sustained circadian oscillation with a period length of $23.67 \pm 0.1 \mathrm{hr}$ (means $\pm \mathrm{SEM}$, $\mathrm{n}$ = 21) when cultured in serum-free, B-27 supplemented medium (Fig. 4 A). With this reporter cell line, I then set up a screening paradigm in which different peptide hormones were applied to the cells at roughly two opposite phases of the first circadian cycle (hr 13 and 23) separately and the peak times of the two subsequent circadian cycles after treatment were used as readout (Fig. 4 A). This anti-phasic treatment scheme was used to circumvent the potential existence of circadian dead zones, i.e. phase intervals at which the clock would be non-responsive to an otherwise active agent. I validated this experimental setting with two well established positive controls of clock-resetting reagents, forskolin (Fors; an adenylate cyclase activator) and Dex (a glucocorticoid receptor agonist) $(5,42)$. As expected, both of them significantly reset the cellular clock of N44 cells in a phase-dependent manner (Fig. 4 B - D). I then selected a short list of metabolic peptide hormones based on their reported binding sites and physiological effects on the $\mathrm{MBH}$ from the literature for our screen (Table 2). Among all candidates, only (globular) adiponectin (gAdn) showed significant effects on resetting the phase of the cellular circadian rhythm (Fig. 4 F \& G). As a representative of the negative candidates, luminescence recordings from N44 Bmal1-luc cells treated with leptin are shown (Fig. 4 E). Adiponectin is an adipokine that has been shown to modulate a wide range of physiological systems in, both, the periphery and the CNS. Importantly, its functions are regulated by the metabolic state of the body (40). These screen data suggested that adiponectin may be a novel link between peripheral 
energy metabolism and circadian clocks in the CNS. Thus I decided to further study the role of adiponectin in circadian rhythm regulation in detail.

A

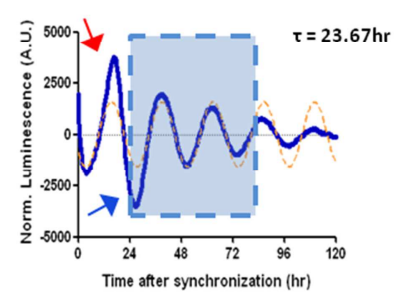

D

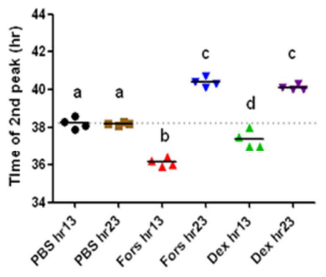

G

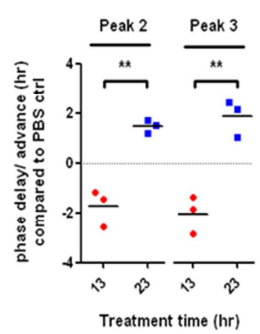

B

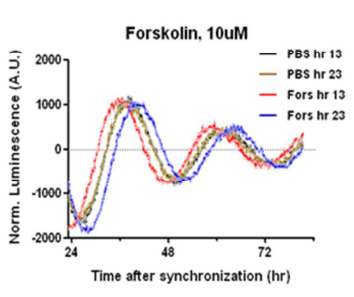

$\mathrm{E}$

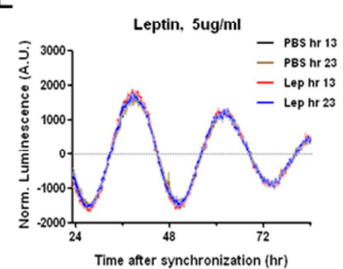

$\mathrm{H}$

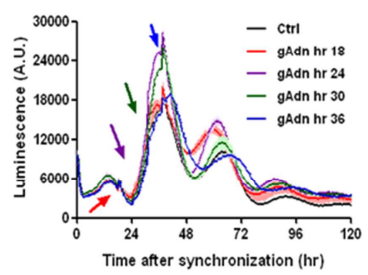

C

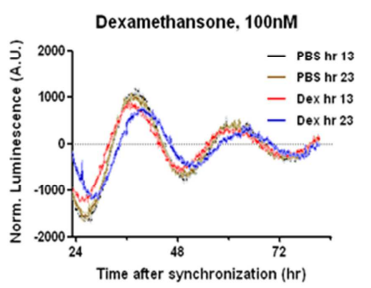

$\mathrm{F}$

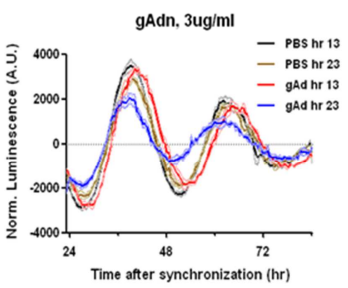

।

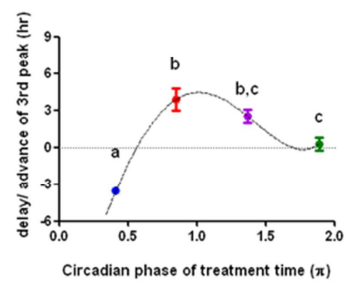

\section{Figure 4. Adiponectin resets molecular clock in N44 cells}

(A) A representative normalized bioluminescence recording of N44 Bmal1-luc cells synchronized by $2 \mathrm{hr}$ dexamethasone shock. The period length was calculated from 21 independent recordings across 5 passages. Two arrows (at hr13 and hr23 after synchronization) indicate the time points for the drug treatments in the peptide hormone screen. The peak times of the 2 nd and 3 rd circadian cycle were chosen as the readout of the screen.

(B - D) Normalized luminescence recordings from synchronized N44 Bmal1-Luc cells treated with forskolin (B) and dexamethansone (C) as a positive control of the screen, respectively. (D) Analysis of the peak time of the 2 nd circadian cycle of Bmal1-luc ( $n=4)$.

(E) Leptin $(5 \mathrm{ug} / \mathrm{ml})$ as a representative negative candidate of the screen $(n=4)$.

(F and G) gAdn as a positive candidate of the screen. Normalized luminescence recordings (F) and peak time analysis (G) of N44 Bmal1-Luc cells treated with gAdn (3ug/ml; n=3).

( $H$ and I) Phase-dependent response of gAdn-induced phase-shift in N44 Bmal1-Luc cells. (H) gAdn (5ug/ml) was treated on cells at indicated time points after synchronization. The peak time of the 3rd circadian cycle were used as readout. 
(I) Phase-dependent response analysis of $(\mathrm{H})$. The treatment time was adjusted into radian of the circadian Bmal1-luc rhythms. Colour code for the treatment time is consistent with (I) ( $n=3$ per time point).

Error bars indicate means \pm SEM. ${ }^{* *} p<0.01, * * * p<0.001$, Student's t-test. Groups denoted with different alphabets indicates statistical significance $(p<0.05)$, One-Way ANOVA with Tukey post-test.

Table 2. Summary of the result from the screen for MBH clock-modulating metabolic peptide hormones

\begin{tabular}{|c|c|c|}
\hline Drugs & Treatment 1 (at hr 13) (hr) & $\begin{array}{c}\text { Treatment } 2 \text { (at hr 23) } \\
\text { (hr) }\end{array}$ \\
\hline Forskolin, 10uM & $+2.21 \pm 0.11 * * *$ & $-2.18 \pm 0.13^{* * *}$ \\
\hline $\begin{array}{c}\text { Dexamethansone, } \\
\text { 100nM }\end{array}$ & $+0.95 \pm 0.24 *$ & $-1.90 \pm 0.07 * * *$ \\
\hline Apelin 13, 3ug/ml & $-0.96 \pm 0.52$ & $-0.37 \pm 0.38$ \\
\hline gAdn, 3ug/ml & $-1.74 \pm 0.43 * *$ & $+1.47 \pm 0.15^{*}$ \\
\hline TNFa, 50ng/ml & $-0.44 \pm 0.13$ & $-0.17 \pm 0.19$ \\
\hline IL6, 50ng/ml & $-0.50 \pm 0.38$ & $-0.77 \pm 0.30$ \\
\hline IGF1, 100ng/mI & $+0.00 \pm 0.18$ & $-0.67 \pm 0.06$ \\
\hline Chemerin 9, 2nM & $-0.80 \pm 0.27$ & $-0.34 \pm 0.34$ \\
\hline Chemerin 9, 1uM & $+0.16 \pm 0.39$ & $-0.50 \pm 0.32$ \\
\hline Chemerin $15,2 \mathrm{nM}$ & $-0.54 \pm 0.15$ & $-0.30 \pm 0.15$ \\
\hline Chemerin 15, 1uM & $-0.10 \pm 0.18$ & $-0.37 \pm 0.32$ \\
\hline Oxyntomodulin, 0.5 uM & $+0.43 \pm 0.12$ & $-0.07 \pm 0.23$ \\
\hline Leptin, 5ug/ml & $+0.15 \pm 0.14$ & $+0.21 \pm 0.15$ \\
\hline Insulin, 10ug/ml & $-0.10 \pm 0.06$ & $0.23 \pm 0.14$ \\
\hline Nesfatin, 3ug/ml & $-0.10 \pm 0.22$ & $-0.34 \pm 0.13$ \\
\hline Visfatin, 3ug/ml & $-0.07 \pm 0.14$ & $+0.03 \pm 0.16$ \\
\hline Ghrelin, 100nM & $+0.00 \pm 0.16$ & $+0.07 \pm 0.06$ \\
\hline Glucagon, 100nM & $-0.40 \pm 0.20$ & $-0.27 \pm 0.17$ \\
\hline Resistin, 10ng/ml & $-0.25 \pm 0.14$ & $-0.05 \pm 0.05$ \\
\hline GLP-1, 100nM & $-0.30 \pm 0.13$ & $+0.23 \pm 0.17$ \\
\hline
\end{tabular}

+ve: phase advance; -ve: phase delay; \pm SEM, ${ }^{*} \mathrm{p}<0.05,{ }^{* *} \mathrm{p}<0.01,{ }^{* * *}<0.001$ vs PBS ctrl; $\mathrm{N}=3-4$ 


\subsection{Adiponectin phase-resets molecular clocks in mediobasal hypothalamic neurons}

From the screen, I had observed that adiponectin can phase-reset the Bmal1-luc rhythm in a phase-dependent manner with opposite directions as observed after forskolin or dexamethasone treatments (Fig. 4 D \& G), suggesting the existence of a distinct resetting mechanism. To further characterize and confirm the phase-dependency of adiponectin's clock-resetting effect, synchronized N44 Bmal1-luc cells were treated around the course of the first circadian cycle at 6-hr intervals with constant real-time luminescence recording to allow for a more precise determination of the circadian phase at the time of treatment. Using the peak time of the subsequent circadian cycle as readout allowed me to construct a phase response curve (PRC), which confirmed the phase-dependent clock resetting effect of gAdn on hypothalamic cells (Fig. $4 \mathrm{H}-$ I) distinct from the PRCs of Fors and Dex (Fig. 5). 


\section{Forskolin}

A
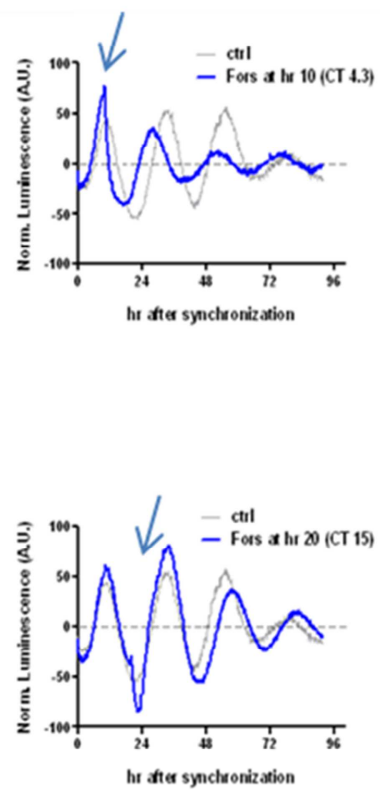

C

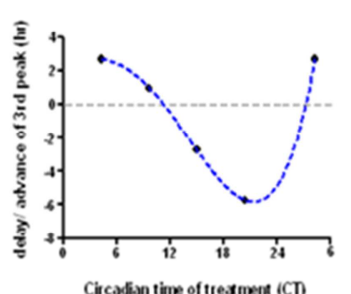

\section{Dexamethasone}

B
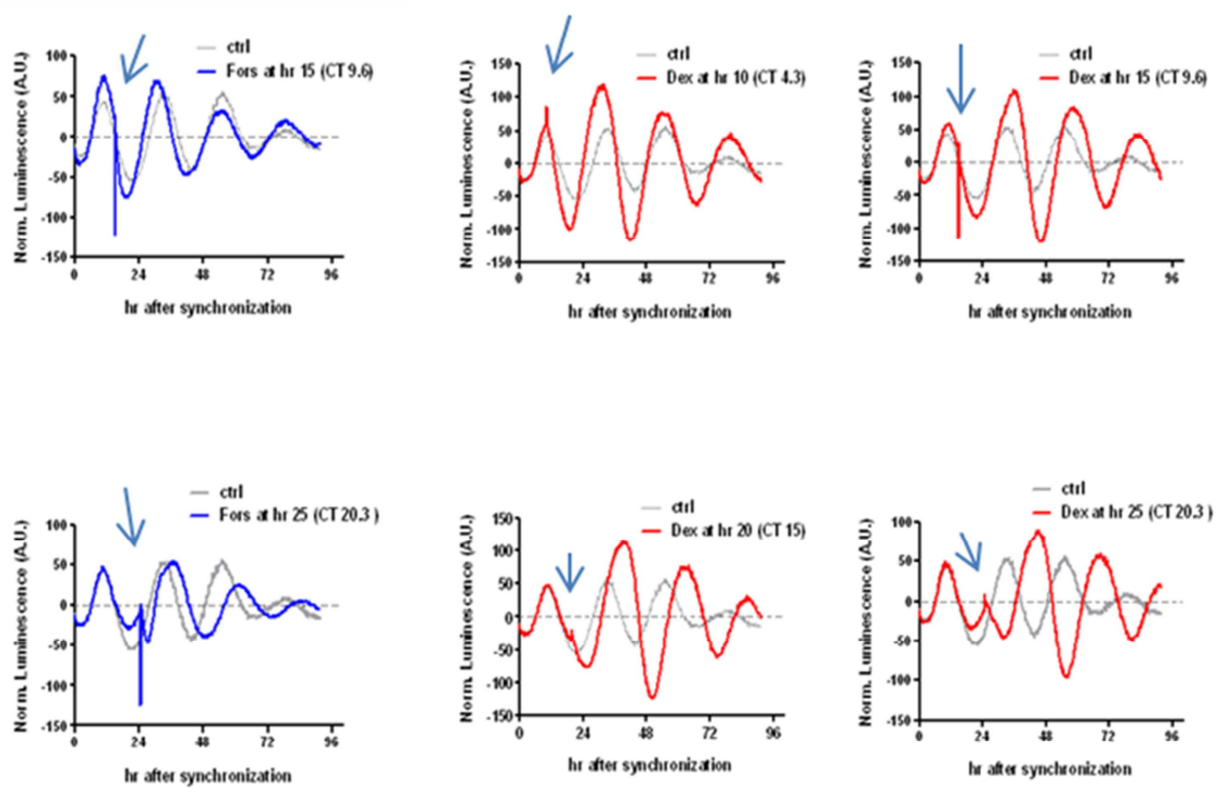

D

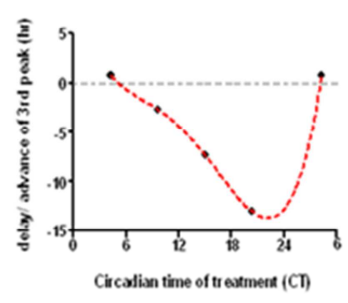

Figure 5. Resetting of molecular clock in N44 cells by forskolin and dexamethasone

(A and B) Representative normalized bioluminescence recordings of synchronized N44 Bmal1-luc cells treated with 10uM forskolin (A) and 100nM dexamethasone (B) at indicated time points. Grey curves are PBS treated controls.

(C and D) Phase-dependent response of Fors-induced (C) and Dex-induced (D) phase-shift of Bmal1-luc rhythms in N44 Bmal1-luc cells. Peak time of the $3^{\text {rd }}$ circadian cycles was used as readout. Treatment time was adjusted into circadian time of Bmal1-luc rhythm with СТ0 defined as the middle point of the ascending phase from the trough to the peak of the curve. The last data points of treatment were replotted.

To address the physiological relevance of the observations in immortalized N44 cells, I further tested the clock-modulating effects of gAdn on primary hypothalamic neurons and organotypic MBH slices. I isolated primary embryonic hypothalamic neurons and lentivirally transduced them to express Bmal1-luc. Upon Dex synchronization, the primary hypothalamic neurons showed a robust circadian Bmal1-luc expression rhythm 
with a period length $24.45 \pm 0.15 \mathrm{hr}$ (means $\pm \mathrm{SEM}, \mathrm{n}=2$; Fig. 6 A). Treating the neurons at $\mathrm{hr} 68$ (around the nadir) or hr 83 (around the peak) after Dex synchronization with gAdn resulted in stable phase-advances or -delays, respectively, consistent with the directions of phase-shift in N44 cells, except that the responses were more pronounced in primary neurons (Fig. 6 B \& C). Multiple nuclei in the MBH show robust self-sustaining circadian rhythms (21). I therefore used the slices of the ARC/ME complex from Per2::LUCIFERASE (Per2-luc) reporter mice as a model to test for the phase-resetting effect of gAdn ex vivo (43). Preparation of these slices indeed showed robust circadian oscillations of Per2-luc rhythms over 5 days of measurement (Fig. 6 D). In line with the effects observed from N44 cells and isolated neurons, treatment with gAdn resulted in phase-shifts of the Per2-luc expression rhythm in a phase dependent manner relative to PBS treated slices (Fig $6 \mathrm{E}-\mathrm{I}$ ). Together, these data suggest that adiponectin is a bona fide MBH molecular clock modulator. 
A

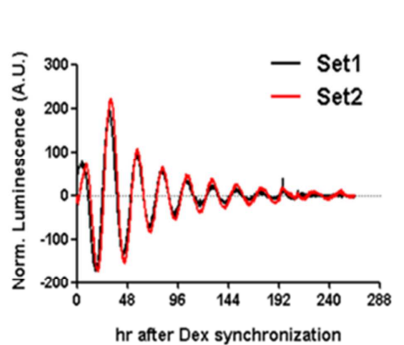

D

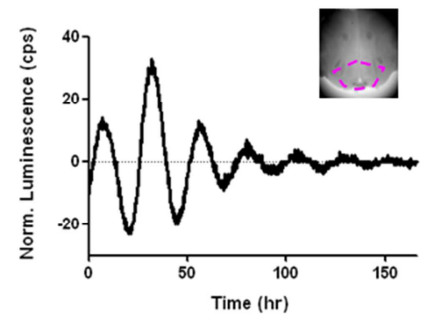

G

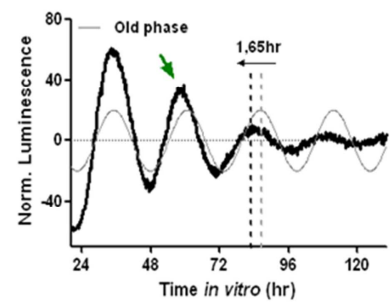

B

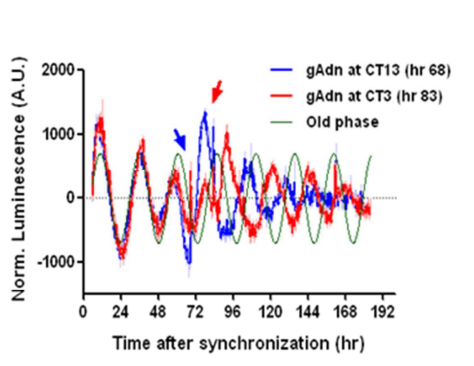

E

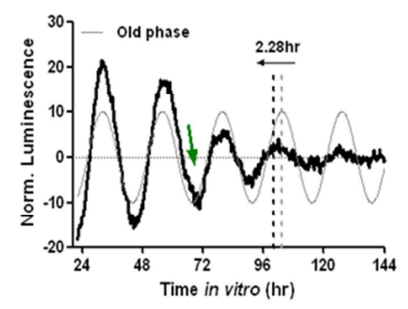

$\mathrm{H}$

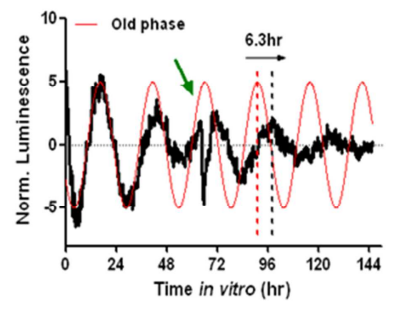

C

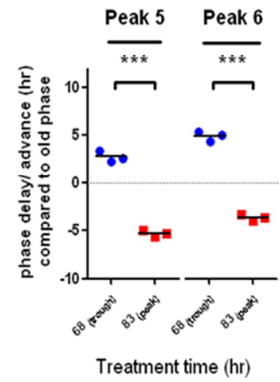

$\mathrm{F}$

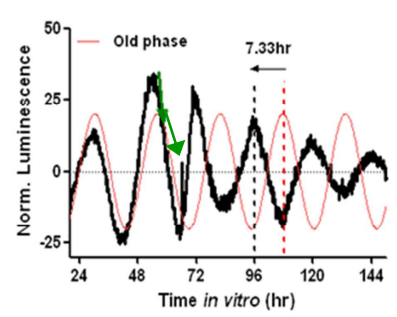

I

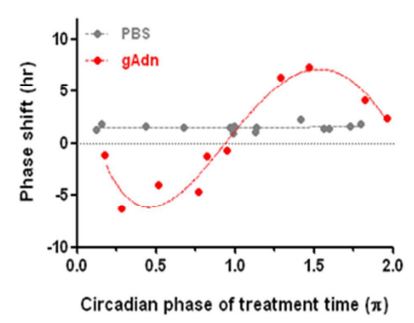

Figure 6. Adiponectin resets molecular clock in primary hypothalamic neurons and organotypic $\mathrm{MBH}$ slice cultures

(A) Representative normalized luminescence recordings of synchronized primary hypothalamic neurons expressing Bmal1-luc.

(B and C) gAdn resets cellular circadian rhythm in primary hypothalamic neurons expressing Bmal1-luc. (B) Normalized luminescence recordings of primary hypothalamic neurons treated with gAdn $(5 \mathrm{ug} / \mathrm{ml})$ at indicated time points after synchronization. (C) Quantification of gAdn-induced phase shifts of Bmal1-luc rhythms shown in $(H)(n=3)$.

(D) Representative normalized luminescence recording of arcuate nucleus/median eminence (ARC/ME) slices from Per2-luc mice. The highlighted region of the insert indicates the ARC/ME preparation brought for luminescence recording.

(E and F) Representative luminescence recordings of the ARC/ME slices of Per2-luc mice treated near the trough of the Per2-luc rhythm as indicated by the arrows with PBS (E) or $3 \mathrm{ug} / \mathrm{ml}$ gAdn (F).

(G and $\mathbf{H}$ ) Representative luminescence recordings of the ARC/ME slices of Per2-luc mice treated near the peak of the Per2-luc rhythm as indicated by the arrows with PBS (G) or $3 \mathrm{ug} / \mathrm{ml}$ gAdn $(\mathrm{H})$.

(I) Phase response curve of gAdn-induced phase shift of Per2-luc rhythms of ARC/ME slices. The treatment time was adjusted into radian of the circadian Per2-luc rhythm of slices. Note that the PBS treated slices regardless of the treatment time showed about 1-2.5 hr phase-advancement compared to the old phases which is due to the natural transition to free-running period when kept ex vivo. Each dot represents individual slice treated with either PBS or gAdn at indicated circadian phase.

Error bars indicate means \pm SEM. ${ }^{* *} p<0.001$, Student's t-test. 


\subsection{Characterization of the circadian phenotype of adiponectin deficient mice}

To investigate the physiological relevance of the $\mathrm{MBH}$ clock-modulating effects of adiponectin signaling in vivo, I used an established adiponectin deficient (Adipoq KO) mouse line $(44,45)$. To assess the circadian phenotype of Adipoq KO mice, a battery of circadian behavioral assays with running-wheels was performed. Adipoq KO mice have showed normal levels of locomotor activity compared to wild-type (WT) controls under both light-dark (LD) and constant darkness (DD) conditions (data not shown). Under LD, Adipoq KO mice displayed higher activities during the early dark phase followed by a compensatory decrease in the late dark phase (Fig. 7 A). However, Adipoq KO mice had no obvious defects on other circadian parameters of the activity rhythm that I have analyzed in LD, DD and LL (constant light ON) (Fig. 7 B - F). Interestingly, in a 6-hr phase advance experimental jet-lag paradigm, Adipoq KO mice readapted their activity rhythms faster to the new light/dark cycle than control animals (Fig. $7 \mathrm{G} \mathrm{\&} \mathrm{H).} \mathrm{These} \mathrm{data} \mathrm{suggest} \mathrm{that} \mathrm{adiponectin} \mathrm{is} \mathrm{not} \mathrm{a} \mathrm{major}$ regulator of the master circadian clock, but it may play a modulatory role in the entrainment of behavioral rhythms to shifts in the photic cycle. 
A

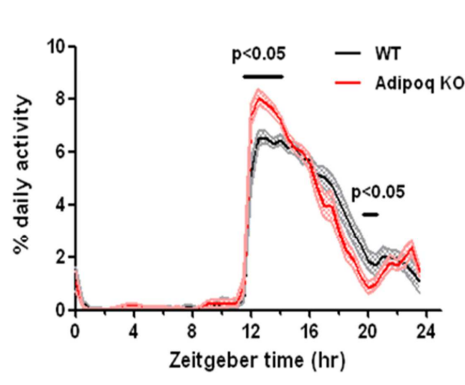

D

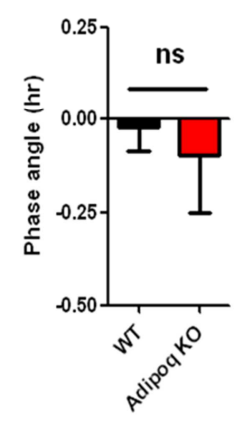

G

E
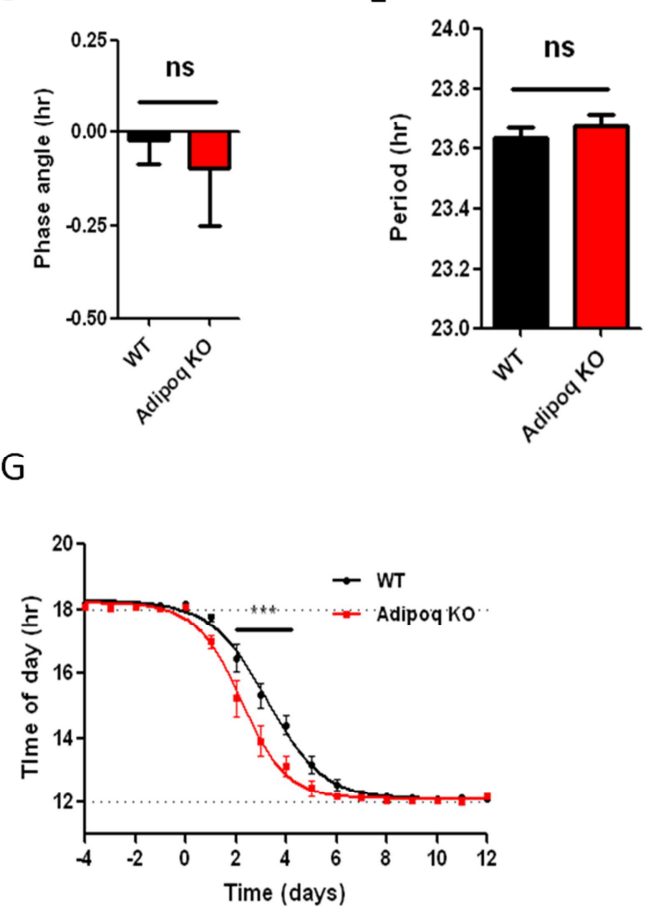

$\mathrm{H}$

C
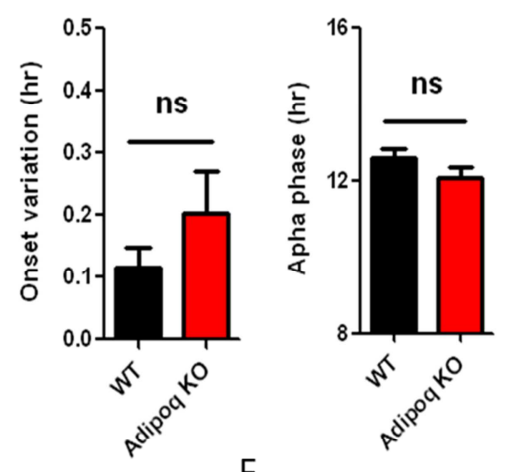

$\mathrm{F}$
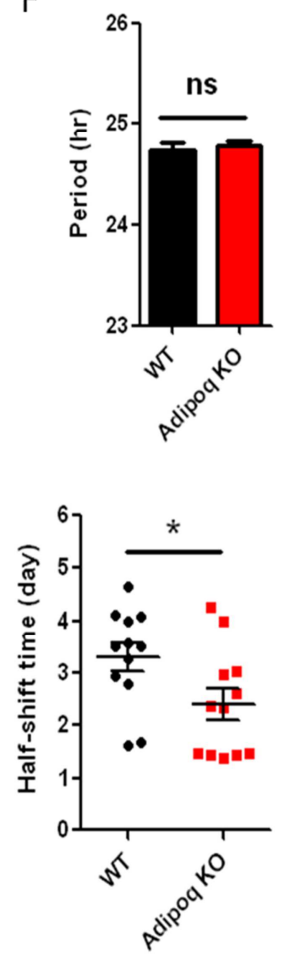

Figure 7. Characterization of the behavioral circadian phenotypes of Adipoq KO mice assessed by running wheels

(A) Daily activity profile integrated over 10 days in LD.

(B) Activity onset variation in LD.

(C) Alpha phase in LD.

(D) Phase angle with respect to light-OFF in LD.

(E) Average period over 10 days in DD.

(F) Average period over 10 days in LL.

(G) Activity onset under a $6 \mathrm{hr}$ phase-advance experimental jet-lag paradigm.

(H) Quantification of the half-shift time of the sigmoidal regressions in (G).

Error bars indicate means \pm SEM, $n=12$ per group, ${ }^{*} p<0.05,{ }^{* * *} p<0.001$, ns=not significant, Mann-Whitney test. 


\subsection{The role of the molecular clock in $\mathrm{MBH}$ functions}

As aforementioned, the molecular clocks residing in the $\mathrm{MBH}$ have been shown to regulate the local physiology in vitro, thus it appears that $\mathrm{MBH}$ clocks may contribute to the regulation of daily feeding patterns in vivo (46). Animals lacking components of the molecular clockwork show disrupted daily feeding behaviors (46; Table 1). To gain insights into how the circadian clock impinges on MBH functions, I analyzed the circadian regulation of mRNA levels of appetite-regulating neuropeptides (NP) in the MBH of WT and clock-deficient Bmal1 knockout (Bmal1 KO) mice. Loss of Bmal1 abolished the circadian expression rhythm of a well-established clock output gene, D site of albumin promoter (albumin D-box) binding protein (Dbp), along with drastic downregulation of its overall expression in the $\mathrm{MBH}$ (genotype effect, $\mathrm{F}=340.1, \mathrm{p}<0.0001$; Fig. $8 \mathrm{~A} \& \mathrm{~B}$ ). In the Bmal1 KO MBH, the 24-hr expression profiles of $\mathrm{Npy}(\mathrm{F}=11.96, \mathrm{p}=0.0032)$, Cart ( $\mathrm{F}=50.39$, $p<0.0001)$ and Pomc ( F=51.14, $p<0.0001)$ were significantly downregulated (Fig. $8 \mathrm{C}-\mathrm{F})$, suggesting that the circadian clock is an important regulator of appetite-regulating NP expression in vivo. Adiponectin is known to regulate a variety of physiological systems via its two cognate receptors, AdipoR1 and R2, which have been shown to have a broad range of tissue distribution in both the periphery and in the CNS $(39,40)$. To test the role of the circadian clock in the expression of AdipoRs in the MBH, I analyzed their mRNA levels in WT and Bmal1 KO mice. Consistent with the reported 24-hr expression profile of these two receptors in the periphery (47), I found that in WT mice Adipor2 expression showed a significant circadian rhythm $(\mathrm{P}<0.05$,

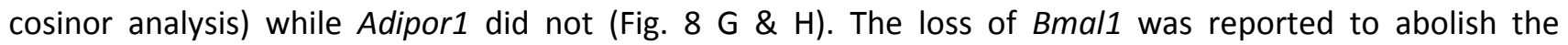
expression rhythms of Adipor2 and led to general downregulation of both Adipor1 and $-r 2$ in the periphery. In the $\mathrm{MBH}$, I observed that Bmal1 deficiency also abolished the circadian rhythm of Adipor2, but accentuated the overall expression of both Adipor1 $(F=22.09, p=0.0002)$ and $-r 2 \quad(F=18.29, p=0.0006)$, suggesting the existence of a tissue-specific regulatory mechanism for AdipoRs expression by the circadian clock. 

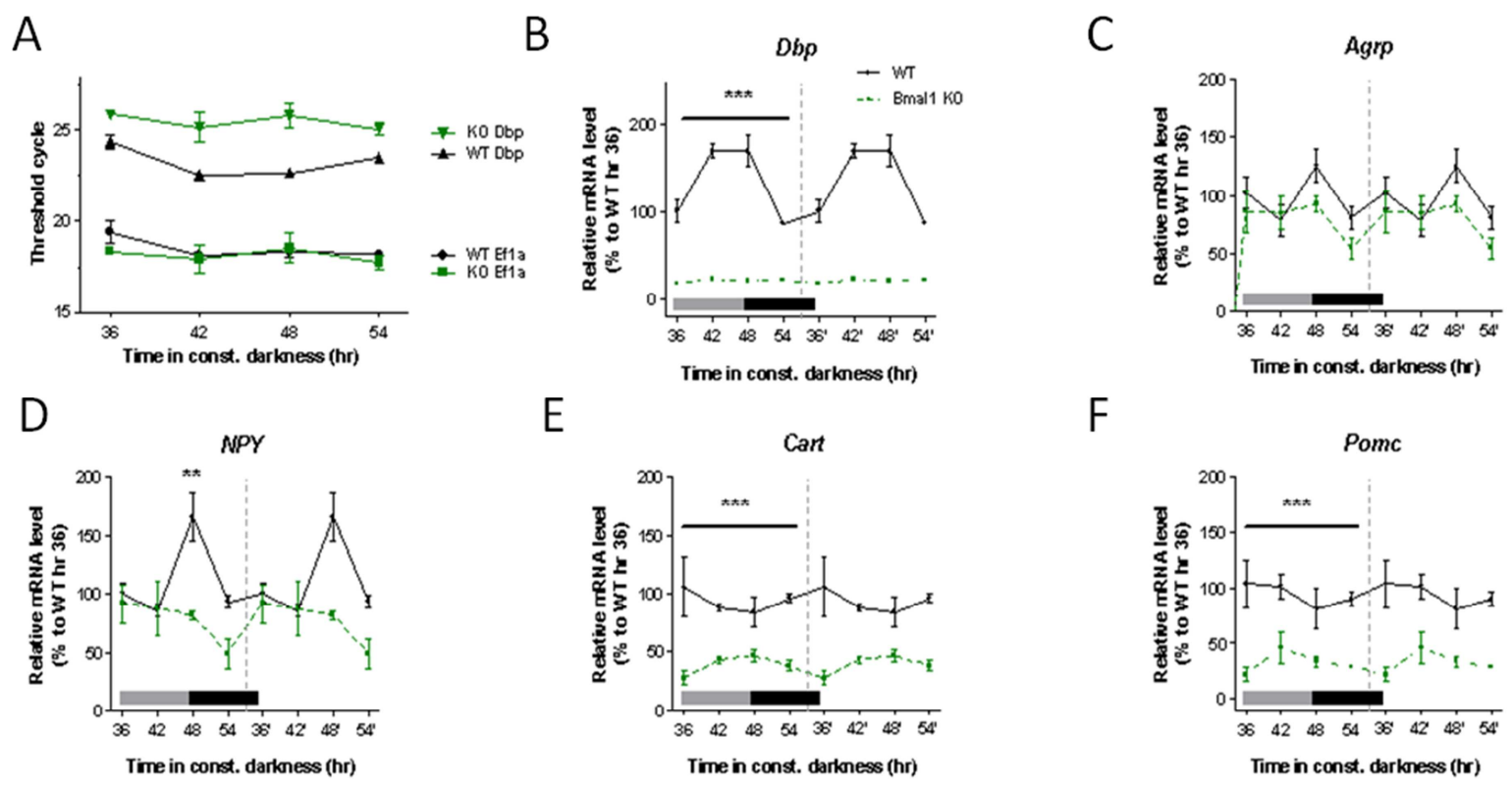

E

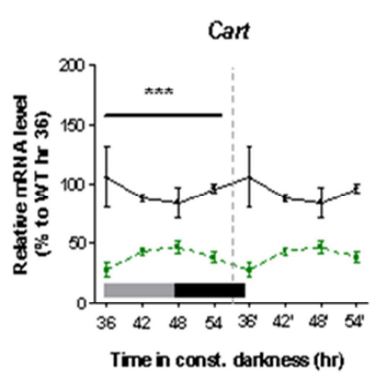

$\mathrm{F}$
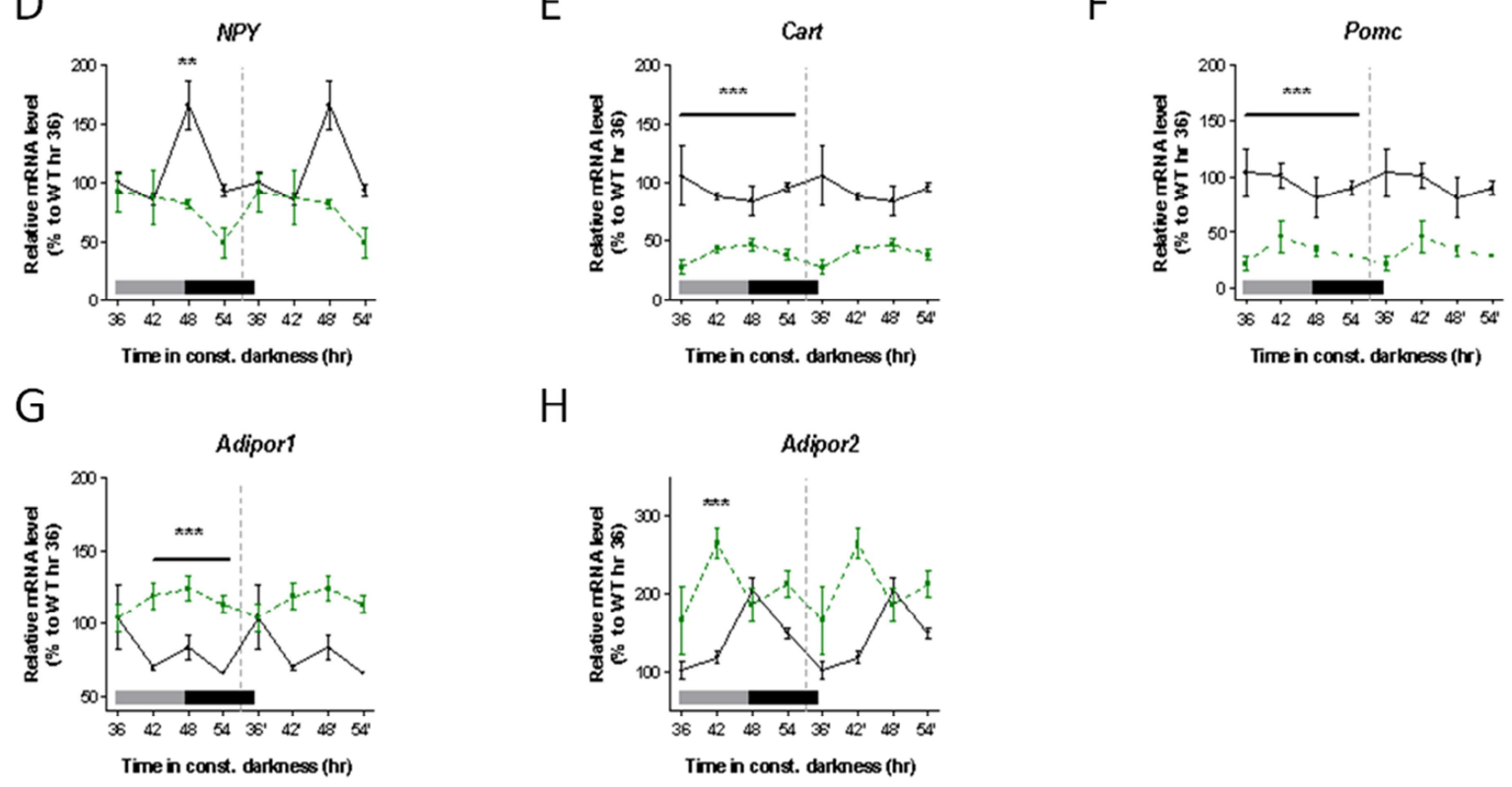

$\mathrm{H}$

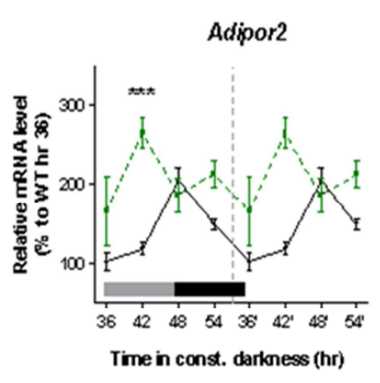

Figure 8. Characterization of appetite-regulating neuropeptides and adiponectin receptors $24 \mathrm{hr}$ expression profile in the MBH of WT and Bmal1 KO mice

qPCR analysis of the MBH of WT and Bmal1 KO mice released into constant darkness for indicated timespan.

(A) Threshold cycles of $D b p$ and housekeeping Ef1a genes in qPCR analysis.

(B - H) Double-plotted 24-hr mRNA expression profile of selected genes.

Error bars indicate means \pm SEM, $\mathrm{n}=3$ per time point, ${ }^{* *} \mathrm{p}<0.01,{ }^{* * *} \mathrm{p}<0.001$, Two-Way ANOVA with Bonferroni post-test.

\subsection{Adiponectin deficient mice have altered feeding rhythms}

Adipoq KO mice consumed comparable amounts of food per day as WT, however, they consumed significantly more food during the daytime (i.e. the light phase) in LD conditions and the subjective daytime (i.e. the normal rest phase) in DD. Further analysis revealed that Adipoq KO mice had a significantly 
dampened feeding rhythm in both LD (Fig. 9 A - C) and DD conditions (Fig. 9 D - F). To investigate if the loss of adiponectin could affect clock gene and appetite-regulating NP gene expression in the MBH, I analyzed the 24-hr expression profiles of corresponding transcripts in the MBH of Adipoq KO and WT mice. The circadian expression rhythms of Bmal1, Per2 and Dbp were significantly dampened in Adipoq KO mice (Fig. 9 G - I). Also, the diurnal expression oscillations of orexigenic NPs - AgRP, Npy and Hcrt - were markedly blunted, particularly during the subjective nighttime, in KO mice (Fig. $9 \mathrm{~J}-\mathrm{L}$ ). No significant effect was observed for anorexigenic NP transcripts - Cart and Pomc (Fig. 9 M \& N). 
A
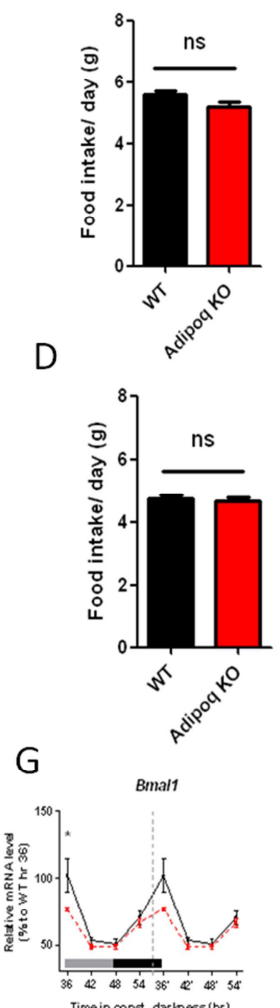

K

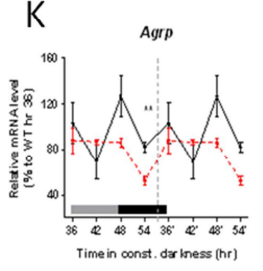

B
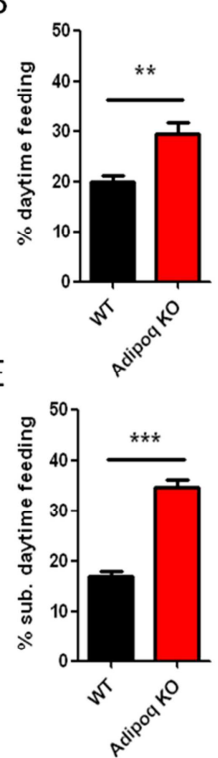

H

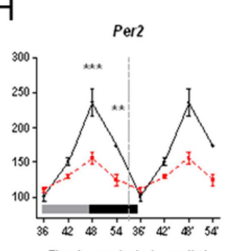

L

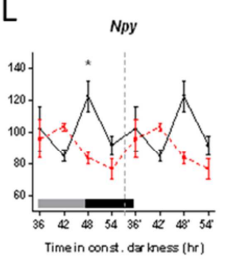

C

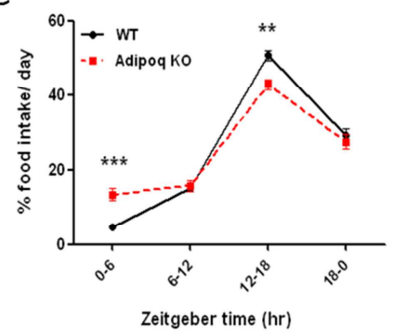

F

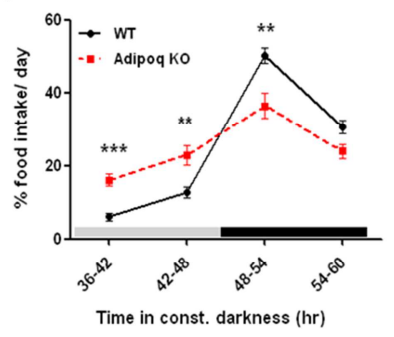

J Hert

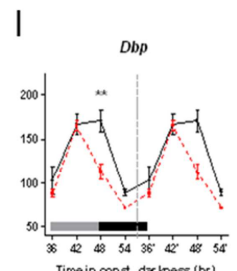

M

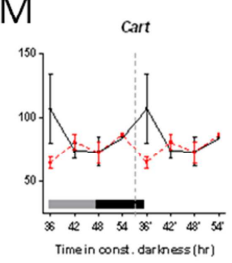

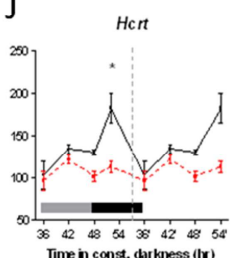

N

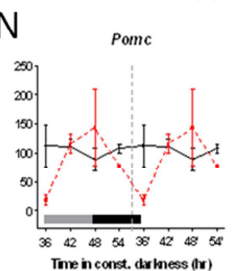

Figure 9. Adiponectin deficient mice exhibit dampened feeding rhythm and disrupted diurnal expression profile of clock genes and appetite-regulating neuropeptide genes in the $\mathrm{MBH}$

(A - F) Altered feeding rhythm in Adipoq KO mice under LD (A-C) and DD (D-F) conditions. (A and D) The daily food consumption under LD and DD conditions. ( $B$ and E) Percentage of daily food consumption during the light phase (LD) and subjective daytime (DD). (C and F) 24-hr food intake profile of WT and Adipoq KO mice under LD (C) and DD (F) conditions. Data shown are an average of 2 measurements of the same cohort of mice separated by about a week $(n=12)$.

(G - N) Double-plotted $24 \mathrm{hr}$ mRNA expression profiles of selected clock genes and appetite-regulating neuropeptide genes in the MBH of WT and Adipoq KO mice released into DD for indicated time span ( $n=3$ per time point).

Error bars indicate means \pm SEM. ${ }^{*} p<0.05,{ }^{*} p<0.01,{ }^{* * *} p<0.001$, Mann Whitney test for pairwise comparison and Two-Way ANOVA with Bonferroni post-test for profile data. 


\subsection{Tissue-specific effect of adiponectin on the circadian clocks in vivo}

To understand if loss of adiponectin also affects clock regulation in other tissues, I also analyzed the 24-hr expression profiles of clock genes in the prefrontal cortex (PFC), the liver and femur skeletal muscles in WT and Adipoq KO mice (Fig. 10). In the PFC, I observed a general dampening of the expression rhythms similar to those of the $\mathrm{MBH}$, albeit the timing of effects was different. Only minor changes in clock gene expression were observed in the liver and muscles, suggesting that the effect of adiponectin on the cellular clock may be tissue specific and apparently clocks residing in the CNS are more affected.
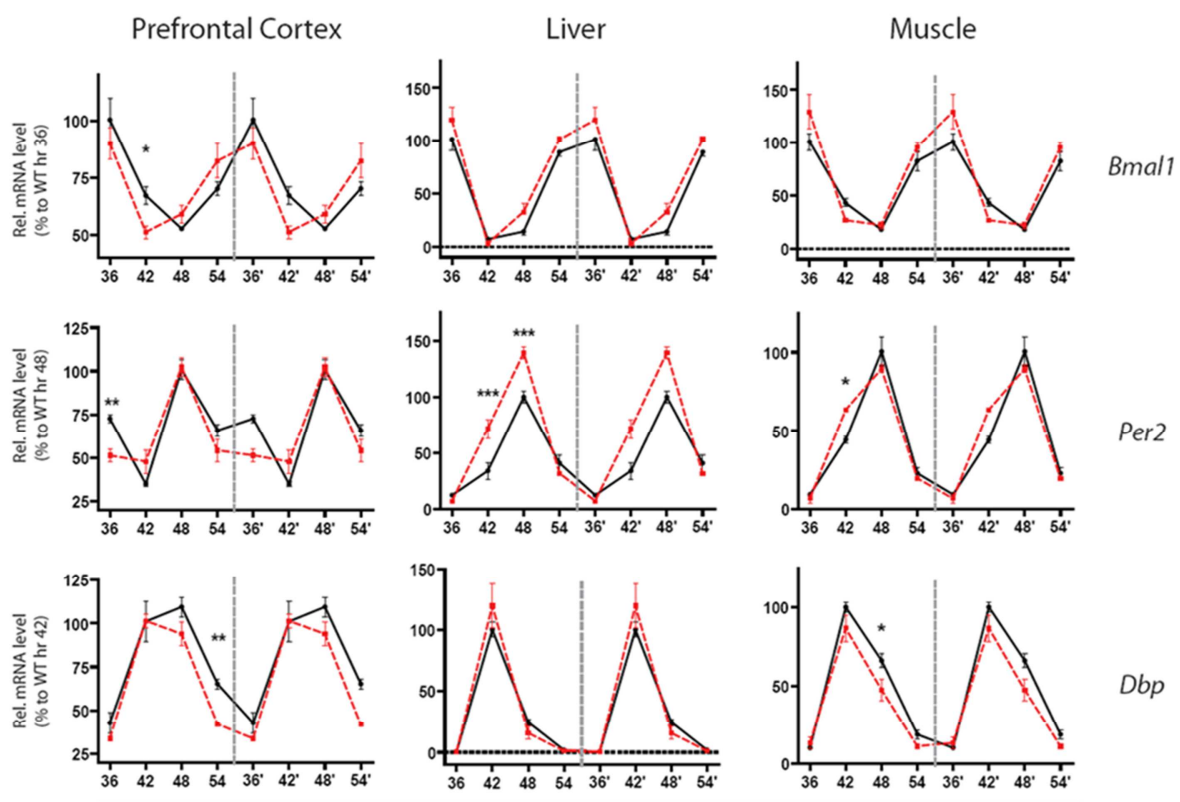

Time in constant darkness (hr)

\section{Figure 10. Tissue specific effects of adiponectin on Bmal1 expression in vivo}

Circadian expression profiles of selected clock genes in different tissues in WT and Adipoq KO mice released into DD for indicated time span ( $\mathrm{n}=3$ per time point). Error bars indicate means \pm SEM. ${ }^{*} \mathrm{p}<0.05,{ }^{*} \mathrm{p}<0.01$, Two-Way ANOVA with Bonferroni post-test

\subsection{Npy is a direct target of the molecular clock}

In both Bmal1 KO and Adipoq KO mice I observed a blunted diurnal expression profile of Npy, together with dampened MBH clock gene oscillations. Given the pivotal role of NPY neurons in appetite regulation, these data indicate that altered Npy expression may play a major role in the dampened feeding phenotype of 
Adipoq KO mice. In line with this claim, BMAL1 protein has been shown to rhythmically bind to the E-box elements of the Npy promoter in hypothalamic cells (22), suggesting that Npy is likely a direct target of BMAL1. To further interrogate this hypothesis, I used a short hairpin ribonucleic acid (shRNA) knock-down approach to demonstrate that reduction of Bmal1 expression in N44 cells resulted in Npy downregulation (Fig. 11 A \& B), indicating a positive relationship between Bmal1 and Npy expression. Moreover, using a Npy promoter end-point luciferase assay, I demonstrated that overexpressing BMAL1 and CLOCK activates the Npy promoter in a dose-dependent manner (Fig. 11 C). CLOCK/BMAL1's transactivating effect was abolished by co-expressing CRY1 (Fig. $11 \mathrm{D})$. Together, these data strongly indicate that NPY is a direct target of the molecular clockwork in the hypothalamus.
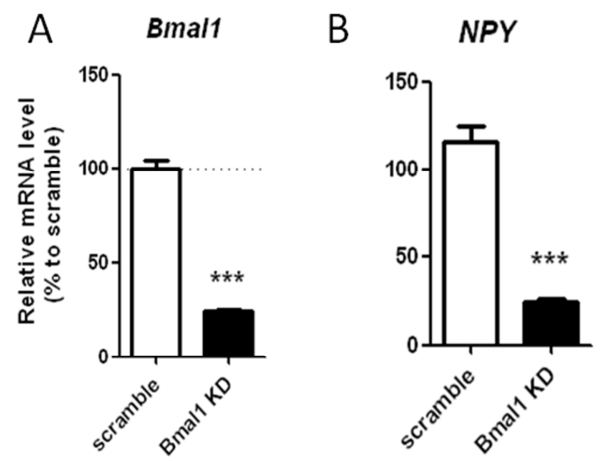

C

D
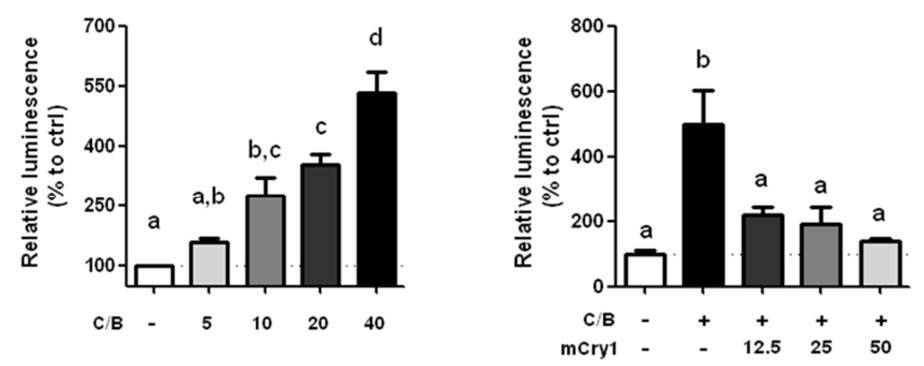

\section{Figure 11. Npy is a direct target of the molecular clock}

(A and B) qPCR analysis of Bmal1 (A) and NPY (B) expression N44 cells with shRNA knockdown of Bmal1 ( $\mathrm{n}=3)$.

(C and D) NPY promoter end-point luciferase reporter assay in HEK293T cells. (C) NPY promoter activity was activated by overexpressing CLOCK and BMAL1 in a dose- dependent manner ( $n=6)$. (D) The BMAL1/CLOCK's activating effect on NPY promoter was abolished by CRY1 overexpression ( $n=6)$.

Error bars indicate means \pm SEM. ${ }^{* * *} \mathrm{p}<0.001$, Student's $\mathrm{t}$-test. Groups denoted with different letters indicates statistical significance $(p<0.05)$, One-Way ANOVA with Tukey post-test. 


\subsection{Adiponectin regulates food anticipatory circadian rhythms}

When food availability is restricted to a particular time window of the day, circadian rhythms of animals will be entrained by the feeding schedule. At the level of behavior this is characterized by the development of FAA. The molecular clockwork in the central nervous system has been shown to play a role in regulating the FAA rhythm (48). In addition, metabolic hormones such as ghrelin and leptin can modulate FAA (19). To test if adiponectin is also involved in this behavioral re-adaptation, I challenged WT and Adipoq KO mice with a time-restricted feeding (RF) regime in which food availability was gradually confined to a 4-hr time window during the late rest phase (ZT 7-11). Locomotor activity within the 3-hr time window (ZT 4-7) preceeding feeding time was defined as FAA (Fig. 12 A). Of note, under this paradigm, I did not observe overt differences in well-being between WT and Adipoq KO mice as also reflected in body weight regulation and total activity levels (Fig. 12 B \& C). However, Adipoq KO mice showed a significantly delayed development of FAA (Day 3-5) compared to WT, but caught up with regard to total FAA from Day 6 on (Fig 12 D - F \& J). To discern if the effects of adiponectin on food anticipatory behavioral rhythms affect the food entrainable oscillator (FEO) I resumed the ad libitum feeding on Day 10 until ZT11 on Day 11 followed by food deprivation until ZT11 on Day 12. The ad libitum feeding largely abolished the FAA of WT and Adipoq KO mice on Day 11 (Fig. 12G, H \& J). The subsequent removal of food resurrected the FAA in both WT and Adipoq KO mice on Day 12, but the FAA of Adipoq KO mice was significantly reduced compared to that of WT animals (Fig. 12 I \& J). This data indicate that adiponectin directly impinges on the FEO system. In parallel to FAA I also measured food intake during Day 0-10 of the RF paradigm. Adipoq KO ate less during the early phase of the RF (Day 2 5), but then caught up later, similar to the dynamics observed for FAA (Fig. 12 K). Notably, on Day 2 when the food access was limited to the daytime, Adipoq KO mice consumed more food than WT, which is in line with what I observed under unchallenged conditions (Fig. 9 B). Together, I demonstrated that adiponectin contributes to the robustness of the FEO and promotes behavioral re-adaptation under a timed RF regime. 
A

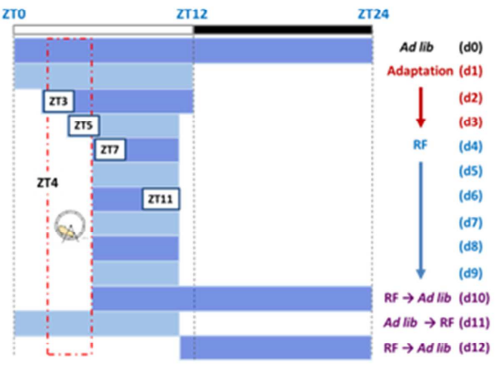

D

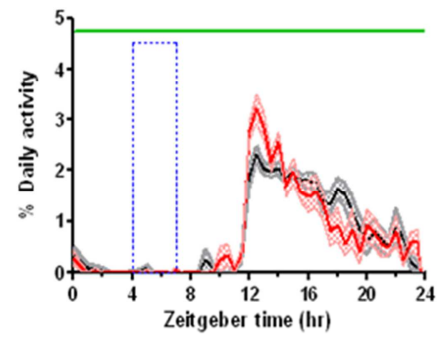

G

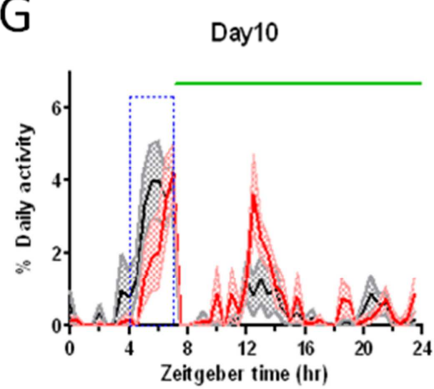

B

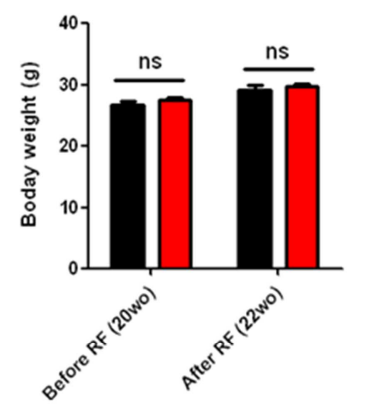

E

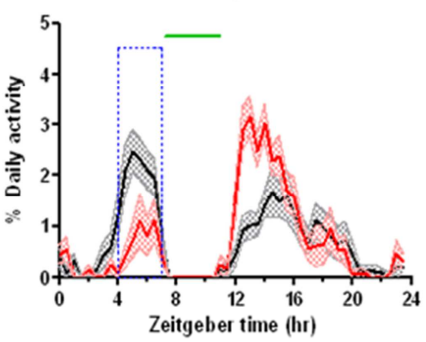

$\mathrm{H}$

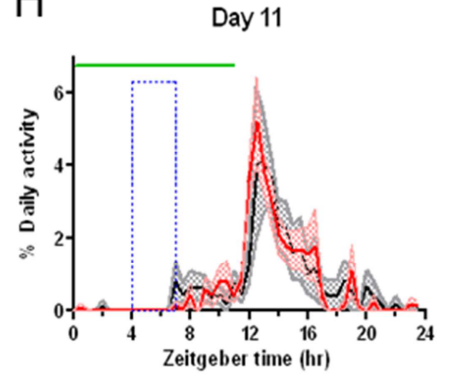

C

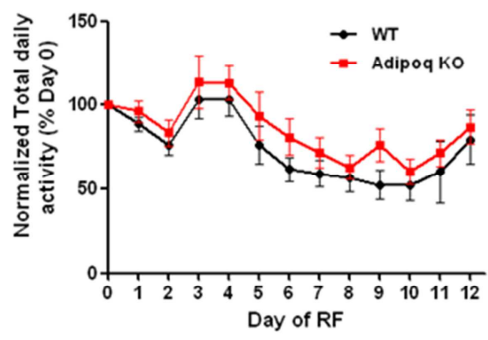

F Day 8

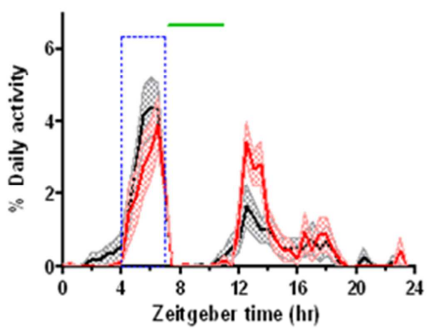

Day 12

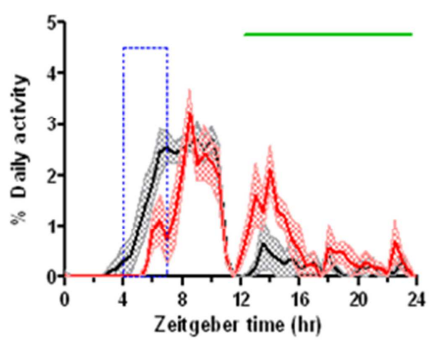

J

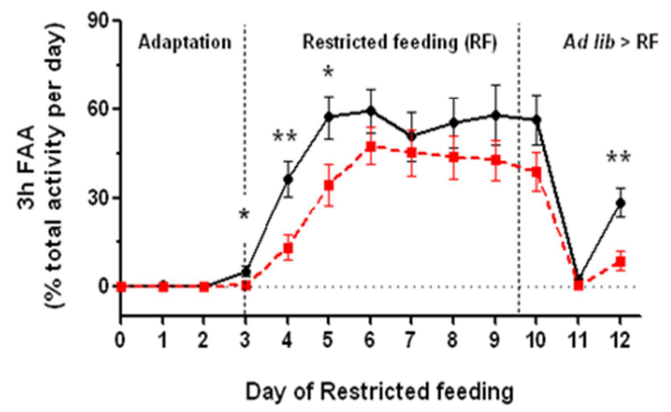

K

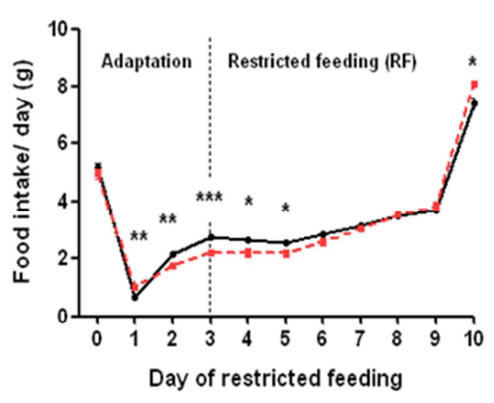

\section{Figure 12. Adiponectin regulates food anticipatory circadian activity rhythm}

(A) The time-restricted feeding (RF) regime used to induce FAA. The blue shade areas indicate the food-available time window. The rectangle with red broken border indicates the $3 \mathrm{hr}$ time window of the FAA.

(B) Body weight measured before and after the RF regime $(n=12)$.

(C) Normalized total daily activity over the course of RF regime ( $n=12$ on Day 1-10; $n=6$ on day 11-12, also for (D-K)).

(D - I) 24-hr activity profiles of individual day during the course of RF regime. The green bars overhead indicate feeding time; the blue rectangular windows indicate the $3 \mathrm{hr}$ FAA measured 
(J) Quantification of the 3hr FAA over the course of RF regime

(K) Total daily food consumption over the course of RF regime.

Error bars indicate means \pm SEM. ${ }^{*} p<0.05,{ }^{* *} p<0.01,{ }^{* * *} p<0.001$, Mann Whitney test.

\subsection{Adiponectin upregulates Bmal1 expression in the mediobasal basal hypothalamic neurons}

In the luminescence recordings of my phase-resetting experiments, I consistently observed that gAdn treatment resulted in an acute and long-lasting upregulation of the Bmal1-luc signal in the raw luminescence recordings independent of the phase of treatment (Fig. $4 \mathrm{H}$ ). In vivo, I observed that adiponectin deficiency led to a dampening of the molecular clock oscillation in the MBH (Fig. $9 \mathrm{G}-\mathrm{I}$ ). Together these findings suggested a positive effect of adiponectin on Bmal1 transcription. To confirm this, I compared gAdn treatment effects with that of other clock resetting agents on unsynchronized N44 Bmal1-luc cells. I found that gAdn treatment induced Bmal1-luc activity robustly over the course of $24 \mathrm{hr}$; in contrast, Fors and Dex did change the timing of luminescence peaks, but they rather led to a decrease of Bmal1-luc raw activity (Fig. $13 \mathrm{~A}-\mathrm{B})$. These data were consistent with the conclusion from the phase-resetting experiments that the mechanism of adiponectin clock resetting may differ from that of Fors and Dex, which both exert effects mainly via Per - but not Bmal1 - gene induction (5). gAdn treatment on N44 cells stably expressing luciferase under control of the constitutive mouse phosphoglycerate kinase 1 (Pgk) promoter (Pgk-luc) did not cause discernable changes in luciferase activity, supporting the specificity adiponectin's effects on Bmal1-luc expression (Fig. 13 C). Using qPCR I confirmed the upregulating effect of gAdn on endogenous Bmal1 expression at the mRNA level (Fig. 13 D) and by Western blot at the protein level (Fig. 13 F). Notably, Per2 was not acutely upregulated by gAdn (Fig. 13 E). Adiponectin is known to exist in different forms - in a globular form, as trimer, hexamer and high molecular weight (HMW) oligomers, all of which are formed via posttranslational processings of adiponectin peptides and display distinct affinities to AdipoR1 and R2 receptors $(49,50)$. Whilst the bacteria-expressed recombinant gAdn used so far represents a specific agonist of AdipoRs, treating unsynchronized N44 cells with full-length adiponectin (fAdn) expressed from mammalian cells (and comprising a mixture of different isoforms of adiponectin in a physiological ratio similarly resulted in a dose-dependent increase of Bmal1-luc activity, albeit with a lower efficacy compared 
to gAdn alone (Fig. $13 \mathrm{G} \mathrm{\&} \mathrm{H)}(38,50)$. To further confirm the physiological relevance of our observations, I performed gAdn treatment on synchronized Bmal1-luc expressing primary hypothalamic neurons around the peak time of the Bmal1-luc rhythm. Comparable to what I had observed in N44 cells, gAdn treatment of neurons acutely stimulated Bmal1-Luc activity (Fig $13 \mid \& J)$. These cell-based data are thus in line with the in vivo observations that adiponectin has an enhancing effect on Bmal1 expression. To investigate if the increase of circulating adiponectin could impinge on the MBH clock in vivo, with the help of my colleague Dr. Christiane Koch, we intravenously (i.v.) administered fAdn (1 $\mu \mathrm{g} / \mathrm{g}$ ) to Adipoq KO mice at ZT6 (in the middle of the descending phase of the MBH Bmal1 rhythm) via the tail vein. Bmal1 expression in the MBH of fAdn treated mice was significantly enhanced compared to PBS treated controls (Fig. 13K). To investigate if modulating endogenous central adiponectin bioavialability could impinge on $\mathrm{Bmal1}$ expression in the $\mathrm{MBH}, \mathrm{I}$ - again with the help of Dr. Koch - performed intracerebroventricular (i.c.v.) injections to centrally deliver anti-adiponectin antibodies ( $\alpha$-Adn) with the aim to antagonize central adiponectin signaling (39) on awake WT mice at ZT 21-22 (a few hours before the peak of MBH Bmal1 rhythms) under dim red illumination. a-Adn treatment significantly reduced Bmal1 expression in the $\mathrm{MBH}$ compared to unimmunized immunoglobulin G (IgG) controls (Fig. 13 L). In sum, in vitro and in vivo data provide compelling evidence that adiponectin signaling is a positive regulator of Bmal1 expression in the $\mathrm{MBH}$. 
A

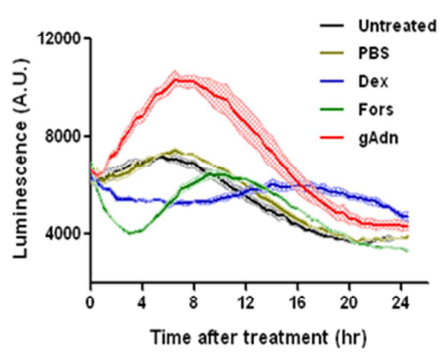

D

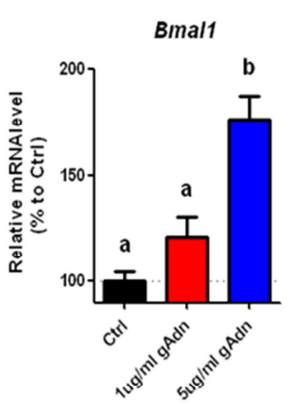

G

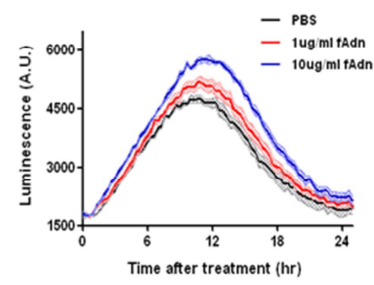

J

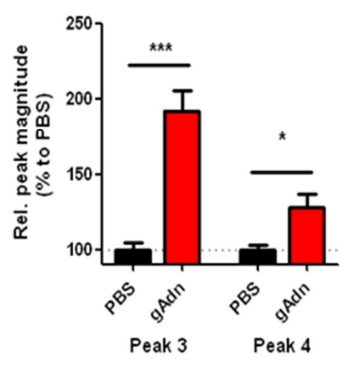

B

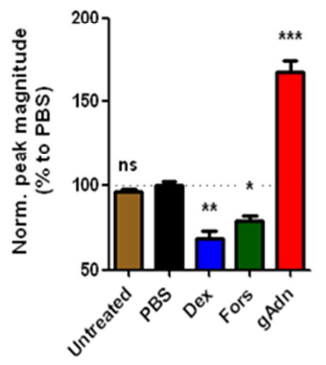

E

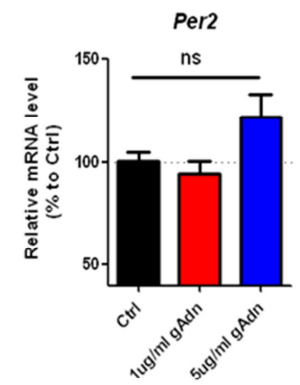

F

$\mathrm{H}$

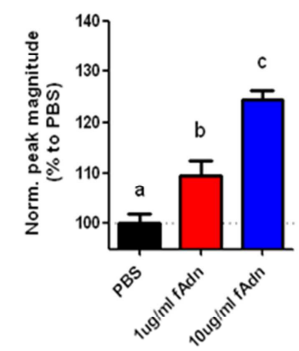

K

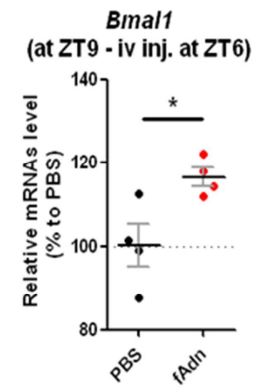

C
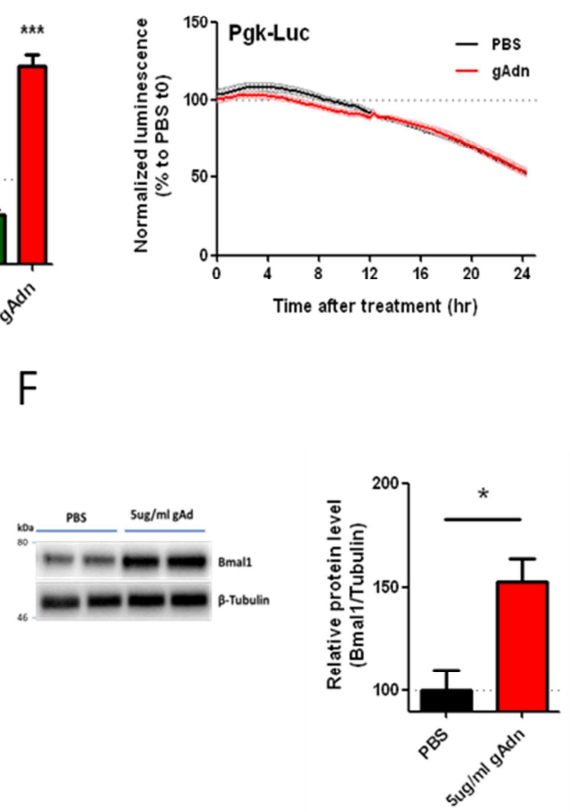

I

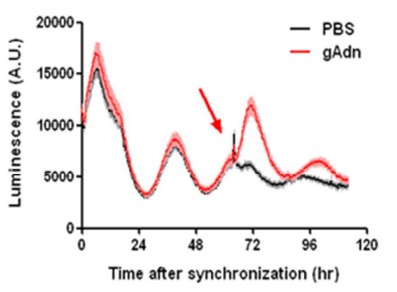

$\mathrm{L}$

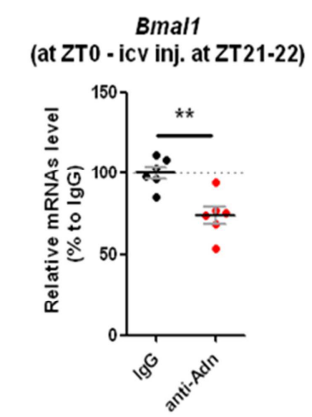

Figure 13. Adiponectin induces Bmal1 transcription

(A and B) gAdn (5ug/ml) and others clock-resetting agents treatment on unsynchronized N44 Bmal1-luc cells. (A) Raw bioluminescence recordings of the cells treated with drugs as indicated. (B) Quantification of the normalized peak luminescence magnitudes of recordings in $(A)(n=3)$.

(C) Normalized bioluminescence recordings of unsynchronized N44 cells stably expressing Pgk-luc treated with gAd (5ug/ml) (n=5).

(D and E) qPCR analysis of endogenous Bmal1 (F) and Per2 (G) expression in unsynchronized N44 cells treated with gAdn for $3 \mathrm{hr}$. 
(F) Immunoblot analysis of BMAL11 protein level in N44 cells $6 \mathrm{hr}$ after gAdn treatment (5ug/ml). $\beta$-tubulin was used as the loading control $(n=4)$.

(G and H) Full-length mammalian cells-expressed adiponectin (fAdn) treatment on unsynchronized N44 Bmal1-luc cells.

(G) Raw bioluminescence recordings of the cells treated with different doses of fAdn as indicated. (H) Quantification of the normalized peak luminescence magnitudes of recordings in $(G)(n=3)$.

(I and J) gAdn treatment on synchronized primary hypothalamic neurons expressing Bmal1-luc. (I) Raw luminescence recordings of Bmal1-luc expressing primary hypothalamic neurons treated with PBS or gAdn (5ug/ml) at the time as indicated by the arrow. (J) Quantification of the normalized peak magnitudes of the recordings shown in $(I)(n=3)$.

(K) qPCR analysis of Bmal1 expression in the MBH of Adipoq KO mice at ZT9 after i.v. injection with PBS or fAdn (1ug/g) at ZT6.

(L) qPCR analysis of Bmal1 expression in the MBH of WT mice at ZTO after i.c.v. administration with control IgG or anti-adiponectin antibody (0.6ug) at ZT21-22.

Error bars indicate means \pm SEM. ${ }^{*} \mathrm{p}<0.05,{ }^{* *} \mathrm{p}<0.01,{ }^{* * *} \mathrm{p}<0.001, \mathrm{~ns}=$ no significant difference. Groups denoted with different alphabets indicates statistical significance $(p<0.05)$. One-Way ANOVA with Bonferroni post-test for multi-groups analysis compared to the control group in (B). Student's t-test in (F) and (J). Mann Whitney test in (K) and (L). One-Way ANOVA with Tukey post-test in (D) and (E).

\subsection{Dose-dependency of adiponectin-induced phase resetting and Bmal1 induction}

The paradigm used for the peptide screen with synchronized N44 Bmal1-luc cells was proven to be a robust experimental setting which allows for simultaneously assessing the phase-resetting and Bmal1 induction effects of adiponectin (Fig. 4 A). To better determine phase- and dose-dependencies of adiponectin treatment, I treated synchronized N44 Bmal1-luc cells with gAdn at various doses and at roughly two opposite circadian phases - hr 13 (near the peak; Fig. 14 A) and hr 23 (near the trough; Fig. 14 B). Consistent to the data shown in Figure 4, the direction of the phase shift was opposite when the treatments were performed at opposite circadian phases. In contrast, the dose-dependency of the phase-resetting effect (i.e., the absolute phase shift) of gAdn did not depend on treatment phase (Fig 14 B \& E). Similarly, gAdn could also upregulate Bmal1-luc in a dose-dependent manner regardless of the circadian phase of the treatment (Fig. 14 C \& F). These data shed light on the mechanistic nature of the gAdn-induced clock-modulating effects. 
A

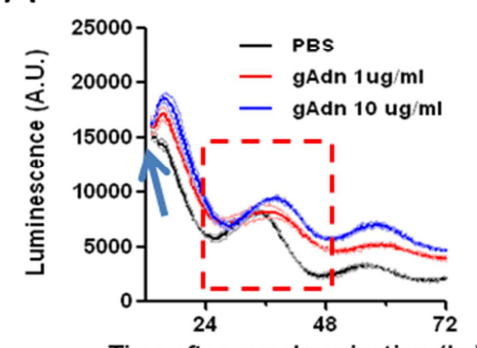

Time after synchronization (hr)

D

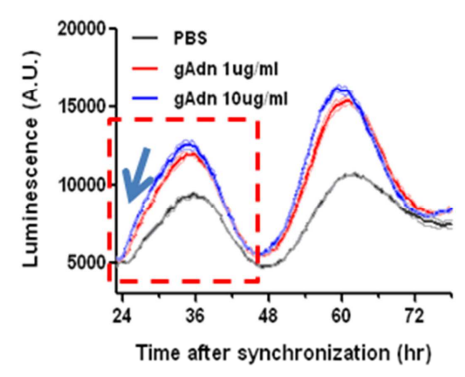

B

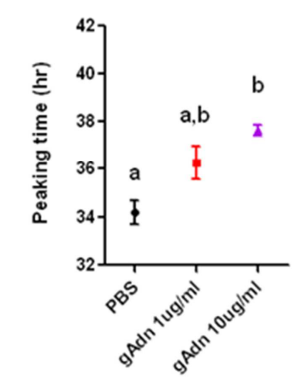

E

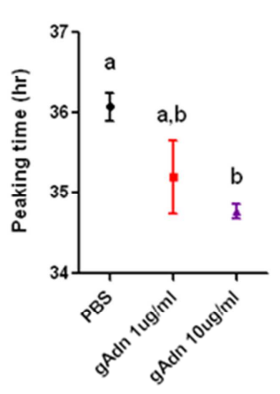

C

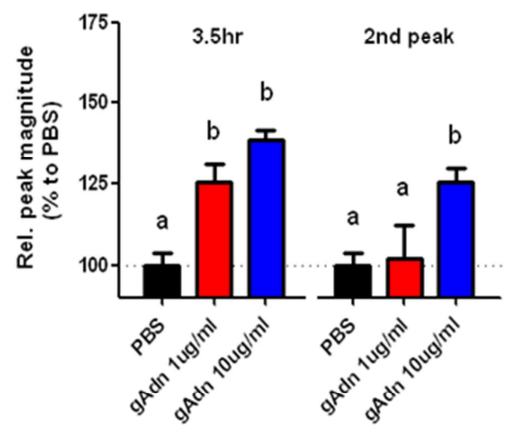

$\mathrm{F}$

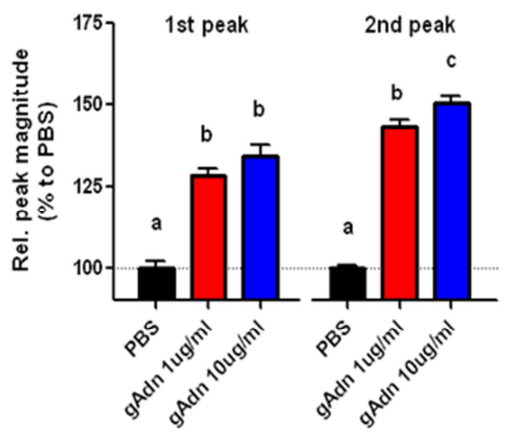

\section{Figure 14. Dose-dependency of adiponectin-induced phase resetting and Bmal1 induction}

(A - C) Dose-dependent effects of adiponectin on synchronized N44 Bmal1-luc cells treated near the peak (hr 13). (A) Raw luminescence recordings of synchronized N44 Bmal1-luc cells treated with various doses of gAdn at hr13. (B) Analysis of the peak time of the 2nd circadian cycle (rectangle with red broken-line) of recordings as shown in (A). (C) Analysis of the peak magnitude of the 2 nd and 3 rd circadian cycle of recordings as shown in (A).

(D - F) Dose-dependent effects of adiponectin on synchronized N44 Bmal1-luc cells treated near the trough (hr 23). (D) Raw luminescence recordings of synchronized N44 Bmal1-luc cells treated with various doses of gAdn at hr23. (E) Analysis of the peak time of the 2 nd circadian cycle (rectangle with red broken-line) of recordings as shown in (D). (F) Analysis of the peak magnitude of the 2 nd and 3rd circadian cycle of recordings as shown in (D).

Error bars indicate means \pm SEM. Groups denoted with different alphabets indicate statistical significance $(p<0.05)$, One-Way ANOVA with Tukey post-test, ns=not significant. 


\subsection{Tissue-specific effects of adiponectin on the circadian clocks in vitro}

In vivo, adiponectin's clock-modulating effects were strongly tissue-specific. To delineate if this specificity occurs at the cellular level (i.e. is cell-type specific) or at the systemic level (such as influenced by systemic signals), I tested the acute Bmal1 inducing effect of gAdn in different cell-lines engineered to stably express Bmal1-luc. Under unsynchronized conditions, gAdn treatment also acutely stimulated Bmal1-luc activity in another mediobasal hypothalamic cell line, mHypoE N41 (Fig 15 B). In contrast, gAdn treatment failed to elicit discernable changes in Bmal1-luc expression in cell lines of fibroblast origin (Fig 15 C \& D). Interestingly, this tissue specificity cannot be explained by the absence of particular adiponectin receptors including T-cadherin, which presumably is a decoy receptor of adiponectin signaling in cardiac tissues (Fig. 15 E) (40). Together, this experiment revealed that the acute Bmal1 inducing effect of adiponectin appears to be cell-type specific, probably due to the specific wiring of the downstream signaling cascades of adiponectin in different cell types $(37,40,49)$.

A

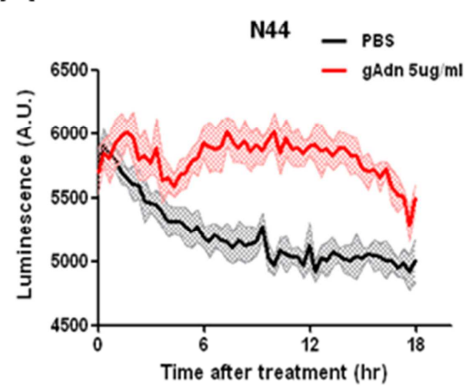

D

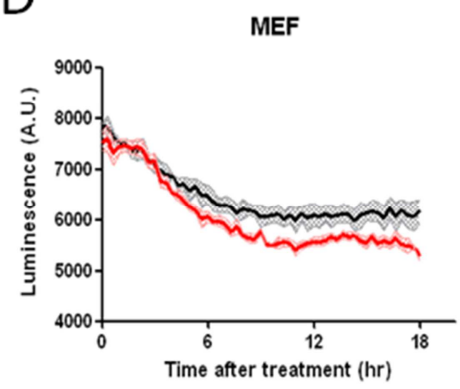

B

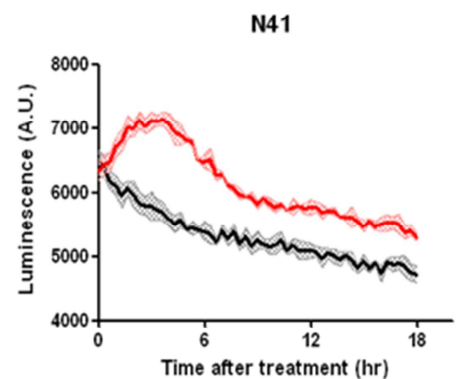

C

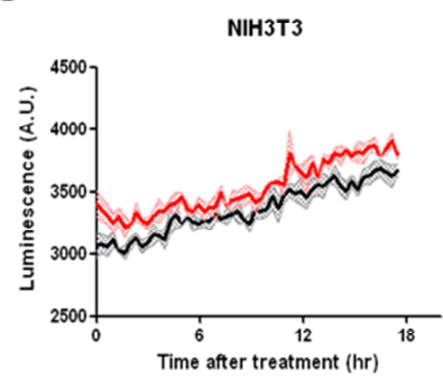

E

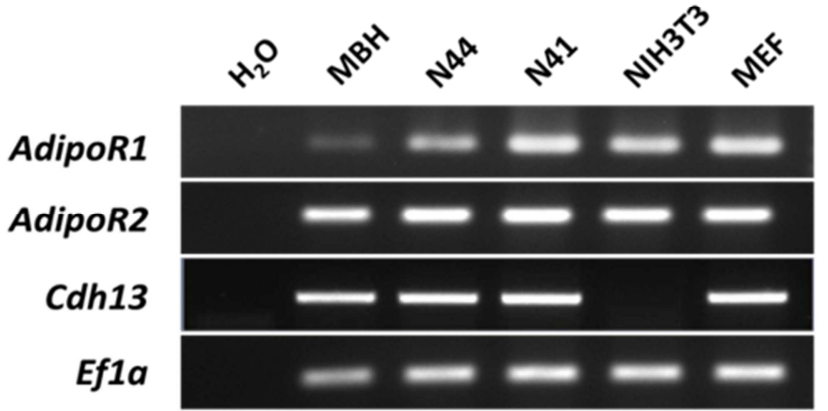

\section{Figure 15. Tissue specific effects of adiponectin on Bmal1 expression in vitro}

(A - D) Raw luminescence recordings of different unsynchronized cell lines stably expressing Bmal1-luc treated with gAdn ( $n=4)$.

(E) RT-PCR analysis of mRNA expression of adiponectin receptors in cell lines used in (B-E). 
Error bars indicate means \pm SEM.

\subsection{Differential roles of adiponectin receptors on in clock resetting}

In an early phase of this study, I noticed that the phase-resetting effect of gAdn treatment in N44 cells was observed only when cells were cultured in serum-free medium (Fig. 16 A - C). One potential explanation to this phenomenon is that adiponectin signaling is already saturated by the abundant presence of adiponectin molecules in serum-containing medium. If this would be the case, then a loss-of-function approach that knocks down individual components of the adiponectin signaling cascade in N44 cells maintained in serum-containing medium would be feasible to allow us to gain insight into the molecular mechanism of adiponectin clock resetting effects. Using a shRNA approach I knocked down (KD) Adipor1 and -r2 individually to less than one third of the original level in N44 cells. While knocking down Adipor2 had no effect on the mRNA level of Adipor1, knocking down Adipor1 led to a simultaneous downregulation of Adipor2 (Fig. $16 \mathrm{D} \& \mathrm{E}$ ). This effect did not depend on a specific shRNA, but may actually reflect an Adipor1-dependent regulation of Adipor2 expression, as transduction with a second AdipoR1-targeting shRNA gave a similar result (Fig. 17 C \& D). Knocking down individual adiponectin receptors intriguingly resulted in differential effects on Bmal1 expression: AdipoR1 KD led to downregulation of Bmal1 while AdipoR2 KD had the opposite effect (Fig. 16 F). These observations were confirmed with a second set of shRNAs targeting distinct sequences of both Adipor1 and $-r 2$ transcripts (Fig $17 \mathrm{C}-\mathrm{H}$ ). Interestingly, when both Adipor1 and -r2 were knocked down simultaneously (R1R2 dKD), it appeared that the Bmal1 downregulating effect of AdipoR1 KD was dominating over the upregulating effect of the AdipoR2 KD (Fig. 16 E and Fig. 17 A \& B). I speculated that REV-ERB $\alpha$, a transcriptional repressor of Bmal1, may be involved in the differential effect of the two adiponectin receptors on Bmal1 expression. Knocking down either Adipor1 or $-r 2$ individually reduced the expression of $N r 1 d 1$ (encoding gene of REV-ERB $\alpha$ ) (Fig. 17 B). While the downregulation of Nr1d1 in AdipoR1 KD cells is likely secondary to the downregulation of Bmal1, the reduction of Nr1d1 in AdipoR2 KD cells could explain the observed Bmal1 upregulation in these cells (Fig. 17

B). To understand the role of adiponectin receptors in cellular circadian rhythms, luciferase activity in 
synchronized N44 Bmal1-luc cells cultured in serum-containing medium with individual AdipoR knockdown was recorded. Consistent with previous data, AdipoR1 KD led to lower overall luminescence magnitudes while AdipoR2 KD had the opposite effect. I also analyzed the timing of the first peak which is related to the response of the cellular clock to the synchronizing signal and the average period over 4 circadian cycles. AdipoR1 KD resulted in a phase advance of the first peak together with period lengthening during subsequent cycles while AdipoR2 KD had no significant effects on these two parameters (Fig. $16 \mathrm{H}-\mathrm{J}$ ). Together, these data indicate that AdipoR1 may mediate the clock-modulating effects of adiponectin in hypothalamic neurons. To further test this, I treated AdipoR1 KD and scramble shRNA transduced N44 Bmal1-luc cells cultured in serum free medium at $\mathrm{hr} 23$ after synchronization with gAdn to analyze phase-resetting and Bmal1 induction. AdipoR1 knockdown significantly reduced gAdn induced Bmal1 upregulation and phase advances (Fig $16 \mathrm{~K}-\mathrm{N}$ ). Together, these data lead us to conclude that AdipoR1 is the major receptor that mediates the clock-modulating effects of adiponectin in $\mathrm{MBH}$ neurons. 
A

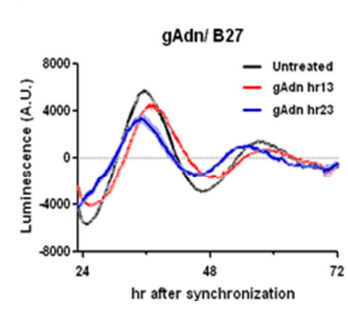

D

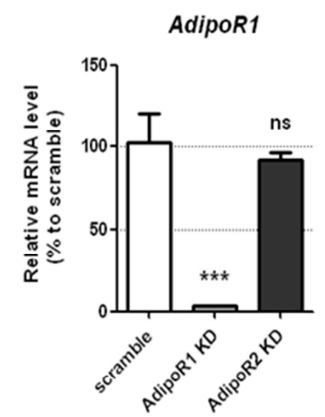

$\mathrm{H}$

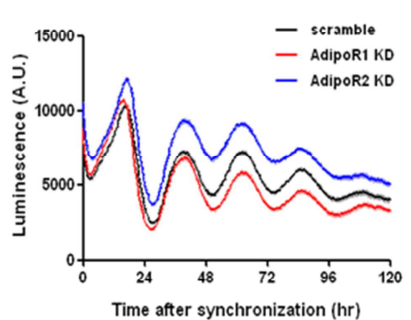

L

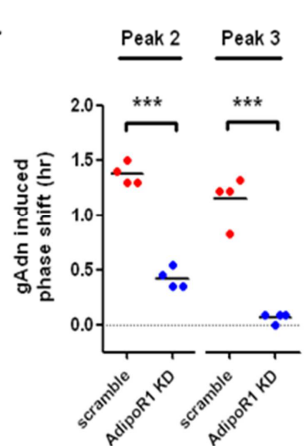

B

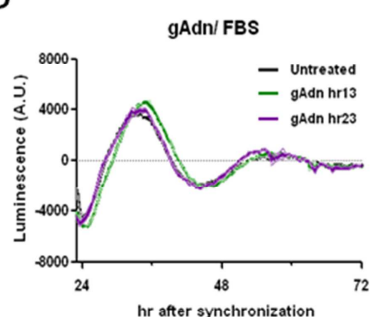

E

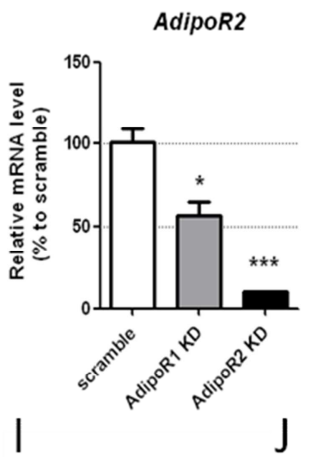

F

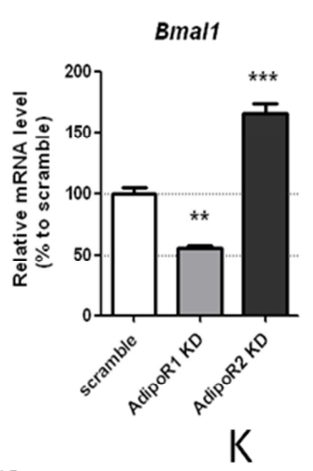

C

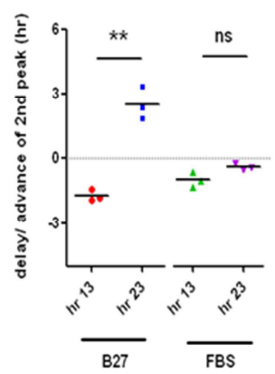

G

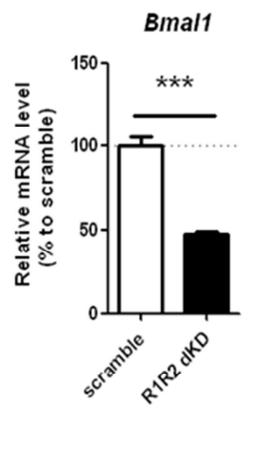

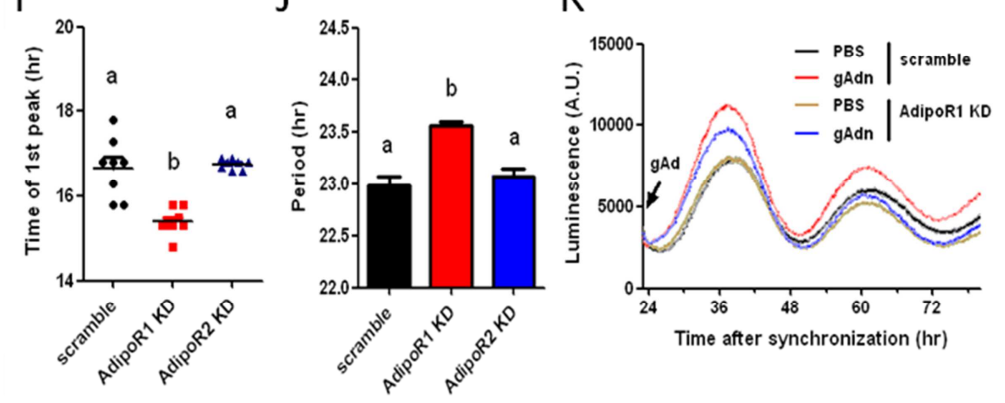
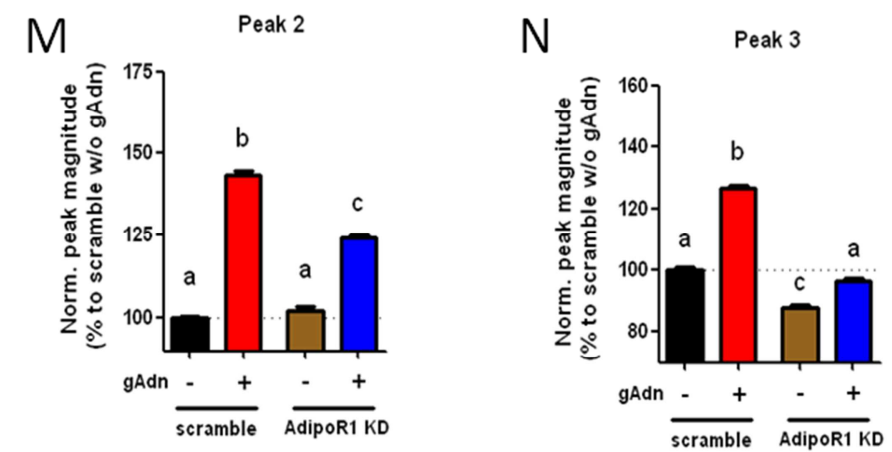

Figure 16. AdipoR1 mediates the clock modulating effects of adiponectin.

(A - C) Presence of serum abolishes the phase-shifting effect of gAdn in N44 cells. Normalized luminescence recordings of N44 cells cultured in B27 supplemented media (A) or in FBS containing media (B) treated with gAdn (3ug/ml) at indicated time points. (C) Quantification of the peak time of the 2 nd circadian cycle in (A) and (B) $(n=3)$.

(D - F) qPCR analysis of AdipoR1, AdipoR2 and Bmal1 expression in unsynchronized N44 cells with shRNA knockdown of AdipoR1 or AdipoR2 in serum-containing medium.

(G) qPCR analysis of Bmal1 expression in unsynchronized N44 cells with double knockdown of AdipoR1 and AdipoR2 in serum-containing medium. 
(H - J) Differential effects of shRNA knockdown of AdipoR1 and AdipoR2 on Bmal1-luc rhythm in synchronized N44 Bmal1-luc cells in serum-containing medium. (H) Raw luminescence recordings; (I) Peak time analysis of the first circadian cycle; (J) The average period length of the Bmal1-luc rhythms over 4 days of recording $(n=8)$.

(K - N) Effects of AdipoR1 knockdown on the phase-shifting and Bmal1 upregulating effect of gAdn treatment (5ug/ml, treated at near the trough ( $\mathrm{hr} 23$ ) of the 1st circadian cycle) in synchronized N44 Bmal1-luc cells $(n=4)$. (K) Raw luminescence recordings; Quantification of the gAdn induced phase-shift $(L)$ and the normalized peak magnitudes of the 2nd (M) and 3rd (N) circadian cycles in control and AdipoR1 KD cells.

Error bars indicate means \pm SEM. ${ }^{*} p<0.05,{ }^{* *} p<0.01,{ }^{* * *} p<0.001, \mathrm{~ns}=$ no significant difference. Groups denoted with different alphabets indicates statistical significance $(p<0.05)$. Student's t-test for pairwise comparison in $(C, G$ and $L)$. One-Way ANOVA with Bonferroni post-test for multi-groups analysis compared to the control group in (D-F). One-Way ANOVA with Tukey post-test in (I,J,M, \& N). 

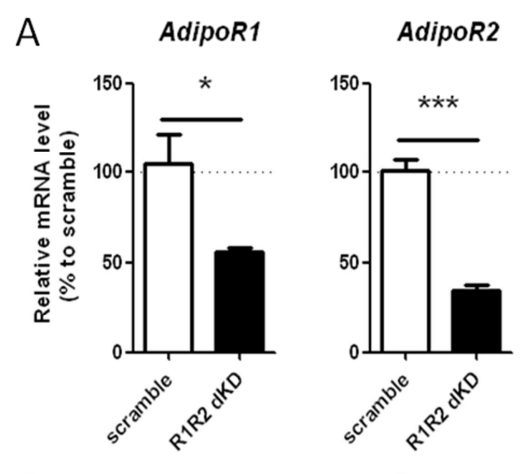

C

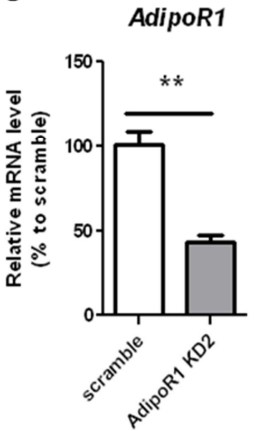

$\mathrm{F}$

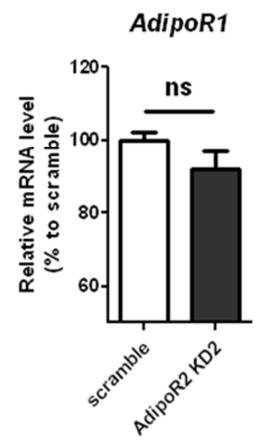

D

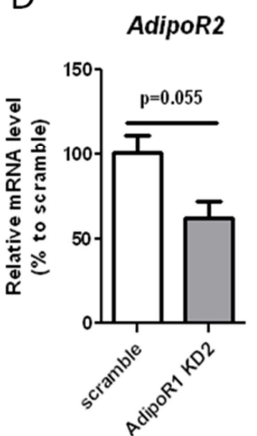

G

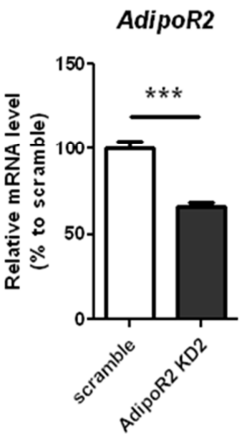

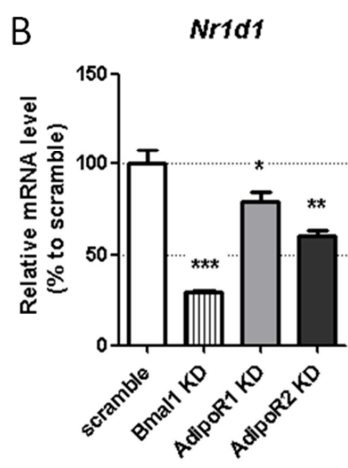

E

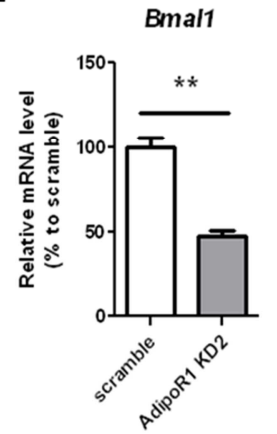

$\mathrm{H}$

Bmal1

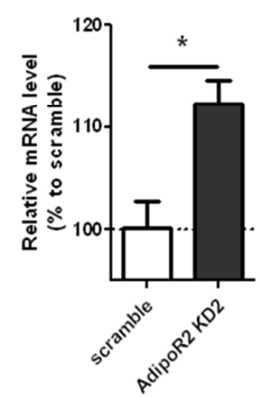

Figure 17. Supplementary data for the effects of shRNA knockdown of AdipoRs on the molecular clockwork in N44 cells.

(A) qPCR analysis of AdipoR1 and R2 expression in N44 cells with AdipoR1 and R2 double-knockdown ( $n=4)$.

(B) qPCR analysis of Nr1d1 expression in N44 cells with various shRNA knockdowns as indicated ( $n=3)$.

(C - E) qPCR analysis of selected genes in N44 cells with the 2nd AdipoR1 knockdown shRNA target sequence (AdipoR1 KD2) ( $n=4)$.

(F - H) qPCR analysis of selected genes in N44 cells with the 2nd AdipoR2 knockdown shRNA target sequence (AdipoR2 KD2) (n=4).

Error bars indicate means \pm SEM. ${ }^{*} \mathrm{p}<0.05,{ }^{*} \mathrm{p}<0.01, * * * \mathrm{p}<0.001$, Student's t-test for pairwise comparisons and One-Way ANOVA with Bonferroni post-test for multi-groups analysis compared to control in (B).

\subsection{PGC1 $\alpha$ mediates the clock-modulating effect of adiponectin in $\mathrm{MBH}$ neurons}

Recently, PGC1 $\alpha$ has been identified as a crucial mediator of AdipoR1 controlled metabolic effects in skeletal muscles (51). Interestingly, PGC1 $\alpha$ has also been identified as a modulator of the molecular clock acting via 
induction of Bmal1 expression (52). This mechanism highly resembles what I had observed for adiponectin. Therefore, I speculated that PGC1 $\alpha$ may play a crucial role in mediating adiponectin's clock-modulating effects. To test this, I investigated if alterations of adiponectin signaling could modify the expression of Pgc1 $\alpha$ in hypothalamic neurons. qPCR analysis of N44 cells cultured in serum-containing medium revealed that AdipoR1 KD resulted in dramatic reduction in Pgc1 $\alpha$ expression while AdipoR2 KD had no significant effect (Fig 18 A). These observations were consistent with previous reported findings on skeletal muscles (51). Conversely, gAdn treatment of N44 cells cultured in serum free medium resulted in an upregulation of Pgc1 $\alpha$ expression (Fig. 18 B) and enhanced the occupancy of an retinoic acid-related orphan receptor response element (RORE) in the Bmal1 promoter by PGC1 $\alpha$, but not in the $3^{\prime}$ untranslated region (3'-UTR) of the Bmal1 gene (Fig. $18 \mathrm{C}$ ). Thus, the adiponectin-AdipoR1-PGC1 $\alpha$ signaling cascade is conserved in $\mathrm{MBH}$ neurons similar to what has been shown in skeletal muscle (51). In vivo, I analyzed PGC1 $\alpha$ mRNA and protein levels in the MBH of WT and Adipoq KO mice. In WT mice, I did not observe significant circadian oscillations of Pgc1 $\alpha$ mRNA expression in the MBH (Fig. $18 \mathrm{D}$ ), but significant differences were observed in protein levels during the subjecting day and night (Fig. 18 E). Adiponectin deficiency resulted in significant downregulation of Pgc1 $\alpha$ mRNA at early subjective day and night (Fig. 18 D) and of PGC1 $\alpha$ protein during the subjective night time (Fig. $18 \mathrm{E}$ ). Thus, it can be concluded that adiponectin is a positive regulator of PGC1 $\alpha$ expression in the $\mathrm{MBH}$ in vitro and in vivo. To characterize the role of PGC1 $\alpha$ in regulating the molecular clockwork in $\mathrm{MBH}$ neurons, I investigated the cellular rhythms of synchronized N44 Bmal1-luc cells after Pgc1 $\alpha$ knockdown (PGC1 $\alpha$ KD) cultured in serum-containing medium. PGC1 $\alpha$ KD cells displayed cellular circadian phenotypes similar to those seen in AdipoR1 KD cells - dampened Bmal1-luc magnitudes, advanced phasing and lengthened period (Fig $18 \mathrm{~F}-\mathrm{H}$ ), indicating that the clock-modulating functions of PGC1 $\alpha$ and AdipoR1 use the same pathway. In synchronized N44 Bmal1-luc cells in serum-free medium, PGC1 $\alpha$ KD diminished the phase-resetting and Bmal1-inducing effects of gAdn treatment at hr 23 after synchronization, similar to what was observed in AdipoR1 KD cells (Fig. $18 \mathrm{I}$ - L). It has been shown that RAR-related orphan receptor alpha (ROR $\alpha$ ) activity is necessary for the circadian effects of PGC1 $\alpha$ (52). To test if ROR $\alpha$ is needed for adiponectin's circadian effects, N44 cells were pretreated with a recently established ROR $\alpha$ antagonist, 
VPR66 prior receiving gAdn treatment (53). I observed that VPR66 pretreatment abolished the Bmal1 inducing effect of gAdn (Fig. 19), further confirming the involvement of PGC1a-ROR $\alpha$ signaling in adiponectin mediated clock resetting in MBH neurons. 
A

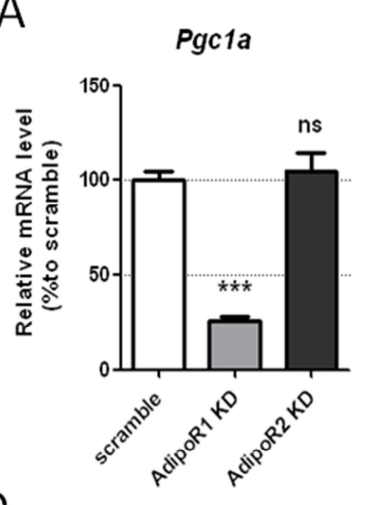

D

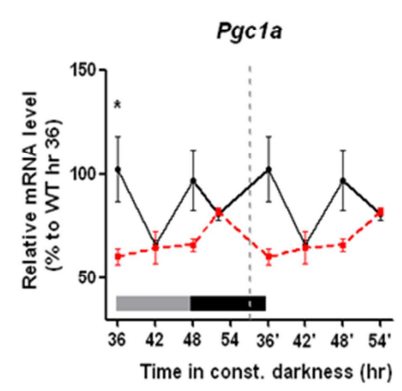

F

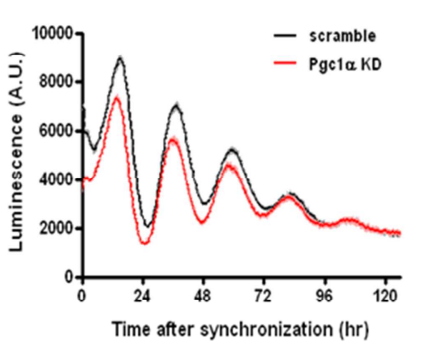

J

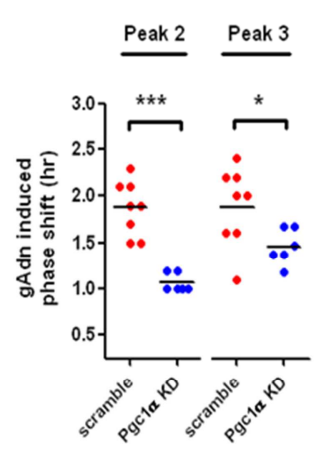

B

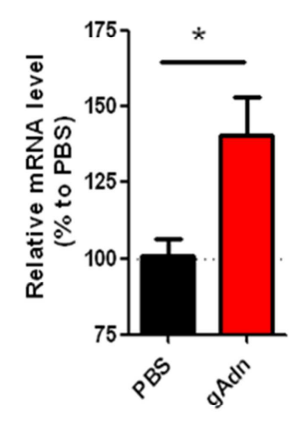

E

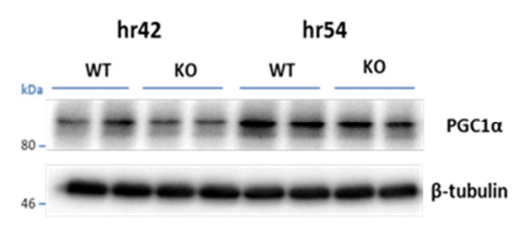

C BMal1 RORE

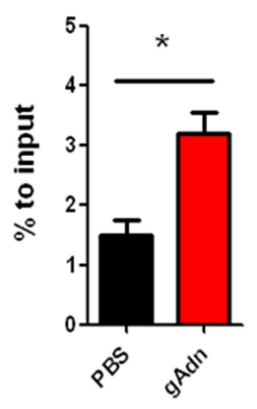

Bmal1 3'UTR
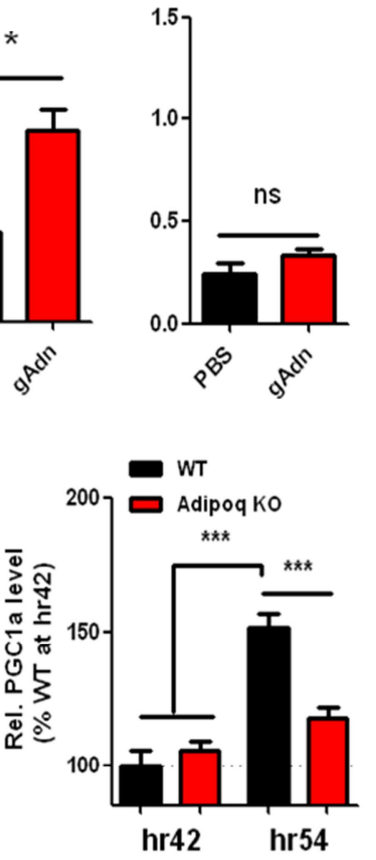

G

H I
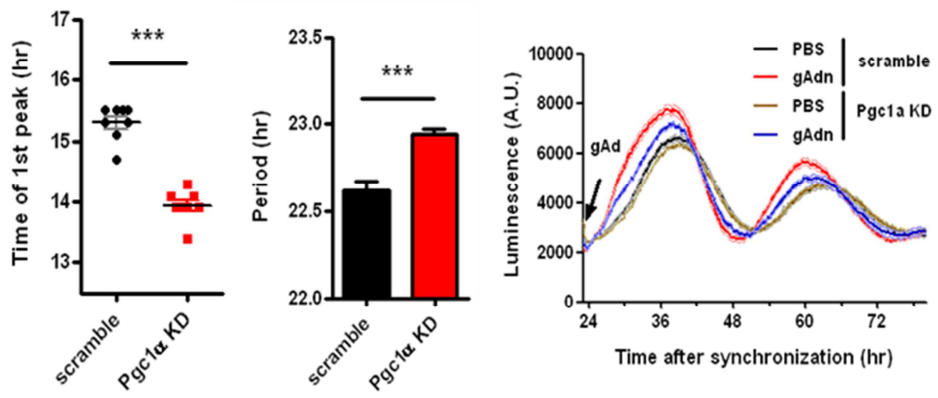

$\mathrm{K}$

Peak2

$\mathrm{L}$

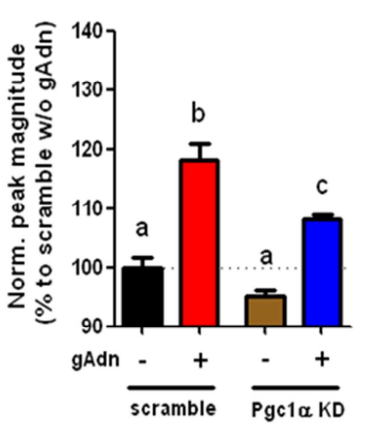

Peak3

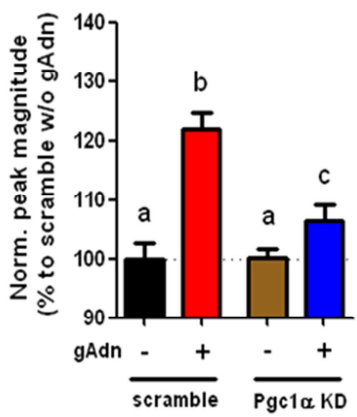

Figure 18. PGC1 $\alpha$ mediates clock modulating effect of Adiponectin via AdipoR1.

(A) qPCR analysis of Pgc1 $\alpha$ expression in unsynchronized N44 cells with shRNA knockdown of AdipoR1 or AdipoR2 in serum-containing medium $(n=3)$.

(B) qPCR analysis of Pgc1a expression in unsynchronized N44 cells after $2 \mathrm{hr}$ gAdn treatment (5ug/ml) ( $\mathrm{n}=4)$. 
(C) PGC1 $\alpha$ occupancy at the RORE sequence of the promoter and at the 3'UTR of the Bmal1 gene in N44 cells $2 \mathrm{hr}$ after gAdn treatment $(5 \mathrm{ug} / \mathrm{ml})(\mathrm{n}=3)$.

(D) Double-plotted 24-hr mRNA expression profile of Pgc1a in the MBH of mice released into DD for the indicated time span ( $\mathrm{n}=3$ per time point).

(E) Immunoblot analysis of PGC1 $\alpha$ protein level in the MBH from mice released into DD for the indicated time $s p a n(n=4)$.

(F - H) Effects of shRNA knockdown of Pgc1 $\alpha$ on the Bmal1-Luc rhythms in synchronized N44 cells in serum-containing medium. (F) Raw luminescence recordings; $(G)$ Quantification of the timing of the first peak; $(H)$ The average period of the cellular rhythm over 4 days of recording. $(n=8)$

(I - L) Effects of PGC1 $\alpha$ knockdown on the phase-shifting and Bmal1 upregulating effect of gAdn treatment $(5 \mathrm{ug} / \mathrm{ml}$, treated at near the trough ( $\mathrm{hr}$ 23) of the 1st circadian cycle) in synchronized N44 Bmal1-luc cells ( $\mathrm{n=4}$ ). (I) Raw luminescence recordings; Quantification of the gAdn induced phase-shift (J) and the normalized peak magnitudes of the 2nd (K) and 3rd (L) circadian cycles in control and PGC1 $\alpha$ knockdowned cells.

Error bars indicate means $\pm \mathrm{SEM} .{ }^{*} \mathrm{p}<0.05,{ }^{*} \mathrm{p}<0.01,{ }^{* * *} \mathrm{p}<0.001, \mathrm{~ns}=$ no significant difference. Groups denoted with different alphabets indicates statistical significance $(\mathrm{p}<0.05)$. Student's t-test for pairwise comparison. One-Way ANOVA with Bonferroni post-test for multi-groups analysis compared to the control group in (A). One-Way ANOVA with Tukey post-test in (E) and (J-L)..

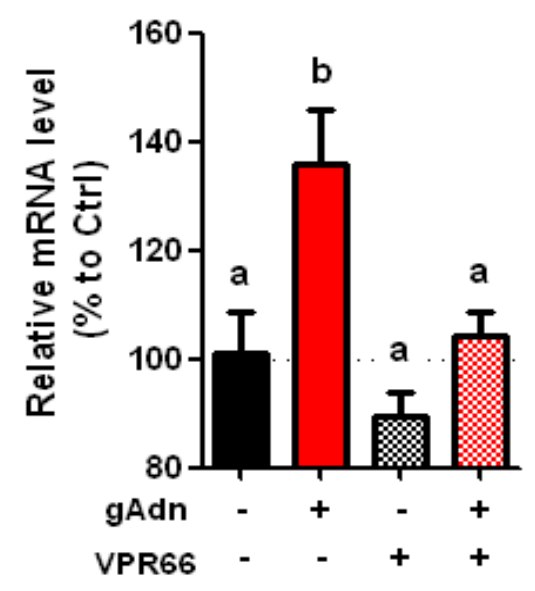

\section{Figure 19. Adiponectin's Bmal1 induction effect is dependent of ROR $\alpha$ activity}

qPCR analysis of Bmal1 expression in unsynchronized N44 cells pretreated with an antagonist of ROR $\alpha$ - VPR66 (5uM) $30 \mathrm{~min}$ before gAdn $(5 \mathrm{ug} / \mathrm{ml})$ treatment for $3 \mathrm{hrs}(\mathrm{n}=4)$. Groups denoted with different alphabets indicates statistical significance $(p<0.05)$. One-Way ANOVA with Tukey post-test

In the MBH, AMPK has been described as a mediator of central adiponectin signaling downstream of AdipoR1 (38). AMPK activates PGC1 $\alpha$ activity directly via phosphorylation and indirectly via a SIRT1-dependent pathway $(54,55)$. Interestingly, AMPK was also shown to directly modify the molecular 
clock by destabilizing CRY protein via phosphorylation (56). In N44 cells, gAdn treatment resulted in a transient phosphorylation of AMPK at Thr172 on the $\alpha$-subunit which is known to stimulate the kinase activity of AMPK (Fig. 20 A \& B) (57). Pre-treatment with an AMPK inhibitor, compound C, on the N44 Bmal1-luc cells significantly attenuated, but not fully abolished, gAdn induced Bmal1-luc activity (Fig. 20 C \& D), suggesting that AMPK also participates in adiponectin's clock modulating effect in MBH neurons. Together, conclude that the AdipoR1-AMPK-PGC1 $\alpha$-Bmal1 signaling cascade mediates adiponectin's circadian effects in mediobasal hypothalamic neurons.
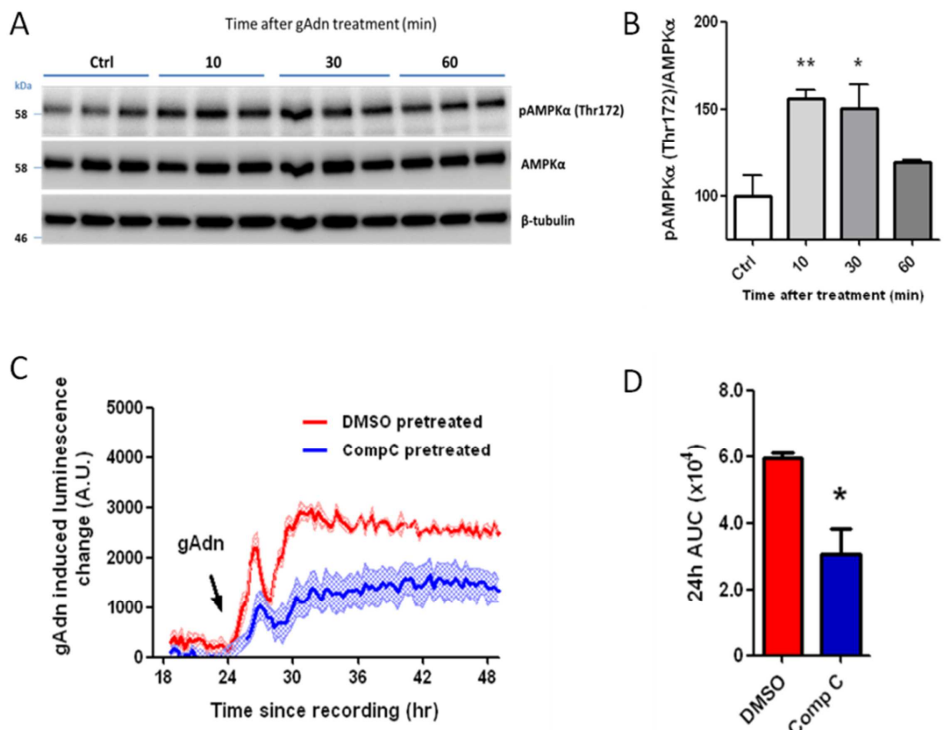

D

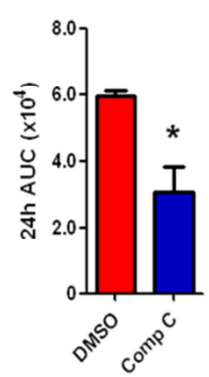

Figure 20. AMPK is involved in adiponectin induced Bmal1 upregulation.

(A and B) (A) Time-course immunoblot analysis of the phosphorylation of AMPK at Thr172 in N44 cells treated with gAdn (5ug/ml) (A). (B) Quantification of (A).

(C and D) (C) Normalized luminescence recordings of unsynchronized N44 Bmal1-Luc cells pretreated with an AMPK inhibitor compound C (5uM) 2 hrs before gAdn treatment (5ug/ml). (D) Quantification of the 24-hr area under the curve after gAdn treatment as shown in (C).

Error bars indicate means \pm SEM. ${ }^{*} p<0.05,{ }^{* *} p<0.01,{ }^{* * *} p<0.001$, One-Way ANOVA with Bonferroni post-test compared to control in (B) and Student's t-test in (D).

\subsection{The role of central adiponectin signaling in regulating food anticipatory circadian rhythms}

As mentioned, it has been shown that neuronal Bmal1 expression plays a role in regulating circadian FAA rhythms (48). Bmal1 expression in the DMH has been shown to contribute the FAA (58), but also (59). To 
characterize the mechanism of the FAA modulating effects of adiponectin, I analyzed the 24-hr profiles of plasma adiponectin and the mRNA levels of selected adiponectin signaling components, clock genes and appetite-regulating NP genes in the MBH of WT mice that were either fed ad libitum or submitted to the RF regime for 10 days as depicted in Figure 12A, except that food was also removed after ZT11 on Day 10. I observed that there was a modest but significant diurnal rhythm in plasma adiponectin $(p<0.001$, cosinor analysis) under ad libitum feeding conditions with peak-to-trough change of $27.62 \pm 8.39 \%$. On the other hand, the RF regime significantly reinforced this diurnal rhythm with a peak-to-trough change of $56.82 \pm 6.31$ $\%$ (means $\pm \mathrm{SEM}, \mathrm{n}=3$; Fig. $21 \mathrm{~A}$ ). Interestingly, the diurnal mRNA expression of adiponectin in adipose tissues was not significantly modified by RF, suggesting the existence of post-transcriptional mechanism mediating the influence of RF on diurnal blood oscillation (Fig. $21 \mathrm{~B}$ ). In the $\mathrm{MBH}$, the RF regime significantly enhanced the amplitude in the diurnal variation of transcripts of adiponectin signaling - Adipor1, Adipor2 and Pgc1a (Fig. $21 \mathrm{C}-\mathrm{E}$ ) and of clock gene rhythms - Bmal1, Per2, Dbp (Fig. $21 \mathrm{~F}-\mathrm{H}$ ). Moreover, the RF regime dramatically reorganized the 24-hr expression profiles of appetite-regulating NP genes in the MBH (Fig. $21 \mathrm{I}$ - L). Expression of AgRP was upregulated throughout the day and expression of Npy was upregulated during daytime (Fig. 21 I \& J). On the other hand, RF differentially regulated the expression of anorexigenic NPs - with Cart expression being upregulated during the early morning (Fig. $21 \mathrm{~K}$ ) while there was a trend for downregulation in Pomc during the day ( $F=4.16, p=0.0582$ ) (Fig. $21 \mathrm{~L}$ ). These data together with the impaired food anticipation phenotype in Adipoq KO mice suggest that reinforced central adiponectin signaling in the $\mathrm{MBH}$ may play a role in promoting the behavioral re-adaptation of the circadian system to temporally restricted feeding schedules. 
A

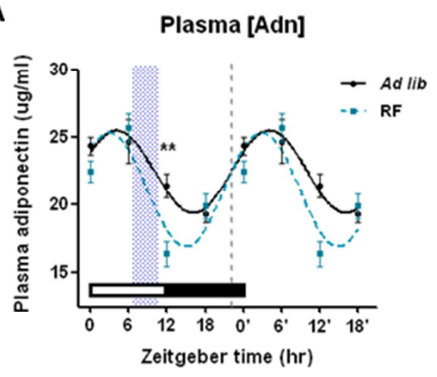

B

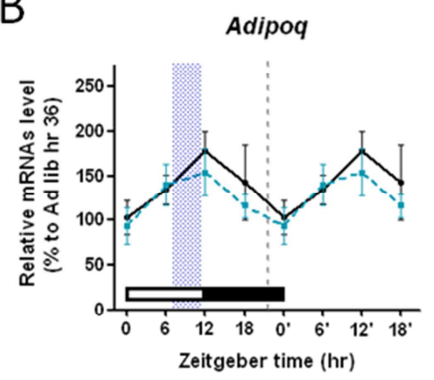

C

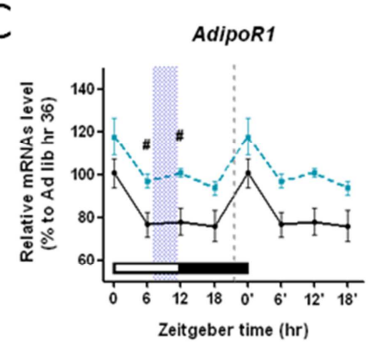

$\mathrm{F}$

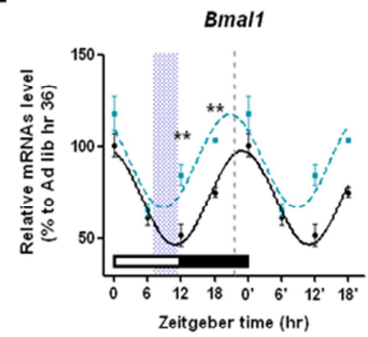

D

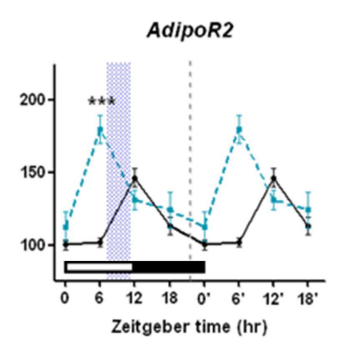

G

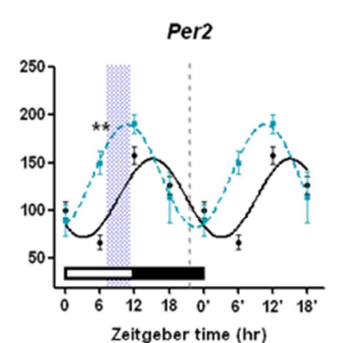

$\mathrm{E}$

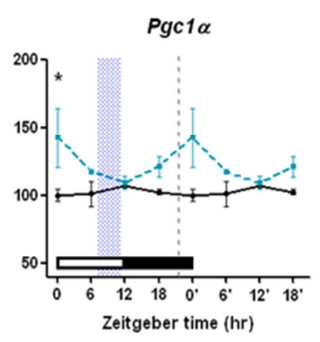

H

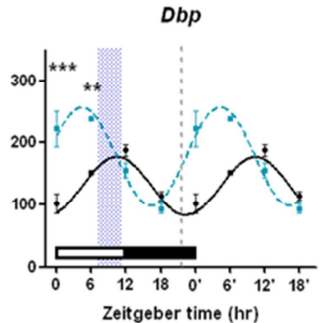

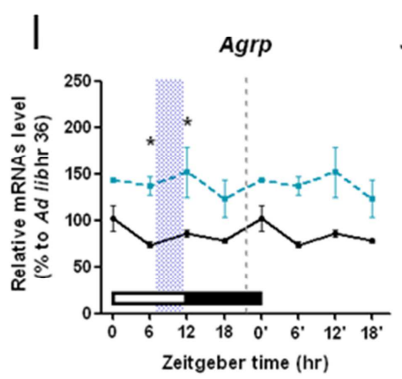
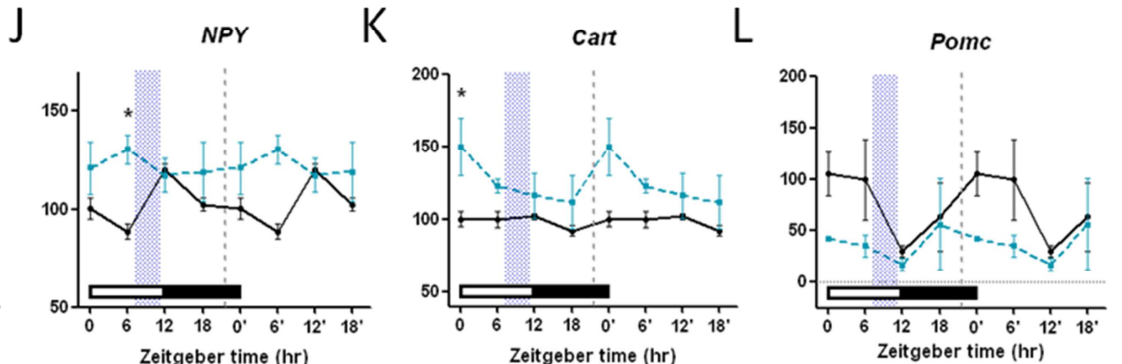

Figure 21. Regulation of the adiponectin signaling components in the $\mathrm{MBH}$ under time-restricted feeding regime

(A) Double-plotted 24-hr plasma adiponectin profile of WT mice under Ad libitum feeding and after 10 days in time-restricted feeding conditions as described in Fig. 3A under LD condition ( $n=3$ per time point).

(B) Double-plotted 24-hr mRNA expression profile of Adipoq gene in adipose tissues ( $\mathrm{n}=3$ per time point).

(C - L) Double-plotted 24-hr mRNA expression level of selected adiponectin signaling components (C-E), clock genes (F-H) and appetite regulating NP genes (I-L) in the MBH of WT mice under Ad libitum feeding and after 10 days in RF ( $n=3$ per time point).

Error bars indicate means \pm SEM, ${ }^{*} p<0.05,{ }^{* *} p<0.01,{ }^{* * *} p<0.001$, Two-Way ANOVA with Bonferroni post-test for profile data. 
To further strengthen the proposed role of central adiponectin signaling in food anticipatory circadian rhythms, and exclude that the observed phenotypes of Adipoq KO mice may result from general metabolic alterations as a consequence of non-circadian effects of adiponectin deficiency, in a collaboration with $\mathrm{Dr}$ Koch, we tested if acute pharmacological blockade of central adiponectin signaling in WT would similarly affect FAA. I submitted 2 groups of WT mice received i.c.v. administration of either DMSO vehicle or the ROR $\alpha$ antagonist-VPR66 at ZT0 on Day 4 and 5 under the RF protocol. VPR66 treated animals showed a delay in the development of FAA mirrored by reduced food intake from Day 5 to 7 (Fig $22 \mathrm{~A}-\mathrm{F}, \mathrm{H}$ \& I), similar to what was observed in Adipoq KO mice. Notably, I did not observe any significant reduction of total daily activity in VPR66 treated mice, suggesting that the general well-being was not compromised by VPR66 treatment (Fig. $22 \mathrm{G}$ ). Thus, these data further support the role of central adiponectin signaling in the entrainment food anticipatory circadian rhythms. 

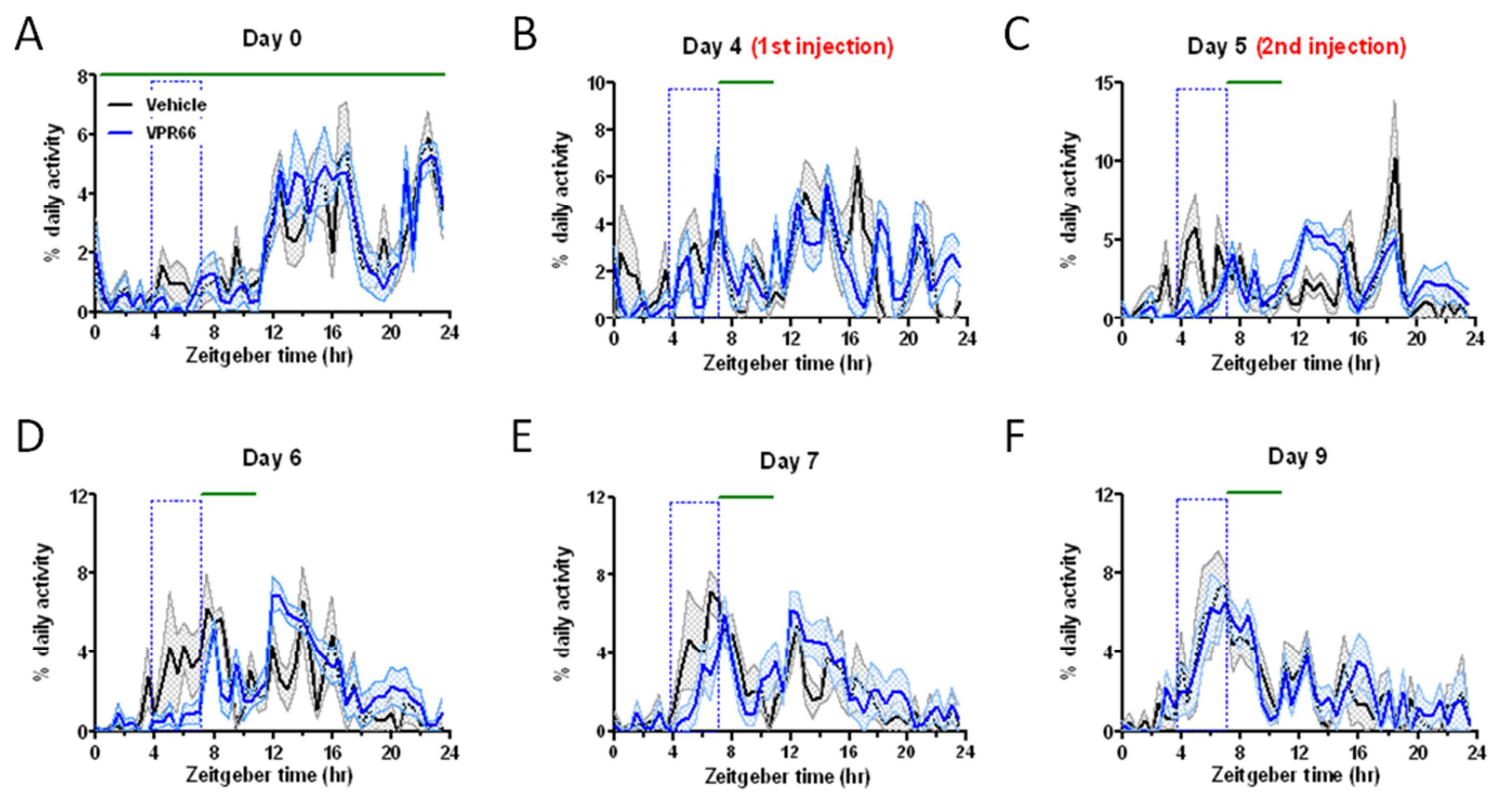

E

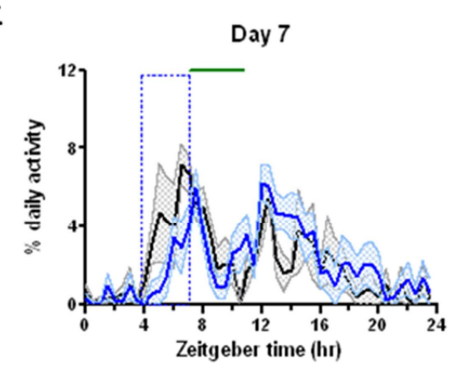

F

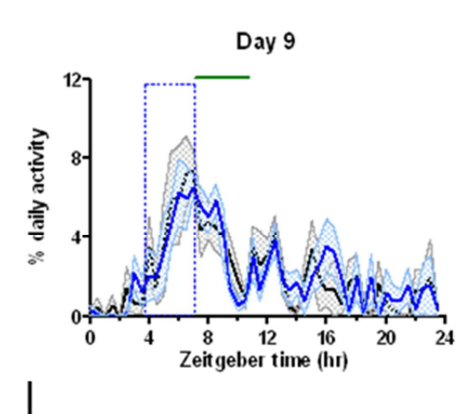

G

$\mathrm{H}$

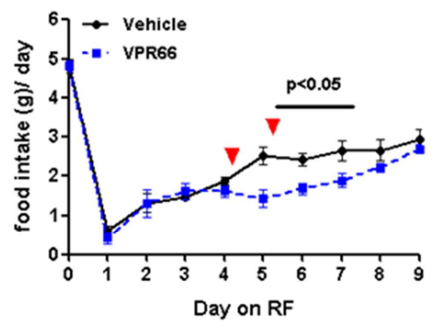

Figure 22. Central adiponectin signaling contributes to food anticipatory circadian activity rhythm.

i.c.v. administration of VPR66 (RORa inhibitor, $5 \mathrm{uM}$ ) at ZTO on day 4 and 5 (red arrows) under the RF regime impairs the development of FAA (assessed by infrared detectors).

(A-F) 24-hr activity profiles on individual days during the course of RF regime. The overhead green bars indicate feeding time; the blue rectangular windows indicate FAA measured. On day 4 (E) and 5 (F) mice were i.c.v. administered with 2 ul VPR66 or DMSO vehicle control at ZTO.

(G) Normalized total daily activity over the course of RF regime.

(H) Quantification of the 3hr FAA over the course of RF regime.

(I) Daily food consumption over the course of RF regime.

Error bars indicate means \pm SEM. ${ }^{*} p<0.05,{ }^{* *} p<0.01,{ }^{* * *} p<0.001$, Mann Whitney test for (G-I). 


\subsection{Role of adiponectin in the clock-modulating effects of high fat diet}

Diet-induced obesity has been shown to down-regulate adiponectin (60). Feeding mice with a high fat diet (HFD) has been shown to disrupt circadian rhythms at both the behavioral and the molecular levels $(11,61)$. To investigate if adiponectin interacts with HFD-induced circadian modulations I compared circadian rhythms in WT and Adipoq KO mice fed with normal diet (ND) and HFD. HFD has been shown to acutely modulate diurnal feeding rhythms even before the development of obesity itself (11). Consistently, I also observed that HFD rapidly induced daytime feeding in WT mice compared to week 0 (i.e., fed with ND in all groups) (diet $x$ time variation, $F=9.949, p<0.0001$ ), but failed to do so in Adipoq KO mice which already showed an accentuated daytime feeding phenotype under ND conditions (diet $x$ time on diet, $F=0.2183$, p=0.9267) (Fig. 23 A). HFD did not significantly alter the total activity (Fig. 23 B) and daily energy intake (as compensated by reduced food intake) of both WT and Adipoq KO mice (Fig. 23 C \& D). Consistent with a previous report, HFD led to a higher body weight gain in Adipoq KO mice compared to WT despite their energy intake was comparable (Fig. 23 C), while on ND both groups showed no significant difference (Fig. 23 E) (45). Further, HFD lengthened the free-running period of locomotor activity in DD in WT and Adipoq KO mice, indicating that adiponectin plays no role in this effect (Fig. 23 F) (11). Finally, plasma adiponectin levels were significantly reduced in WT mice after 16 weeks of HFD (Fig. $23 \mathrm{H}$ ). These data suggest that the loss adiponectin is unlikely to be the cause of the HFD-induced modulation of circadian system. The absence of an effect of HFD on daytime feeding in Adipoq KO mice suggests that both HFD and adiponectin deficiency converge to the same mechanism to modulate diurnal feeding patterns which may potentially be mediated by altered regulation of the appetite-regulating NP circuitry in the MBH. 

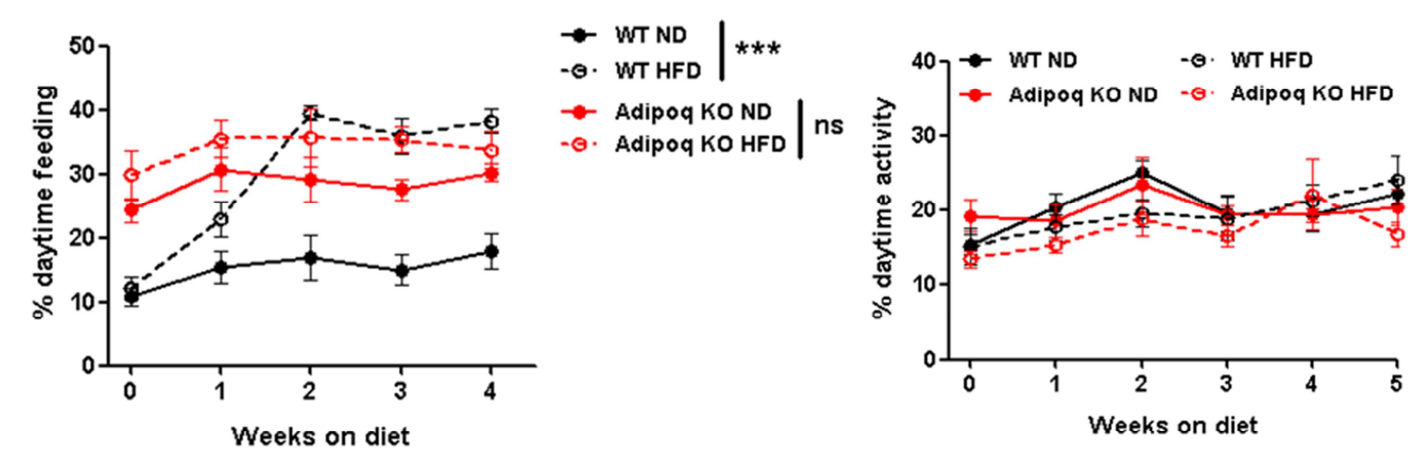

C

D

E
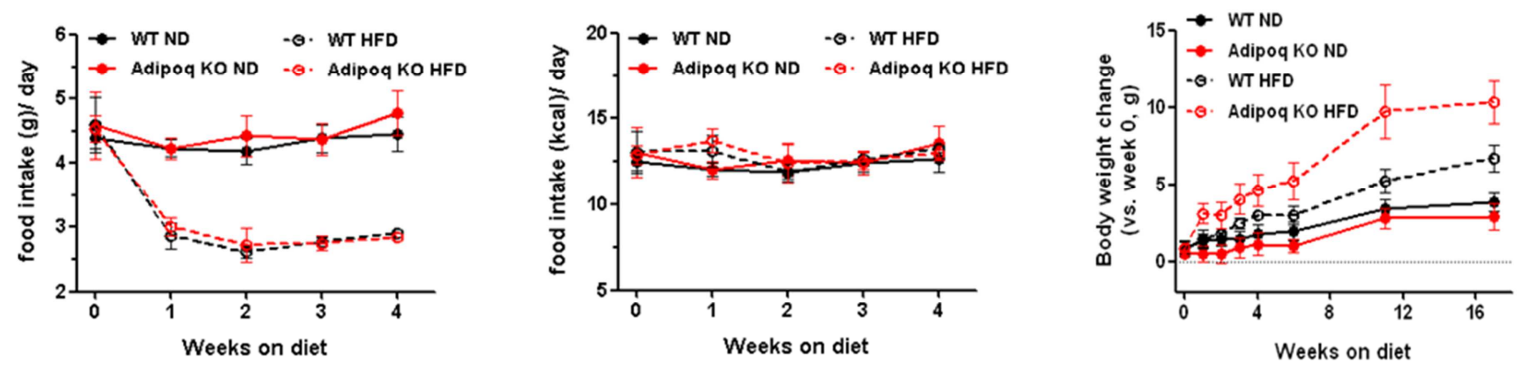

F
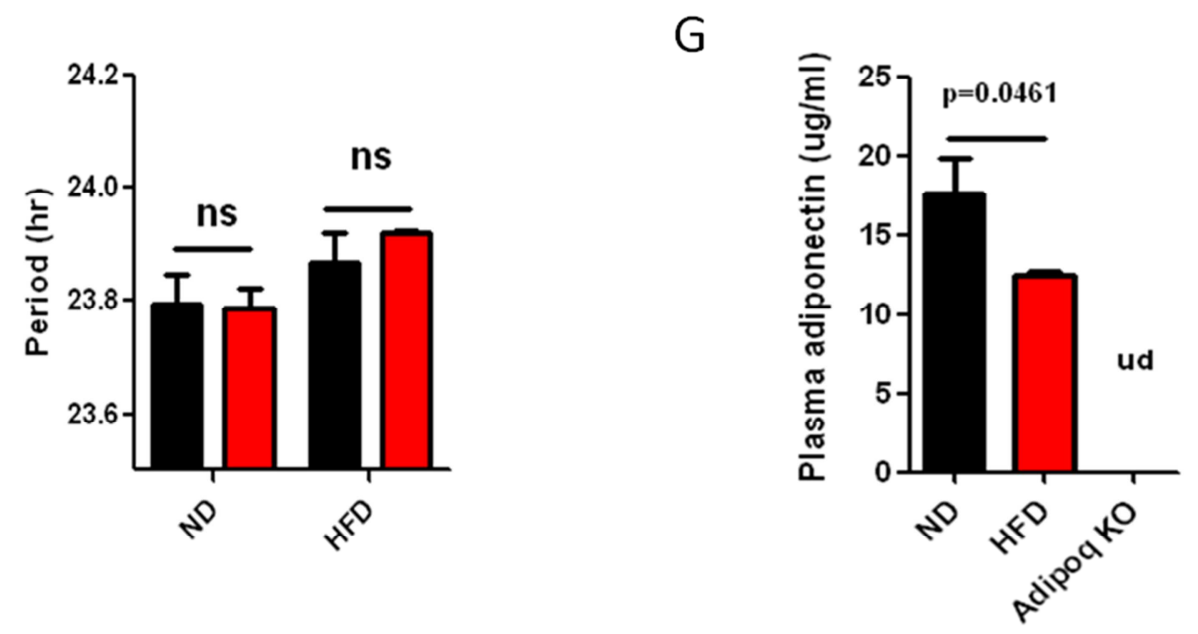

Figure 23. Role of adiponectin in the clock-modulating effects of high fat diet

(A) Normalized daily food intake during the light phase in WT and Adipoq KO mice fed with either normal diet (ND) or high fat diet (HFD) over 5 weeks of treatment. Week 0 refers to the week before HFD treatment in which all animals were fed with ND. The measurements were done once per week.

(B) Percentage of daily activity during the light phase.

(C and D) Food mass (C) and energy (D) intake per day.

(E) Body weight change since the start of experiment.

(F) Free-running period integrated from 10 days in DD on week 18-19 on diets. 
(G) Plasma adiponectin level in WT ND, HFD fed groups and Adipoq ND fed group after 20 weeks on diets measured at ZTO.

Error bars indicate means $\pm \mathrm{SEM}$, ( $\mathrm{n}=6$ per group throughout the experiment). Diet $\mathrm{x}$ time variation, ${ }^{* * *} \mathrm{p}<0.001$, Two-Way ANOVA in (A); ${ }^{*} p<0.05,{ }^{* * *} p<0.001, \mathrm{~ns}=$ not significant, ud= undetected, Mann-Whitney test in (F\&G).

\section{Discussion}

In this study, I identified adiponectin as a novel modulator of circadian clocks hypothalamic neurons of the $\mathrm{MBH}$. In vitro, I found that adiponectin treatment reset the $\mathrm{MBH}$ clock in a phase-dependent manner and induced Bmal1 upregulation. These effects were at least in part mediated via an AdipoR1-PGC1 $\alpha$ dependent pathway. Adipoq KO mice, while having a largely normal circadian activity rhythm, exhibited dampened feeding rhythms accompanied by dampened clock gene circadian expression rhythms and altered appetite-regulating NP diurnal expression profiles in the MBH. Under scheduled RF, Adipoq KO mice showed delayed development of FAA and an impaired FEO. In WT mice scheduled RF reinforced the diurnal oscillation of circulating adiponectin, upregulated the expression of adiponectin signaling components and clock gene oscillations in the $\mathrm{MBH}$ which may be involved in promoting the reorganization of diurnal expression of appetite-regulating NPs and, thus, the re-adaptation to the altered feeding rhythm. The importance of central adiponectin signaling for food anticipatory rhythm generation was further confirmed by i.c.v. administration of ROR $\alpha$ antagonists which phenocopied the Adipoq KO's FAA and food intake defects (Fig. 24). 
N44 Bmal1-Luc
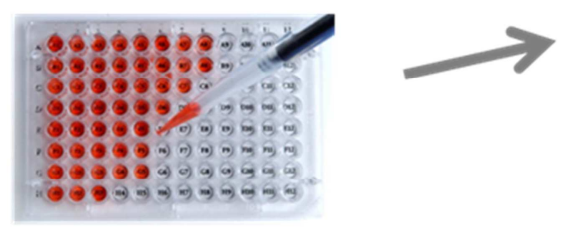

Adiponectin
AdipoR1-PGC1a

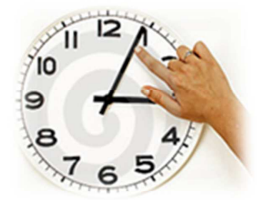

- Phase resetting

- Bmal1 Expression

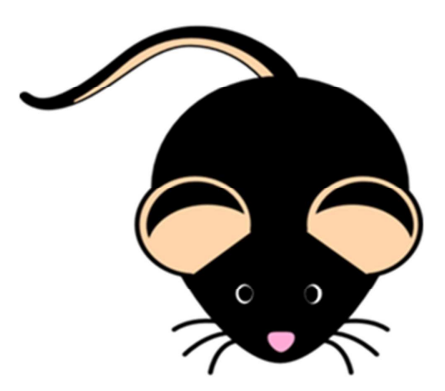

Adipoq KO
- Dampened diurnal feeding rhythm

- Altered hypothalamic clock genes \& appetite NP genes diurnal expression rhythm

- Altered food anticipitation under T-RF

- Adn $\rightarrow$ Adipor1 $\rightarrow$ PGC1a $\rightarrow$ Bmal1 pathway is involved in food anticipitory circadian rhythm under T-RF

\section{Figure 24. Summary of the findings in the current study}

In this study, I identified adiponectin as a novel circadian modulator of mediobasal hypothalamic neurons which can phase-reset the clocks and transcriptionally activate Bmal1 expression. These effects are at least in part mediated by an AdipoR1-PGC1 $\alpha$ dependent pathway. In vivo, I demonstrated that adiponectin deficient mice had dampened diurnal feeding rhythms and impaired food anticipatory circadian rhythms. These phenotypes were in line with the clock-modulating effects of adiponectin and the regulatory role of molecular clockwork on appetite-regulating neuropeptides expression.

\subsection{Molecular mechanism of the circadian effects of adiponectin}

I demonstrated in hypothalamic cells and in vivo that the AdipoR1-PGC1 $\alpha-B m a l 1$ axis plays a crucial role in the clock-modulating effects of adiponectin. However, it is highly surprising that while the components of this signaling pathway are believed to be ubiquitously expressed, I observed a strong tissue specificity of the circadian effects of adiponectin both in vitro and in vivo (Fig. 10 \& 15). One possibility is that the antagonistic effect of AdipoR2 on Bmal1 expression may counterbalance AdipoR1's effects in certain cell types. In N44 cells the loss of AdipoR2 led to a sharp downregulation of Nr1d1 which encodes for REV-ERB $\alpha$ - a 
transcriptional repressor of Bmal1 (Fig. 17 B). REV-ERB $\alpha$ inhibits Bmal1 transcription via binding to the RORE in Bmal1 promoter (62-64). It has been shown that REV-ERB $\alpha$ can antagonize the transactivating effect of PGC1 $\alpha$-ROR $\alpha$ complexes for Bmal1 induction (52). One potential mediator that links AdipoR2 to REV-ERB $\alpha$ is peroxisome proliferator-activated receptor alpha (PPAR $\alpha$ ). On one hand PPAR $\alpha$ has been shown to transcriptionally upregulate $\mathrm{Nr} 1 d 1$ expression (65), on the other hand AdipoR2 has been shown to activate PPAR $\alpha$ activity in the liver (66). The AdipoR2 effects on the circadian clock and its physiological relevance in different tissues remain to be further investigated. Another explanation may lie in the involvement of AMPK activation. It has been shown in peripheral tissues and fibroblasts that persistent activation of AMPK leads to an upregulation of CRY activity and subsequent dampening of the molecular clock due to increased negative feedback via REV-ERB $\alpha$ (56). In this study, I demonstrated that gAdn treatment led to an acute, but short-lasting induction of AMPK activation in hypothalamic neurons (Fig. 20). Activation kinetics of AMPK appear different in this context from what has been observed in peripheral tissues (66-68). Thus, it is possible that while acute AMPK activation may transiently activate PGC1 $\alpha$ activity and Bmal1 expression on one hand, extended AMPK activation in certain tissues may finally lead to a dampening of the circadian clock. Obviously, as adiponectin is known to activate multiple signaling pathways $(40,49)$, other potential mechanisms that may locally modify the adiponectin signaling to the molecular clock may exist depending on the cellular contexts which need to be further investigated.

\subsection{The role of adiponectin in photic entrainment of circadian rhythms}

The lack of overt abnormalities in the circadian activity rhythm of Adipoq KO mice suggests that the SCN pacemaker may not be a direct target of adiponectin. The accelerated re-adaptation after an experimental jetlag paradigm on the other hand implies that SCN input or downstream neural substrates may be subject to adiponectin's influences (33). Brain regions such as the intergeniculate leaflet (IGL) and raphe nucleus have been shown to modulate the function of the SCN and also express AdipoR1 (ISH data from Allen Brain Atlas). Therefore, it is possible that adiponectin may locally modify the physiological functions of these 
neural circuits. Further studies are needed to elucidate the mechanism of adiponectin's influences on the photic entrainment of circadian system.

\subsection{The role of adiponectin in feeding rhythm regulation}

The dampened feeding rhythms under both LD and DD conditions suggest that adiponectin plays a crucial role in regulating the circadian gating of appetite regulation (Fig. 9 A - F). In agreement with the in vitro data, the loss of adiponectin led to dampened clock gene rhythms in the $\mathrm{MBH}$ in vivo (Fig. $9 \mathrm{G}$ - I). Consistent with previous findings, I provided further evidence from Bmal1 $\mathrm{KO}$ mice and in vitro data to support that $\mathrm{MBH}$ clocks can directly regulate NP expression (Fig. 8 \& 11). However, owing to the complexity of the hypothalamic NP circuitry, the role of MBH clocks in specific subtypes of neurons in vivo needs to be further elucidated. From our data, I believe that the orexigenic NPs are more sensitive to adiponectin compared to anorexingenic NPs. It has been shown that central adiponectin applications acutely promote food intake (38). Therefore, the feeding phenotype observed in Adipoq KO mice may comprise of both clock-dependent and clock-independent pathways. In WT mice, I observed a significant diurnal oscillation of plasma adiponectin under ad libitum conditions which was further reinforced under RF conditions (Fig. 21 A). This diurnal oscillation appears to be heavily regulated by general metabolic state as under the scheduled RF protocol plasma adiponectin dramatically fell off after (re-)feeding (Fig. $21 \mathrm{~A}$ ), consistent with the described role of adiponectin as a hunger hormone (38). Under ad libitum feeding conditions, circulating adiponectin gradually increased in the second half of the night and reached its acrophase during the first half of the day. This is in phase with the MBH Bmal1 expression rhythm which peaks during the early morning (Fig. $9 \mathrm{G}$ ), suggesting that the late evening rise of circulating adiponectin may be important for the morning peak of MBH Bmal1 expression. This inference is further supported by the observation that i.c.v. administration of anti-adiponectin antibodies at late subjective night diminished Bmal1 expression in the $\mathrm{MBH}$ in the subjective morning (Fig. 13 L). 


\subsection{The role of adiponectin in food anticipation regulation}

In vitro, I observed that adiponectin could robustly phase-reset $\mathrm{MBH}$ neuronal clocks. The lack of obvious alterations of the phasing of the $\mathrm{MBH}$ clock gene rhythms in Adipoq $\mathrm{KO}$ mice under unchallenged conditions apparently suggests that adiponectin may not be a potent regulator of the phasing of the MBH clock when food is ubiquitously available. In contrast, the strong diurnal oscillation of plasma adiponectin under RF conditions indicates that adiponectin may become important in the regulation of physiological systems and behavioral neural circuits when food availability becomes scarce. The potential role of adiponectin in the kinetics of food entrainment of clock genes rhythms in non-hypothalamic tissues should be further investigated. At the same time, how adiponectin regulated $\mathrm{MBH}$ clock gene rhythms are translated into FAA and food intake behaviors is similarly worth studying (Fig. 12).

The ability to anticipate physiological needs in sync with predictable availability of valuable resources provides organisms evolutionary advantages. Light is the major Zeitgeber to the mammalian circadian system that entrains behavioral rhythms to the day-night succession. However, when the daily food access time window is shifted suddenly due to seasonal (i.e., photoperiod) change or other factors such as unexpected changes in ecological landscapes (e.g., wildfire), an anticipatory timekeeping mechanism that can reorganize and optimize the physiology for foraging according to the new food available time window may therefore increase the evolutionary success of an organism and is selected by evolution (15). In the current study, I provide strong evidence that central adiponectin signaling is involved in promoting the behavioral re-adaptation under RF. It has been shown previously that the appetite-regulating system plays a major role in the regulation of FAA. Peripheral metabolic hormones such as anorexigenic leptin and orexigenic ghrelin are an integral part of the food anticipatory system (19). Leptin deficient ob/ob mice and Zuker rats show increased FAA under RF which can be suppressed by administration of recombinant leptin $(69,70)$. In contrast, under RF ghrelin receptor deficient mice have attenuated FAA $(71,72)$, despite intriguingly that ghrelin deficient mice have been reported to have a normal FAA (73). More recently, as aforementioned, orexigenic AgRP/NPY neurons in the ARC have been suggested as crucial elements of the food anticipation circuitry (29). These observations strongly point out that the interaction of metabolic 
hormones and $\mathrm{MBH}$, particularly the orexigenic neural circuits, is a key regulator of the food anticipatory system. Adipoq KO mice displayed a delayed development of FAA and RF-mediated entrainment of food intake. Critically, these phenotypes were mimicked by central administration of a ROR $\alpha$ antagonist (Fig. 22), which excluded the potential secondary off-target peripheral influences downstream to the loss of adiponectin in Adipoq KO mice such as alteration of immune function or lipid homeostasis (37). In the i.c.v. VPR66 administration experiment, I noticed that VPR66 did not acutely inhibit FAA and food intake on the day of injection, but did do so with a day of delay, suggesting that it does not act directly, but by incorporation of external signals into MBH clock feedback loops. This also argues against the involvement of an acute hypothalamic orexigenic effect of adiponectin mediated via AMPK signaling (38). Rather VPR66 may antagonize the adiponectin-mediated resetting of clock gene expression rhythms in the MBH which mediates adaptation of appetite systems and behavior to the RF schedule through reprogramming of diurnal NP expression. Multiple lines of evidence support this interpretation. First, as mentioned, the orexigenic neuronal network in the MBH has been shown to be important for FAA circadian rhythms (46). Second, RF has been shown to reinforced and phase-reset the clock gene oscillations in the ARC and DMH $(74,75)$. Finally, extra-SCN neural Bmal1 expression has been shown to contribute to the robustness of the FEO (48). These data are in line with our observation that adiponectin acts as a positive regulator of Bmal1 expression and that the CLOCK/BMAL1 dimer is an important regulator of appetite-regulating NP expression (76). However, further studies with genetically and anatomically defined abrogation of central adiponectin signaling in specific neuronal subtypes will be needed to identify the responsible neural circuitries involved in the regulation of adiponectin-regulated FAA rhythms. 


\subsection{The role of adiponectin in high fat diet induced circadian disturbances}

HFD has been shown to promote daytime feeding independent of the development of obesity (11). In this study, I also observed similar effects of HFD in WT animals, but failed to further induce daytime feeding in Adipoq KO mice (Fig. 23 A). One explanation may be a convergence of the physiological routes of adiponectin deficiency and HFD to induce daytime feeding (i.e. ceiling effect in Adipoq KO mice) - which may likely occur at the level of appetite regulating hypothalamic NPs - HFD treatment dramatically suppresses the orexigenic AgRP and Npy overall diurnal variations but upregulated the overall diurnal expression of anorexigenic Cart and Pomc (11). In our hands WT mice fed with HFD did not reach the morbid obese state in which the circulating adiponectin dramatically has been reported to drop to less than one fifth of ND fed controls according to some other papers (60), thus precluding assessment of the potential effects of the pathophysiological loss of adiponectin induced by morbid obesity in the circadian functions (i.e., in contrast to the complete prenatal loss of adiponectin in Adipoq KO mice). Under basal conditions, Adipoq mice have been reported to have only minor, if any, effects on various metabolic parameters $(44,45)$. However, under HFD challenge, Adipoq KO mice show significant reduction in energy expenditure, impairment of systemic glucose and lipid homeostasis and obesity compared to wild-type control animals (45). In line with this previous report, I observed that Adipoq KO mice in our hand were also susceptible to HFD-induced weight gain despite of comparable energy intake. This suggests an altered energy turnover in Adipoq KO mice under a HFD challenge. Mistimed high fat feeding during the normal rest phase has been shown to promote energy incorporation and bodyweight gain (12). Given that Adipoq KO mice also showed an increased daytime feeding phenotype, it is tempting to postulate that a part of the body weight gain may be due to mistimed feeding. However, further experiments with carefully designed feeding time windows are clearly needed to dissect the relative contribution of metabolic functions of adiponectin and feeding rhythm disruption to the body weight gain in Adipoq KO mice upon HFD challenge.

While this thesis project was under way adiponectin has been independently reported as a circadian clock modulator in peripheral organs (77). However, owing to the different approaches chosen in that study compared to ours (ectopic overexpression of adiponectin, use of heterogenic mouse models and 
physiological systems of interest), a direct comparison between both studies proves difficult. Nevertheless, Hashinaga T. et al also came to the conclusion that adiponectin signaling may play an important role in maintaining proper circadian rhythms of clock gene expression in certain physiological systems under metabolic challenge (77). They reasoned that a part of the circadian effects of adiponectin may be mediated by suppressing the expression of a pro-inflammatory cytokine - tumor necrosis factor alpha (TNF $\alpha$ ) which has been shown to modulate the circadian clock system upon inflammatory challenges (78). More recently, the expression of adiponectin in adipose tissues has been shown to be subject to molecular clock regulation via PGC1a and PPARy (79), thus further strengthening the claim that adiponectin is an integral part of the circadian metabolic circuitry.

\subsection{Clinical implications}

Together, the present study reveals that adiponectin is a metabolic feedback signal to the mediobasal hypothalamic circadian clock where it regulates feeding rhythms. Disturbance of adiponectin signaling such as in obesity may therefore involve in alter feeding rhythms as seen in patients suffering from night eating syndrome (NES). NES is characterized by a lack of appetite in the morning, but consuming a half or more of daily food intake in the evening and at night associated with various sleep abnormalities (80). Obesity has a strong positive correlation with NES (81). NES patients have increased hunger at night accompanied with blunted nocturnal rise of leptin (82) and a phase advance of ghrelin (83). Mistimed intake of high energy food, on the other hand, has been shown to further promote metabolic disorders, thus forming a viscous cycle $(4,12,13)$. The positive role of adiponectin in FAA suggests that people suffering from adiponectin deficiency might have impairment in adapting their feeding rhythms when food availability time window is changed. Though direct evidence that link metabolic syndromes and appetite regulation after a shift of Zeitgeber cycle is still missing, a recent epidemiological study supports this inference showing that that body mass index (BMI) has a positive correlation with social jetlag though the blood hormones has not been investigated in this study (84). Occupations that require frequent shiftwork may represent a condition when food intake becomes uncoupled from the SCN. It can be expected that people with adiponectin deficiency 
will suffer from more severe loss of appetite following the shift and require more time to recover under such conflicting Zeitgeber conditions. Thus, together with other metabolic humoral factors reported in literature, I conclude that proper metabolic feedback signals to the circadian clock system either directly or indirectly are important for the robustness of the circadian clock and therefore contribute to the general well-being (Fig. 25).

Our study also sheds light on the therapeutic potential of adiponectin signaling in inhibiting circadian disruption associated with metabolic disorders. Recently, a small molecule agonist of AdipoRs (for both R1 and R2) has been developed, thus providing an opportunity to test this idea in preclinical animal models of circadian disruption (85). Timed administration of adiponectin or AdipoRs' agonists may help to normalize the altered circadian appetite rhythms in individuals suffering from obesity or NES and circadian misalignments such as shiftworkers. Development of individual receptor-specific agonists/antagonists on the other hand will be helpful to further delineate the molecular mechanism of the circadian effects of adiponectin signaling.

\subsection{Concluding remarks}

This study identified adiponectin as a novel modulator of the $\mathrm{MBH}$ clocks and is involved in regulating feeding rhythms and food anticipatory circadian rhythms which at least partly mediated by a mechanism dependent of the AdipoR1-PGC1 $\alpha /$ ROR $\alpha$-Bmal1 pathway. 


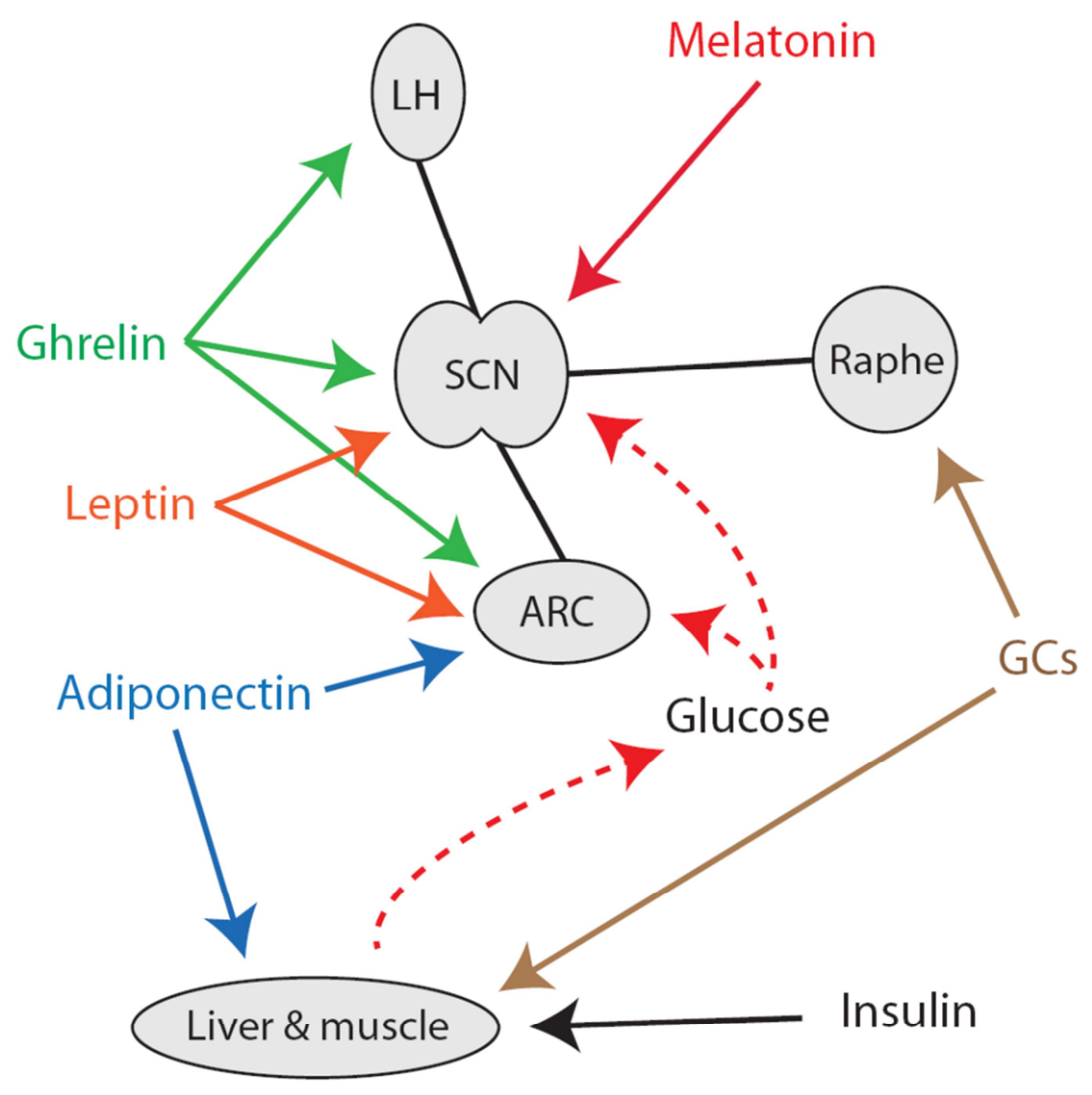

Figure 25. Overview of established endocrine feedback to the circadian clock.

While the circadian system are known to regulate the diurnal hormone abundance in the bloodstream, a number of metabolic peptide hormones such as insulin, leptin and ghrelin have been shown to modify the molecular rhythms directly on central and peripheral clock function. In the CNS, leptin and ghrelin have been shown to directly modulate the SCN clock and may also presumably affect the circadian clock in mediobasal hypothalamic structures such as arcuate nucleus (ARC) or lateral hypothalamus (LH). In this study, I identify adiponectin as a new player to modulate the MBH's clocks and may also impinge on other central and peripheral clocks as well. Melatonin and glucocorticoid (GC) are two well established feedback hormones that modify the circadian clocks of a board spectrum of central and peripheral tissues. Their productions are strongly influenced by the circadian clock. While melatonin can directly act on the SCN, the SCN is insensitive to GC. However, GC can also modulate the SCN clock indirectly via other brain structures such as the raphe nuclei of the brainstem. The central clocks may also be affected by metabolic hormones indirectly via changes in blood metabolite levels such as glucose or free fatty acid. Adapted from Ref. 9. 


\section{Materials and Methods}

Unless otherwise stated, standard chemicals were purchased from Roth (Karlsruhe, Germany), tissue culture products were obtained from Gibco (Life Technologies GmbH, Darmstadt, Germany).

\subsection{Animals and circadian behavioral experiments}

Adiponectin knockout mice (JAX stock \#008195) and PER2::LUCIFERASE animals (Per2-luc; JAX stock \#006852) were purchased from the Jackson's Laboratory (Maine, USA) and maintained at the animal facilities in Göttingen or Lübeck. The use of Bmal1 KO mice has been described previously (86). All mice used were kept on C57BL6J genetic background. For all experiments, unless stated otherwise, male mice were individually housed under 12-hour light, 12-hour dark conditions (LD; 100 lux) with ad libitum access to chow pellets (normal diet (3.4\% kJ fat, Ssniff \#V1536) or high fat diet (45\% kJ fat, Ssniff \#EF D12451) and water. For experiments in constant darkness (DD), mice were first entrained under LD conditions for at least a week and then released into DD for the indicated timespans. Behavioral experiments were performed on animals aged 10-12 weeks at the beginning of the experiment. Molecular analyses were performed on 16-24 weeks old animals. All animal experiments (including MEF cells and primary hypothalamic neuron isolations) were done after ethical assessment and licensed by the Office of Consumer Protection and Food Safety of the State of Lower Saxony and the Ministry of Agriculture of the State of Schleswig-Holstein and in accordance with the German Law of Animal Welfare (TierSchG).

\subsection{Plasmid construction}

pLKO-WPRE-GFP was modified from the original pLKO.1-TRC (Addgene plasmid \#10878) backbone in which the puromycin resistance open reading frame (ORF) was replaced with a GFP-WPRE sequence from pLenti-CMV-GFP-Zeo (637-7) (Addgene plasmid \#17449) with Kpn I and BamH I restriction sites. Nucleotide sequences for generating shRNAs are listed in the Table 3 and were cloned into pLKO-WPRE-GFP using Age I and EcoR I restriction sites. 
Npy-luc plasmid was generated by cloning a $\sim 2 \mathrm{~kb}$ 5'-regulatory fragment of the murine Npy gene into pGL4 vector (Promega, Mannheim, Germany) with Kpn I and BgI II restriction sites. This fragment has previously been identified to contain multiple E-boxes (22). The integrity of the recombinant plasmids was confirmed by sequencing.

\subsection{Lentivirus production and transduction}

The Bmal1-luc encoding pBluF-puro plasmid was a kind gift from Prof. Steven Brown, University of Zurich, Switzerland. To produce Bmal1-luc lentiviral particles, a 10-cm dish of HEK293T cells was cotransfected with

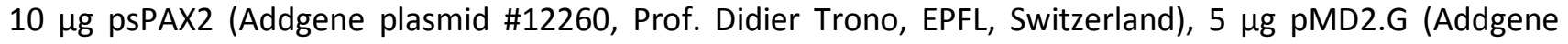
plasmid \#12259, Prof. Didier Trono, EPFL, Switzerland), and $15 \mu \mathrm{g}$ pBluF-puro using Xfect transfection reagent (Clontech, Saint-Germain-en-Laye, France). Cells were rinsed with PBS on the second day and restored with fresh culture medium. Viral containing medium was harvested at $36 \mathrm{hr}$ after transfection and stored at $4{ }^{\circ} \mathrm{C}$. Cells were restored with fresh medium, and further medium collections were performed on the next day. Two collections were pooled and concentrated using LentiX concentrator reagent (Clontech) according to the manufacturer's protocol. Virus titers were determined by transducing HEK293T cells with a serial dilution of GFP encoding lentiviral particles (produced as described above but with pWPI (Addgene plasmid \#12254, Prof. Didier Trono, EPFL, Switzerland) instead of pBluF-puro). At $72 \mathrm{hr}$ after transduction, GFP-positive cells were counted under a fluorescence microscope. The same serial dilution was also subjected to quantitative real-time PCR (qPCR) analysis using a primer pair amplifying the viral WPRE element (forward: 5'-GGCACTGACAATTCCGTGGT-3'; reverse: 5'-AGGGACGTAGCAGAAGGACG-3') to determine viral genome abundance. $\mathrm{qPCR}$ was also done for Bmal1-luc virus containing medium, and the titer was estimated by comparison with pWPI abundance in relation to the GFP calibration curve. To transduce hypothalamic cells, cells at $50 \%$ confluence were loaded with $\sim 0.85 \times 10^{8}$ infection units (IFUs) per $1 \mathrm{ml}$ medium in the presence of $8 \mu \mathrm{g} / \mathrm{mL}$ polybrene. Experiments on cells were carried out $72 \mathrm{hr}$ after transduction. 


\subsection{Cell culture and circadian luminescence recording}

mHypoE-N44, N41 (Cedarlanelabs, NC, USA), HEK293T, NIH3T3 (ATCC, LGC Standards GmbH, Wessel, Germany), and MEF cells were maintained in DMEM with $2 \mathrm{mM}$ glutamine supplemented with $10 \%$ fetal bovine serum (FBS) and $1 \%$ penicillin/streptomycin at $37{ }^{\circ} \mathrm{C}$ with $5 \% \mathrm{CO}_{2}$. Cells stably expressing Bmal1-luc reporter via lentiviral transduction were generated polyclonally with puromycin selection. For circadian luminescence measurements, cells seeded in 96-well plates were synchronized by $100 \mathrm{nM}$ dexamethasone treatment for 2 hours. After that, medium was replaced with recording medium (DMEM without phenol red supplemented with $2 \mathrm{mM}$ Glutamax, $3 \mathrm{mM}$ sodium carbonate, $10 \mathrm{mM}$ HEPES, $2 \%$ B-27 supplement, $1 \mathrm{x}$ penicillin/streptomycin and $0.5 \mathrm{mM}$ D-luciferin). The plates were then sealed with transparent films and luminescence was recorded at $34{ }^{\circ} \mathrm{C}$ using the Berthold TriStar LB 941 plate reader (Berthold Technologies, Wildbach, Germany).

\subsection{Primary hypothalamic neuronal culture}

Hypothalami of E16 embryos were dissected and isolated using the papain dissociation system (Worthington) according to the manufacturer's protocol. $3.25 \times 10^{5} / \mathrm{cm}^{2}$ viable cells in plating medium (neural basal medium supplemented with 2 mM Glutamax, 2 \% B-27, $10 \%$ FBS and $1 \%$ pen/strep) were seeded to vessels double-coated with poly-D-lysine and laminin. On the next day, the plating medium was replaced with feeding medium (same as plating medium, but without FBS) and transduced with Bmal1-luc lentivirus. $24 \mathrm{hr}$ later, half the volume of the old medium was refreshed with fresh feeding medium containing $5 \mu \mathrm{M}$ cytosine arabinoside. Half the volume of the old medium was subsequently refreshed every 3 days. On day in vitro (DIV) 9, neurons were subjected to bioluminescence experiments in feeding medium supplemented with D-luciferin. 


\subsection{ARC/ME slice cultures}

Luminescence was measured from cultured ARC/ME slices of heterozygous Per2-luc mice as described previously (21). Briefly, brains were isolated and harvested in ice-cold Hank's balanced salt solution (HBSS). $300 \mu \mathrm{m}$ thick $\mathrm{ARC} / \mathrm{ME}$ coronal slices $(-1.80 \mathrm{~mm}$ and $-2.10 \mathrm{~mm}$ relative to bregma) were prepared using a vibratome (Thermo Scientific, MA, USA). The slices were immediately placed onto a culture plate insert (Merck Millipore, Darmstadt, Germany) in 35-mm petri dishes filled with $1 \mathrm{ml}$ recording medium (same as the one used for cell culture). Luminescence was measured in a LumiCycle (Actimetrics Inc, IL, USA) at 32.5 ${ }^{\circ} \mathrm{C}$.

\subsection{Quantitative real-time polymerase chain reaction}

Isolated tissues were harvested and kept in RNAlater solution (Ambion, Life Technologies $\mathrm{GmbH}$ ) according to the manufacturer's protocol. Total RNA of tissues and cell cultures was extracted using TRIzol reagent (Invitrogen, Life Technologies $\mathrm{GmbH}$ ). cDNA synthesis was performed using the High Capacity cDNA Reverse Transcription Kit (Life Technologies) with random hexamer primers. qPCR was performed using GoTaq qPCR Master Mix (Promega GmbH, Mannheim, Germany) on a CFX96 thermocycler (Bio-Rad, Munich, Germany). Relative gene expression was quantified using a $\Delta \Delta$ threshold cycle $(\mathrm{Ct})$ method with adjustments of the amplification efficiencies of individual primer pairs; Ef1 $\alpha$ was used as the reference gene (except for the epididymal fat pads for which B-actin was used as reference). Primer sequences are listed in Table 3.

\subsection{Western blot}

To detect BMAL1 protein in N44 cells, cells were lysed in a lysis buffer (1 \% triton X-100, $2 \%$ sodium dodecyl sulfate (SDS), $1 \%$ sodium deoxycholate, $1 \%$ NP40 and $1 x$ cOmplete protease inhibitor cocktail (Roche, Grenzach-Wyhlen, Germany) in Tris-buffer saline (TBS) and subjected to sonication with the Branson 450 sonifier (Thermo Scientific; amplitude: 50, duty: 30\%, duration: $30 \mathrm{sec}$ ) and subsequent Western blot analysis according to standard protocols of SDS-polyacrylamide gel electrophoresis (PAGE) and Western blot (87) using anti-BMAL1 antibody (dilution 1:1000; Novus Biologicals, Cambridge, UK). To detect the 
phosphorylation of the AMPK alpha subunit, N44 cells were harvested in a lysis buffer with phosphatase inhibitors ( $1 \%$ triton X-100, 2 \% SDS, $1 \%$ sodium deoxycholate, $1 \%$ NP40 and $1 \mathrm{x}$ cOmplete protease inhibitor cocktail, $1 \mathrm{mM}$ dithiothreitol (DTT), $50 \mathrm{mM}$ sodium fluoride ( $\mathrm{NaF}$ ), $1 \mathrm{mM}$ sodium orthovandate $\left(\mathrm{NaVO}_{5}\right), 5 \mathrm{mM}$ sodium pyrophosphate $\left(\mathrm{NaPP}_{\mathrm{i}}\right)$ in $\left.\mathrm{TBS}\right)$ and subjected to sonication and subsequent Western blot analysis with anti-phospho-AMPK $\alpha$ (Thr172) (dilution 1:1000) and anti-AMPK $\alpha$ (dilution 1:1000) antibodies (Cell Signaling Technology, MA, USA). To detect PGC1 $\alpha$ in the MBH, tissues were dissected and frozen immediately on dry ice and stored at $-80^{\circ} \mathrm{C}$ until use. To extract protein, the MBH was homologized in RIPA buffer (150 mM sodium chloride $\left(\mathrm{NaCl}_{2}\right), 10 \mathrm{mM}$ Tris, $0.1 \%$ SDS, $0.1 \%$ triton X-100, 25 $\mathrm{mM}$ sodium deoxycholate, $5 \mathrm{mM}$ ethylenediaminetetraacetic acid (EDTA) and sodium deoxycholate) and boiled for 5 minutes. The lysates were then subjected to Western blot analysis using anti-PGC1 $\alpha$ antibody (H300) (dilution 1:500; Santa Cruz Biotechnology, Heidelberg, Germany). In all cases, the protein concentration was determined with BCA protein assay kit (Thermo Scientific); Endogenous $\beta$-tubulin level determined with anti- $\beta$-tubulin (dilution 1:1000; Cell Signaling Technology) was used as loading reference. Densitometry analyses of bands intensity were performed with Quantity One software (Bio-Rad).

\subsection{Chromatin immunoprecipitation}

$2 \mathrm{hr}$ after adiponectin treatment, N44 cells were fixed with $1 \%$ formaldehyde at $37{ }^{\circ} \mathrm{C}$ for 10 minutes. Cells were then lysed with SDS lysis buffer (1 \% SDS, 19 mM EDTA, 50 mM EDTA, 50 mM Tris, pH8.1 with 1x cOmplete protease inhibitor cocktail) and subjected to sonication using Branson 450 sonicator (amplitude: 50, duty: 30\%, duration: $30 \mathrm{sec}, 6$ repeats with 1 min intervals between each sonication) which yielded a majority of DNA fragments with sizes of 200-1000 bp. Precleared samples were incubated overnight at $4{ }^{\circ} \mathrm{C}$ with $10 \mu \mathrm{g}$ anti-PGC1 $\alpha$ antibody (H300). The samples were incubated with Protein-G agarose beads (Thermo Scientific) for $1 \mathrm{~h}$ at $4{ }^{\circ} \mathrm{C}$ followed by intensive washings. Afterward, immune complexes were eluted with elution buffer ( $1 \%$ SDS, $0.1 \mathrm{M}$ sodium hydrogen carbonate $\left(\mathrm{NaHCO}_{3}\right)$. The eluates were then treated with 40 $\mu \mathrm{g} / \mathrm{ml}$ proteinase $\mathrm{K}$ and further subjected to phenol-chloroform-isoamyl alcohol purification. Purified 
samples were then subjected to qPCR analysis with primer pairs flanking the RORE and the $3^{\prime}$-UTR of Bmal1 and values were normalized to the pre-IP inputs. Primer sequences are listed in Table 3.

\subsection{NPY promoter end-point luciferase assays}

HEK293T cells were plated into 96-well plates coated with poly-D-lysine and transfected with the following expression plasmids: $10 \mathrm{ng} N P Y$-luc and $2 \mathrm{ng} C M V$-Renilla luciferase in various combinations of the following clock gene constructs: HA-Clock, HA-Bmal1, CRY1 and pcDNA3.1 (mock transfection) using Lipofectamine LTX transfection reagent (Life Technologies). $48 \mathrm{hr}$ after transfection, luciferase activity was measured using Dual-Glo Luciferase Assay System (Promega) and the Berthold TriStar plate reader.

\subsection{Intracerebroventricular administration}

Intracerebroventricular (i.c.v.) cannulae were implanted to mice at 8-10 weeks of age under isoflurane anesthesia (CP-Pharma, Burgdorf, Germany) and carprofen analgesia (5 mg/kg; Rimadyl, Pfizer Deutschland $\mathrm{GmbH}$, Berlin, Germany). A stainless steel guide cannula (internal cannula $2.8 \mathrm{~mm}$ with $1 \mathrm{~mm}$ projection 33 gauge, C315I-5/Spc; Plastics One, VA, USA) was stereotaxically implanted into the lateral cerebral ventricle (0.9 lateral and 0.1 posterior to bregma, 2.2 ventral to the surface of the skull). The cannula was fixed with dental cement on two small steel screws (screw 00-96x1/96). After surgery animals were single-caged and allowed to recover for 1-2 weeks with handling everyday. Substances for i.c.v. administration were dissolved in artificial cerebrospinal fluid (aCSF; $125 \mathrm{mM} \mathrm{NaCl}, 25 \mathrm{mM} \mathrm{NaHCO}_{3}, 1.25 \mathrm{mM}$ sodium phosphate monobasic monohydrate $\left(\mathrm{NaH}_{2} \mathrm{PO}_{4}\right), 20 \mathrm{mM}$ D-glucose, $5 \mathrm{mM}$ potassium chloride $(\mathrm{KCl}) .2 \mu \mathrm{l}$ in total volume were injected into manually restrained awake mice. 


\subsection{ELISA analysis of plasma adiponectin}

Blood was collected from decapitated animals into EDTA-containing tubes. Plasma concentrations of adiponectin were determined with an ELISA detection kit for mouse adiponectin (Adipogen Inc, CA, USA) according to the manufacturer's protocol using the Epoch microplate spectrophotometer (Biotek Instruments, VT, USA).

\subsection{Statistical analysis}

To access the circadian parameters of luminescence recording rhythms, all traces were subjected to the subtraction of their own 24-hr moving average as baselines. To construct phase response curves, the baseline-subtracted data were further fitted with a standard sine wave regression. The intersection of the ascending cross-section of the sine wave with the $x$-axis was defined as 0 or $2 \pi$ in radian and the peak as 0.5

$\pi$. To compare the phase before and after drug treatments, phase shifts were determined by comparing extrapolated peak times from sine wave fits of at least two consecutive circadian cycles before and after the treatment. All measurements of circadian parameters were performed using the Lumicycle analysis software (Actimetrics). Diurnal variation of $24-\mathrm{hr}$ profile data was tested using sine-cosine fitting with CircWave software version 3.3 (University of Groningen, NL). General statistical analysis was performed using GraphPad Prism 5.0 (GraphPad, CA, USA). All data are represented as means \pm standard error mean (SEM). In general, Student's t test was used for pairwise comparison in cell-based experiments and Mann Withney test for pairwise comparison in animal experiments. Multi-groups analyses were performed with One-Way ANOVA with either Tukey or Bonferroni post-test. 24-hr profile experiments were analyses with Two-Way ANOVA with Bonferroni post-test for comparison at specific time point. 
Table 3. Sequence information of the oligos used in current study.

\begin{tabular}{|c|c|c|c|}
\hline Target & $\begin{array}{c}\text { shRNA Sequences } \\
\text { Sense }\end{array}$ & & Reference/Source \\
\hline scramble & CCTAAGGTTAAGTCGCCCTCG & & Addgene plasmid 8455 \\
\hline AdipoR1 KD1 & GTACGTCCAGGCTTCAAATAA & & TRCN0000249147 \\
\hline AdipoR1 KD2 & AGATGGAGGAGTTCGTGTATA & & TRCN0000257836 \\
\hline AdipoR2 KD1 & GCAGGAATTTCGTTTCATGAT & & TRCN0000175771 \\
\hline AdipoR2 KD2 & CTTCTGATGTGATCGTATTIA & & TRCN0000277168 \\
\hline Bmal1 KD1 & GACGAACTGAAACACCTAATT & & TRCN0000282305 \\
\hline PGC1 $\alpha$ KD & TAACTATGCAGACCTAGATAC & & TRCN0000219080, (ref) \\
\hline Target & $\begin{array}{c}\text { qPCR } \\
\text { Forward } \\
\end{array}$ & Reverse & Reference/Source \\
\hline Eef1 $\alpha$ & CACATCCCAGGCTGACTGT & TCGGTGGAATCCATTTGTT & \\
\hline Bmal1 & TGACCCTCATGGAAGGTTAGAA & CAGCCATCCTTAGCACGGT & \\
\hline Per2 & GCCAAGTTTGTGGAGTTCCTG & CTTGCACCTTGACCAGGTAGG & \\
\hline$D b p$ & AATGACCTTTGAACCTGATCCCGCT & GCTCCAGTACTTCTCATCCTTCTGT & \\
\hline Nr1d1 & AGCTCAACTCCCTGGCACTTAC & CTTCTCGGAATGCATGTTGTTC & \\
\hline$N P Y$ & CTCCGCTCTGCGACACTAC & GGAAGGGTCTTCAAGCCTTGT & \\
\hline$A G R P$ & ATGCTGACTGCAATGTTGCTG & CAGACTTAGACCTGGGAACTCT & \\
\hline CART & CCCGAGCCCTGGACATCT & GCTTCGATCTGCAACATAGCG & \\
\hline POMC & ATGCCGAGATTCTGCTACAGT & TCCAGCGAGAGGTCGAGTTा & \\
\hline HCRT & GTCGCCAGAAGACGTGTTC & GGTGGTAGTTACGGTCGGAC & \\
\hline AdipoR1 & AATGGGGCTCCTTCTGGTAAC & GCAGACCTTATACACGAACTCC & \\
\hline AdipoR2 & CCTITCGGGCCTGTTITAAGA & GAGTGGCAGTACACCGTGTG & \\
\hline Adipoq & GCAGGCATCCCAGGACATC & GCGATACACATAAGCGGCTTCT & \\
\hline$P G C 1 \alpha$ & AACCAGTACAACAATGAGCCTG & AATGAGGGCAATCCGTCTTCA & \\
\hline $\operatorname{cdh} 13$ & ATGGCAGAACTCGTGATTGTC & CGCTATCGACTACCTTGCCG & \\
\hline B-actin & GATGACCCAGATCATGTTTGAG & GCTGTGGTGGTGAAGCTGTA & \\
\hline Target & $\begin{array}{c}\text { CHIP-qPCR } \\
\text { Forward } \\
\end{array}$ & Reverse & \\
\hline Bmal1 RORE & TTGGGCACAGCGATTGGTGG & TCCGGCGCGGGTAAACAGG & \\
\hline Bmal1 3'UTR & CTGTGTGCATCGGACAGTC & CGAAGCCACCATCTGAAAC & \\
\hline Target & Cloning & & Reference/Source \\
\hline NPY promoter & GTCCAGGAGGTGATGAACCTATGTTCTITATGG & GCGCCCCTGTCCCAGTTGATCCTGGC & \\
\hline EGFP-WPRE & ATGGTGAGCAAGGGCG & CAGGCGGGGAGGCGGC & Addgene plasmid 17449 \\
\hline
\end{tabular}

\section{References}

1. Pan A, Schernhammer ES, Sun $Q$, Hu FB. Rotating night shift work and risk of type 2 diabetes: two prospective cohort studies in women. PLoS Med 8, e1001141 (2011).

2. Marqueze EC, Ulhoa MA, Moreno CR. Irregular working times and metabolic disorders among truck drivers: a review. Work 41 Suppl 1, 3718-25 (2012). 
3. Garaulet M, Ordovas JM, Madrid JA. The chronobiology, etiology and pathophysiology of obesity. Int J Obes (Lond) 34, 1667-83 (2010).

4. Garaulet M, Madrid JA. Chronobiological aspects of nutrition, metabolic syndrome and obesity. Adv Drug Deliv Rev 62, 967-78 (2010).

5. Dibner $\mathrm{C}$, Schibler $\mathrm{U}$, Albrecht $\mathrm{U}$. The mammalian circadian timing system: organization and coordination of central and peripheral clocks. Annu Rev Physiol 72, 517-49 (2010).

6. Huang W, Ramsey KM, Marcheva B, Bass J. Circadian rhythms, sleep, and metabolism. J Clin Invest 121, 2133-41 (2011).

7. Asher G, Schibler U. Crosstalk between components of circadian and metabolic cycles in mammals. Cell Metab 13, 125-37 (2011).

8. Zanquetta MM, Correa-Giannella ML, Monteiro MB, Villares SM. Body weight, metabolism and clock genes. Diabetol Metab Syndr 2, 53 (2010).

9. Tsang AH, Barclay JL, Oster H. Interactions between endocrine and circadian systems. J Mol Endocrinol 52, R1-16 (2014).

10. Damiola F, Le Minh N, Preitner N, Kornmann Bt, Fleury-Olela F, Schibler U. Restricted feeding uncouples circadian oscillators in peripheral tissues from the central pacemaker in the suprachiasmatic nucleus. Genes \& Development 14, 2950-61 (2000).

11. Kohsaka A, Laposky AD, Ramsey KM, Estrada C, Joshu C, et al. High-fat diet disrupts behavioral and molecular circadian rhythms in mice. Cell Metab 6, 414-21 (2007).

12. Hatori M, Vollmers C, Zarrinpar A, DiTacchio L, Bushong EA, et al. Time-restricted feeding without reducing caloric intake prevents metabolic diseases in mice fed a high-fat diet. Cell Metab 15, 848-60 (2012).

13. Mattson MP, Allison DB, Fontana L, Harvie M, Longo VD, et al. Meal frequency and timing in health and disease. Proc Natl Acad Sci U S A 111, 16647-53 (2014).

14. Mistlberger RE. Food-anticipatory circadian rhythms: concepts and methods. Eur J Neurosci 30, 1718-29 (2009). 
15. Antle MC, Silver R. Neural basis of timing and anticipatory behaviors. Eur J Neurosci 30, 1643-9 (2009).

16. Honma K, Honma S. The SCN-independent clocks, methamphetamine and food restriction. Eur J Neurosci 30, 1707-17 (2009).

17. Davidson AJ. Lesion studies targeting food-anticipatory activity. Eur J Neurosci 30, 1658-64 (2009).

18. Challet E, Mendoza J, Dardente H, Pevet P. Neurogenetics of food anticipation. Eur J Neurosci 30, 1676-87 (2009).

19. Escobar C, Cailotto C, Angeles-Castellanos M, Delgado RS, Buijs RM. Peripheral oscillators: the driving force for food-anticipatory activity. Eur J Neurosci 30, 1665-75 (2009).

20. Adamantidis A, de Lecea L. Sleep and metabolism: shared circuits, new connections. Trends Endocrinol Metab 19, 362-70 (2008).

21. Guilding C, Hughes A, Brown T, Namvar S, Piggins H. A riot of rhythms: neuronal and glial circadian oscillators in the mediobasal hypothalamus. Molecular Brain 2, 28 (2009).

22. Fick LJ, Fick GH, Belsham DD. Rhythmic clock and neuropeptide gene expression in hypothalamic mHypoE-44 neurons. Mol Cell Endocrinol 323, 298-306 (2010).

23. Akabayashi A, Levin N, Paez X, Alexander JT, Leibowitz SF. Hypothalamic neuropeptide $Y$ and its gene expression: relation to light/dark cycle and circulating corticosterone. Mol Cell Neurosci 5, 210-8 (1994).

24. Steiner RA, Kabigting E, Lent $\mathrm{K}$, Clifton DK. Diurnal rhythm in proopiomelanocortin mRNA in the arcuate nucleus of the male rat. J Neuroendocrinol 6, 603-8 (1994).

25. Xu B, Kalra PS, Farmerie WG, Kalra SP. Daily changes in hypothalamic gene expression of neuropeptide $\mathrm{Y}$, galanin, proopiomelanocortin, and adipocyte leptin gene expression and secretion: effects of food restriction. Endocrinology 140, 2868-75 (1999).

26. Wiater MF, Mukherjee S, Li AJ, Dinh TT, Rooney EM, et al. Circadian integration of sleep-wake and feeding requires NPY receptor-expressing neurons in the mediobasal hypothalamus. Am J Physiol Regul Integr Comp Physiol 301, R1569-83 (2011). 
27. Li AJ, Wiater MF, Oostrom MT, Smith BR, Wang $Q$, et al. Leptin-sensitive neurons in the arcuate nuclei contribute to endogenous feeding rhythms. Am J Physiol Regul Integr Comp Physiol 302, R1313-26 (2012).

28. Edelsbrunner ME, Painsipp E, Herzog $\mathrm{H}$, Holzer P. Evidence from knockout mice for distinct implications of neuropeptide-Y Y2 and Y4 receptors in the circadian control of locomotion, exploration, water and food intake. Neuropeptides 43, 491-7 (2009).

29. Tan K, Knight ZA, Friedman JM. Ablation of AgRP neurons impairs adaption to restricted feeding. Mol Metab 3, 694-704 (2014).

30. Mieda M, Williams SC, Sinton CM, Richardson JA, Sakurai T, Yanagisawa M. Orexin neurons function in an efferent pathway of a food-entrainable circadian oscillator in eliciting food-anticipatory activity and wakefulness. J Neurosci 24, 10493-501 (2004).

31. Richard CD, Tolle V, Low MJ. Meal pattern analysis in neural-specific proopiomelanocortin-deficient mice. Eur J Pharmacol 660, 131-8 (2011).

32. Sutton GM, Perez-Tilve D, Nogueiras R, Fang J, Kim JK, et al. The melanocortin-3 receptor is required for entrainment to meal intake. J Neurosci 28, 12946-55 (2008).

33. Welsh DK, Takahashi JS, Kay SA. Suprachiasmatic nucleus: cell autonomy and network properties. Annu Rev Physiol 72, 551-77 (2010).

34. Chou TC, Scammell TE, Gooley JJ, Gaus SE, Saper CB, Lu J. Critical role of dorsomedial hypothalamic nucleus in a wide range of behavioral circadian rhythms. J Neurosci 23, 10691-702 (2003).

35. Gooley JJ, Schomer A, Saper CB. The dorsomedial hypothalamic nucleus is critical for the expression of food-entrainable circadian rhythms. Nat Neurosci 9, 398-407 (2006).

36. Landry GJ, Simon MM, Webb IC, Mistlberger RE. Persistence of a behavioral food-anticipatory circadian rhythm following dorsomedial hypothalamic ablation in rats. Am J Physiol Regul Integr Comp Physiol 290, R1527-34 (2006).

37. Brochu-Gaudreau K, Rehfeldt C, Blouin R, Bordignon V, Murphy BD, Palin MF. Adiponectin action from head to toe. Endocrine 37, 11-32 (2010). 
38. Kubota N, Yano W, Kubota T, Yamauchi T, Itoh S, et al. Adiponectin stimulates AMP-activated protein kinase in the hypothalamus and increases food intake. Cell Metab 6, 55-68 (2007).

39. Liu J, Guo M, Zhang D, Cheng SY, Liu M, et al. Adiponectin is critical in determining susceptibility to depressive behaviors and has antidepressant-like activity. Proc Natl Acad Sci U S A 109, 12248-53 (2012).

40. Yamauchi T, Kadowaki T. Adiponectin receptor as a key player in healthy longevity and obesity-related diseases. Cell Metab 17, 185-96 (2013).

41. Brown SA, Fleury-Olela F, Nagoshi E, Hauser C, Juge C, et al. The period length of fibroblast circadian gene expression varies widely among human individuals. PLoS Biol 3, e338 (2005).

42. Balsalobre A, Brown SA, Marcacci L, Tronche F, Kellendonk C, et al. Resetting of circadian time in peripheral tissues by glucocorticoid signaling. Science 289, 2344-7 (2000).

43. Yoo SH, Yamazaki S, Lowrey PL, Shimomura K, Ko CH, et al. PERIOD2::LUCIFERASE real-time reporting of circadian dynamics reveals persistent circadian oscillations in mouse peripheral tissues. Proc Natl Acad Sci U S A 101, 5339-46 (2004).

44. Ma K, Cabrero A, Saha PK, Kojima H, Li L, et al. Increased beta -oxidation but no insulin resistance or glucose intolerance in mice lacking adiponectin. J Biol Chem 277, 34658-61 (2002).

45. Guo R, Zhang Y, Turdi S, Ren J. Adiponectin knockout accentuates high fat diet-induced obesity and cardiac dysfunction: role of autophagy. Biochim Biophys Acta 1832, 1136-48 (2013).

46. Bechtold DA, Loudon AS. Hypothalamic clocks and rhythms in feeding behaviour. Trends Neurosci 36, 74-82 (2013).

47. Kennaway DJ, Varcoe TJ, Voultsios A, Boden MJ. Global loss of bmal1 expression alters adipose tissue hormones, gene expression and glucose metabolism. PLOS ONE 8, e65255 (2013).

48. Mieda M, Sakurai T. Bmal1 in the nervous system is essential for normal adaptation of circadian locomotor activity and food intake to periodic feeding. J Neurosci 31, 15391-6 (2011).

49. Kadowaki T, Yamauchi T. Adiponectin and adiponectin receptors. Endocr Rev 26, 439-51 (2005). 
50. Schraw T, Wang ZV, Halberg N, Hawkins M, Scherer PE. Plasma adiponectin complexes have distinct biochemical characteristics. Endocrinology 149, 2270-82 (2008).

51. Iwabu M, Yamauchi T, Okada-Iwabu M, Sato K, Nakagawa T, et al. Adiponectin and AdipoR1 regulate PGC-1alpha and mitochondria by $\mathrm{Ca}(2+)$ and AMPK/SIRT1. Nature 464, 1313-9 (2010).

52. Liu C, Li S, Liu T, Borjigin J, Lin JD. Transcriptional coactivator PGC-1alpha integrates the mammalian clock and energy metabolism. Nature 447, 477-81 (2007).

53. Lee HK, McAlpine J, Chitta S, Bouzida D, Singh G, et al. Characterization of a small molecule compound VPR-66 as an inverse agonist for ROR proteins (P5167). J Immunol 190, 68.11 (2013).

54. Jager S, Handschin C, St-Pierre J, Spiegelman BM. AMP-activated protein kinase (AMPK) action in skeletal muscle via direct phosphorylation of PGC-1alpha. Proc Natl Acad Sci U S A 104, 12017-22 (2007).

55. Canto C, Gerhart-Hines Z, Feige JN, Lagouge M, Noriega L, et al. AMPK regulates energy expenditure by modulating NAD+ metabolism and SIRT1 activity. Nature 458, 1056-60 (2009).

56. Lamia KA, Sachdeva UM, DiTacchio L, Williams EC, Alvarez JG, et al. AMPK regulates the circadian clock by cryptochrome phosphorylation and degradation. Science 326, 437-40 (2009).

57. Hardie DG, Ross FA, Hawley SA. AMPK: a nutrient and energy sensor that maintains energy homeostasis. Nat Rev Mol Cell Biol 13, 251-62 (2012).

58. Fuller PM, Lu J, Saper CB. Differential rescue of light- and food-entrainable circadian rhythms. Science 320, 1074-7 (2008).

59. Mistlberger RE, Yamazaki S, Pendergast JS, Landry GJ, Takumi T, Nakamura W. Comment on "Differential rescue of light- and food-entrainable circadian rhythms". Science 322, 675; author reply (2008).

60. Liu M, Liu F. Transcriptional and post-translational regulation of adiponectin. Biochem J 425, 41-52 (2010).

61. Eckel-Mahan KL, Patel VR, de Mateo S, Orozco-Solis R, Ceglia NJ, et al. Reprogramming of the circadian clock by nutritional challenge. Cell 155, 1464-78 (2013). 
62. Preitner N, Damiola F, Lopez-Molina L, Zakany J, Duboule D, et al. The orphan nuclear receptor REV-ERBalpha controls circadian transcription within the positive limb of the mammalian circadian oscillator. Cell 110, 251-60 (2002).

63. Cho H, Zhao X, Hatori M, Yu RT, Barish GD, et al. Regulation of circadian behaviour and metabolism by REV-ERB-alpha and REV-ERB-beta. Nature 485, 123-7 (2012).

64. Solt LA, Wang Y, Banerjee S, Hughes T, Kojetin DJ, et al. Regulation of circadian behaviour and metabolism by synthetic REV-ERB agonists. Nature 485, 62-8 (2012).

65. Gervois P, Chopin-Delannoy S, Fadel A, Dubois G, Kosykh V, et al. Fibrates increase human REV-ERBalpha expression in liver via a novel peroxisome proliferator-activated receptor response element. Mol Endocrinol 13, 400-9 (1999).

66. Yamauchi T, Nio Y, Maki T, Kobayashi M, Takazawa T, et al. Targeted disruption of AdipoR1 and AdipoR2 causes abrogation of adiponectin binding and metabolic actions. Nat Med 13, 332-9 (2007).

67. Kim KY, Baek A, Hwang JE, Choi YA, Jeong J, et al. Adiponectin-activated AMPK stimulates dephosphorylation of AKT through protein phosphatase 2A activation. Cancer Res 69, 4018-26 (2009).

68. Liu Q, Gauthier MS, Sun L, Ruderman N, Lodish H. Activation of AMP-activated protein kinase signaling pathway by adiponectin and insulin in mouse adipocytes: requirement of acyl-CoA synthetases FATP1 and Acsl1 and association with an elevation in AMP/ATP ratio. FASEB J 24, 4229-39 (2010).

69. Ribeiro AC, Ceccarini G, Dupre C, Friedman JM, Pfaff DW, Mark AL. Contrasting effects of leptin on food anticipatory and total locomotor activity. PLOS ONE 6, e23364 (2011).

70. Mistlberger RE, Marchant EG. Enhanced food-anticipatory circadian rhythms in the genetically obese Zucker rat. Physiol Behav 66, 329-35 (1999).

71. LeSauter J, Hoque N, Weintraub M, Pfaff DW, Silver R. Stomach ghrelin-secreting cells as food-entrainable circadian clocks. Proc Natl Acad Sci U S A 106, 13582-7 (2009).

72. Blum ID, Patterson Z, Khazall R, Lamont EW, Sleeman MW, et al. Reduced anticipatory locomotor responses to scheduled meals in ghrelin receptor deficient mice. Neuroscience 164, 351-9 (2009). 
73. Szentirmai E, Kapas L, Sun Y, Smith RG, Krueger JM. Restricted feeding-induced sleep, activity, and body temperature changes in normal and preproghrelin-deficient mice. Am J Physiol Regul Integr Comp Physiol 298, R467-77 (2010).

74. Mieda M, Williams SC, Richardson JA, Tanaka K, Yanagisawa M. The dorsomedial hypothalamic nucleus as a putative food-entrainable circadian pacemaker. Proc Natl Acad Sci U S A 103, 12150-5 (2006).

75. Moriya T, Aida R, Kudo T, Akiyama M, Doi M, et al. The dorsomedial hypothalamic nucleus is not necessary for food-anticipatory circadian rhythms of behavior, temperature or clock gene expression in mice. Eur J Neurosci 29, 1447-60 (2009).

76. Turek FW, Joshu C, Kohsaka A, Lin E, Ivanova G, et al. Obesity and metabolic syndrome in circadian Clock mutant mice. Science 308, 1043-5 (2005).

77. Hashinaga T, Wada N, Otabe S, Yuan X, Kurita Y, et al. Modulation by adiponectin of circadian clock rhythmicity in model mice for metabolic syndrome. Endocr J 60, 483-92 (2013).

78. Paladino N, Mul Fedele ML, Duhart JM, Marpegan L, Golombek DA. Modulation of mammalian circadian rhythms by tumor necrosis factor-alpha. Chronobiol Int 31, 668-79 (2014).

79. Barnea M, Chapnik N, Genzer Y, Froy O. The circadian clock machinery controls adiponectin expression. Mol Cell Endocrinol 399, 284-7 (2015).

80. Allison KC, Lundgren JD, O'Reardon JP, Geliebter A, Gluck ME, et al. Proposed diagnostic criteria for night eating syndrome. Int J Eat Disord 43, 241-7 (2010).

81. Stunkard A, Berkowitz R, Wadden T, Tanrikut C, Reiss E, Young L. Binge eating disorder and the night-eating syndrome. Int J Obes Relat Metab Disord 20, 1-6 (1996).

82. Birketvedt GS, Florholmen J, Sundsfjord J, Osterud B, Dinges D, et al. Behavioral and neuroendocrine characteristics of the night-eating syndrome. JAMA 282, 657-63 (1999).

83. Goel N, Stunkard AJ, Rogers NL, Van Dongen HP, Allison KC, et al. Circadian rhythm profiles in women with night eating syndrome. J Biol Rhythms 24, 85-94 (2009).

84. Roenneberg T, Allebrandt KV, Merrow M, Vetter C. Social jetlag and obesity. Curr Biol 22, 939-43 (2012). 
85. Okada-Iwabu M, Yamauchi T, Iwabu M, Honma T, Hamagami K, et al. A small-molecule AdipoR agonist for type 2 diabetes and short life in obesity. Nature 503, 493-9 (2013).

86. Leliavski A, Shostak A, Husse J, Oster H. Impaired glucocorticoid production and response to stress in Arntl-deficient male mice. Endocrinology 155, 133-42 (2014).

87. Green MR, Sambrook J. 2012. Molecular Cloning: A Laboratory Manual (Fourth Edition). New York, USA: Cold Spring Harbor Laboratory Press

88. Storch KF, Weitz CJ. Daily rhythms of food-anticipatory behavioral activity do not require the known circadian clock. Proc Natl Acad Sci U S A 106, 6808-13 (2009).

89. Meng QJ, Logunova L, Maywood ES, Gallego M, Lebiecki J, et al. Setting clock speed in mammals: the CK1 epsilon tau mutation in mice accelerates circadian pacemakers by selectively destabilizing PERIOD proteins. Neuron 58, 78-88 (2008).

90. Zhang EE, Liu Y, Dentin R, Pongsawakul PY, Liu AC, et al. Cryptochrome mediates circadian regulation of cAMP signaling and hepatic gluconeogenesis. Nat Med 16, 1152-6 (2010).

91. lijima M, Yamaguchi S, van der Horst GT, Bonnefont X, Okamura H, Shibata S. Altered food-anticipatory activity rhythm in Cryptochrome-deficient mice. Neurosci Res 52, 166-73 (2005).

92. Feillet CA, Ripperger JA, Magnone MC, Dulloo A, Albrecht U, Challet E. Lack of food anticipation in Per2 mutant mice. Curr Biol 16, 2016-22 (2006).

93. Yang $\mathrm{S}$, Liu A, Weidenhammer A, Cooksey RC, McClain $\mathrm{D}$, et al. The role of mPer2 clock gene in glucocorticoid and feeding rhythms. Endocrinology 150, 2153-60 (2009).

94. Mendoza J, Albrecht U, Challet E. Behavioural food anticipation in clock genes deficient mice: confirming old phenotypes, describing new phenotypes. Genes Brain Behav 9, 467-77 (2010).

95. Dallmann R, Weaver DR. Altered body mass regulation in male mPeriod mutant mice on high-fat diet. Chronobiol Int 27, 1317-28 (2010).

96. Delezie J, Dumont S, Dardente H, Oudart H, Grechez-Cassiau A, et al. The nuclear receptor REV-ERBalpha is required for the daily balance of carbohydrate and lipid metabolism. FASEB $J \mathbf{2 6}$, 3321-35 (2012). 
97. Lau P, Fitzsimmons RL, Raichur S, Wang SC, Lechtken A, Muscat GE. The orphan nuclear receptor, RORalpha, regulates gene expression that controls lipid metabolism: staggerer (SG/SG) mice are resistant to diet-induced obesity. J Biol Chem 283, 18411-21 (2008). 


\section{Curriculum Vitae}

\section{Personal}

Name: Anthony Hiu King Tsang

Nationality: Hong Kong SAR \& British National (Oversea)

Language: English, Chinese, Cantonese

Mailing address: AG Chronophysiologie, Universitaet zu luebeck, Ratzeburger Allee 160, Haus 50, 23562, Luebeck, Germany

E-mail address: anthony.tsang@uksh.de (office) / ant.tsang@gmail.com (personal)

\section{Higher Education}

1. Doctoral candidate, Genes and Development Program, Goettingen Graduate School for Neurosciences and Molecular Biosciences, Georg-August University Goettingen, Germany and Max Planck Institute for Biophysical Chemistry, 2010 - Present

2. Master of Science (MSc)/ Doctor of Philosophy (PhD) student in Neuroscience Program (Successful fulfilment of coursework and examination), International Max Planck Research School, Georg-August University Goettingen, Germany, 2009 - 2010

3. Master of Philosophy (MPhil) in Biochemistry, The Hong Kong University of Science and Technology (HKUST), Hong Kong, 2006 - 2009

4. Bachelor of Science (BSc) in Biology with Honorous, The Chinese University of Hong Kong (CUHK), Hong Kong, 2003 - 2006 


\section{Publications (in reversed chronological order)}

\section{Research articles:}

1. Tsang A.H., Koch C.E., Leliavski A. and Oster H. (2014) Adiponectin regulates mediobasal hypothalamic circadian clock, feeding rhythms and adaptation to restricted feeding. (Manuscript under preparation)

2. Pagel R., Bär F., Schröder T., Langenstrassen P., Michaels M.A., Kalies K., König P., Grassl G.A., Meyer M., Tsang A.H., Büning J., Lehnert H., Fellermann K., Oster H. and Sina C. (2014) Circadian disruption impairs mucosal barrier function and promotes intestinal. (Manuscript under preparation)

3. Landgraf D.*, Tsang A.H., Leliavski A., Koch C.E., Barclay J.L., Drucker D.J. and Oster H. (2014) Oxyntomodulin regulates resetting of the liver circadian clock by food. (Manuscript under preparation)

4. Husse J., Leliavski A., Tsang A.H., Oster H., Eichele G. (2014) The light-dark cycle controls peripheral rhythmicity in mice with a genetically ablated suprachiasmatic nucleus clock. FASEB $J$ fj.14-256594. (Cover article of the issue)

5. Barclay J.L., Shostak. A., Leliavski A., Tsang A.H., Jöhren O., Müller-Fielitz H., Landgraf D., Naujokat N., van der Horst G.T. and Oster H. (2013) High fat diet-induced hyperinsulinemia and tissue-specific insulin resistance in Cry deficient mice. Am J Physiol Endocrinol Metab 304(10):E1053-63 (Highlighted with an editorial commentary by Oren Froy.)

6. Tsang A.H.*, Sánchez-Moreno C.* , Bode B., Rossner M.J., Garaulet M., and Oster H. (2012) Tissue-specific interaction of Per1/2 and Dec2 in the regulation of cellular circadian rhythms. J Biol Rhythms 27(6):478-89* Equal contributions

7. Zhou R., Tsang A.H., Lau E.S. and Ge W. (2012) Putitary adenylate cyclase-activating polypeptide (PACAP) and its receptors in the zebrafish ovary: evidence for potential dual roles of PACAP in controlling final oocyte maturation. Biol Reprod 85(3):615-25 (Cover article of the issue) 
8. Zhang P., Yu P.C., Tsang A.H., Chen Y., Fu A.K., Fu W.Y., Chung K.K., and Ip N.Y. (2010). S-nitrosylation of cyclin-dependent kinase 5 (CDK5) regulates its kinase activity and dendrite growth during neuronal development. J Neurosci 30(43):14366

9. Tsang A.H., Lee Y.I., Ko H.S., Savitt J. M., Pletnikova O. Troncoso J.C., Dawson V.L., Dawson T.M. and Chung K.K. (2009). S-nitrosylation of XIAP compromises neuronal survival in Parkinson's disease. Proc Natl Acad Sci U S A 106: 49005 (Recommended in F1000Prime by Richard Silverman; F1000Prime.com/1162958)

\section{Review articles and book chapters:}

1. Tsang A.H., Kolbe I., Seemann J., and Oster H. (2014) Interaction of circadian and stress systems in the regulation of adipose physiology. Horm Mol Biol Clin Investig 19(2):103-115

2. Tsang A.H., Barclay J.L., and Oster H. (2013) Interactions between endocrine and circadian systems. J Mol Endo 52(1):R1-R16.

3. Tsang A.H., Husse J., and Oster H. (2013) The role of sleep in the regulation of energy homeostasis and the development of the metabolic syndrome. In: Chronobiology and Obesity (Garaulet M. and Ordovás J.M., ed ), Springer pp.89-109

4. Barclay J.L., Tsang A.H., and Oster H. (2012) Interaction of central and peripheral clocks in physiological regulation. Prog Brain Res. 199:163-81

5. Tsang A.H. and Chung K.K. (2009). Oxidative stress and nitrosative stress in Parkinson's disease. Biochim Biophys Acta - Molecular Basis of Disease 1792(7):64350 


\section{Academic Awards}

1. Attendance to the $63^{\text {rd }}$ Lindau $63^{\text {rd }}$ Nobel Laureate Meeting 2013 - sponsored by Bayer-Lindau Fellowship, Bayer Science and Education Foundation, 2013

2. GGNB Excellence Stipend, Goettingen Graduate School for Neurosciences and Molecular Biosciences, Georg-August University Goettingen, 2011 - Present

3. Stipend of the Excellence Foundation for the Promotion of the Max Planck Society, International Max Planck Research School, 2009 - 2010

4. University Grant Council (UGC) Research Travel Grant, The Hong Kong University of Science and Technology, 2008

5. Research Postgraduate Studentship, The Hong Kong University of Science and Technology, $2006-2009$

6. Certificate of Academic Merit, Shaw College, The Chinese University of Hong Kong, 2005 2006

7. Dean's Honours List (annual), The Chinese University of Hong Kong, 2005 - 2006

8. Dean's Honours List (annual), The Chinese University of Hong Kong, 2004 - 2005

9. The Department/ Program Scholarship, Shaw College, The Chinese University of Hong Kong $2004-2005$ 


\section{Research Experience}

1. Doctoral thesis research student under supervision of Prof. Henrik Oster, Circadian Rhythm Group, Max Planck Institute for Biophysical Chemistry (and later Chronophysiology Research Group, Universität zu Lübeck) 12/2010 — present (Thesis title: Metabolic resetting of circadian clocks in central nervous system)

2. Visiting Scholar with Prof. Kenny Chung, Department of Biochemistry, the Hong Kong University of Science and Technology 02/2009 - 07/2009

3. Master thesis research student under supervision of Prof. Kenny Chung, Department of Biochemistry, the Hong Kong University of Science and Technology 06/2006 — 01/2009 (Thesis title: S-nitrosylation of XIAP: Implication for the pathogenesis of Parkinson's disease)

4. Bachelor thesis research student under supervision of Prof. Wei Ge, Department of Biology, the Chinese University of Hong Kong 06/2005 — 06/2006 (Thesis title: Dual role of PACAP in zebrafish oocyte maturation)

5. Laboratory Helper in Prof. Siu Wai CHIU's laboratory, Department of Biology, the Chinese University of Hong Kong 04/2004 — 10/2005

\section{Scientific Activities}

1. Participation and poster presentation, The $63^{\text {rd }}$ Lindau Nobel Laureate Meeting 2013 (Chemistry), Lindau, Germany. 30 Jun - 21 Jul 2013 
2. Participation and poster presentation, the Chronobiology Summer School 2012, Berlin, Germany. 16 - 21 Sep 2012

3. Poster presentation, the Society of Neuroscience (SFN) annual meeting 2008, Washington DC, USA. 15 - 19 Nov 2008

4. Poster presentation, the Gordon Research Conference "Molecular and Cellular Neurobiology" 2008, HKUST, Hong Kong SAR, China, 08 - 13 Jun 2008

\section{Professional skills with hand-on experience}

Cell line and primary cell culture;

Electrophysiology (patch clamp technique);

Ex vivo brain-slice culture;

Fluorescence assisted cell sorting (FACS) analysis;

Fluorescent/Luminescence microscopy;

Lenti-/ Adeno-associated viral vector mediated gene transfer;

Mouse behavioral experiment;

Mouse handling and genetics;

Mouse stereotaxic surgery;

Molecular cloning;

Recombinant protein purification and Fast protein liquid chromatography (FPLC);

General techniques in biochemistry and molecular biology 


\section{Acknowledgement}

I would like to thank my supervisor Prof. Dr. Henrik Oster for bringing me this exciting project and for his patient guidance as well as his tolerance for my ignorance. He has been giving me enormous support throughout my study.

I am grateful to have Prof. Dr. Thomas Pieler and Prof. Dr. Moritz Rossner as my thesis supervisory committee members from whom I have gained uncountable invaluable advices to my project from the angles of their own expertise.

I am also grateful for the encouragements and thoughtful suggestions from all my labmates. Particularly, I feel in debt with Dr. Christiane Koch who has contributes excellent collaborative efforts in the stereotaxic i.c.v. and tail-vein injection experiments in the current study.

I would like to thank for the funding agencies of this thesis project as well. I am a recipient of the GGNB Excellence Stipend of the University of Göttingen. Thus this work was partly supported by the Göttingen Graduate School for Neurosciences, Biophysics and Molecular Biosciences (DFG Grants GSC 226/1, GSC 226/2) and also by Volkswagen Foundation to Prof. Henrik Oster. 


\title{
Selected publications related to the current study
}

\begin{tabular}{l|l|l|l|l|} 
Review & A H TSANG and others & Endocrine circadian rhythms & 52:1 & R1-R16 \\
\cline { 2 - 3 } & &
\end{tabular}

\section{Interactions between endocrine and circadian systems}

\author{
Anthony H Tsang ${ }^{1,2}$, Johanna L Barclay ${ }^{1,3}$ and Henrik Oster ${ }^{1,2}$ \\ 'Circadian khythms Group, Max Planck Institute for Biophysical Chemistry, Göttingen, Germany \\ ${ }^{2}$ Chronophysiology Group, Medical Department I, University of Lübeck, Ratzeburger Allee 160, \\ 23538 Lübeck, Germany \\ ${ }^{3}$ School of Medicine, University of Queensland, Brisbane, Queensland, Australia
}

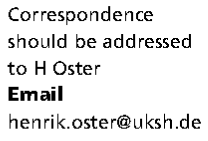

Correspondence

should be addressed

to $\mathrm{H}$ Oster

Email

henrik.oster@uksh.de

\section{Abstract} physiological and metabolic homeostasis.

\section{Introduction}

We live in an environment shaped by various geophysical rhythms. Arguably, one of the most prominent of these rhythms is the succession of day and night. The profound environmental changes brought about by the rotation of the Earth around its axis have promoted the development of endogenous timekeepers that enable an organism to reliably predict the time of day and adjust behavior and physiology accordingly. Not surprisingly, large aspects of our endocrine system are tightly connected to the circadian (from Latin circa diem - about a day) clock. With recent advances in molecular life sciences and medicine, we now realize that this interaction is not only unilateral but also includes endocrine feedback on circadian clock function. This review recapitulates some of the research leading to the picture we have today of the circadian clock system in mammals and provides an overview about the most prominent connection points between circadian and endocrine regulation.
\end{abstract}

In most species, endogenous circadian clocks regulate 24-h rhythms of behavior and physiology. Clock disruption has been associated with decreased cognitive performance and increased propensity to develop obesity, diabetes, and cancer. Many hormonal factors show robust diurnal secretion rhythms, some of which are involved in mediating clock output from the brain to peripheral tissues. In this review, we describe the mechanisms of clock-hormone interaction in mammals, the contribution of different tissue oscillators to hormonal regulation, and how changes in circadian timing impinge on endocrine signalling and downstream processes. We further summarize recent findings suggesting that hormonal signals may feed back on circadian regulation and how this crosstalk interferes with

$$
\begin{aligned}
& \text { http://Ime.endocrinology-journals.org } \\
& \text { DOI: } 10.1530 / \mathrm{MM}-13-0118
\end{aligned}
$$

DOI: $10.1530 / \mathrm{M} \mathrm{M}-13-0118$
(9) 2014 Society for Endocrinology Pociety for Endocrinology
Printed in Great Britain

\section{Key Words}

- circadian clocks

- cortisol

- endocrine rhythm

- melatonin

- adipokines
Journal of Molecular
Endocrinology Endocrinology
(2014) 52, R1-R16

\section{The master circadian pacemaker}

In the 1970 s, we witnessed a significant breakthrough in the field of chronobiology - the identification of the anatomical entity underlining the mammalian circadian rhythm. It was discovered that information about the external light-dark cycle was passed via the retinohypothalamic tract (RHT) to not only sensory input integrating centers in the thalamus, but also to the hypothalamic suprachiasmatic nucleus (SCN), hinting at the existence of a novel photic input processing hub in the brain (Sousa-Pinto \& Castro-Correia 1970, Hendrickson et al. 1972, Moore \& Lenn 1972). The SCN is a bilaterally paired structure with high cell body density located adjacent to the third ventricle and directly atop the optic chiasm. It comprises about 50000 neurons in humans and about 20000 neurons in rodents. A series of electrical lesion studies provided unequivocal evidence for the

Published by Eioscientifica Ltd. 
critical role of SCN in the generation of mammalian circadian rhythms. Animals with ablated SCN become behaviorally and physiologically arrhythmic (Moore \& Eichler 1972, Stephan \& Zucker 1972). Critically, transplanting isolated SCN tissue into SCN-lesioned animals restores circadian rhythmicity (Ralph et al. 1990), and the restored behavioral rhythm of recipients is determined by the donor's intrinsic period, indicating that the SCN is indeed the master pacemaker generating circadian timing information in aninals (Ralph te al. 1990). Brain slice explants of the SCN, but not of other tested brain areas including the cerebral cortex and arcuate nucleus, display robust circadian oscillations in firing rate in vitro, suggesting that the rhythmicity of the SCN is autonomous and self-sustaining (Green \& Gillette 1982, Groos \& Hendriks 1982, Shibata et al. 1982).

\section{The molecular clockwork}

The Period (or Per) gene was the first discovered clock gene (Konopka \& Benzer 1971), which is conserved from fruit flies to humans. Mutations of Per in flies alters the circadian patterns of pupae eclosion and locomotor activity (Konopka \& Benzer 1971). Since then, many more clock genes have heen identified in different organisms (Zhang \& Kay 2010). In the past decades, our knowledge of the molecular clockwork has been significantly expanded. The current model suggests that the central mechanism of the mammalian molecular clock is composed of a set of clock genes intertwined with a delayed interlocking transcriptional-translational feedback loop (TTL), coupled to several auxiliary mechanisms reinforcing robustness and stability (Zhang \& Kay 2010). The positive limb of this TTL comprises two basic helixloop-helix transcription factors, circadian locomotor output cycles kaput (CLOCK), and brain and muscle aryl hydrocarbon receptor nuclear translocator such as BMAL1 or ARNTL. Both form heterodimers via their PER-ARNTSIM (PAS) domains and activate E-box-element-containing genes by recruiting transcriptional co-activators, chromatinmodifying proteins, and RNA polymerase II. In certain tissues such as the forebrain or the vasculature, CLOCK is functionally replaced by its homolog neuronal PAS domain protein 2 (NPAS2; McNamara et al. 2001, Reick et al. 2001). Period (Per1-3) and Cryptochome (Cry1/2) constitute the negative limb of the TTL. CLOCK-BMAL1 complexes activate the transcription of Per and Cry genes during the subjective day. PERs and CRYs translocate into the nucleus and form inhibitory complexes. With progress of the circadian cycle, PER/CRY complexes accumulate

\begin{tabular}{|lr}
\hline http://Ime.endocrinology-journals.org & 92014 Society for Endocrinology \\
DOI: $10.1530 / \mathrm{ME}-13-0118$ & Printed in Great Britain
\end{tabular}

and so does their inhibitory effect on CLOCK-BMAL1 activity, shutting down Per and Cry transcription during the night (Lee et al. 2001). The progressive degradation of PER/CRY complexes throughout the night toward the morning releases the inhibition on CLOCK-BMAL1 transcriptional activity and thereby, completes the negative feedback loop of the circadian clock (Fig. 1).

Additional auxiliary TTLs enhance the stability of the core clock TTL and translate time-of-day information into physiological signals via transcriptional control of clock target genes (Zhang \& Kay 2010). Such loops include the nuclear receptors REV-ERB $\alpha$ and REV-ERB $\beta$ (NR1D1 and NR1D2) and $R O R \alpha$ (NR1F1) which regulate Bmal1 expression via a retinoid orphan receptor responsive elements (Preitner et al. 2002, Ueda et al. 2002, Sato et al. 2004, Akashi \& Takumi 2005, Liu et al. 2008), as well as the PAR basic leucine zipper proteins D-box albumin-binding protein and $\mathrm{E} 4$ promoter-binding protein (E4BP; NFIL3) (Cowell 2002, Ripperger \& Schibler 2006) which feed-back on the expression of Per genes via D-box promoter elements (Ripperger et al. 2000).

\section{Extra-SCN oscillators}

The functional molecular clockwork exists not only in SCN neurons, but (almost) every single cell in the brain and periphery is capable of oscillating in a circadian manner. Molecular clock rhythms have been shown even in cultured cells, such as immortalized fibroblast cells which display robust oscillations of clock gene expression

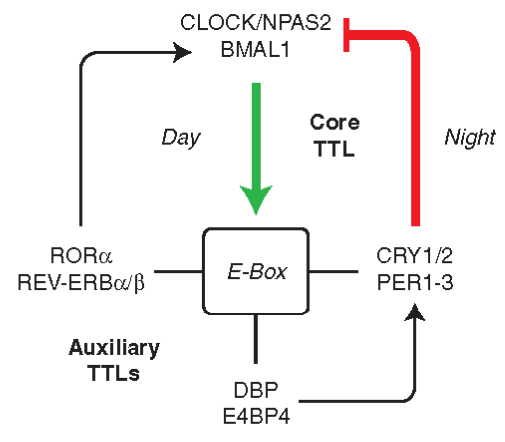

Figure 1

The molecular mammalian circadian clockwork. The transcription factors Clock/Npas2 and Bmal1 activate E-box-controlled genes including PER1-3 and $C R Y 1 / 2$ during the day. PER and CRY proteins inhibit CLOCK/BMAL1 activity during the night. Auxiliary loops stabilize this 24-h rhythm of transcriptional activation by modulating gene expression of Bmal1 and Per. For details see text

Published by Bioscientifica Ltd. 


\section{Review}

after a brief stimulation with high concentrations of serum (Balsalobre et al. 1998). Using single cell imaging techniques, Nagoshi et al. (2004) showed that each fibroblast cell possesses a sustained circadian clock, although at the population level the rhythm dampens quickly as a consequence of a gradual desynchronization between individual cells with different endogenous periods. Application of synchronizing agents such as serum, forskolin, glucocorticoids (GCs), or phorbol esters re-synchronizes individual cells, yielding a transiently phase-coherent population (Nagoshi et al. 2004). These data suggest that the cellular clocks in extra-SCN tissues are actually self-sustained and autonomous in nature, but fail to maintain coherence at the population level, in contrast to the SCN (see below). Similarly, tissue explants from a wide array of peripheral organs including heart, lung, kidney, liver, spleen, pancreas, stomach, cornea, thyroid gland, and adrenal gland show clock gene expression rhythms, but the overall rhythm dampens quickly due to the gradual loss of coherence between individual cells (Yamazaki et al. 2000, Yoo et al. 2004). Similar results have been obtained from tissue explants from various brain regions (Abe et al. 2002, Guilding \& Piggins 2007).

\section{SCN communication}

In order to achieve a biologically relevant circadian rhythm, it is of utmost importance that individual cells of a specific tissue are synchronized to the external environment. The circadian oscillation of an SCN neuron is coupled to its neighbouring cells in an action-potentialdependent manner (Welsh et al. 1995). This intercellular coupling property bestows the superior robustness and resilience of the SCN circadian rhythm. For example, the SCN explant cultures exhibit robust and persistent circadian oscillations in electrophysiological activity and clock gene expression for an extended period of time, while rhythms in slices from most other brain regions and peripheral tissues dampen after a couple of days (Guilding \& Piggins 2007, Guilding et al. 2009). SCN explant rhythms are also more resistant to clock gene mutations (Liu et al. 2007) and temperature fluctuations (Abraham et al. 2010, Buhr et al. 2010). One major function of the $\mathrm{SCN}$ is to synchronize internal biological processes to external time cues. The SCN receives photic information from both classical photoreceptors - cone and rod cells - as well as melanopsin-containing retinal ganglion cells via the RHT (Hankins et al. 2008). In turn, the SCN innovates other regions of the brain, in particular the hypothalamus.

\begin{tabular}{|lr}
\hline http://Ime.endocrinology-journals.org & 92014 Society for Endocrinology \\
DOI: $10.1530 / \mathrm{ME}-13-0118$ & Printed in Great Britain
\end{tabular}

The paraventricular hypothalamic nucleus (PVN) is one of the major loci relaying circadian information from the SCN to the rest of the body (Saeb-Parsy et al. 2000). The PVN is an important integrating center for energy homeostasis, projecting parvocellular neurons to the median eminence to control the release of hormones such as adrenocorticotrophin (ACTH) and thyroid-stimulating hormone in the anterior pituitary. The PVN also innervates the sympathetic limb of the autonomous nervous systen, thereby allowing the SCN to regulate the sympathetic tone of the body over the course of the day (Buijs et al. 2003). Further projections of the SCN to the dorsomedial hypothalamic nucleus (Luiten et al. 1987), the nucleus accumbens (Phillipson \& Griffiths 1985) and the paraventricular thalamic nucleus (Watts \& Swanson 1987, Watts et al. 1987) have been described. These connections enable the SCN to exert influence on a plethora of physiological processes such as the reward system, feeding-fasting cycles, cognitive function, locomotor activity, and body temperature (Dibner et al. 2010). In addition to direct neural connections, the SCN secretes diffusible factors, which can function as timing cues. Membrane-encapsulated foetal SCN tissue grafts, which allow only low-molecular-weight particles to diffuse, can restore the rhythmicity of locomotor activity in SC.Nlesioned hamsters in the absence of axonal outgrowth (Silver et al. 1996). Transforming growth factor- $\alpha$ (Kramer et al. 2001, Li et al. 2002), prokineticin 2 (PK2; Cheng et al. 2002), and cardiotropin-like cytokine (Kraves \& Weitz 2006) have been implicated as SCN-secreted peptides capable of regulating behavioral rhythmicity. Given the physical proximity of the SCN to the third ventricle, these diffusible factors may help propagate the time-of-day information to more remote brain regions via the cerebrospinal ventricular system.

\section{Endocrine rhythms: clock vs behavioral regulation}

It has been long been appreciated that the circulating levels of a number of hormones vary over the 24 -h cycle (Andrews \& Folk 1964). Such a diurnal rhythm of a hormone or metabolite can either be a manifestation of circadian clock control or a direct or indirect response to an environmental rhythm such as the light-dark cycle. Two methodologies have been developed to track down the relative contribution of the endogenous circadian clock to diurnal hormonal rhythms in humans, namely constant routine (CR) and forced desynchrony (FD) protocols. The CR protocol aims to minimize the effects of external time cues

Published by Bioscientifica Ltd. 
and behavioral variables by equally distributing such variables across the circadian cycle. Depending on individual experimental goals, it routinely demands constant wakefulness, limited physical activity, equally distributed isocaloric snacks or constant glucose infusion and constant dim light condition (Mills et al. 1978). The FD protocol employs a strategy which schedules a behavioral cycle beyond the entrainable range of the circadian clock (i.e. significantly longer or shorter than $24 \mathrm{~h}$ ) in a constant dinn light environnent, resulting in the free rumning of the endogenous circadian clock. This leads to an even distribution of certain behavioral variables in question across different phase of the circadian cycle (Kleitman 1970).

The clarification of the relative contribution of endogenous and exogenous input to the diurnal rhythm of a physiological system is of particular relevance for understanding the influence of our modern 24/7 lifestyle on the well-being of individuals. Owing to social constraints many rhythmic behaviors such as sleep/wake and food intake/fasting cycles often no longer align with their endogenous pattern controlled by the circadian clock (Scheer et al. 2009, Beccuti \& Pannain 2011). Shift workers are an obvious example. Several epidemiological studies indicate that shift workers are predisposed for metaholic disorders and even cancer (Ohayon ef al. 2000 , Akerstedt 2003). Thus, better knowledge for the mechanistic link between circadian misalignment and hormonal deregulation may help with the development of novel medical regimes to prevent or intervene in the metabolic consequences of shift work.

GCs and melatonin represent two well-studied hormonal systems that are subject to direct and dominant regulation by the circadian clock. The circulating levels of both display robust diurnal patterns (Migeon et al. 1956, Ralph et al. 1971). Using the CR and FD experimental protocols, the secretion rhythms of cortisol (el-Hajj Fuleihan et al. 1997, Wehr et al. 2001, Aeschbach et al. 2003, Scheer et al. 2010) and melatonin (Dijk et al. 1999, Wehr et al. 2001, Cain et al. 2010, Gooley et al. 2011) have been shown to be under direct regulation by the circadian clock. Not surprisingly, both hormones also act as major hormonal output pathways that propagate the time signal from the SCN to various other tissues. In the following paragraphs, we will discuss the interaction between the central clock and these two endocrine systems.

\section{SCN-adrenal interaction}

The adrenal gland is an endocrine organ composed of two anatomically distinct structures - the cortex and medulla.

\begin{tabular}{|lr}
\hline http://Ime.endocrinology-journals.org & 92014 Society for Endocrinology \\
DOI: $10.1530 / \mathrm{ME}-13-0118$ & Printed in Great Britain
\end{tabular}

The cortical part produces multiple corticosteroid hormones, while the medulla produces epinephrine and norepinephrine. The adrenal cortex is further organized into three functionally distinct subregions: the outermost zona glomerulosa producing mineralocorticoids, the middle zona fasciculata producing GCs (mainly cortisol in humans, corticosterone in rodents) and the innermost androgen-producing zona reticulata. A diurnal rhythm of the excretion of urinary ketosteroids was reported in the Inid 20th century (Pincus et al. 1954). In the 1970s, along with the identification of the SCN as the master circadian pacemaker, the circadian secretion of corticosteroids was established as a robust hormonal output of the SCN clock (Moore \& Eichler 1972, Liu et al. 2008). Only during the last decade, however, has the anatomical and molecular basis underlying the circadian production of corticosteroids been unveiled. Cholesterol is the precursor for the biosynthesis of steroid hormones. LDL - bound cholesterol - is imported into adrenocortical cells via LDL receptors. Cholesterol is then transported into mitochondria via steroidogenic acute regulatory protein (STAR). This import constitutes the rate-limiting step of steroidogenesis (Miller \& Bose 2011). Inside the mitochondria, the side chain of cholesterol is first removed by cytochrome p450sce to herome pregnenolone, which is then subjected to a series of enzyme-regulated reactions to become GC (Miller \& Bose 2011). GC secretion is highly stress responsive. Together with epinephrine, GCs boost energy production and prepare the body for foraging and fight-or-flight situations. GCs exert effects on a wide array of physiological systems. In times of high energy demand, GCs help maintain blood glucose levels by promoting gluconeogenesis in liver and lipolysis in adipose tissues (Kwon \& Hermayer 2013). GCs also play an important role in modulating immune (Silverman \& Sternberg 2012) and cognitive functions (Sandi 2011). The majority of the effects of GCs are mediated by its ubiquitously expressed cognate nuclear receptors, glucocorticoid receptors (GRs) (Silverman \& Sternberg 2012). Interestingly, despite the widespread expression pattern of GR within the brain, the SCN is devoid of GR (Okamura 2007).

The secretion of GC is the end product of hypothalamic-pituitary-adrenal (HPA) axis activation. Pituitaryreleased ACTH activates adrenocortical steroidogenesis through the melanocortin 2 receptor (MC2R), via a CAMP-PKA-dependent pathway which transcriptionally stimulates steroidogenic genes such as STAR and CYP11A1 (Miller \& Bose 2011).

Blood levels of GCs display a robust circadian rhythm, overlaid by less regular ultradian pulses with a period of

Published by Bioscientifica Ltd. 
90-120 min. The circadian rise of GCs is phase-locked to the time of awakening, peaking at few hours before the onset of the active phase, i.e. the early morning for diurnal animals such as humans and the evening for nocturnal animals such as mice (Moore \& Eichler 1972, GomezAbellan et al. 2012). This GC rise promotes arousal and boosts performance during the early active phase. Importantly, GC rhythms persist under constant environmental conditions, suggesting that they are driven by the endogenous circadian clock. Surgical ablation of the SCN completely abolishes the circadian rhythm of GC in blood, indicating that the SCN is the origin of GC rhythmicity (Moore \& Eichler 1972, Stephan \& Zucker 1972). HPA axis activity upstream of the adrenal is also rhythmic (Watts et al. 2004, Henley et al. 2009), which led to the hypothesis that circadian regulation of GC release may be an indirect response to $\mathrm{SCN}$-induced corticotrophin-releasing hormone (CRH) expression. However, this view has been challenged by several observations. First, the timing of $C R H$ expression in the hypothalamus of pro-opiomelanocortin (POMC; precursor peptide of ACTH) in the anterior pituitary and the plasma GC surge are not organized in the expected sequential manner (Watts et al. 2004, Girotti et al. 2009). Also, implantation of ACTH pellets can restore the rhythmicity of GC in hypophysectomized rats, while denervation of the adrenal gland abolishes the daily GC rhythm, suggesting that ACTH rhythmicity per se is dispensable for the blood GC rhythm (Ottenweller et al. 1978, Ottenweller \& Meier 1982). Conversely, stimulation of adrenal sympathetic nerves results in potentiated GC responses which can be abolished by hypophysectomy (Edwards \& Jones 1993), suggesting a permissive function of pituitary-derived ACTH and a more direct role of sympathetic innervation in the regulation of the circadian $\mathrm{GC}$ rhythm. Indeed, it has been shown in viral tracing experiments that the adrenal is connected to the SCN via the spinal cord and the PVN (Buijs et al. 1999). In a more recent study, it has been shown that light signals are transmitted to the adrenal cortex via the SCN, inducing an up-regulation of PER1 expression and secretion of GC independent of ACTH (Ishida et al. 2005).

Well before the discovery of clock genes or peripheral clocks, it was shown that adrenal glands when isolated and cultured in vitro display a robust circadian rhythm of metabolism and steroid secretion (Andrews \& Folk 1964). In line with this, we now know that about $5 \%$ of the whole genome - including all canonical clock genes - show rhythmic expression in the mouse adrenal gland (Oster et al. 2006a). By transplanting adrenal glands from arrhythmic PER2/CRY1 double mutant mice to WT

\begin{tabular}{|lr} 
http://Ime.endocrinology-journals.org & 92014 Society for Endocrinology \\
DOl: 10.1530/JME-13-0118 & Printed in Great Britain
\end{tabular}

adrenalectomized mice, and vice versa, we have provided evidence that a local adrenocortical clock imposes a circadian gating mechanism altering ACTH sensitivity during the course of the day (Oster et al. 2006b). This observation was further supported in a study that used a knock down of BMAL1 in the adrenal cortex (Son et al. 2008). Taken together, this illustrates that while the SCN is indispensable for the circadian rhythm of GC secretion, the adrenal clock provides an additional level of control to modulate the proficiency of GC production across the circadian cycle and further clocks along the HPA axis may be involved (Fig. 2).

\section{SCN-pineal interaction}

Unlike mice and humans, many non-mammalian vertebrates can perceive photic information by extraretinal photoreceptors (Menaker et al. 1997, Foster \& Soni 1998), e.g. in the pineal. The pineal gland is an ancient organ that exists in most vertebrates (Menaker et al. 1997). In mammals, it is buried deep beneath the skull and lies within the furrow of the two hemispheres. In consequence, its photoreceptive function is lost. However, in most cases its physiology is still strongly influenced by light. A major function of the pineal is its secretion of the hormone melatonin derived from the amino acid tryptophan (Barrett \& Bolborea 2012). In mammals, melatonin exerts its effects via binding to its two widely expressed cognate receptors - MT1 and MT2. The melatonin receptors belong to the $\mathrm{G} \alpha_{\mathrm{i}} / \mathrm{q}_{\mathrm{i}}$-protein-coupled receptor superfamily (Barrett \& Bolborea 2012). Owing to the widespread expression of melatonin receptors, melatonin has been reported to modulate several physiological systems such as immune function (Srinivasan et al. 2011), metabolism (Nduhirabandi et al. 2012), and higher brain functions (Srinivasan et al. 2012). In birds and reptiles, the pineal-melatonin system is an essential part of the circadian clockwork (Gaston \& Menaker 1968, Tosini \& Menaker 1998). In contrast, no overt circadian disruption is observed in pinealectomized mammals (Quay 1970, 1972), but melatonin may play an important regulatory role in distributing the time signal of the SCN (see below).

The daily pattern of melatonin secretion profile has a robust profile - being low during the day; rising and peaking during the night. In contrast to the GC rhythm which is anti-phasic in nocturnal and diurnal animals, high melatonin is always confined to the dark phase. SCN lesions abolish melatonin rhythms (Klein \& Moore 1979, Reppert et al. 1981). The SCN connects to the

Published by Bioscientifica Ltd. 


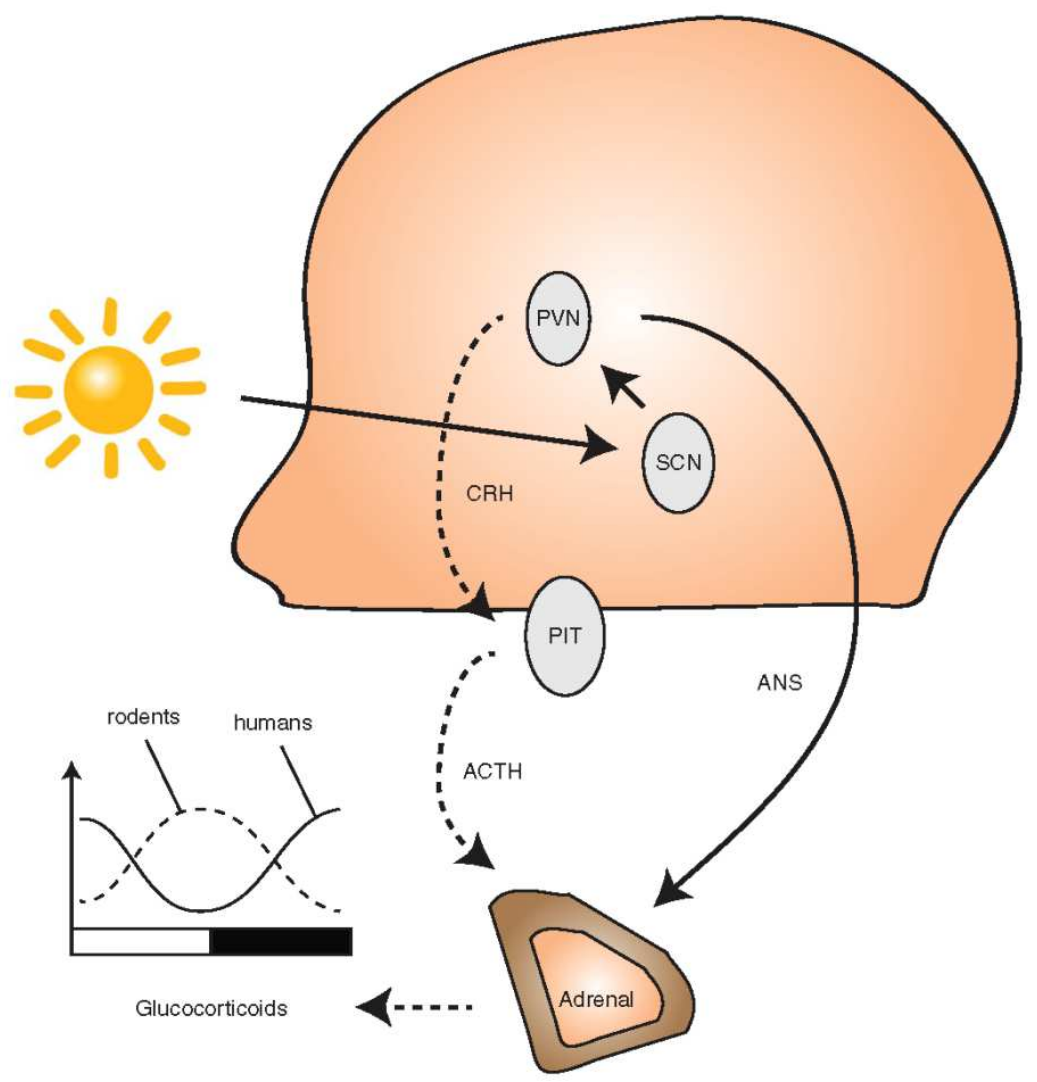

Figure 2

Interaction of central and peripheral clocks in the regulation of $\mathrm{GC}$ secretion. The $\mathrm{SCN}$ innervates the PVN from where rhythmic CRH release triggers secretion of ACTH from the pituitary (PIT). At the same time autonomic innervation (ANS) of the adrenal resets adrenocortical clocks regulating sensitivity of the steroidogenic machinery to ACTH.

Synchrony between HPA axis activity and adrenal ACTH gating results in high amplitude and robust circadian GC rhythms. GC rhythms are phaseshifted between nocturnal and diurnal species indicating differential interpretation of $\mathrm{SCN}$ signals at downstream targets.

pineal gland via a multi-synaptic autonomic pathway which sequentially involves the PVN and then the preganglionic neurons of the intermediolateral cell column of the spinal cord and finally the noradrenergic sympathetic neurons of the superior cervical ganglion (Drijfhout et al. 1996, Moore 1996, Larsen et al. 1998, Teclemariam-Mesbah et al. 1999; Fig. 3). The SCN releases GABA to inhibit the sympathetic input to the pineal gland during the daytime while this inhibition is released during the night (Kalsbeek et al. 2000). In addition, the SCN sends a constant glutamatergic stimulatory input to the pineal gland which is

http://Ime.endocrinology-journals.org DOI: 10.1530/JME-13-0118 Q 2014 Society for Endocrinology
Printed in Great Britain overwhelmed by the inhibitory mechanism during the night (Perreau-Lenz et al. 2004).

The role of clock genes in regulating pineal gland rhythmicity has received little attention, mainly due to the fact that many of the mouse genetic models used to study the function of the molecular clock are maintained on genetic backgrounds carrying mutations in two key enzymes of melatonin synthesis, arylalkylamine $\mathrm{N}$-acetyltransferase (AANAT) and hydroxyindole-O-methyltransferase (HIOMT), resulting in melatonin deficiency (Goto et al. 1989, Roseboom et al. 1998, Vivien-Roels et al. 1998). Clock- $\Delta 19$ mutants (Vitaterna et al. 1994) were

Published by Bioscientifica Ltd. 


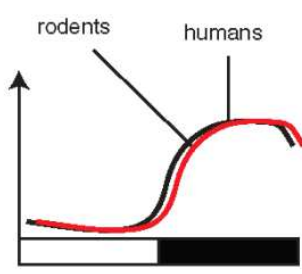

Melatonin
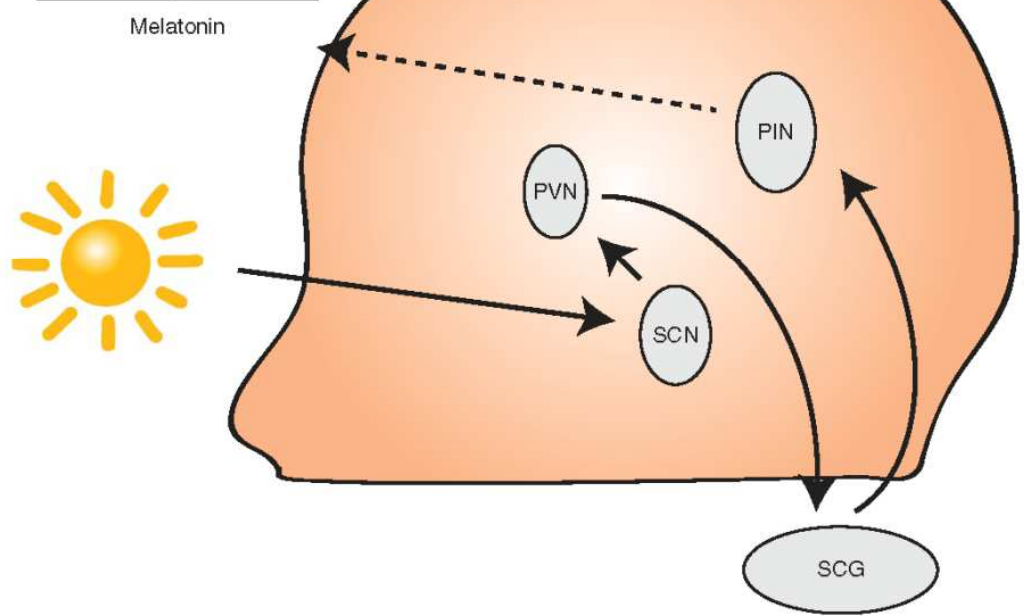

Figure 3

Melatonin release from the pineal is driven by the $\mathrm{SCN}$ pacemaker. The $\mathrm{SCN}$ innervates the PVN from where autonomous fibres descend into the spinal cord and out via the superior cervical ganglia (SCG) to reach the pineal gland (PIN). Clock genes are expressed in the pineal, but a functional

back-crossed into a melatonin-proficient strain, showing that the Clock- $\Delta 19$ mutation leads to phase delays and dampening of the melatonin rhythm in constant darkness conditions while GC rhythms were completely abolished (Kennaway et al. 2003, 2006). More recently, it has been demonstrated that the melatonin biosynthesis pathway can genetically suppress the circadian perturbations of Clock- $\Delta 19$ mutation (Shimomura et al. 2010), suggesting a role of melatonin in contributing to the robustness of the SCN clock (see below). PER1 deficiency has been shown to enhance Aanat transcription, enzymatic activity and hence melatonin secretion (Chen \& Baler 2000, Christ et al. 2010). In $C R Y 1 / 2$ double-deficient mice on a melatonin-proficient genetic background not only is the melatonin rhythm blunted under light-dark conditions, but also photic suppression of melatonin is abolished (Yamanaka et al. 2010). Together, these data suggest that clock genes impinge on pineal melatonin

http:///me.endocrinology-journals.org
DOI: $10.1530 / \mathrm{MME}-13-0118$

contribution of a potential pineal clock to melatonin production has not been demonstrated. Unlike GCs, melatonin secretion is always confined to the dark phase, regardless of the activity profile of the animal. rhythmicity. However, owing to the lack of suitable genetic models to study the tissue-specific function of clock genes in melatonin-proficient strains, the physiological role of the molecular clock in the pineal itself remains largely unclear.

\section{Hormonal feedback to the circadian clock}

The stabilizing role of melatonin in SCN regulation mentioned above suggests that hormonal rhythms - we have discussed circadian regulation of GC and melatonin secretion - are not merely an output of the central clock. They can also feedback to the various levels of the circadian system and thereby intervene the circadian rhythm of physiology and behavior of animals (Fig. 4). In the following section, we will use these and some other hormones as examples to illustrate the crosstalk within the clock-hormones circuitry in mammals. 


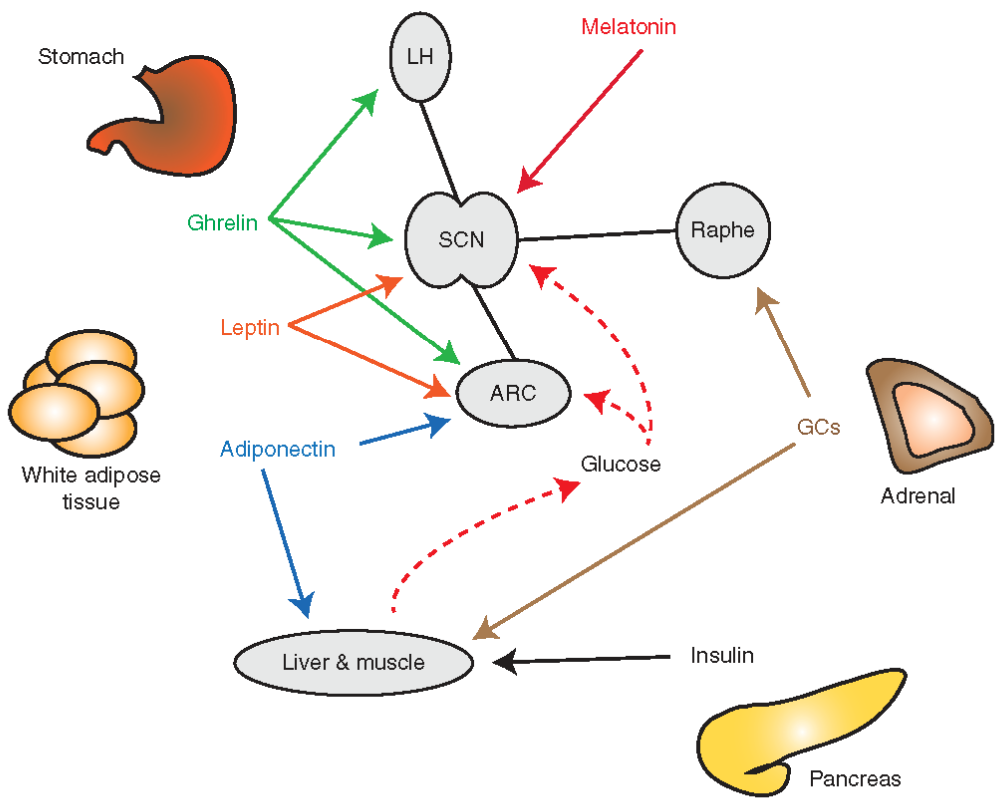

Figure 4

Endocrine feedback to the cIrcadlan clock. Varlous hormones can directly or indirectly feedback on central and peripheral clock function. In the brain endocrine targets with connections to the $\mathrm{SCN}$ include the orexinergic neurons of the lateral hypothalamus (LH), the arcuate nucleus (ARC), and

\section{Cortisol}

Exposure to jetlag or sleep perturbations (such as sleep restriction or shift work) results in a transient mismatch between the internal circadian time and the external lightdark cycle. Symptoms of jetlag include decreased alertness, motor coordination and cognitive performance, sleep disturbances, gastrointestinal disruption, and loss of appetite (Waterhouse et al. 2005). Sleep restriction and daytime sleep - hallmarks of a night shift work schedule are associated with increased BMI and risk of metabolic syndrome, and alterations in circulating endocrine parameters such as insulin, glucose, and GCs (Wu et al. 2008, Rehman etal. 2010, Baron et al. 2011). Cortisol rhythms are also affected by jet travel, even when only three or fewer time zones are crossed (Doane et al. 2010), as well as by relatively subtle advances in sleep timing (Dijk et al. 2012).

GC steroids secreted from the adrenal gland are integral regulators of energy metabolism as well as the response to immune challenge and stress. GC disruption is

\begin{tabular}{|lr}
\hline http://Ime.endocrinology-journals.org & 2014 Society for Endocrinology \\
DOI: $10.1530 / \mathrm{ME}-13-0118$ & Printed in Great Britain
\end{tabular}

the raphe nuclel of the bralnstem. Other endocrine effects may be mediated via peripheral tissues and clocks such as the liver and muscle. For details see text.

associated with a variety of disorders. Cushing's disease is characterized by excess cortisol, with symptoms including hypertension, hyperglycemia, sleep disorders, depression, and weight gain (Carroll \& Findling 2010). Addison's disease, characterized by a lack of cortisol, is accompanied by symptoms of weight loss, elevated sensitivity to stress, hypotension, mood disorders, and hypoglycemia (Mitchell \& Pearce 2012).

GCs have been shown to directly affect circadian clock gene expression in a number of tissues, such as white adipose tissue, liver, and kidney (Gomez-Abellan et al. 2012, Pezük et al. 2012). Adrenalectomy shortens reentrainment in the SCN, lung, and kidney following phase shifts, suggesting that GCs may serve to stabilize the phase of peripheral clocks against external noise (Pezük et al. 2012). In the case of jetlag-induced circadian desynchrony, it was shown that manipulation of the GC rhythm could speed up or slow down activity adaptation to the new light-dark schedule, depending on the intervention time (Kiessling et al. 2010). This study 


\begin{tabular}{|l|l|l|c|c|}
\hline Review & A H TSANG and others & Endocrine circadian rhythms & $\mathbf{5 2 : 1}$ & R9 \\
\hline
\end{tabular}

highlights the exciting therapeutic potential of GCs in the treatment of jetlag and other desynchrony disorders.

\section{Melatonin}

The best-studied physiological effect of melatonin is its modulatory function on sleep/wake cycle regulation in humans. Application of exogenous melatonin has been shown to decrease the latency to sleep, increase total sleep line, and pronole sleep maintenance (Sack t al. 1997, Sharkey et al. 2001). In contrast, blocking the nocturnal release of melatonin by suppressing the sympathetic innervation to the pineal results in increased total wake time (Van Den Heuvel et al. 1997). Moreover, exogenous melatonin can influence sleep macro architecture (Dijk et al. 1995, 1997). Because of its sleep-promoting effect, melatonin treatment is frequently used to ameliorate the symptoms of jetlag or to improve sleep quality during the daytime in night-shift workers (Aeschbach et al. 2009).

Beyond its effect on sleep, melatonin has been shown to directly signal to the SCN. In contrast to GRs (see above), high densities of MT1 and MT2 receptors in the SCN have been demonstrated (Gillette \& McArthur 1996). In rodents, timed daily administration of high concentrations of exogenows melatonin can entrain the free-running endogenous rhythm under constant darkness conditions (Armstrong et al. 1986, Redman \& Armstrong 1988). Similarly, timed application of melatonin can entrain blind human subjects (Arendt \& Broadway 1987, Sack et al. 2000). In vitro, melatonin application to cultured SCN explants affects amplitude and phase of the circadian rhythm of neuronal firing (Liu et al. 1997, Shimomura et al. 2010). The acute inhibitory effect of melatonin on neuronal activity seems to be mediated by MT1 receptor (Liu et al. 1997), while the phase-resetting effect relies on MT2 receptor signalling (Hunt et al. 2001). It is worthy of mention that melatonin is also capable of modulating the production of adrenal GCs. In humans and monkeys, acute melatonin administration suppresses the production of cortisol (Torres-Farfan et al. 2003, Campino et al. 2011). More recently, it has been demonstrated using foetal rats that timed melatonin application can entrain adrenal gland rhythms (Torres-Farfan et al. 2011). Thus, together melatonin and GC rhythms appear to stabilize circadian phase and precision for different physiological systems.

\section{Ghrelin and insulin}

The timing of food intake is an important entrainment signal for peripheral clocks, best characterized in, but not

\begin{tabular}{|lr}
\hline http://Ime.endocrinology-journals.org & 92014 Society for Endocrinology \\
DOI: $10.1530 / \mathrm{ME}-13-0118$ & Printed in Great Britain
\end{tabular}

limited to, the liver and adipose clocks (Stephan 2002). Anticipatory behavior just before scheduled feeding (food anticipatory activity (FAA)) is seen in animals with restricted access to food. This is characterized by increased activity and changes to body temperature, GC rhythms and hepatic P450 enzymatic function (Krieger et al. 1977, Hirao et al. 2006), which function to prepare the body for the anticipated food intake. When food access is confined to the normal rest period, these processes can uncouple peripheral oscillators from the central clock that stays locked to the light regimen. Ghrelin is secreted in anticipation of feeding, regardless of the light-dark cycle, from gastric oxyntic gland cells which possess a functional clock (LeSauter et al. 2009). Ghrelin stimulates appetite via its actions on the hypothalamic orexigenic peptides, neuropeptide $\mathrm{Y}$ and orexin, and on mesolimbic reward centres (Abizaid et al. 2006, Toshinai et al. 2006). In shift workers, the post-prandial ghrelin slump is attenuated, perhaps contributing to overeating (Schiavo-Cardozo et al. 2012). Ghrelin administration increases FAA; however, studies on rodents lacking functional ghrelin signalling are contradictory. Mice lacking ghrelin receptors are reported to have dampened FAA (LeSauter et al. 2009), whilst mice lacking preproghrelin show intact FAA responses during restricted feeding (RF; Szentirmai et al. 2010). Ghrelin can feed back onto the circadian clock by directly affecting clock gene expression in the SCN (Yannielli et al. 2007). In vivo studies show that ghrelin treatment increases food intake, but only shifts behavioral rhythms under fasted conditions (Yannielli et al. 2007).

Insulin represents another potential food-inducible clock synchronizer. Insulin secretion from pancreatic beta cells is clock-gated, and disruption of the positive arm of the clock - CLOCK or BMAL1 - results in hypoinsulinemia (Marcheva et al. 2010, Sadacca et al. 2011), while disruption of the clock's negative regulators - PERs and CRYs - is associated with hyperinsulinemia (Zhao et al. 2012, Barclay et al. 2013). Insulin sensitivity is reduced in shift workers, and accompanied by increased beta cell activity, suggesting a pre-diabetic state (Esquirol et al. 2012). But insulin can also feed back to the clock. Tahara et al. (2011) used daytime RF in mice to demonstrate insulin-dependent alterations of clock gene rhythms in the liver, and a similar response was seen in primarily cultured rat hepatocytes (Yamajuku et al. 2012). It would be remiss to discuss the effects of insulin on the clock without discussing the effects of glucose as a direct function of insulin signalling. Glucose can directly affect circadian gene expression in fibroblasts and the SCN (Hirota et al. 2002, Iwanaga et al. 2005). In the absence of

Published by Bioscientifica Ltd. 
insulin signalling, for example in diabetic rats, circadian clock phase is shifted in the heart, suggesting that high glucose levels can directly impinge on clock regulation (Young et al. 2002). Under RF conditions, sucrose (but not lipid) induces phase shifts and FAA (Stephan \& Davidson 1998).

The concept of food-inducible factors acting as powerful entertainers of the clock system is ratified by a number of studies which employ RF to rescue clock gene rhythms as well as physiological rhylhnns under desynchronous conditions. In a rat model of night work, restricting food intake to the normal activity phase restores glucose rhythms and prevents weight gain (Salgado-Delgado et al. 2010). In a study on a mouse model of shift work, restoring normal food intake rhythms concurrently restores clock gene rhythmicity in the liver, as well as triglyceride, glycerol and GC rhythms, and gluconeogenesis (Barclay et al. 2012). While these data suggest a direct link between peripheral clock regulation and energy homeostasis, the phase relationship between clock gene expression and the transcriptional activity of metabolismassociated genes is variable, suggesting an interplay between local and systemic factors (Reznick et al. 2013).

\section{Leptìn and adiponectin}

It is widely established that clock disruption results in metabolic perturbations, and ultimately obesity (reviewed in Froy (2010)). Conversely, high fat diet (HFD) can dampen clock gene rhythmicity in the liver and fat, and well as affecting behavioral rhythms (Kohsaka et al. 2007). HFD results in loss of diurnal feeding patterns in rodents, and subsequent alteration to GC, insulin, and glucose rhythms (Kohsaka et al. 2007). A study by Kaneko et al. (2009) showed altered clock gene expression the brainstem of mice fed with a HFD, as well as in genetically obese mice such as $o b / o b$ (lacking the leptin gene) and KK-A(y) mice (a spontaneous diabetic mouse model). However, arguably the most dramatic effects of HFD and obesity are the effects seen on circulating adipokines such as leptin and adiponectin.

Leptin is secreted from white adipose tissue in response to glucose stimulation, and signals via appetite centres in the hypothalamus to promote satiety and prevent excess energy consumption. Circulating leptin shows a diurnal rhythm, peaking in the night in humans. In the models of obesity, leptin resistance can occur and in the absence of leptin's anorexigenic effects, this is accompanied by overeating (reviewed in Gautron \& Elmquist (2011)). In humans, acute HFD feeding results

\begin{tabular}{|lr}
\hline http://Ime.endocrinology-journals.org & 92014 Society for Endocrinology \\
DOI: $10.1530 / \mathrm{ME}-13-0118$ & Printed in Great Britain
\end{tabular}

in lower 24-h leptin (Havel et al. 1999), whereas hyperleptinemia and changes in leptin rhythmicity are observed in obese subjects in accordance with increased fat mass (Considine et al. 1996, Rosenbaum et al. 1996, van Dielen et al. 2002). Despite having no direct effect on locomotor activity, leptin can induce PER expression in the SCN of female mice and potentiate the phase-shifting effects of light in these animals (Mendoza et al. 2011). Ex vivo, leptin stimulation can reset the phase of the SCN clock (Prosser \& Bergeron 2003).

Adiponectin possesses insulin-sensitizing and antiinflammatory properties (reviewed in Harwood (2012)). Circulating adiponectin levels inversely correlate with obesity and leptin levels, and weight loss results in increased adiponectin (Hu et al. 1996, Yang et al. 2001, Matsubara et al. 2002). Adiponectin secretion shows both ultradian and circadian rhythms, with a nadir in the early hours of the morning in healthy adults (Gavrila et al. 2003, Scheer et al. 2010). In rodents, adiponectin peaks in the end of the light phase (inactive phase) and its rhythm is shifted under HFD (Ríos-Lugo et al. 2010). Bullen and colleagues showed decreased adiponectin levels relative to fat mass following HFD in rodents (Barnea et al. 2006, Bullen et al. 2007). To assess the effect of adiponectin on the circadian clock, Hashinaga and colleagnes nsed KK-Ta mice, a polygenic model of metabolic syndrome with hypoadiponectinemia. These mice have a shorter activity period under constant conditions and dampened circadian locomotor rhythms with increased light-phase activity relative to controls. Clock gene rhythms are phase-advanced in the liver and skeletal muscle in these mice. The introduction of the human adiponectin transgene into the liver of these mice restores locomotor rhythmicity, as well as hepatic clock gene phase (Hashinaga et al. 2013). These studies strongly indicate that leptin, adiponectin, and maybe other adipokines may have direct effects on molecular clock function.

\section{Summary and outlook}

In summary, many components of the endocrine system show strong circadian rhythmicity in both rodents and humans. Some of these hormones, such as melatonin and cortisol, are involved in disseminating the SCN timing signal to other parts of the body. Endocrine rhythms respond to factors that compromise the clock function, such as HFD, obesity, jetlag, and sleep disruption. In turn, the endocrine system feeds back on central and peripheral clocks to adapt circadian rhythms to altered physiological state. Given the profound effects endocrine and circadian

Published by Bioscientifica Ltd. 
systems have on general well-being and the development of various disorders, this mutual interaction might provide new targets for pharmacological interventions at the systemic level. Recent studies have shown that resetting of GC signalling can affect clock resetting during jetlag (Kiessling et al. 2010) and with the recent discovery of drugs directly impinging on clock function (Hirota $e t$ al. 2010, Solt et al. 2012) it may be possible to rescue endocrine regulation under desynchrony conditions such as shift work.

Declaration of interest

The authors declare that there is no conflict of interest that could be perceived as prejudicing the impartiality of the review reported.

\section{Funding}

This work was supported by grants from the German Research Foundation (DFG) and the Volkswagen Foundation to $\mathrm{H} \mathrm{O}$ and a Göttingen Graduate School for Neurosciences, Biophysics, and Molecular Biosciences (GGNB) fellowship from the University of Göttingen to A H T.

\section{References}

Abe M, Herzog ED, Yamazaki S, Straume M, Tei H, Sakaki Y, Menaker M \& Block GD 2002 Circadian rhythms in isolated brain regions. Journal of Neuroscience 22 350-356.

Abizaid A, Liu Z-W, Andrews ZB, Shanabrough M, Borok E, Elsworth JD, Roth RH, Sleeman MW, Picciotto MR, Tschöp MH et al. 2006 Ghrelin modulates the activity and synaptic input organization of midbrain dopamine neurons while promoting appetite. Journal of Clinical Investigation 116 3229-3239. (doi:10.1172/JCI29867)

Abraham U, Granada AE, Westermark PO, Heine M, Kramer A \& Herzel H 2010 Coupling governs entrainment range of circadian clocks. Molecular Systems Biology 6 438. (doi:10.1038/msb.2010.92)

Aeschbach D, Sher L, Postolache TT, Matthews JR, Jackson MA \& Wehr TA 2003 A longer biological night in long sleepers than in short sleepers. Joumal of Clinical Endocrinology and Metabolism 88 26-30. (doi:10.1210/ jc. 2002-020827)

Aeschbach D, Lockyer BJ, Dijk DJ, Lockley SW, Nuwayser ES, Nichols LD \& Czeisler CA 2009 Use of transdermal melatonin delivery to improve sleep maintenance during daytime. Clinical Pharmacology and Therapeutics 86 378-382. (doi:10.1038/clpt.2009.109)

Akashi M \& Takumi T 2005 The orphan nuclear receptor ROR $\alpha$ regulates circadian transcription of the mammalian core-clock Bmal1. Nature Structural \& Molecular Biology 12 441-448. (doi:10.1038/ nsmb925)

Akerstedt T 2003 Shift work and disturbed sleep/wakefulness. Occupational Medicine 53 89-94. (doi:10.1093/occmed/kqg046)

Andrews RV \& Folk GE Jr 1964 Circadian metabolic patterns in cultured hamster adrenal glands. Comparative Biochemistry and Physiology 11 393-409. (doi:10.1016/0010-406X(64)90006-4)

Arendt J \& Broadway J 1987 Light and melatonin as zeitgebers in man. Chronobiology International 4 273-282. (doi:10.3109/ 07420528709078534)

Armstrong SM, Cassone VM, Chesworth MJ, Redman JR \& Short RV 1986 Synchronization of mammalian circadian rhythms by melatonin. Joumal of Neural Transmission. Supplementum 21 375-394.

http://me.endocrinology-journals.org
DOl: 10.1530/ME-13-0118

Balsalobre A, Damiola F \& Schibler U 1998 A serum shock induces circadian gene expression in mammalian tissue culture cells. Cell 93 929-937. (doi:10.1016/S0092-8674(00)81199-X)

Barclay JL, Husse J, Bode B, Naujokat N, Meyer-Kovac J, Schmid SM, Lehnert $\mathrm{H} \&$ Oster $\mathrm{H} 2012$ Circadian desynchrony promotes metabolic disruption in a mouse model of shiftwork. PLOS ONE 7 e37150. (doi:10.1371/journal.pone.0037150)

Barclay JL, Shostak A, Leliavski A, Tsang AH, Johren O, Muller-Fielitz H, Landgraf D, Naujokat N, van der Horst GT \& Oster H 2013 High fat diet-induced hyperinsulinemia and tissue-specific insulin resistance in Cry deficient mice. American Joumal of Physiology. Endocrinology and Metabolism 204 E1053-E1063. (doi:10.1152/ajpendo.00512.2012) Bamea M, Shanay A, Stark AH \& Madar Z 2006 A high-fat diet has a tissuespecific effect on adiponectin and related enzyme expression. Obesity 14 2145-2153. (doi:10.1038/oby.2006.251)

Baron KG, Reid KJ, Kern AS \& Zee PC 2011 Role of sleep timing in caloric intake and BMI. Obesity 19 1374-1381. (doi:10.1038/oby.2011.100)

Barrett P \& Bolborea M 2012 Molecular pathways involved in seasonal body weight and reproductive responses governed by melatonin. Journal of Pineal Research 52 376-388. (doi:10.1111/j.1600-079X.2011.00963.x)

Beccuti G \& Pannain S 2011 Sleep and obesity. Current Opinion in Clinical Nutrition and Metabolic Care 14 402-412. (doi:10.1097/MCO. 0b013e3283479109)

Buhr ED, Yoo SH \& Takahashi JS 2010 Temperature as a universal resetting cue for mammalian circadian oscillators. Science 330 379-385. (doi:10.1126/science.1195262)

Buijs RM, Wortel J, Van Heerikhuize JJ, Feenstra MG, Ter Horst GJ, Romijn HJ \& Kalsbeek A 1999 Anatomical and functional demonstration of a multisynaptic suprachiasmatic nucleus adrenal (cortex) pathway. European Joumal of Neuroscience 11 1535-1544. (doi:10.1046/ j.1460-9568.1999.00575.x

Buijs RM, van Eden CG, Goncharuk VD \& Kalsbeek A 2003 The biological clock tunes the organs of the body: timing by hormones and the autonomic nervous system. Joumal of Endocrinology 177 17-26. (doi:10.1677/joe.0.1770017)

Bullen JW Jr, Bluher S, Kelesidis T \& Mantzoros CS 2007 Regulation of adiponectin and its receptors in response to development of dietinduced obesity in mice. American Joumal of Physiology. Endocrinology and Metabolism 292 E1079-E1086. (doi:10.1152/ajpendo.00245.2006)

Cain SW, Dennison CF, Zeitzer JM, Guzik AM, Khalsa SB, Santhi N, Schoen MW, Czeisler CA \& Duffy JF 2010 Sex differences in phase angle of entrainment and melatonin amplitude in humans. Journal of Biological Rhythms 25 288-296. (doi:10.1177/0748730410374943)

Campino C, Valenzuela FJ, Torres-Farfan C, Reynolds HE, Abarzua-Catalan L, Arteaga E, Trucco C, Guzman S, Valenzuela GJ \& Seron-Ferre M 2011 Melatonin exerts direct inhibitory actions on ACTH responses in the human adrenal gland. Hormone and Metabolic Research 43 337-342. (doi:10.1055/s-0031-1271693)

Carroll T \& Findling J 2010 The diagnosis of Cushing's syndrome. Reviews in Endocrine \& Metabolic Disorders 11 147-153. (doi:10.1007/s11154-0109143-3)

Chen W \& Baler R 2000 The rat arylalkylamine N-acetyltransferase E-box: differential use in a master vs. a slave oscillator. Brain Research. Molecular Brain Research 81 43-50. (doi:10.1016/S0169-328X(00)00160-1)

Cheng MY, Bullock CM, Li C, Lee AG, Bermak JC, Belluzzi J, Weaver DR, Leslie FM \& Zhou QY 2002 Prokineticin 2 transmits the behavioural circadian rhythm of the suprachiasmatic nucleus. Nature $\mathbf{4 1 7} 405-410$. (doi: $10.1038 / 417405$ a)

Christ E, Pfeffer M, Korf HW \& von Gall C 2010 Pineal melatonin synthesis is altered in Period1 deficient mice. Neuroscience 171 398-406. (doi:10.1016/j.neuroscience.2010.09.009)

Considine RV, Sinha MK, Heiman ML, Kriauciunas A, Stephens TW, Nyce MR, Ohannesian JP, Marco CC, McKee LJ, Bauer TL et al. 1996 Serum immunoreactive-leptin concentrations in normal-weight and obese humans. New England Journal of Medicine 334 292-295. (doi:10.1056/NEJM199602013340503) 
Cowell IG 2002 E4BP4/NFIL3, a PAR-related bZIP factor with many roles. BioEssays 24 1023-1029. (doi:10.1002/bies.10176)

Dibner C, Schibler U \& Albrecht U 2010 The mammalian circadian timing system: organization and coordination of central and peripheral clocks. Annual Review of Physiology 72 517-549. (doi:10.1146/annurevphysiol-021909-135821)

van Dielen FM, van 't Veer C, Buurman WA \& Greve JW 2002 Leptin and soluble leptin receptor levels in obese and weight-losing individuals. Joumal of Clinical Endocrinology and Metabolism 87 1708-1716. (doi:10. $1210 /$ jc.87.4.1708)

Dijk DJ, Roth C, Landolt HP, Werth E, Aeppli M, Achermann P \& Borbely AA 1995 Melatonin effect on daytime sleep in men: suppression of EEG low frequency activity and enhancement of spindle frequency activity. Neuroscience Letters 201 13-16. (doi:10.1016/0304-3940(95)12118-N)

Dijk DJ, Shanahan TL, Duffy JF, Ronda JM \& Czeisler CA 1997 Variation of electroencephalographic activity during non-rapid eye movement and rapid eye movement sleep with phase of circadian melatonin rhythm in humans. Joumal of Physiology 505 851-858. (doi:10.1111/j.14697793.1997.851ba.x)

Dijk DJ, Duffy JF, Riel E, Shanahan TL \& Czeisler CA 1999 Ageing and the circadian and homeostatic regulation of human sleep during forced desynchrony of rest, melatonin and temperature rhythms. Journal of Physiology 516 611-627. (doi:10.1111/j.1469-7793.1999.0611v.x)

Dijk DJ, Duffy JF, Silva EJ, Shanahan TL, Boivin DB \& Czeisler CA 2012 Amplitude reduction and phase shifts of melatonin, cortisol and other circadian rhythms after a gradual advance of sleep and light exposure in humans. PLOS ONE 7 e30037. (doi:10.1371/journal.pone.0030037)

Doane LD, Kremen WS, Eaves LJ, Eisen SA, Hauger R, Hellhammer D, Levine S, Lupien S, Lyons MJ, Mendoza Set al. 2010 Associations between jet lag and cortisol diurnal rhythms after domestic travel. Health Psychology 29 117-123. (doi:10.1037/a0017865)

Drijfhout WJ, van der Linde AG, Kooi SE, Grol CJ \& Westerink BH 1996 Norepinephrine release in the rat pineal gland: the input from the biological clock measured by in vivo microdialysis. Joumal of Neurochemistry 66 748-755. (doi:10.1046/j.1471-4159.1996.66020748.x)

Edwards AV \& Jones CT 1993 Autonomic control of adrenal function. Joumal of Anatomy 183 291-307.

Esquirol Y, Bongard V, Ferrieres J, Verdier $\mathrm{H}$ \& Perret B 2012 Shiftwork and higher pancreatic secretion: early detection of an intermediate state of insulin resistance? Chronobiology International 29 1258-1266. (doi: 10.3109/07420528.2012.719959)

Foster RG \& Soni BG 1998 Extraretinal photoreceptors and their regulation of temporal physiology. Reviews of Reproduction 3 145-150. (doi:10 $1530 /$ ror. 0.0030145 )

Froy $\mathrm{O} 2010$ Metabolism and circadian rhythms - implications for obesity. Endocrine Reviews 31 1-24. (doi:10.1210/er.2009-0014)

Gaston S \& Menaker M 1968 Pineal function: the biological clock in the sparrow? Science 160 1125-1127. (doi:10.1126/science.160.3832.1125)

Gautron L \& Elmquist JK 2011 Sixteen years and counting: an update on leptin in energy balance. Joumal of Clinicallnvestigation 121 2087-2093. (doi:10.1172/JCI45888)

Gavrila A, Peng C-K, Chan JL, Mietus JE, Goldberger AL \& Mantzoros CS 2003 Diurnal and ultradian dynamics of serum adiponectin in healthy men: comparison with leptin, circulating soluble leptin receptor, and cortisol patterns. Joumal of Clinical Endocrinology and Metabolism $\mathbf{8 8}$ 2838-2843. (doi:10.1210/jc.2002-021721)

Gillette MU \& McArthur AJ 1996 Circadian actions of melatonin at the suprachiasmatic nucleus. Behavioral Brain Research 73 135-139. (doi:10.1016/0166-4328(96)00085-X)

Girotti M, Weinberg MS \& Spencer RL 2009 Diurnal expression of functional and clock-related genes throughout the rat HPA axis: system-wide shifts in response to a restricted feeding schedule. American Joumal of Physiology. Endocrinology and Metabolism 296 E888-E897. (doi:10.1152/ajpendo.90946.2008)

Gomez-Abellan P, Diez-Noguera A, Madrid JA, Lujan JA, Ordovas JM \& Garaulet M 2012 Glucocorticoids affect $24 \mathrm{~h}$ clock genes expression in

http://me.endocrinology-journals.org
DOl: 10.1530/ME-13-0118

human adipose tissue explant cultures. PLOS ONE 7 e50435. (doi:10.1371/journal.pone.0050435)

Gooley JJ, Chamberlain K, Smith KA, Khalsa SB, Rajaratnam SM, Van Reen E, Zeitzer JM, Czeisler CA \& Lockley SW 2011 Exposure to room light before bedtime suppresses melatonin onset and shortens melatonin duration in humans. Joumal of Clinical Endocrinology and Metabolism 96 E463-E472. (doi:10.1210/jc.2010-2098)

Goto M, Oshima I, Tomita T \& Ebihara S 1989 Melatonin content of the pineal gland in different mouse strains. Joumal of Pineal Research 7 195-204. (doi:10.1111/j.1600-079X.1989.tb00667.x)

Green DJ \& Gillette R 1982 Circadian rhythm of firing rate recorded from single cells in the rat suprachiasmatic brain slice. Brain Research $\mathbf{2 4 5}$ 198-200. (do1:10.1016/0006-8993(82)90361-4)

Groos G \& Hendriks J 1982 Circadian rhythms in electrical discharge of rat suprachiasmatic neurones recorded in vitro. Neuroscience Letters $\mathbf{3 4}$ 283-288. (doi:10.1016/0304-3940(82)90189-6)

Guilding C \& Piggins HD 2007 Challenging the omnipotence of the suprachiasmatic timekeeper: are circadian oscillators present throughout the mammalian brain? European Journal of Neuroscience 25 3195-3216. (doi:10.1111/j.1460-9568.2007.05581.x)

Guilding C, Hughes AT, Brown TM, Namvar S \& Piggins HD 2009 A riot of rhythms: neuronal and glial circadian oscillators in the mediobasal hypothalamus. Molecular Brain 2 28. (doi:10.1186/1756-6606-2-28)

el-Haji Fuleihan G, Klerman EB, Brown EN, Choe Y, Brown EM \& Czeisler CA 1997 The parathyroid hormone circadian rhythm is truly endogenous - a general clinical research center study. Joumal of Clinical Endocrinology and Metabolism 82 281-286. (doi:10.1210/jc.82.1.281)

Hankins MW, Peirson SN \& Foster RG 2008 Melanopsin: an exciting photopigment. Trends in Neurosciences 31 27-36. (doi:10.1016/j.tins. 2007.11.002)

Harwood HJ Jr 2012 The adipocyte as an endocrine organ in the regulation of metabolic homeostasis. Neuropharmacology 63 57-75. (doi:10.1016/ j.neuropharm.2011.12.010)

Hashinaga T, Wada N, Otabe S, Yuan X, Kurita Y, Kakino S, Tanaka K, Sato T, Kojima M, Ohki T et al. 2013 Modulation by adiponectin of circadian clock rhythmicity in model mice for metabolic syndrome. Endocrine Joumal 60 483-492. (doi:10.1507/endocri.EJ12-0305)

Havel PJ, Townsend R, Chaump L \& Teff K 1999 High-fat meals reduce 24-h circulating leptin concentrations in women. Diabetes 48 334-341. (doi:10.2337/diabetes.48.2.334)

Hendrickson AE, Wagoner N\& Cowan WM 1972 An autoradiographic and electron microscopic study of retino-hypothalamic connections. Zeitschrift für Zellforschung und Mikroskopische Anatomie 135 1-26. Zeitschrift für Zellforschung und

Henley DE, Leendertz JA, Russell GM, Wood SA, Taheri S, Woltersdorf WW \& Lightman SL 2009 Development of an automated blood sampling system for use in humans. Joumal of Medical Engineering \& Technology 33 199-208. (doi: $10.1080 / 03091900802185970$ )

Hirao J, Arakawa S, Watanabe K, Ito K \& Furukawa T 2006 Effects of restricted feeding on daily fluctuations of hepatic functions including $\mathrm{p} 450$ monooxygenase activities in rats. Joumal of Biological Chemistry 281 3165-3171. (doi:10.1074/jbc.M511194200)

Hirota T, Okano T, Kokame K, Shirotani-Ikejima H, Miyata T \& Fukada Y 2002 Glucose down-regulates Per 1 and Per 2 mRNA levels and induces circadian gene expression in cultured Rat-1 fibroblasts. Journal of Biological Chemistry 277 44244-44251. (doi:10.1074/jbc.M206233200) Hirota T, Lee JW, Lewis WG, Zhang EE, Breton G, Liu X, Garcia M, Peters EC, Etchegaray JP, Traver D et al. 2010 High-throughput chemical screen identifies a novel potent modulator of cellular circadian rhythms and reveals CKI $\alpha$ as a Clock regulatory kinase. PLoS Biology 8 e 1000559 (doi:10.1371/journal.pbio.1000559)

Hu E, Liang P \& Spiegelman BM 1996 AdipoQ is a novel adipose-specific gene dysregulated in obesity. Joumal of Biological Chemistry 271 10697-10703. (doi:10.1074/jbc.271.18.10697)

Hunt AE, Al-Ghoul WM, Gillette MU \& Dubocovich ML 2001 Activation of $\mathrm{MT}_{2}$ melatonin receptors in rat suprachiasmatic nucleus phase 
advances the circadian clock. American Joumal of Physiology. Cell Physiology 280 C110-C118.

Ishida A, Mutoh T, Ueyama T, Bando $\mathrm{H}$, Masubuchi S, Nakahara D, Tsujimoto G \& Okamura H 2005 Light activates the adrenal gland: timing of gene expression and glucocorticoid release. Cell Metabolism 2 297-307. (doi:10.1016/j.cmet.2005.09.009)

Iwanaga $H$, Yano M, Miki H, Okada K, Azama T, Takiguchi S, Fujiwara Y, Yasuda T, Nakayama M, Kobayashi M et al. 2005 Per2 gene expressions in the suprachiasmatic nucleus and liver differentially respond to nutrition factors in rats. Journal of Parenteral and Enteral Nutrition 29 157-161. (doi:10.1177/0148607105029003157)

Kalsbeek A, Garidou ML, Palm IF, Van Der Vliet J, Simonneaux V, Pevet P \& Buljs RM 2000 Melatonnin sees the light: blocking GABA-eryic transmission in the paraventricular nucleus induces daytime secretion of melatonin. European Joumal of Neuroscience 12 3146-3154. (doi: $10.1046 / j .1460-9568.2000 .00202 . x$ )

Kaneko K, Yamada T, Tsukita S, Takahashi K, Ishigaki Y, Oka Y \& Katagiri H 2009 Obesity alters circadian expressions of molecular clock genes in the brainstem. Brain Research 1263 58-68. (doi:10.1016/j.brainres. 2008.12.071)

Kennaway DJ, Voultsios A, Varcoe TJ \& Moyer RW 2003 Melatonin and activity rhythm responses to light pulses in mice with the Clock mutation. American Journal of Physiology. Regulatory, Integrative and Comparative Physiology 284 R1231-R1240. (doi:10.1152/ajpregu.00697. 2002)

Kennaway DJ, Owens JA, Voultsios A \& Varcoe TJ 2006 Functional central rhythmicity and light entrainment, but not liver and muscle rhythmicity, are Clock independent. American Joumal of Physiology. Regulatory, Integrative and Comparative Physiology 291 R1172-R1180 (doi:10.1152/ajpregu.00223.2006)

Kiessling S, Eichele G \& Oster H 2010 Adrenal glucocorticoids have a key role in circadian resynchronization in a mouse model of jet lag. Joumal of Clinical Investigation 12026002609 . (doi:10.1172 JCI41192)

Klein DC \& Moore RY 1979 Pineal N-acetyltransferase and hydroxyindoleO-methyltransferase: control by the retinohypothalamic tract and the suprachiasmatic nucleus. Brain Research 174 245-262. (doi:10.1016/ $0006-8993(79) 90848-5)$

Kleitman N 1970 Study wakefulness. Study the rest-activity cycle. Don't just study sleep. International Psychiatry Clinics 7 381-384.

Kohsaka A, Laposky AD, Ramsey KM, Estrada C, Joshu C, Kobayashi Y, Turek FW \& Bass J 2007 High-fat diet disrupts behavioral and molecular circadian rhythms in mice. Cell Metabolism 6 414-421. (doi:10.1016/ i.cmet.2007.09.006)

Konopka RJ \& Benzer S 1971 Clock mutants of Drosophila melanogaster. PNAS 68 2112-2116. (doi:10.1073/pnas.68.9.2112)

Kramer A, Yang FC, Snodgrass P, Li X, Scammell TE, Davis FC \& Weitz CJ 2001 Regulation of daily locomotor activity and sleep by hypothalamic EGF receptor signaling. Science 294 2511-2515. (doi:10.1126/science. 1067716)

Kraves S\& Weitz CJ 2006 A role for cardiotrophin-like cytokine in the circadian control of mammalian locomotor activity. Nature Neuroscience 9 212-219. (doi: $10.1038 / \mathrm{nn} 1633$ )

Krieger DT, Hauser H \& Krey LC 1977 Suprachiasmatic nuclear lesions do not abolish food-shifted circadian adrenal and temperature rhythmicity. Science 197 398-399. (doi:10.1126/science.877566)

Kwon S \& Hermayer KL 2013 Glucocorticoid-induced hyperglycemia. American Joumal of the Medical Sciences 345 274-277. (doi:10.1097/MAJ. 0b013e31828a6a01)

Larsen PJ, Enquist LW \& Card JP 1998 Characterization of the multisynaptic neuronal control of the rat pineal gland using viral transneuronal tracing. European Joumal of Neuroscience 10 128-145. (doi:10.1046/j.1460-9568.1998.00003.x)

Lee C, Etchegaray JP, Cagampang FR, Loudon AS \& Reppert SM 2001 Posttranslational mechanisms regulate the mammalian circadian clock. Cell 107 855-867. (doi:10.1016/S0092-8674(01)00610-9)
LeSauter J, Hoque N, Weintraub M, Pfaff DW \& Silver R 2009 Stomach ghrelin-secreting cells as food-entrainable circadian clocks. PNAS 106 13582-13587. (doi:10.1073/pnas.0906426106)

Li X, Sankrithi N \& Davis FC 2002 Transforming growth factor- $\alpha$ is expressed in astrocytes of the suprachiasmatic nucleus in hamster: role of glial cells in circadian clocks. Neuroreport 13 2143-2147. (doi:10.1097/00001756-200211150-00031)

Liu C, Weaver DR, Jin X, Shearman LP, Pieschl RL, Gribkoff VK \& Reppert SM 1997 Molecular dissection of two distinct actions of melatonin on the suprachiasmatic circadian clock. Neuron 19 91-102. (doi:10.1016/ S0896-6273(00)80350-5)

Liu AC, Welsh DK, Ko CH, Tran HG, Zhang EE, Priest AA, Buhr ED, Singer O, Meeker $\mathrm{K}$, Verina IM et al. 2007 Intercellular coupling confers robustness against mutations in the $\mathrm{SCN}$ circadian clock network. Cell 129 605-616. (doi:10.1016/j.cell.2007.02.047)

Liu AC, Tran HG, Zhang EE, Priest AA, Welsh DK \& Kay SA 2008 Redundant function of REV-ERB $\alpha$ and $\beta$ and non-essential role for Bmal1 cycling in transcriptional regulation of intracellular circadian rhythms. PLoS Genetics 4 e1000023. (doi:10.1371/journal.pgen.1000023)

Luiten PG, ter Horst GJ \& Steffens AB 1987 The hypothalamus, intrinsic connections and outflow pathways to the endocrine system in relation to the control of feeding and metabolism. Progress in Neurobiology $\mathbf{2 8}$ 1-54. (doi: 10.1016/0301-0082(87)90004-9)

Marcheva B, Ramsey KM, Buhr ED, Kobayashi $\mathrm{Y}$, Su H, Ko CH, Ivanova G, Omura C, Mo S, Vitaterna MH et al. 2010 Disruption of the clock components CLOCK and BMAL1 leads to hypoinsulinaemia and diabetes. Nature 466 627-631. (doi:10.1038/nature09253)

Matsubara M, Maruoka S \& Katayose S 2002 Inverse relationship between plasma adiponectin and leptin concentrations in normal-weight and obese women. European Joumal of Endocrinology 147 173-180. (doi:10.1530/eje.0.1470173)

McNamara P, Seo SB, Rudic RD, Sehgal A, Chakravarti D \& FitzGerald GA 2001 Regulation of CLOCK and MOP1 by nuclear hormone receptors in the vasculature: a humoral mechanism to reset a peripheral clock. Cell 105 877-889. (doi:10.1016/S0092-8674(01)00401-9)

Menaker M, Moreira LF \& Tosini G 1997 Evolution of circadian organization in vertebrates. Brazilian Journal of Medical and Biological Research 30 305-313. (doi:10.1590/S0100-879X1997000300003)

Mendoza J, Lopez-Lopez C, Revel FG, Jeanneau K, Delerue F, Prinssen E, Challet E, Moreau JL \& Grundschober C 2011 Dimorphic effects of leptin on the circadian and hypocretinergic systems of mice. Journal of Neuroendocrinology 23 28-38. (doi:10.1111/j.1365-2826.2010.02072.x)

Migeon CJ, Tyler FH, Mahoney JP, Florentin AA, Castle H, Bliss EL \& Samuels LT 1956 The diurnal variation of plasma levels and urinary excretion on 17-hydroxycorticosteroids in normal subjects, night workers and blind subjects. Journal of Clinical Endocrinology and Metabolism 16 622-633. (doi:10.1210/jcem-16-5-622)

Miller WL \& Bose HS 2011 Early steps in steroidogenesis: intracellular cholesterol trafficking. Joumal of Lipid Research 52 2111-2135. (doi:10.1194/jlr.R016675)

Mills JN, Minors DS \& Waterhouse JM 1978 Adaptation to abrupt time shifts of the oscillator(s) controlling human circadian rhythms. Ioumal of Physiology 285 455-470.

Mitchell AL \& Pearce SHS 2012 Autoimmune Addison disease: pathophysiology and genetic complexity. Nature Reviews. Endocrinology 8 306-316. (doi: $10.1038 /$ nrendo.2011.245)

Moore RY 1996 Neural control of the pineal gland. Behavioral Brain Research 73 125-130. (doi:10.1016/0166-4328(96)00083-6)

Moore RY \& Eichler VB 1972 Loss of a circadian adrenal corticosterone rhythm following suprachiasmatic lesions in the rat. Brain Research 42 201-206. (doi:10.1016/0006-8993(72)90054-6)

Moore RY \& Lenn NJ 1972 A retinohypothalamic projection in the rat. Joumal of Comparative Neurology 146 1-14. (doi:10.1002/cne. 901460102)

Nagoshi E, Saini C, Bauer C, Laroche T, Naef F \& Schibler U 2004 Circadian gene expression in individual fibroblasts: cell-autonomous

Published by Bioscientifica Ltd. 
and self-sustained oscillators pass time to daughter cells. Cell 119 693-705. (doi:10.1016/j.cell.2004.11.015)

Nduhirabandi F, du Toit EF \& Lochner A 2012 Melatonin and the metabolic syndrome: a tool for effective therapy in obesity-associated abnormalities? Acta Physiologica 205 209-223. (doi:10.1111/j.17481716.2012.02410.x)

Ohayon MM, Lemoine P, Arnaud-Briant V \& Dreyfus M 2002 Prevalence and consequences of sleep disorders in a shift worker population. Joumal of Psychosomatic Research $\mathbf{5 3}$ 577-583. (doi:10.1016/S00223999(02)00438-5)

Okamura H 2007 Suprachiasmatic nucleus clock time in the mammalian circadian system. Cold Spring Harbor Symposia on Quantitative Biology $\mathbf{7 2}$ 551-556. (doi:10.1101/sqb.2007.72.033)

Oster H, Damerow S, Hut RA \& Eichele G $2006 a$ Transcriptional profiling in the adrenal gland reveals circadian regulation of hormone biosynthesis genes and nucleosome assembly genes. Journal of Biological Rhythms 21 350-361. (doi:10.1177/0748730406293053)

Oster H, Damerow S, Kiessling S, Jakubcakova V, Abraham D, Tian J, Hoffmann MW \& Eichele G $2006 \mathrm{~b}$ The circadian rhythm of glucocorticoids is regulated by a gating mechanism residing in the adrenal cortical clock. Cell Metabolism 4 163-173. (doi:10.1016/j.cmet. 2006.07.002)

Ottenweller JE \& Meier AH 1982 Adrenal innervation may be an extrapituitary mechanism able to regulate adrenocortical rhythmicity in rats. Endocrinology 111 1334-1338. (doi:10.1210/endo-111-4-1334)

Ottenweller JE, Meier AH, Ferrell BR, Horseman ND \& Proctor A 1978 Extrapituitary regulation of the circadian rhythm of plasma corticosteroid concentration in rats. Endocrinology 103 1875-1879. (doi:10.1210/endo-103-5-1875)

Perreau-Lenz S, Kalsbeek A, Pevet P \& Buijs RM 2004 Glutamatergic clock output stimulates melatonin synthesis at night. European Joumal of Neuroscience 19 318-324. (doi:10.1111/j.0953-816X.2003.03132.X)

Pezük P, Mohawk JA, Wang LA \& Menaker M 2012 Glucocorticoids as entraining signals for peripheral circadian oscillators. Endocrinology 153 4775-4783. (doi:10.1210/en.2012-1486)

Phillipson OT \& Griffiths AC 1985 The topographic order of inputs to nucleus accumbens in the rat. Neuroscience 16 275-296. (doi:10.1016/ 0306-4522(85)90002-8)

Pincus G, Romanoff LP \& Carlo J 1954 The excretion of urinary steroids by men and women of various ages. Joumal of Gerontology 9 113-132. (doi:10.1093/geronj/9.2.113)

Preitner N, Damiola F, Lopez-Molina L, Zakany J, Duboule D, Albrecht U \& Schibler U 2002 The orphan nuclear receptor REV-ERB $\alpha$ controls circadian transcription within the positive limb of the mammalian circadian oscillator. Cell 110 251-260. (doi:10.1016/S0092$8674(02) 00825-5)$

Prosser RA \& Bergeron HE 2003 Leptin phase-advances the rat suprachiasmatic circadian clock in vitro. Neuroscience Letters $\mathbf{3 3 6}$ 139-142. (doi:10.1016/\$0304-3940(02)01234-X)

Quay WB 1970 Physiological significance of the pineal during adaptation to shifts in photoperiod. Physiology \& Behavior 5 353-360. (doi:10.1016/ 0031-9384(70)90110-1)

Quay WB 1972 Pineal homeostatic regulation of shifts in the circadian activity rhythm during maturation and aging. Transactions of the New York Academy of Sciences 34 239-254. (doi:10.1111/j.2164-0947. 1972.tb02679.x)

Ralph CL, Mull D, Lynch HJ \& Hedlund L 1971 A melatonin rhythm persists in rat pineals in darkness. Endocrinology 89 1361-1366. (doi: $10.1210 /$ endo-89-6-1361)

Ralph MR, Foster RG, Davis FC \& Menaker M 1990 Transplanted suprachiasmatic nucleus determines circadian period. Science $\mathbf{2 4 7}$ 975-978. (doi:10.1126/science.2305266)

Redman JR \& Armstrong SM 1988 Reentrainment of rat circadian activity rhythms: effects of melatonin. Joumal of Pineal Research 5 203-215. (doi:10.1111/j.1600-079X.1988.tb00782.x)

http://]me.endocrinology-journals.org DOI: 10.1530/MME-13-0118
Rehman JU, Brismar K, Holmback U, Akerstedt T \& Axelsson J 2010 Sleeping during the day: effects on the 24 -h patterns of IGF-binding protein 1, insulin, glucose, cortisol, and growth hormone. European Journal of Endocrinology 163 383-390. (doi:10.1530/EJE-10-0297)

Reick M, Garcia JA, Dudley C \& McKnight SL 2001 NPAS2: an analog of clock operative in the mammalian forebrain. Science 293 506-509. (doi:10.1126/science.1060699)

Reppert SM, Perlow MJ, Ungerleider LG, Mishkin M, Tamarkin L, Orloff DG, Hoffman HJ \& Klein DC 1981 Effects of damage to the suprachiasmatic area of the anterior hypothalamus on the daily melatonin and cortisol rhythms in the rhesus monkey. Journal of Neuroscience 1 1414-1425.

Reznick J, Preston E, Wilks DL, Beale SM, Turner N \& Cooney GJ 2013 Altered feeding differentially regulates circadian rhythms and energy metabolism in liver and muscle of rats. Biochimica et Biophysica Acta 1832 228-238. (doi:10.1016/j.bbadis.2012.08.010)

Ríos-Lugo MJ, Cano P, Jiménez-Ortega V, Fernández-Mateos MP, Scacch PA, Cardinali DP \& Esquifino AI 2010 Melatonin effect on plasma adiponectin, leptin, insulin, glucose, triglycerides and cholesterol in normal and high fat-fed rats. Joumal of Pineal Research 49 342-348. (doi:10.1111/j.1600-079X.2010.00798.X)

Ripperger JA \& Schibler U 2006 Rhythmic CLOCK-BMAL1 binding to multiple E-box motifs drives circadian Dbp transcription and chromatin transitions. Nature Genetics 38 369-374. (doi:10.1038/ng1738)

Ripperger JA, Shearman LP, Reppert SM \& Schibler U 2000 CLOCK, an essential pacemaker component, controls expression of the circadian transcription factor DBP. Genes and Development 14 679-689. (doi:10.1101/gad.14.6.679)

Roseboom PH, Namboodiri MA, Zimonjic DB, Popescu NC, Rodriguez IR, Gastel JA \& Klein DC 1998 Natural melatonin 'knockdown' in C57BL/6J mice: rare mechanism truncates serotonin $\mathrm{N}$-acetyltransferase. Brain Research. Molecular Brain Research 63 189-197. (doi:10.1016/S0169$328 \times(98) 00273-3)$

Rosenbaum M, Nicolson M, Hirsch J, Heymsfield SB, Gallagher D, Chu F \& Leibel RL 1996 Effects of gender, body composition, and menopause on plasma concentrations of leptin. Joumal of Clinical Endocrinology and Metabolism 81 3424-3427. (doi:10.1210/jc.81.9.3424)

Sack RL, Hughes RJ, Edgar DM \& Lewy AJ 1997 Sleep-promoting effects of melatonin: at what dose, in whom, under what conditions, and by what mechanisms? Sleep 20 908-915.

Sack RL, Brandes RW, Kendall AR \& Lewy AJ 2000 Entrainment of free-running circadian rhythms by melatonin in blind people. New England Journal of Medicine 343 1070-1077. (doi:10.1056/NEJM200010123431503)

Sadacca LA, Lamia KA, deLemos AS, Blum B \& Weitz CJ 2011 An intrinsic circadian clock of the pancreas is required for normal insulin release and glucose homeostasis in mice. Diabetologia 54 120-124. (doi:10.1007/s00125-010-1920-8)

Saeb-Parsy K, Lombardelli S, Khan FZ, McDowall K, Au-Yong IT \& Dyball RE 2000 Neural connections of hypothalamic neuroendocrine nuclei in the rat. Joumal of Neuroendocrinology 12 635-648. (doi:10.1046/j.13652826.2000.00503.X)

Salgado-Delgado R, Angeles-Castellanos M, Saderi N, Buijs RM \& Escobar C 2010 Food intake during the normal activity phase prevents obesity and circadian desynchrony in a rat model of night work. Endocrinology 151 1019-1029. (doi:10.1210/en.2009-0864)

Sandi C 2011 Glucocorticoids act on glutamatergic pathways to affect memory processes. Trends in Neurosciences 34 165-176. (doi:10.1016/ j.tins.2011.01.006)

Sato TK, Panda S, Miraglia LJ, Reyes TM, Rudic RD, McNamara P, Naik KA, FitzGerald GA, Kay SA \& Hogenesch JB 2004 A functional genomics strategy reveals Rora as a component of the mammalian circadian clock. Neuron 43 527-537. (doi:10.1016/j.neuron.2004.07.018)

Scheer FA, Hilton MF, Mantzoros CS \& Shea SA 2009 Adverse metabolic and cardiovascular consequences of circadian misalignment. PNAS 106 4453-4458. (doi:10.1073/pnas.0808180106)

Scheer FA, Chan JL, Fargnoli J, Chamberland J, Arampatzi K, Shea SA, Blackburn GL \& Mantzoros CS 2010 Day/night variations of 
high-molecular-weight adiponectin and lipocalin-2 in healthy men studied under fed and fasted conditions. Diabetologia 53 2401-2405. (doi:10.1007/s00125-010-1869-7)

Schiavo-Cardozo D, Lima MM, Pareja JC \& Geloneze B 2012 Appetiteregulating hormones from the upper gut: disrupted control of xenin and ghrelin in night workers. Clinical Endocrinology [in press]. (doi: $10.1111 /$ cen.12114)

Sharkey KM, Fogg LF \& Eastman CI 2001 Effects of melatonin administration on daytime sleep after simulated night shift work. Joumal of Sleep Research 10 181-192. (doi:10.1046/j.1365-2869.2001.00256.x)

Shibata S, Oomura Y, Kita H \& Hattori K 1982 Circadian rhythmic changes of neuronal activity in the suprachiasmatic nucleus of the rat hypothalamic sllce. Brain Research 247 154-158. (do1:10.1016/00068993(82)91041-1)

Shimomura K, Lowrey PL, Vitaterna MH, Buhr ED, Kumar V, Hanna P, Omura C, Izumo M, Low SS, Barrett RK et al. 2010 Genetic suppression of the circadian Clock mutation by the melatonin biosynthesis pathway. PNAS 107 8399-8403. (doi:10.1073/pnas.1004368107)

Silver R, LeSauter J, Tresco PA \& Lehman MN 1996 A diffusible coupling signal from the transplanted suprachiasmatic nucleus controlling circadian locomotor rhythms. Nature 382 810-813. (doi:10.1038/ 382810a0)

Silverman MN \& Sternberg EM 2012 Glucocorticoid regulation of inflammation and its functional correlates: from HPA axis to glucocorticoid receptor dysfunction. Annals of the New York Academy of Sciences 1261 55-63. (doi:10.1111/j.1749-6632.2012.06633.x)

Solt LA, Wang Y, Banerjee S, Hughes T, Kojetin DJ, Lundasen T, Shin Y, Liu J, Cameron MD, Noel R et al. 2012 Regulation of circadian behaviour and metabolism by synthetic REV-ERB agonists. Nature $\mathbf{4 8 5}$ 62-68. (doi:10.1038/nature11030)

Son GH, Chung S, Choe HK, Kim HD, Baik SM, Lee H, Lee HW, Choi S, Sun W, Kim $\mathrm{H}$ et al. 2008 Adrenal peripheral clock controls the autonomous circadian thythm of glucocorticoid by causing rhythmic steroid production. PNAS 105 20970-20975. (doi:10.1073/pnas.0806962106)

Sousa-Pinto A \& Castro-Correia J 1970 Light microscopic observations on the possible retinohypothalamic projection in the rat. Experimental Brain Research 11 515-527. (doi:10.1007/BF00233972)

Srinivasan V, Pandi-Perumal SR, Brzezinski A, Bhatnagar KP \& Cardinali DP 2011 Melatonin, immune function and cancer. Recent Patents on Endocrine, Metabolic \& Immune Drug Discovery 5 109-123. (doi:10.2174/ 187221411799015408)

Srinivasan V, De Berardis D, Shillcutt SD \& Brzezinski A 2012 Role of melatonin in mood disorders and the antidepressant effects of agomelatine. Expert Opinion on Investigational Drugs 21 1503-1522. (doi:10.1517/13543784.2012.711314)

Stephan FK 2002 The "other" circadian system: food as a Zeitgeber. Joumal of Biological Rhythms 17 284-292. (doi:10.1177/074873040201700402)

Stephan FK \& Zucker I 1972 Circadian rhythms in drinking behavior and locomotor activity of rats are eliminated by hypothalamic lesions. PNAS 69 1583-1586. (doi:10.1073/pnas.69.6.1583)

Stephan FK \& Davidson AJ 1998 Glucose, but not fat, phase shifts the feeding-entrained circadian clock. Physiology \& Behavior 65 277-288. (doi:10.1016/S0031-9384(98)00166-8)

Szentirmai E, Kapas L, Sun Y, Smith RG \& Krueger JM 2010 Restricted feeding-induced sleep, activity, and body temperature changes in normal and preproghrelin-deficient mice. American Joumal of Physiology. Regulatory, Integrative and Comparative Physiology 298 R467-R477. (doi:10.1152/ajpregu.00557.2009)

Tahara Y, Otsuka M, Fuse Y, Hirao A \& Shibata S 2011 Refeeding after fasting elicits insulin-dependent regulation of Per 2 and Rev-erb $\alpha$ with shifts in the liver clock. Joumal of Biological Rhythms 26 230-240. (doi:10.1177/0748730411405958)

Teclemariam-Mesbah R, Ter Horst GJ, Postema F, Wortel J \& Buijs RM 1999 Anatomical demonstration of the suprachiasmatic nucleus-pineal pathway. Joumal of Comparative Neurology 406 171-182. (doi:10.1002 (SICI) 1096-9861 (19990405) 406:2 < 171::AID-CNE3>3.0.CO;2-U)
Torres-Farfan C, Richter HG, Rojas-Garcia P, Vergara M, Forcelledo ML, Valladares LE, Torrealba F, Valenzuela GJ \& Seron-Ferre M $2003 \mathrm{mt} 1$ Melatonin receptor in the primate adrenal gland: inhibition of adrenocorticotropin-stimulated cortisol production by melatonin. Joumal of Clinical Endocrinology and Metabolism 88 450-458. (doi:10. $1210 / \mathrm{jc} .2002-021048$ )

Torres-Farfan C, Mendez N, Abarzua-Catalan L, Vilches N, Valenzuela GJ \& Seron-Ferre M $2011 \mathrm{~A}$ circadian clock entrained by melatonin is ticking in the rat fetal adrenal. Endocrinology 152 1891-1900. (doi:10.1210/en. 2010-1260)

Toshinai K, Yamaguchi H, Sun Y, Smith RG, Yamanaka A, Sakurai T, Date Y, Mondal MS, Shimbara T, Kawagoe T et al. 2006 Des-acyl ghrelin induces food intake by a mechanisin independent of the growth hormone secretagogue receptor. Endocrinology 147 2306-2314. (doi:10.1210/en. 2005-1357)

Tosini G \& Menaker M 1998 Multioscillatory circadian organization in a vertebrate, Iguana iguana. Journal of Neuroscience 18 1105-1114.

Ueda HR, Chen W, Adachi A, Wakamatsu H, Hayashi S, Takasugi T, Nagano M, Nakahama K, Suzuki Y, Sugano S et al. 2002 A transcription factor response element for gene expression during circadian night. Nature 418 534-539. (doi:10.1038/nature00906)

Van Den Heuvel CJ, Reid KJ \& Dawson D 1997 Effect of atenolol on nocturnal sleep and temperature in young men: reversal by pharmacological doses of melatonin. Physiology \& Behavior 61 795-802. (doi:10.1016/S0031-9384(96)00534-3)

Vitaterna MH, King DP, Chang AM, Kornhauser JM, Lowrey PL, McDonald JD, Dove WF, Pinto LH, Turek FW \& Takahashi JS 1994 Mutagenesis and mapping of a mouse gene, Clock, essential for circadian behavior Science 264 719-725. (doi:10.1126/science.8171325)

Vivien-Roels B, Malan A, Rettori MC, Delagrange P, Jeanniot JP \& Pevet P 1998 Daily variations in pineal melatonin concentrations in inbred and outbred mice. Joumal of Biological Rhythms 13 403-409. (doi:10.1177) $071873098129000228)$

Waterhouse J, Nevill A, Finnegan J, Williams P, Edwards B, Kao SY \& Reilly T 2005 Further assessments of the relationship between jet lag and some of its symptoms. Chronobiology Intemational 22 121-136. (doi:10.1081/CBI-200036909)

Watts AG \& Swanson LW 1987 Efferent projections of the suprachiasmatic nucleus: II. Studies using retrograde transport of fluorescent dyes and simultaneous peptide immunohistochemistry in the rat. Joumal of Comparative Neurology 258 230-252. (doi:10.1002/cne.902580205)

Watts AG, Swanson LW \& Sanchez-Watts G 1987 Efferent projections of the suprachiasmatic nucleus: I. Studies using anterograde transport of Phaseolus vulgaris leucoagglutinin in the rat. Journal of Comparative Neurology 258 204-229. (doi:10.1002/cne.902580204)

Watts AG, Tanimura $S \&$ Sanchez-Watts G 2004 Corticotropin-releasing hormone and arginine vasopressin gene transcription in the hypothalamic paraventricular nucleus of unstressed rats: daily rhythms and their interactions with corticosterone. Endocrinology $145529-540$ (doi:10.1210/en.2003-0394)

Wehr TA, Aeschbach D \& Duncan WC Jr 2001 Evidence for a biological dawn and dusk in the human circadian timing system. Journal of Physiology 535 937-951. (doi:10.1111/j.1469-7793.2001.t01-1-00937.x)

Welsh DK, Logothetis DE, Meister M \& Reppert SM 1995 Individual neurons dissociated from rat suprachiasmatic nucleus express independently phased circadian firing rhythms. Neuron 14 697-706. (doi:10.1016/0896-6273(95)90214-7)

Wu H, Zhao Z, Stone WS, Huang L, Zhuang J, He B, Zhang P \& Li Y 2008 Effects of sleep restriction periods on serum cortisol levels in healthy men. Brain Research Bulletin 77 241-245. (doi:10.1016/j.brainresbull. 2008.07.013)

Yamajuku D, Inagaki T, Haruma T, Okubo S, Kataoka Y, Kobayashi S, Ikegami K, Laurent T, Kojima T, Noutomi K et al. 2012 Real-time monitoring in three-dimensional hepatocytes reveals that insulin acts as a synchronizer for liver clock. Scientific Reports 2 439. (doi:10.1038/ srep00439)

Published by Bioscientifica Ltd. 
Yamanaka Y, Suzuki Y, Todo T, Honma K \& Honma S 2010 Loss of circadian rhythm and light-induced suppression of pineal melatonin levels in Cryl and Cry2 double-deficient mice. Genes to Cells 15 1063-1071. (doi:10.1111/j.1365-2443.2010.01443.x)

Yamazaki S, Numano R, Abe M, Hida A, Takahashi R, Ueda M, Block GD, Sakaki $Y$, Menaker $M \&$ Tei $H 2000$ Resetting central and peripheral circadian oscillators in transgenic rats. Science $\mathbf{2 8 8} 682-685$. (doi: $10.1126 /$ science.288.5466.682)

Yang W-S, Lee W-J, Funahashi T, Tanaka S, Matsuzawa Y, Chao C-L, Chen C-L, Tai T-Y \& Chuang L-M 2001 Weight reduction increases plasma levels of an adipose-derived anti-inflammatory protein, adiponectin. Joumal of Clinical Endocrinology and Metabolism 86 3815-3819. (doi.10.1210/jc.86.8.3815)

Yannielli PC, Molyneux PC, Harrington ME \& Golombek DA 2007 Ghrelin effects on the circadian system of mice. Journal of Neuroscience $\mathbf{2 7}$ 2890-2895. (doi:10.1523/JNEUROSCI.3913-06.2007)
Yoo SH, Yamazaki S, Lowrey PL, Shimomura K, Ko CH, Buhr ED, Siepka SM, Hong HK, Oh WJ, Yoo OJ et al. 2004 PERIOD2::LUCIFERASE real-time reporting of circadian dynamics reveals persistent circadian oscillations in mouse peripheral tissues. PNAS 101 5339-5346. (doi:10.1073/pnas. 0308709101)

Young ME, Wilson CR, Razeghi P, Guthrie PH \& Taegtmeyer $\mathrm{H} 2002$ Alterations of the circadian clock in the heart by streptozotocininduced diabetes. Joumal of Molecular and Cellular Cardiology 34 223-231. (doi:10.1006/jmcc.2001.1504)

Zhang EE \& Kay SA 2010 Clocks not winding down: unravelling circadian networks. Nature Reviews. Molecular Cell Biology 11 764-776. (doi: 10.1038/nrm2995)

Zhao $Y$, Zhang Y, Zhou M, Wang S, IIua Z \& Zhang J 2012 Loss of mPer2 increases plasma insulin levels by enhanced glucose-stimulated insulin secretion and impaired insulin clearance in mice. FEBS Letters $\mathbf{5 8 6}$ 1306-1311. (doi:10.1016/j.febslet.2012.03.034)

Received in final form 22 August 2013

Accepted 29 August 2013

Accepted Preprint published online 30 August 2013 


\title{
Tissue-Specific Interaction of Per1/2 and Dec2 in the Regulation of Fibroblast Circadian Rhythms
}

\author{
Anthony H. Tsang, ${ }^{* 1}$ Carmen Sánchez-Moreno, ${ }^{\dagger, 1}$ Brid Bode, ${ }^{*}$ Moritz J. Rossner, ${ }^{\ddagger}$ \\ Marta Garaulet, ${ }^{\dagger}$ and Henrik Oster ${ }^{*}, 2$ \\ ${ }^{*}$ Circadian Rhythms Group, Göttingen, Germany, 'Department of Physiology, Faculty of Biology, \\ University of Murcia, Spain, ${ }^{\ddagger}$ Max Planck Institute for Experimental Medicine, Göttingen, Germany, \\ and ${ }^{\$}$ Medical Department I, University of Lübeck, Germany
}

\begin{abstract}
In mammals, the molecular circadian clockwork is comprised of interlocked transcriptional-translational feedback loops (TTLs). Three Period (Per1-3) and $2 \operatorname{Dec}(\operatorname{Dec} 1 / 2)$ genes interact in regulating the activity of the transcriptional activators CLOCK/NPAS2 and BMAL1. While deletion of Per1 and Per2 in mice results in behavioral arrhythmicity, Dec deletion has less dramatic effects on activity rhythms, affecting primarily phase of entrainment and freerunning period. In intact animals, clock gene mutant phenotypes are often masked due to intercellular coupling mechanisms that stabilize cellular rhythms. Therefore, to study Per/Dec genetic interaction at the cellular level, we isolated fibroblasts from different tissues of Per1, Per2, and Dec2 single and double mutant mice. We show that in the cellular TTL, Pers and Dec2 act in a principally synergistic way, but tissue-specific differences in this interaction are seen. A rescue of rhythmicity in Yer' 2 mutant cells atter additional deletion of Dec2 was observed, indicating that in the absence of Per2, DEC2 destabilizes TTL function. Rhythm power in Per1/Dec2 and Per2/Dec2 double mutants was strongly reduced, suggesting that interaction of Dec2 with both Per genes is important for stabilizing clock period. Contrary to what was observed for behavior, nonsynergistic effects of Dec 2 and Per1/2 mutations were observed on cellular clock phase regulation that do not correlate with period effects. Our data reveal cell type-specific interactions of Per1/2 and Dec2 in the regulation of period, phase, and rhythm sustainment, emphasizing the differential organization of the mammalian clock machinery in different tissues.
\end{abstract}

Key words Per1, Per2, Dec2, circadian clock, cellular rhythms, fibroblasts, luciferase

In most organisms, endogenous timekeeping systems have evolved that organize physiological and behavioral adaptation to the 24-h day (Brown et al., 2012). In mammals, these so-called circadian clocks are based on cellular oscillators driven by a set of clock genes organized in a system of interlocked transcriptional-translational feedback loops (TTLs) (Oster, 2006; Takahashi et al., 2008). Positive TTL

1. These authors contributed equally to the study.

2. To whom all correspondence should be addressed: Henrik Oster, Circadian Rhythms Group, Max Planck Institute for Biophysical Chemistry, Am Fassberg 11, 37077 Göttingen, Germany; e-mail: henrik.oster@mpibpc.mpg.de.

JOURNAL OF BIOLOGICAL RHYTHMS, Vol. 27 No. 6, December 2012 478-489

DOI: $10.1177 / 0748730412462838$

(C) 2012 The Author(s)

478 
elements include the transcription factors CLOCK, NPAS2, and BMAL1 (ARNTL) that drive expression of negative elements such as Period (Per1-3) and Cryptochrome (Cry1/2) genes via E-box regulatory promoter elements during the day (Bunger et al., 2000; Gekakis et al., 1998). In the night, PER/CRY protein complexes translocate into the nucleus where they interfere with CLOCK/NPAS2/BMAL1, thereby inhibiting their own transcription (Griffin et al., 1999; Kume et al., 1999; Sun et al., 1997). Toward morning, successive degradation of PER/CRY proteins releases the inhibitory pressure on CLOCK/NPAS2/BMAL1, and a new round of E-box-mediated transcription resumes (Lee et al., 2001; Yoshitane et al., 2009). The periodicity of this feedback is believed to be regulated by posttranslational mechanisms and accessory loops that stabilize the necessary delay between production and inhibitory action of negative TTL elements. One such loop involves the transcriptional modulator DEC2 (SHARP-1, BHLH-42) (Honma et al., 2002). Dec2 transcription is controlled by CLOCK/ NPAS2/BMAL1 (Hamaguchi et al., 2004; Noshiro et al., 2005), and DEC2 proteins can-depending on time and tissue - act as either activators or inhibitors of E-box transcription (Bode et al., 2011b; Rossner et al., 2008). Very similar characteristics have been observed for the PER proteins (Preitner et al., 2002; Zheng et al., 2001). In previous experiments we have shown that $\mathrm{Per}$ and Dec genes interact closely and in a timeof-day dependent way in regulating activity rhythms and clock gene expression in the mammalian circadian pacemaker of the suprachiasmatic nucleus (SCN) (Bode et al., 2011a, 2011b).

Unlike other tissues, the SCN is characterized by tightly coupled neurons generating a highly stabilized rhythm at the tissue level (Aton et al., 2005; Liu et al., 2007; Liu and Reppert, 2000; Maywood et al., 2006; Yamaguchi et al., 2003). Previous studies have shown that by this coupling, SCN cells become highly resistant to the destabilizing effects of clock gene mutations (Jakubcakova et al., 2007; Ti11 et al., 2007; Pando et al., 2002; Robles et al., 2010). Moreover, the organization of the clock machinery and, thus, the phenotype of specific clock gene mutations can differ between different tissues (Cermakian et al., 2001; DeBruyne et al., 2007b; Jakubcakova et al., 2007; Nakashima et al., 2008; Reick et al., 2001; Robles et al., 2010).

To analyze the interactivity of PER and DEC feedback in molecular clock regulation at the cellular level and in different tissues, we here compared molecular rhythms of primary fibroblasts from ear and lung tissue preparations of Per1, Per2, and Dec2 single and double mutant mice. Our data suggest a close, cell type-specific interaction of Per1/2 and Dec2 in the regulation of period, phasing, and sustainment of molecular circadian rhythms, emphasizing the multiform organization of the mammalian clock machinery in different tissues.

\section{MATERIALS AND METHODS}

\section{Primary Cell Cultures}

Mouse ear and lung fibroblasts were obtained from adult male and female Per1 mice (Per1 ${ }^{\text {m1Brd }}$ ) (Zheng et al., 2001), Per2 mice (Per2 ${ }^{\text {tm } 1 \text { Brd }}$ ) (Zheng et al., 1999), Dec2 mutant mice (Bhlhe41 $1^{\text {miMrio }}$ ) (Rossner et al., 2008), and PER2::LUC mice (Per2 ${ }^{\text {tm Ift }}$ ) (Yoo et al., 2004) on a C57B/6J background. Six or 7 independent preparations were done per genotype spread over several days, and always 2 or more genotypes were processed in parallel to minimize unspecific effects due to experimental variations. Tissues were extracted, rinsed with ice-cold HBSS, and subjected to mincing and subsequent enzymatic digestion. Ear tissues ( 2 cars per preparation) were digested with $100 \mu \mathrm{g} /$ mL Liberase TM (Roche Applied Science, Mannheim, Germany) in the presence of $20 \% \mathrm{FBS}$ at $37^{\circ} \mathrm{C}$ and $5 \%$ $\mathrm{CO}_{2}$ overnight. Lung tissues (2 lung wings per preparation) were digested with $100 \mu \mathrm{g} / \mathrm{mL}$ Liberase TM in the presence of $1 \% \mathrm{BSA}$ at $37^{\circ} \mathrm{C}$ with constant agitation for $1 \mathrm{~h}$. Dissociated cells were rinsed with PBS and plated onto $60-\mathrm{mm}$ cell culture dishes containing culture medium (DMEM with 20\% FBS, $100 \mathrm{U} / \mathrm{mL}$ penicillin, $0.1 \mathrm{mg} / \mathrm{mL}$ streptomycin, $2 \mathrm{mM}$ stable glutamine, $2.5 \mu \mathrm{g} / \mathrm{mL}$ amphotericine B) with medium change every second day. Upon confluence, fibroblast cells were trypsinized, and $5 \times 10^{5}$ cells were seeded to a $35-\mathrm{mm}$ cell culture dish containing culture medium as described above, but without amphotericine B. Viral transduction was conducted on the next day with $\sim 1.68 \times 10^{8}$ infection units (IFUs) per dish in the presence of $8 \mu \mathrm{g} / \mathrm{mL}$ polybrene. One day after transduction, cells were rinsed with PBS and restored with fresh culture medium.

\section{Bioluminescence Recording}

Cells were synchronized by $2 \mathrm{~h}$ of incubation with $100 \mathrm{nM}$ dexamethasone. After that, cells were changed to recording medium (same as above, but without phenol-red, plus $20 \mathrm{mM}$ HEPES, $1 \mathrm{mM}$ pyruvate, 
$100 \mu$ M D-luciferin; Biosynth AG, Staad, Switzerland). Dishes were sealed with silicon grease and cover glasses. Luminescence was measured using a LumiCycle luminometer (Actimetrics, Evanston, IL) at $32.5^{\circ} \mathrm{C}$. To assess dexamethasone sensitivity, earand lung-derived fibroblasts of PER2::LUC mice were isolated and plated into a 96-well plates with recording medium. Forty-eight hours after plating, plates were sealed with transparent film, and luminescence was recorded at $34^{\circ} \mathrm{C}$ using a Berthold TriStar LB 941 (Berthold Technologies, Wildbach, Germany). Luminescence was measured over $1 \mathrm{~h}$ to determine reference baselines. After that, cells were treated with various concentrations of dexamethasone, and recording was resumed for additional 24 hours. Sensitivity was determined by dividing the 24-h peak value of each trace by the respective pretreatment baseline.

\section{Lentivirus Production}

Bmall:luc encoding pBluF-puro plasmid was a kind gift from Prof. Steven Brown, University of Zurich, Switzerland. To produce Bmal1:luc lentiviral particles, each 10-cm dish of HEK293T cells was cotransfected with $10 \mu \mathrm{g} p s P 1 X 2$ (Addgene plasmid \#12260, Prof. Didier Trono, EPFL, Switzerland), $5 \mu \mathrm{g}$ pMD2.G (Addgene plasmid \#12259, Prof. Didier Trono, EPFL, Switzerland), and $15 \mu \mathrm{g} p$ BluF-puro using Xfect transfection reagent (Clontech, Saint-Germain-en-Laye, France). Cells were rinsed with PBS on the second day and restored with fresh culture medium. Viral containing medium was harvested at $36 \mathrm{~h}$ after transfection and stored at $4{ }^{\circ} \mathrm{C}$. Cells were restored with fresh medium, and further medium collections were performed on the next day. Two collections were pooled and concentrated using LentiX concentrator reagent (Clontech) according to the manufacturer's protocol. Virus titers were determined by transducing HEK $293 \mathrm{~T}$ cells with a serial dilution of GFPencoding lentiviral particles (produced as described above but with pWPI (Addgene plasmid \#12254, Prof. Didier Trono, EPFL, Switzerland) instead of pBluF-puro). At $72 \mathrm{~h}$ after transduction, GFP-positive cells were counted under a fluorescence microscope. The same serial dilution was also subjected to realtime quantitative PCR ( $\mathrm{qPCR}$ ) analysis using a primer pair amplifying the viral WPRE element (forward: 5'-GGCACTGACAATTCCGTGGT-3'; reverse: 5'-AGGGACGTAGCAGAAGGACG-3') to determine viral genome abundance. $\mathrm{qPCR}$ was also done for Bmal1-luc virus-containing medium, and the titer was estimated by comparison with the $p W P I$ abundance versus GFP calibration curve.

\section{Gene Reporter Assays}

Dbp:luc plasmid was a kind gift from Prof. Ueli Schibler, University of Geneva, Switzerland. HEK $293 \mathrm{~T}$ cells were plated into 96-well plates coated with polyD-lysine and transfected with the following expression plasmids: $10 \mathrm{ng}$ Dbp-luc and $2 \mathrm{ng} C M V$-Renilla luciferase with various combinations ( $20 \mathrm{ng}$ each) of the following clock gene constructs: HA-Clock, HA-Bmal1, HA-Dec1, FLAG-Dec2, Per1-GFP, Per2-V5, and pcDNA3.1 (mock transfection). We used Lipofectamine LTX transfection reagent (Life Technologies, Darmstadt, Germany). Forty-eight hours after transfection, luciferase activity was measured using Dual-Glo Luciferase Assay System (Promega, Mannheim, Germany) using the Berthold TriStar.

\section{Statistics}

For statistical comparisons, 6 or 7 independent fibroblast preparations for each tissue and genotype were used. Rhythmicity and period length were assessed by $\chi^{2}$ periodogram analysis over the first 3 days on 24-h moving average baseline-subtracted luminescence recordings. Dampening times (D) were determined by fitting a dampened sine wave to baseline-subtracted luminescence recordings over 5 days using a Levenberg-Marquardt algorithm on the following equation: $Y(t)=$ Amplitude ${ }^{*} \sin (2 * \pi$ ${ }^{*}$ Frequency ${ }^{*} t+$ Phase $) * e^{-(t / D)}+$ Offset. Dampening times are expressed relative to rhythm period length $(1 \mathrm{CT}=(2 * \pi *$ Frequency $) / 24 \mathrm{~h})$. To determine phase, the first peak of the baseline-subtracted luminescence curve was measured relative to the time of synchronization. All measurements were done using the Lumicycle analysis software (Actimetrics, Evanston, IL). Group statistics were done with GraphPad Prism 5 (GraphPad, La Jolla, CA).

\section{RESULTS}

Rhythmic Bmal1:luc Expression
in Per/Dec-Deficient Fibroblasts

We isolated and cultured primary fibroblasts of ear and lung tissues from adult wild-type (WT) and homozygous Per1 (P1), Per2 (P2), Dec2 (D2), Per1/ Dec2 (P1D2), and Per2/Dec2 (P2D2) single and double 
mutant mice. We chose 2 similar cell types from 2 different organs as this would allow us to compare tissuespecific effects under comparable culture conditions. At $60 \%$ confluence, cells were infected with Bmall:luc lentivirus. Transduction efficiency was determined on parallel infections using a GFP-expressing lentivirus. More than $95 \%$ of cells were expressing the transgene in all tested preparations (Suppl. Fig. S1). Upon confluency, cells were synchronized by dexamethasone treatment and transferred into a luminometer for luciferase activity measurements. Cells of all genotypes and tissues showed at least 2 full cycles of circadian rhythmicity, with the exception of P2 (both tissues) and P1D2 (lung). Representative luminescence recordings and corresponding normalizations are shown in Figure 1A for ear and Figure $1 \mathrm{~B}$ for lung preparations. To exclude that a difference in sensitivity to dexamethasone treatment might underlie the observed differences in rhythmicity in the 2 cell types, we stimulated PER2::LUC fibroblasts from both tissues with increasing amounts of dexamethasone and monitored the direct luciferase response over the next 24 hours. Luciferase activity was dose-dependently and similarly induced by dexamethasone in both tissues (Suppl Fig. S2).

Tissue-Specific Period Regulation in Per/DecDeficient Fibroblasts

Period length was shortened in $P 1$ relative to WT cells from both tissues (Fig. 2A, B). D2 cellular rhythms were significantly shortened in ear-derived (Fig. 2A) but not in lung-derived fibroblasts (Fig. 2B). P1D2 ear fibroblasts showed short periods comparable to those seen in P1 single mutant cells (Fig. 2A), indicating that period length is affected less by Dec2 than by Per1 in this tissue. In contrast in lung cells, arrhythmicity was observed after simultaneous mutation of Per1 and Dec2 (Fig. 2B). Surprisingly, circadian rhythmicity was rescued in Per2 mutant fibroblast preparations after additional deletion of

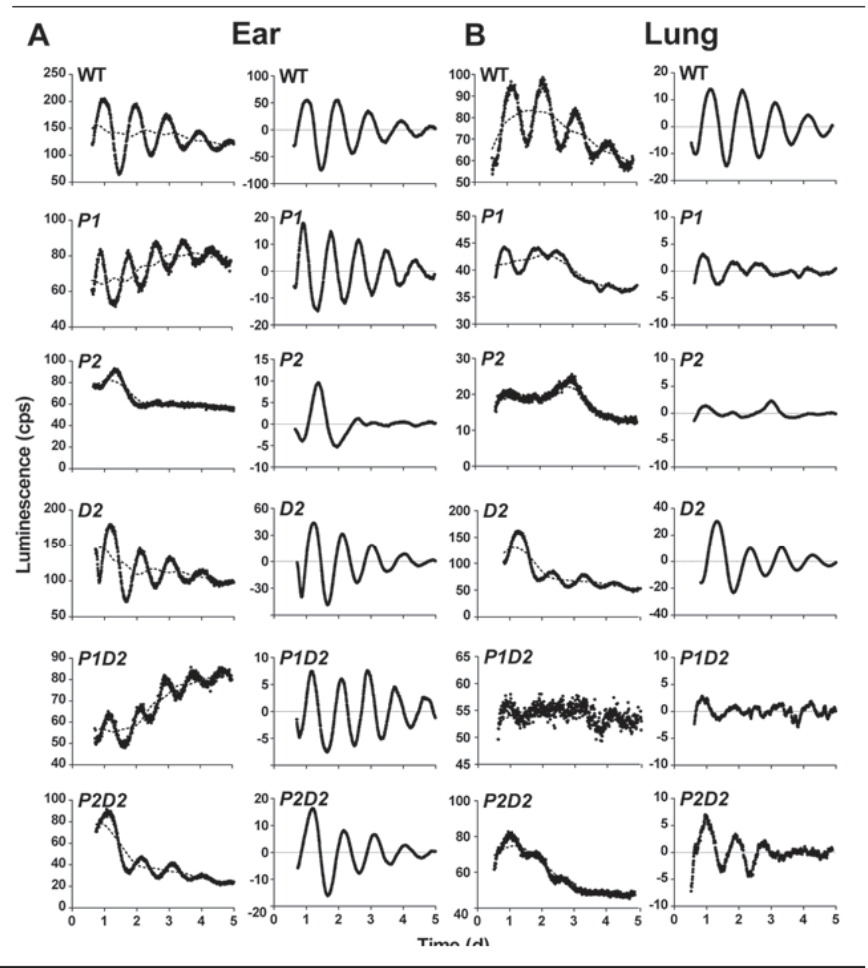

Figure 1. Cellular circadian rhythms in ear- and lung-derived PerlDec2 single and double mutant fibroblasts. (A, B) Representative luminescence recordings from wild(W1), Per1 (P1), Per2 (P2), Dec2 (D2) single, and Per1/Dec2 (P1D2) and Per2/Dec2 2) do uble mutant fibroblasts derived from ad ult mo use ear (A) and lung tissue (B) Left panels show raw data (black circles) and 24-h moving average baselines (dotted lines). Right panels show the same curves after baseline subtraction.

Dec2 (P2D2). While P2D2 ear fibroblasts showed short periods comparable to those of $D 2$ cells (Fig. $2 A$ ), in lung-derived $P 2 D 2$ cells periods were shorter than those of either WT or D2 single mutant fibroblasts (Fig. 2B). Comparable results were obtained when sine wave fits were used instead of periodogram analyses (Suppl. Fig. S3).

Tissue-Specific Regulation of Dampening Time and Rhythm Strength in Per/Dec-Deficient Fibroblasts

The restoration of circadian molecular rhythmicity in $P 2 D 2$ double mutant cells was very reminiscent of the rescue in behavioral locomotor rhythmicity observed in P2D2 double mutant animals (Bode et al., 2011a). To further assess interactive effects of Per1, Per2, and Dec2 on cellular rhythmicity, we quantified Bmal1:luc activity rhythm sustainment by determining 


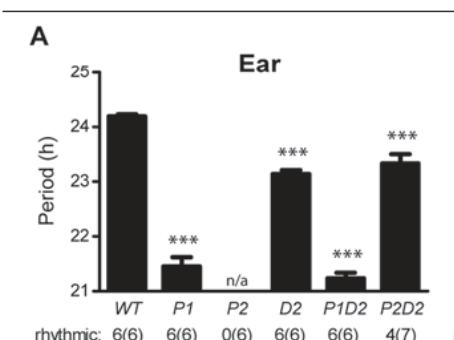

$\begin{array}{llllll}\text { rhythmic: } 6(6) & 6(6) & 0(6) & 6(6) & 6(6) & 4(7)\end{array}$

C

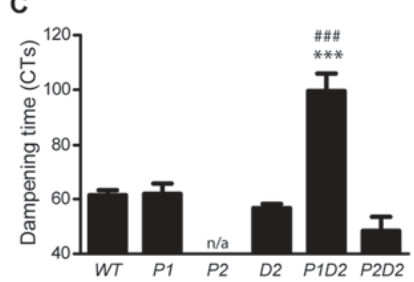

E

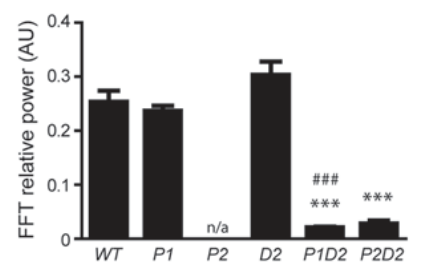

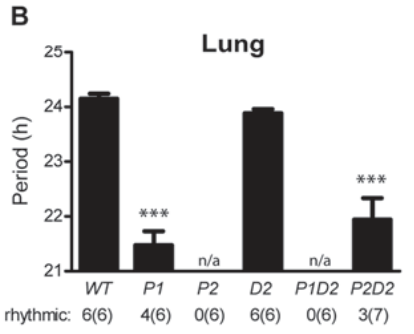

D

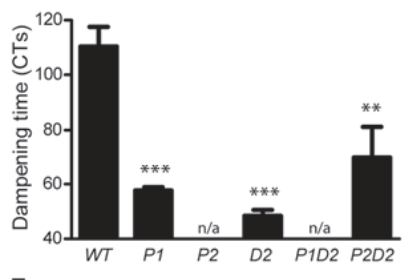

F

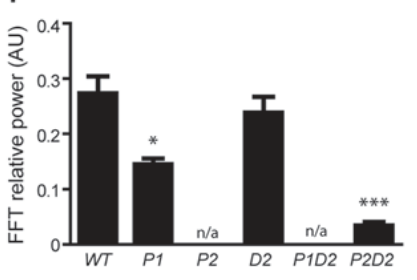

Figure 2. Period length and rhythm dampening in ear- and lung-derived PerlDec2 single and double mutant fibroblasts. (A, B) Period length as determined by $x^{2}$ periodogram analysis on baseline-subtracted recordings from ear-derived (A) and lung-derived cells (B). Values below depict numbers of rhythmic and total (in parentheses) preparations. (C, D) Dampening times as determined by dampened sine wave fitting on baseline-subtracted recordings from ear-derived $(C)$ and lung-derived cells (D). Dampening times were normalized to internal period to allow a better comparison of rhythm stability between cells with different internal periods. (E, F) Fast Fourier transformation (FFT) power analyses of luciferase activity during the first 3 days of the recordings from ear-derived (E) and lung-derived cells (F). All data are presented as means \pm SEM $(n=6-7) ;{ }^{*} p<0.05,{ }^{* * * \ldots+m} p<0.001$, 1-way ANOVA with Bonferroni posttest vs. WT $\left({ }^{*}\right)$ or P1 $\left({ }^{*}\right) ; n / a=$ no rhythm detectable.

dampening times and rhythm stability after fitting a dampened sine wave to baseline-subtracted luminescence recordings. For ear tissue-derived fibroblasts, dampening times were unaltered in $P 1, D 2$, and $P 2 D 2$ while, surprisingly, an increase in amplitude stability was observed in P1D2 cells (Fig. 2C). In contrast, in lung-derived cells, rhythm sustainment was strongly and consistently decreased in $P 1, D 2$, and P2D2 compared with WT (Fig. 2D). Rhythm stability values correlated well with dampening times (Fig. 2E, F), with faster dampening correlating with reduced rhythm power. Notable exceptions were seen in ear-derived P1D2 and lung-derived D2 cells.
In ear P1D2 cells, reduced rhythm power rates suggested that the extended amplitude sustainment was compromised by low period stability, making the oscillation rather unreliable. At the same time, in D2 lung fibroblasts, short dampening times were combined with relatively high rhythm power, indicating that early rhythm cessation may not derive from unstable period regulation per se.

\section{Tissue-Specific Regulation of Circadian Phase in Per/Dec- Deficient Fibroblasts}

One of the most prominent interactive phenotypes observed in Per/Dec double mutant mice was seen in the regulation of locomotor activity onset (i.e., phase angle) under entrained conditions and after release into freerun (Bode et al., 2011a, 2011b). We did not measure rhythm entrainment in our cells (e.g., by ambient temperature cycles). However, because dexamethasonesynchronization represents a type 0 resetting paradigm, release into free-run can be simulated - and thus Bmalliluc phase differences between genotypes estimated-in vitro by comparing the time of the first peak of luminescence in rhythmic fibroblasts after synchronization, and it has been shown in cultured fibroblasts that after synchronization, the intrinsic phase relationship among the expressions of different clock genes are well preserved and comparable to the in vivo situation (Saini et al., 2012). WT ear fibroblasts peaked at $13.4 \pm 0.4 \mathrm{~h}$ after synchronization (Fig. $3 \mathrm{~A}$ ). In P1 cells-and similar to what was seen for period length (Fig. 2A, B) - phasing of the first peak was advanced $(11.0 \pm 0.6 \mathrm{~h})$, while in $D 2(17.4 \pm 0.1 \mathrm{~h}), P 1 D 2(17.3 \pm$ $0.6 \mathrm{~h})$, and $P 2 D 2(16.3 \pm 0.7 \mathrm{~h})$, cells peak phase was delayed relative to WT fibroblasts (Fig. 3A). In lungderived cells, the situation was similar with WT cells peaking at $11.2 \pm 1.2 \mathrm{~h}$ after synchronization, $P 1$ cells showing highest luciferase activity significantly earlier at $2.9 \pm 0.1 \mathrm{~h}$, and $D 2$ cells showing a delayed 
peak $(17.6 \pm 0.2 \mathrm{~h})$ compared with WT cells. Unlike what was seen in ear-derived cells, phasing in P2D2 lung cells $(9.5 \pm 1.0 \mathrm{~h})$ was not delayed relative to WT fibroblasts (Fig. 3B).

\section{Correlation between Period Length and Circadian Phase}

Previous data from animals and humans suggest a strong correlation between period length and phasing of behavioral and molecular rhythms (Brown et al., 2008; Liu et al., 2007; Meng et al., 2008; Oster et al., 2003; Ralph and Menaker, 1988; Zheng et al., 1999). In our study, we also observed some genotypes in which shortened period coincided with advanced phasing (e.g, P1 for both tissues; compare Figs. 2 and 3). To further analyze this relationship, we plotted peak time against period length for all preparations. When both tissues were pooled, no significant correlation between the 2 measures was found (data not shown). However, when we compared genotype effects separated for tissues, phasing did not correlate with period in ear-derived fibroblasts (Fig. 4A), whereas a strong correlation $(p<0.001)$ between the 2 parameters was seen for lung (Fig. 4B). In all cases, the extent of advance in phasing was much higher than that of period shortening, suggesting that period variations may contribute but are not sufficient to explain the observed phase advances.

\section{Interaction of PER and DEC Proteins in the Regulation of Dbp Transcription}

Our cell culture data suggested that Per1/2 and Dec2 have tissue type-independent synergistic roles in period regulation. So far, physical interaction of PER and DEC proteins has not been demonstrated, but it is known that they can both act on CLOCK/ BMAL1 activity at E-box promoters (Bode et al., 2011b; Honma et al., 2002; Jin et al., 1999; Rossner et al., 2008). Genetic and modeling experiments suggest that relieving E-box repression, for example by enhanced destabilization of PER proteins, shortens free-running period (Gallego et al., 2006; Maywood et al., 2011). We therefore hypothesized that synergistic de-repression of CLOCK/BMAL1 activity may underlie the interactive effect of Per1/2 and Dec2 deletions on period regulation. The opposite effect would be expected upon overexpression of PER and DEC proteins. To test this we measured luciferase activity in HEK $293 \mathrm{~T}$ cells transfected with a Dbp-luc reporter, Clock, and Bmal1 and various combinations of Per1,

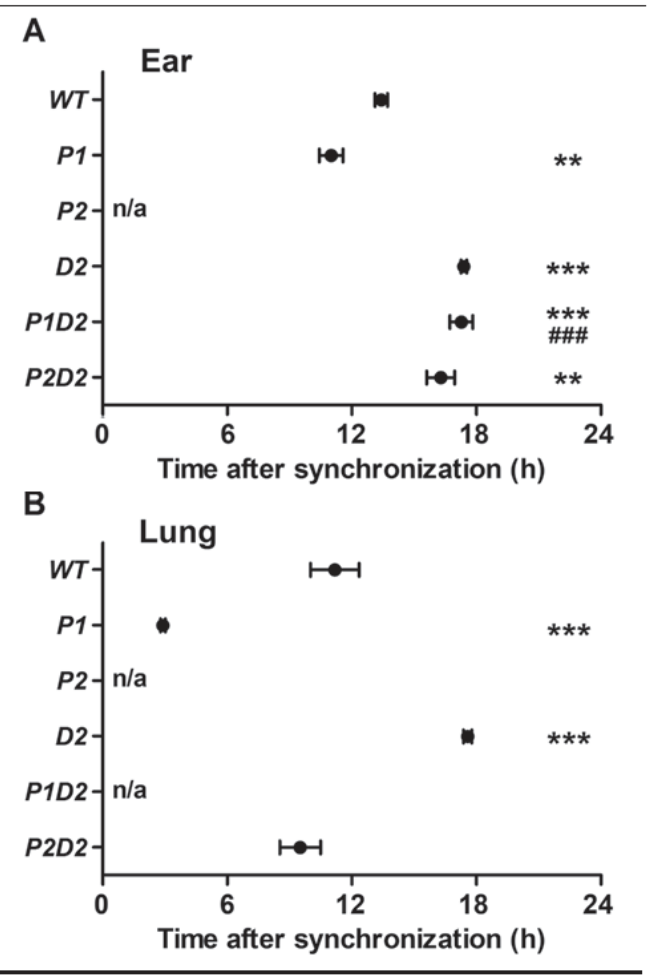

Figure 3. Bmallituc peak phase in ear- and lung-derived Perl Dec2 single and double mutant fibroblasts. Timing of first peaks of baseline-subtracted luminescence recordings after dexamethasone synchronization in ear-derived (A) and lungderived Bmall luc-transduced fibroblasts (B). All data are presented as means $\pm \operatorname{SEM}(n=6-7) ;{ }^{* *} p<0.01,{ }^{* * * * *} p<0.001,1$-way ANOVA with Bonferroni post-test vs. WT $\left(^{*}\right)$ or P1 $\left({ }^{(*)}\right)$ n/a $=$ no rhythm detectable.

Per2, and Dec2 expression plasmids. As expected, expression of Clock and Bmall resulted in a prominent increase in luciferase activity (Fig. 5A). Coexpression of Per1, Per2, or Dec2 alone led to a moderate inhibition of CLOCK/BMAL1 (although significance was not reached in a 1-way ANOVA). In contrast, cotransfection of Per1 or Per2 with Dec2 produced a significant repression of CLOCK/BMAL1 activity with luciferase activity levels comparable to control conditions. A second tissue type-independent finding from our fibroblast experiments was that P2D2 mutant cells had consistently longer periods than P1D2 mutants. Assuming that this effect was also mediated by differential regulation of CLOCK/BMAL1 transactivation and following the same argument as above, this would mean that PER1 and DEC1 together (i.e., 

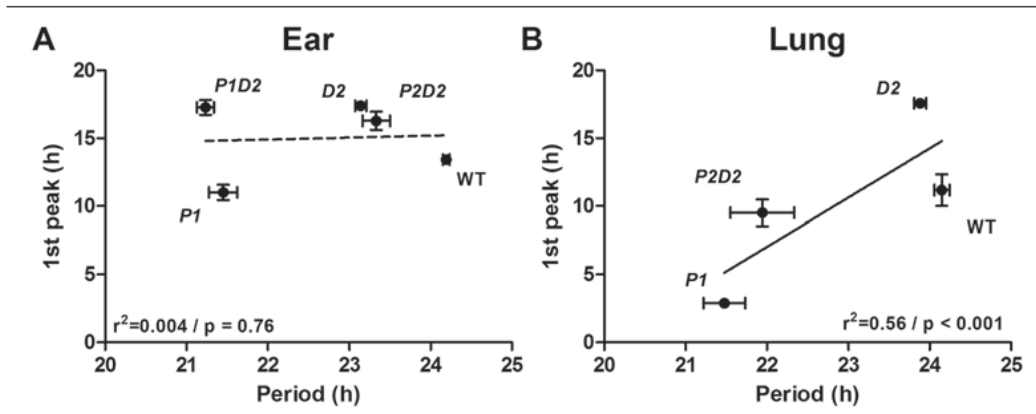

Figure 4. Correlation of phase vs. period length in ear- and lung-derived PerlDec2 single and double mutant fibroblasts. (A, B) Correlation of phase and period in ear-derived (A) and lung-derived synchronized fibroblasts (B) of wild-type (WT), Per1 (P1), Per2 (P2), Per1/Dec2 (P1D2), and Per2/Dec2 $(P 2 D 2)$ mutant mice. All data are presented as means \pm SEM $(n=6-7)$. Correlation lines and goodness of fit criteria are listed in each diagram. Solid lines depict significant degrees of correlation. clock regulation. This interaction is tissue-specific, affecting rhythmicity itself as well as rhythm sustainment, period length, and stability. In contrast, nonsynergistic interactions were seen in the regulation of cellular clock phase. Luciferase promoter assays suggest that Per/Dec interaction is mediated by interaction at the level of $E$-box regulation.
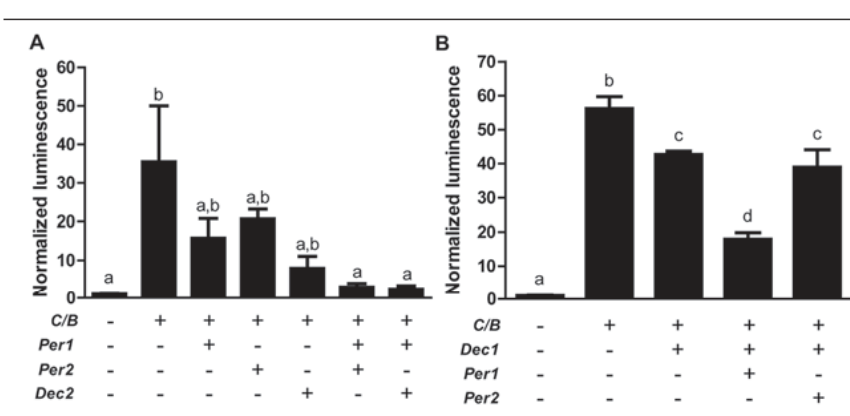

Figure 5. Interaction of Pers and Decs in the regulation of CLOCK/BMAL1-induced Dbp-luc activity. (A, B) Luciferase activity in HEK $293 \mathrm{~T}$ cells after transfection with different combinations of expression plasmids depicted below. Data are presented as means \pm SEM $(n=3)$; letters depict significantly different groups by 1-way ANOVA with Tukey posttest.

resembling the $\mathrm{P} 2 \mathrm{D} 2$ mutant situation) should have a stronger inhibitory effect on CLOCK/BMAL1 than PER2/DEC1 (resembling P1/D2). We tested this by cotransfecting Dec1 with Per1 or Per2 in HEK 293T cells as described above. While Dec1 alone had a moderate effect on CLOCK/BMAL1 activity, cotransfection with Per1, but not with Per2, caused further repression of Dbp-luc activity (Fig. 5B). Together, these data suggest that the interaction of Per1/2 and Dec2 in the regulation of the circadian TTL may - at least with respect to period-be mediated via E-box regulation.

\section{DISCUSSION}

Our data suggest a predominantly synergistic interaction between Per1, Per2, and Dec2 in cellular

\section{Synergistic but Tissue-Specific Interaction of Dec 2 and Per1/2}

It has previously been shown that the effects of clock gene mutations on circadian rhythmicity may differ between tissues (Cermakian et al,, 2001; DeBruyne et al., 2007b; Jakubcakova et al., 2007; Nakashima et al., 2008; Reick et al., 2001; Robles et al., 2010). Due to its unique intercellular coupling, the SCN pacemaker is particularly robust against genetic perturbation (Hogenesch and Herzog, 2011; Liu et al., 2007). This seems also true for Per/Dec mutations. When one compares the cellular data obtained from this study with previous reports on $\mathrm{Per} / \mathrm{Dec}$ mutant behavior and SCN clock gene expression (Bode et al,, 2011a, 2011b; Rossner et al., 2008; Zheng et al., 2001; Zheng et al., 1999), overall larger effects on cellular rhythmicity than on SCN-controlled locomotor behavior are observed (Table 1). For example, deletion of Dec2 had little effect on running-wheel activity rhythm period, phase, and stability, whereas period (ear), phase (ear and lung), and sustainment (lung) were affected in $D 2$ fibroblasts. The absence of communication between single oscillators in cell culture, however, may not be the only reason for the observed differences. Tissue-specific alterations in the clock gene machinery itself seem to also play a role as cells from ear or lung preparations react differently to genetic perturbation. For example, Bmal1:luc peak phase was delayed in $P 2 D 2$ ear fibroblasts, whereas 
Table 1. Comparison of behavioral and cellular phenotypes.

\begin{tabular}{|c|c|c|c|c|c|c|c|c|c|}
\hline \multirow[b]{2}{*}{ Mutation } & \multicolumn{3}{|c|}{ Behavior } & \multicolumn{3}{|c|}{ Ear Fibroblasts } & \multicolumn{3}{|c|}{ Lung Fibroblasts } \\
\hline & Period & Phase & Sustain ${ }^{a}$ & Period & Phase & Sustain ${ }^{\mathrm{b}}$ & Period & Phase & Sustain \\
\hline Per1 & $\downarrow$ & $\leftarrow$ & n.c. & $\downarrow$ & $\leftarrow$ & n.c. & $\downarrow$ & $\leftarrow$ & $\downarrow$ \\
\hline Per 2 & $\downarrow$ & $\leftarrow$ & $\downarrow$ & $\mathrm{x}$ & $x$ & $\downarrow c$ & $\mathrm{x}$ & $x$ & $\downarrow c$ \\
\hline Dec 2 & n.c. & n.c. & n.c. & $\downarrow$ & $\rightarrow$ & n.c. & n.c. & $\rightarrow$ & $\downarrow$ \\
\hline Per1/Dec2 & $\downarrow$ & $\leftarrow$ & n.c. & $\downarrow$ & $\rightarrow$ & $\uparrow$ & $\mathrm{x}$ & $\mathrm{x}$ & $\mathrm{x}$ \\
\hline Per2/Dec2 & $\downarrow$ & $\leftarrow$ & n.c. & $\downarrow$ & $\rightarrow$ & n.c. & $\downarrow$ & n.c. & $\downarrow$ \\
\hline
\end{tabular}

$\mathrm{x}=$ not determined; $\mathrm{n.c} .=$ no change; $\mathfrak{\imath}=$ reduced $/$ increased; $\leftrightarrow$ = advanced $/$ delayed.

a. Behavioral rhythmicity in constant darkness.

b. Bmal1: luc activity dampening time

b. Bmall: luc activity dampening time.
c. A lack of rhythmicity was interpreted as a sustain of 0 .

no change relative to WT was seen in lung-derived cells. In contrast, rhythm sustainment was unaltered-although rhythm power was strongly reduced-relative to WT cells in P2D2 ear fibroblasts whereas lung-derived cells showed accelerated dampening in their luminescence rhythm. Along this line, a mutation of Per1 has previously been shown to result in circadian arrhythmicity in dispersed SCN neurons and in tail tissue-derived fibroblasts (Liu et al., 2007), whereas rhythmicity was preserved in our setup (though with accelerated dampening and reduced rhythm power in lung). Together these data indicate a certain degree of variability in the composition of the clock gene machinery between tissues or, alternatively, the existence of tissue-specific modulators of clock function. A tissue-specific nature has previously been postulated for the role of Clock in circadian regulation (Kennaway et al., 2006). Interestingly, a mutation of Clock also differently affects Dec2 expression in different tissues (Noshiro et al., 2005).

\section{Dec2 Deletion Rescues Rhythmicity}

\section{in Per2 but Not in Per1 Mutant Cells}

A phenotypic rescue of clock rhythmicity after additional mutation of Dec2 in a Per2 mutant background was seen both at the behavioral level (Bode et al., 2011a) and in fibroblasts (Table 1), similar to what had previously been observed for circadian behavior and in vivo clock gene rhythms after deletion of Cry2 and Per2 (Oster et al., 2002). Together, these data suggest that Per 2 function in the circadian TTL is strongly modulated by other clock regulators and that under certain conditions, Per 2 may even be dispensable for circadian rhythm generation. This interpretation is further supported by recent studies that report normal rhythmicity and period regulation in Per2 ${ }^{\text {Idc }}$ mutant mice (Pendergast et al., 2010; $\mathrm{Xu}$ et al., 2007). Surprisingly, our data suggest that an additional mutation of Dec2 in a Per1 mutant background can stabilize cellular rhythm sustainment, while at the same time rhythm power is strongly reduced. It is generally believed that cellular oscillators in culture do not communicate with each other (Liu et al., 2007). Therefore, rhythm dampening in cell culture is mainly based on 2 factors: individual differences in endogenous cellular period lengths and rhythm destabilization at the molecular level. Imaging studies in WT fibroblast cultures show that cellular clocks are self-sustained and single cells robustly cycle over extended periods of time while overall phase coherence is rapidly lost (Leise et al., 2012; Nagoshi et al., 2004). Our data support the hypothesis that individual period variation may contribute to rapid dampening in Per1 mutant cells and that-specifically in ear-derived fibroblasts-this effect is compensated after additional mutation of Dec2. The finding that in both P1D2 and P2D2 double mutant cells rhythm sustainment does not correlate with increased rhythm power indicates a rather high variability in day-to-day period regulation, rendering the remaining rhythm highly unreliable. Per1 mutant mice show a high variability in locomotor activity, rhythm period, and length regulation under free-running conditions (Zheng et al,, 2001), and this effect is preserved in P1D2 double mutant animals (Bode et al., 2011b) and cells (this article), suggesting that the absence of Per genes may confer intrinsic instability to the clock without principally affecting rhythm sustainment per se. However, lacking singlecell recordings, we cannot distinguish between interand intracellular effects in our setup.

The observation that rhythm sustainment in earderived P1D2 mutant cells seems improvedalthough with reduced power-when compared 
with WT cells appears surprising but may emphasize 2 important aspects. First, circadian clocks have not evolved under free-running conditions. Although for physiological entrainment it is important that clocks are capable of predicting time under zeitgeber free conditions and as a counterbalance against external noise, for most species optimization of entrainment under natural zeitgeber cycles might have been evolutionarily much more relevant (Hut and Beersma, 2011). Second, cell culture conditions only poorly recapitulate the native environment to which a cell is exposed inside the body. Thus, interactions between the altered genetic machinery of a mutant cell and components of the culture medium may critically interfere with the regulation and stability of cellular circadian rhythms. Some components of the clock gene machinery have been shown to interact with external signaling pathways. For example, CRY proteins regulate glucocorticoid receptor signaling and cellular energy metabolism in murine fibroblasts (Lamia et al., 2011). Such implications should be taken into consideration when interpreting circadian phenotypes under in vitro conditions.

\section{Nonsynergistic Interaction of Per1 and Dec2 in the Regulation of Cellular Clock Phase}

Our analyses of clock phase reveal 2 major findings. First, loss of Per1 has an opposite effect on Bmal1-luc phase regulation than loss of Dec2. In earderived P1D2 double mutant cells, phase regulation resembles that of $D 2$ mutants, whereas in lungderived cells, loss of both genes results in arrhythmicity. Second, peak phase relations between different genotypes are not always correlated to period length. Theoretical considerations suggest that entrainment phase of an oscillatory system to an external zeitgeber is influenced by internal period (Roenneberg et al., 2008). Experimental data from human and animal studies support these postulations (Brown et al., 2008; Ralph and Menaker, 1988; Toh et al., 2001; Xu et al., 2005; Zheng et al., 1999). The extent of this effect, however, is highly dependent on zeitgeber strength. Activity rhythms in rodents, for example, can be stably entrained by extremely low light intensities (Foster et al., 2007), and standard laboratory light regimens are usually far beyond this entrainment threshold, thus minimizing the observed effects of internal period on phase adjustment. In cell culture conditions, the determination of phase will further depend on the selected readout. In this project we used luciferase activity as a real-time reporter of Bmal1 promoter activity. However, with deletion of TTL components in clock gene mutant cells, the normal phase relationship between different TTL transcripts may be lost. Thus, phase relationships between genotypes may be different if another readout (e.g., another clock gene) is chosen. Of note, such shifts in clock gene mRNA profiles were also observed in Per/ Dec mutant SCN (Bode et al., 2011a, 2011b). Third, genetic mutations may differentially affect resetting pathways and period regulation (Jakubcakova et al., 2007). The fact that in Per/Dec mutant fibroblasts phase differences are much more pronounced than those observed in free-running period lengths suggests that this may represent a major factor in this context. This assumption is supported by the fact that both Per genes have been implicated in resetting of the TTL in both the SCN and the periphery (Dibner et al., 2010).

\section{Per1/2 and Dec2 Interact at the Level of E-Box Regulation}

Our reporter gene assays show that Per1/2 and $D e c 2$ interact in regulating CLOCK/BMAL1 activity on the Dbp promoter. Such mode of interaction has been suggested before for mammals (Rossner et al., 2008) and for the Per/Dec orthologs period and clockwork orange of the Drosophila clock (Kadener et al., 2007). In vitro experiments on nonsynchronized cells, however, only poorly reflect the dynamics of an oscillatory system, and it has indeed been suggested that Dec function in E-box regulation may depend on clock phase (Rossner et al., 2008). A direct physical interaction of PER and DEC proteins has so far not been shown, but even though our transcription assay data indicate that E-box effects are sufficient to explain the period effects observed in Per1/2-Dec2 mutant cells, other mechanisms may play a role or become even more important for the regulation of clock phasc and stability or for cxplaining tissuc specific effects of Per/Dec mutations.

\section{CONCLUSION}

The analysis of oscillatory behavior after combinatorial deletion of different genetic components of the biological clock, in particular in cells, may help us to better understand the complex interactions of the numerous components of the circadian clockwork (for a successful example in plants, see Locke et al., 
2005). In mammals this approach is hampered by the fact that multiple mutations frequently result in arrhythmicity, which then prevents further measurements (Cho et al., 2012; DeBruyne et al., 2007a; Oster et al., 2002). We show that in cells, Per/Dec2 interaction is either synergistic (period and rhythm power) or nonsynergistic (phase) and highly tissue-specific. Given that in the SCN, Dec1 and Dec2 have partly redundant function (Honma et al., 2002; Rossner et al., 2008), it would be interesting to extend these studies to DecI mutant cells. Many clock components specifically regulate biological processes outside circadian timekeeping (Bradshaw and Holzapfel, 2010; Franken et al., 2007; Rosenwasser, 2010; Sidman et al., 1962). Therefore, describing the modes of clock gene interactions in different tissues may be particularly useful to identify pharmacological targets for the tissue-specific intervention into clock-controlled as well as noncircadian physiological systems.

\section{ACKNOWLEDGMENTS}

We would like to thank Jana Husse for critical comments on the manuscript. This project was funded by the German Research Foundation (DFG; OS 353/2-1 \& KГO 241). H.O. is an Emmy Noether fellow of the DFG and a Lichtenberg fellow of the Volkswagen Foundation. A.H.T. is supported by a GGNB Excellence Fellowship from the University of Göttingen. M.J.R. is supported by the DFG Research Center Molecular Physiology of the Brain (CMPB).

\section{CONFLICT OF INTEREST STATEMENT}

The author(s) declared no potential conflicts of interest with respect to the research, authorship, and/or publication of this article.

\section{NOTE}

Supplementary online material is available on the journal's website at http://jbr.sagepub.com/supplemental.

\section{REFERENCES}

Aton SJ, Colwell CS, Harmar AJ, Waschek J, and Herzog ED (2005) Vasoactive intestinal polypeptide mediates circadian rhythmicity and synchrony in mammalian clock neurons. Nat Neurosci 8:476-483.
Bode B, Rossner M], and Oster H (2011a) Advanced lightentrained activity onsets and restored free-running suprachiasmatic nucleus circadian rhythms in per2/dec mutant mice. Chronobiol Int 28:737-750.

Bode B, Shahmoradi A, Rossner MJ, and Oster H (2011b) Genetic interaction of per1 and dec1/ 2 in the regulation of circadian locomotor activity. J Biol Rhythms 26:530540 .

Bradshaw WE and Holzapfel CM (2010) Circadian clock genes, ovarian development and diapause. BMC Biol $8: 115$.

Brown SA, Kowalska E, and Dallmann R (2012) (Re)inventing the circadian feedback loop. Dev Cell 22:477-487.

Brown SA, Kunz D, Dumas A, Westermark PO, Vanselow $\mathrm{K}$, Tilmann-Wahnschaffe A, Herzel $\mathrm{H}$, and Kramer A (2008) Molecular insights into human daily behavior. Proc Natl Acad Sci U S A 105:1602-1607.

Bunger MK, Wilsbacher LD, Moran SM, Clendenin C, Radcliffe LA, Hogenesch JB, Simon MC, Takahashi JS, and Bradfield CA (2000) Mop3 is an essential component of the master circadian pacemaker in mammals. Cell 103:1009-1017.

Cermakian N, Monaco L, Pando MP, Dierich A, and Sassone-Corsi P (2001) Altered behavioral rhythms and clock gene expression in mice with a targeted mutation in the Period1 gene. EMBO J 20:3967-3974.

Cho H, Zhao X, Hatori M, Yu RT, Barish GD, Lam MT, Chong LW, Ditacchio L, Atkins AR, Glass CK, et al. (2012) Regulation of circadian behaviour and metabolism by REV-ERB-alpha and REV-ERB-beta. Nature. 485:123-127.

DeBruyne JP, Weaver DR, and Reppert SM (2007a) CLOCK and NPAS2 have overlapping roles in the suprachiasmatic circadian clock. Nat Neurosci 10:543-545.

DeBruyne JP, Weaver DR, and Reppert SM (2007b) Peripheral circadian oscillators require CLOCK. Curr Biol 17:R538-R539.

Dibner C, Schibler U, and Albrecht U (2010) The mammalian circadian timing system: organization and coordination of central and peripheral clocks. Annu Rev Physiol 72:517-549.

Foster RG, Hankins MW, and Peirson SN (2007) Light, photoreceptors, and circadian clocks. Methods Mol Biol 362:3-28.

Franken P, Thomason R, Heller HC, and O'Hara BF (2007) A non-circadian role for clock-genes in sleep homeostasis: a strain comparison. BMC Neurosci 8:87.

Gallego M, Eide EJ, Woolf MF, Virshup DM, and Forger DB (2006) An opposite role for tau in circadian rhythms revealed by mathematical modeling. Proc Natl Acad Sci U S A 103:10618-10623.

Gekakis N, Staknis D, Nguyen HB, Davis FC, Wilsbacher LD, King DP, Takahashi JS, and Weitz CJ (1998) Role of the CLOCK protein in the mammalian circadian mechanism. Science 280:1564-1569.

Griffin EA, Jr., Staknis D, and Weitz CJ (1999) Lightindependent role of CRY1 and CRY2 in the mammalian circadian clock. Science 286:768-771.

Hamaguchi H, Fujimoto K, Kawamoto T, Noshiro M, Maemura K, Takeda N, Nagai R, Furukawa M, Honma S, Honma K, et al. (2004) Expression of the gene for Dec2, 
a basic helix-loop-helix transcription factor, is regulated by a molecular clock system. Biochem J 382:43-50.

Hogenesch JB and Herzog ED (2011) Intracellular and intercellular processes determine robustness of the circadian clock. FEBS Lett 585:1427-1434.

Honma S, Kawamoto T, Takagi Y, Fujimoto K, Sato F, Noshiro M, Kato Y, and Honma K (2002) Dec1 and Dec2 are regulators of the mammalian molecular clock. Nature 419:841-844.

Hut RA and Beersma DG (2011) Evolution of time-keeping mechanisms: early emergence and adaptation to photoperiod. Philos Trans R Soc Lond B Biol Sci 366:21412154.

Jakubcakova V, Oster H, Tamanini F, Cadenas C, Leitges M, van der Horst GT, and Eichele G (2007) Light entrainment of the mammalian circadian clock by a PRKCAdependent posttranslational mechanism. Neuron 54:831-843.

Jin $X$, Shearman LP, Weaver DR, Zylka MJ, de Vries GJ, and Reppert SM (1999) A molecular mechanism regulating rhythmic output from the suprachiasmatic circadian clock. Cell 96:57-68.

Kadener S, Stoleru D, McDonald M, Nawathean P, and Rosbash M (2007) Clockwork Orange is a transcriptional repressor and a new Drosophila circadian pacemaker component. Genes Dev 21:1675-1686.

Kennaway DJ, Owens JA, Voultsios A, and Varcoe TJ (2006) Functional central rhythmicity and light entrainment, but not liver and muscle rhythmicity, are Clock independent. Am J Physiol Regul Integr Comp Physiol 291:R1172-R1180.

Kume K, Zylka MJ, Sriram S, Shearman LP, Weaver DR, Jin X, Maywood ES, Hastings MH, and Reppert SM (1999) mCRY1 and mCRY2 are essential components of the negative limb of the circadian clock feedback loop. Cell 98:193-205.

Lamia KA, Papp SI, Yu RT, Barish GD, Uhlenhaut NH, Jonker JW, Downes M, and Evans RM (2011) Cryptochromes mediate rhythmic repression of the glucocorticoid receptor. Nature 480:552-556.

Lee C, Etchegaray JP, Cagampang FR, Loudon AS, and Reppert SM (2001) Posttranslational mechanisms regulate the mammalian circadian clock. Cell 107:855-867.

Leise TL, Wang CW, Gitis PJ, and Welsh DK (2012) Persistent cell-autonomous circadian oscillations in fibroblasts revealed by six-week single-cell imaging of PER2::LUC bioluminescence. PLoS One 7:e33334.

Liu AC, Welsh DK, Ko CH, Tran HG, Zhang EE, Priest AA, Buhr ED, Singer O, Meeker K, Verma IM, et al. (2007) Intercellular coupling confers robustness against mutations in the SCN circadian clock network. Cell 129: 605-616.

Liu C and Reppert SM (2000) GABA synchronizes clock cells within the suprachiasmatic circadian clock. Neuron $25: 123-128$.

Locke JC, Millar AJ, and Turner MS (2005) Modelling genetic networks with noisy and varied experimental data: the circadian clock in Arabidopsis thaliana. J Theor Biol 234:383-393.

Maywood ES, Chesham JE, Meng QI, Nolan PM, Loudon AS, and Hastings $\mathrm{MH}$ (2011) Tuning the period of the mammalian circadian clock: additive and independent effects of CK1epsilonTau and Fbxl3Afh mutations on mouse circadian behavior and molecular pacemaking. J Neurosci 31:1539-1544.

Maywood ES, Reddy AB, Wong GK, O'Neill JS, O'Brien JA, McMahon DG, Harmar AJ, Okamura $\mathrm{H}$, and Hastings MH (2006) Synchronization and maintenance of timekeeping in suprachiasmatic circadian clock cells by neuropeptidergic signaling. Curr Biol 16:599-605.

Meng QJ, Logunova L, Maywood ES, Gallego M, Lebiecki J, Brown TM, Sladek M, Semikhodskii AS, Glossop NR, Piggins HD, et al. (2008) Setting clock speed in mammals: the CK1 epsilon tau mutation in mice accelerates circadian pacemakers by selectively destabilizing PERIOD proteins. Neuron 58:78-88.

Nagoshi E, Saini C, Bauer C, Laroche T, Naef F, and Schibler U (2004) Circadian gene expression in individual fibroblasts: cell-autonomous and self-sustained oscillators pass time to daughter cells. Cell 119:693-705.

Nakashima A, Kawamoto T, Honda KK, Ueshima T, Noshiro M, Iwata T, Fujimoto K, Kubo H, Honma S, Yorioka N, et al. (2008) DEC1 modulates the circadian phase of clock gene expression. Mol Cell Biol 28:40804092.

Noshiro M, Furukawa M, Honma S, Kawamoto T, Hamada T, Honma K, and Kato Y (2005) Tissue-specific disruption of rhythmic expression of Dec1 and Dec2 in clock mutant mice. J Biol Rhythms 20:404-418.

Oster H (2006) The genetic basis of circadian behavior. Genes Brain Behav 5 Suppl 2:73-79.

Oster H, Baeriswyl S, Van Der Horst GT and Albrecht U (2003) Loss of circadian rhythmicity in aging mPer1-/mCry2-/- mutant mice. Genes Dev 17:1366-1379.

Oster H, Yasui A, van der Horst GT, and Albrecht U (2002) Disruption of mCry2 restores circadian rhythmicity in mPer2 mutant mice. Genes Dev 16:2633-2638.

Pando MP, Morse D, Cermakian N, and Sassone-Corsi P (2002) Phenotypic rescue of a peripheral clock genetic defect via SCN hierarchical dominance. Cell 110:107-117.

Pendergast JS, Friday RC, and Yamazaki S (2010) Distinct functions of Period 2 and Period 3 in the mouse circadian system revealed by in vitro analysis. PLoS One 5:e8552.

Preitner N, Damiola F, Lopez-Molina L, Zakany J, Duboule D, Albrecht U, and Schibler U (2002) The orphan nuclear receptor REV-ERBalpha controls circadian transcription within the positive limb of the mammalian circadian oscillator. Cell 110:251-260.

Ralph MR and Menaker M (1988) A mutation of the circadian system in golden hamsters. Science 241:1225-1227.

Reick M, Garcia JA, Dudley C, and McKnight SL (2001) NPAS2: an analog of clock operative in the mammalian forebrain. Science 293:506-509.

Robles MS, Boyault C, Knutti D, Padmanabhan K, and Weitz CJ (2010) Identification of RACK1 and protein kinase $\mathrm{C}$ alpha as integral components of the mammalian circadian clock. Science 327:463-466.

Roenneberg T, Chua EJ, Bernardo R, and Mendoza E (2008) Modelling biological rhythms. Curr Biol 18:R826-R835.

Rosenwasser AM (2010) Circadian clock genes: non-circadian roles in sleep, addiction, and psychiatric disorders? Neurosci Biobehav Rev 34:1249-1255. 
Rossner MJ, Oster $\mathrm{H}$, Wichert SP, Reinecke L, Wehr MC, Reinecke J, Eichele G, Taneja R, and Nave KA (2008) Disturbed clockwork resetting in Sharp-1 and Sharp-2 single and double mutant mice. PLoS One 3:e2762.

Saini C, Morf J, Stratmann M, Gos P, and Schibler U (2012) Simulated body temperature rhythms reveal the phaseshifting behavior and plasticity of mammalian circadian oscillators. Genes Dev 26:567-580.

Sidman RL, Lane PW, and Dickie MM (1962) Staggerer, a new mutation in the mouse affecting the cerebellum. Science 137:610-612.

Sun ZS, Albrecht U, Zhuchenko O, Bailey J, Eichele G, and Lee CC (1997) RIGUI, a putative mammalian ortholog of the Drosophila period gene. Cell 90:1003-1011.

Takahashi JS, Hong HK, Ko CH, and McDearmon EL (2008) The genetics of mammalian circadian order and disorder: implications for physiology and disease. Nat Rev Genet 9:764-775.

Toh KL, Jones CR, He Y, Eide EJ, Hinz WA, Virshup DM, Ptacek LJ, and Fu YH (2001) An hPer2 phosphorylation site mutation in familial advanced sleep phase syndrome. Science 291:1040-1043.

Xu Y, Padiath QS, Shapiro RE, Jones CR, Wu SC, Saigoh N, Saigoh K, Ptacek LI, and Fu YH (2005) Functional consequences of a CKIdelta mutation causing familial advanced sleep phase syndrome. Nature 434:640-644.
Xu Y, Toh KL, Jones CR, Shin JY, Fu YH, and Ptacek LJ (2007) Modeling of a human circadian mutation yields insights into clock regulation by PER2. Cell 128:59-70.

Yamaguchi S, Isejima H, Matsuo T, Okura R, Yagita K, Kobayashi M, and Okamura H (2003) Synchronization of cellular clocks in the suprachiasmatic nucleus. Science 302:1408-1412.

Yoo SH, Yamazaki S, Lowrey PL, Shimomura K, Ko $\mathrm{CH}$, Buhr ED, Siepka SM, Hong HK, Oh WJ, Yoo OJ, et al. (2004) PERIOD2::LUCIFERASE real-time reporting of circadian dynamics reveals persistent circadian oscillations in mouse peripheral tissues. Proc Natl Acad Sci U S A 101:5339-5346.

Yoshitane $\mathrm{H}$, Takao T, Satomi $\mathrm{Y}, \mathrm{Du} \mathrm{NH}$, Okano $\mathrm{T}$, and Fukada Y (2009) Roles of CLOCK phosphorylation in suppression of E-box-dependent transcription. Mol Cell Biol 29:3675-3686.

Zheng B, Albrecht U, Kaasik K, Sage M, Lu W, Vaishnav S, Li Q, Sun ZS, Eichele G, Bradley A, et al. (2001) Nonredundant roles of the mPer1 and mPer2 genes in the mammalian circadian clock. Cell 105:683-694.

Zheng B, Larkin DW, Albrecht U, Sun ZS, Sage M, Eichele G, Lee CC, and Bradley A (1999) The mPer2 gene encodes a functional component of the mammalian circadian clock. Nature 400:169-173. 